


\section{Propositions}

1. Levels of iron and vitamin D should be monitored in PAH patients. (this thesis)

2. A diet rich in protein and components with anti-inflammatory properties provides a promising strategy to reduce adverse effects in cardiac and skeletal muscle tissue in PAH patients.

(this thesis)

3. Gender bias is a persistent cause of knowledge gaps regarding diseases that are more common in women than in men.

4. Nutritional scientists should actively combat misguiding information about nutrition and health in the public discourse.

5. Competitive grant writing by scientists comes at high societal costs.

6. Welcoming a child into your life helps to find work-life balance.

Propositions belonging to the thesis, entitled

Nutrition as potential treatment strategy in pulmonary arterial hypertension

Paulien Vinke

Wageningen, 9 December 2021 

Nutrition as potential treatment strategy in pulmonary arterial hypertension

Paulien Vinke 


\section{Thesis committee}

\section{Promotors}

Prof. Dr Renger F. Witkamp

Professor of Nutritional Biology

Wageningen University \& Research

\section{Co-promotors}

Dr Klaske van Norren

Associate Professor, Human Nutrition \& Health

Wageningen University \& Research

Dr Karin A. Boomars

Pulmonary Physician

Erasmus MC University Medical Center Rotterdam

\section{Other members}

Prof. Dr E Kampman, Wageningen University \& Research

Prof. Dr A.H.E.M. Maas, Radboud University Nijmegen

Prof. Dr J. Garssen, University Utrecht

Prof. Dr M. Post, University Utrecht

This research was conducted under the auspices of the Graduate School VLAG (Advanced studies in Food Technology, Agrobiotechnology, Nutrition and Health Sciences). 


\title{
Nutrition as potential treatment strategy in pulmonary arterial hypertension
}

\author{
Paulien Vinke
}

Thesis

submitted in fulfilment of the requirements for the degree of doctor at Wageningen University

by the authority of the Academic Board,

in the presence of the

Thesis Committee appointed by the Academic Board

to be defended in public

on Thursday 9 December 2021

at 11 a.m. in the Aula. 
Paulien Vinke

Nutrition as potential treatment strategy in pulmonary arterial hypertension 173 pages

PhD thesis, Wageningen University, Wageningen, the Netherlands (2021)

With references, with summaries in English and Dutch

ISBN 978-94-6447-002-4

DOI: https://doi.org/10.18174/555571 


\section{Table of Contents}

1 Introduction: Prevalence of micronutrient deficiencies and potential of nutritional interventions in pulmonary arterial hypertension

2 Increasing quality of life in pulmonary arterial hypertension: is there a role for nutrition?

3 Prevalence of micronutrient deficiencies and relation with clinical and patientrelated outcomes in pulmonary hypertension type I and IV 29

4 The use of proton pump inhibitors may increase symptoms of muscle function loss in patients with chronic illnesses 55

5 Anti-inflammatory nutrition with high protein attenuates cardiac and skeletal muscle alterations in a pulmonary arterial hypertension model .77

6 Synthesis, discussion, and conclusion 99

References 112

Appendix I 147

Appendix II. 151

Appendix III 153

Appendix IV 154

Appendix V 155

Appendix VI 156

English summary..... 159

Nederlandse samenvatting.. 162

Acknowlegdements. 166

Curriculum vitae 170

Selected publications 171

Overview of completed training activities 173 


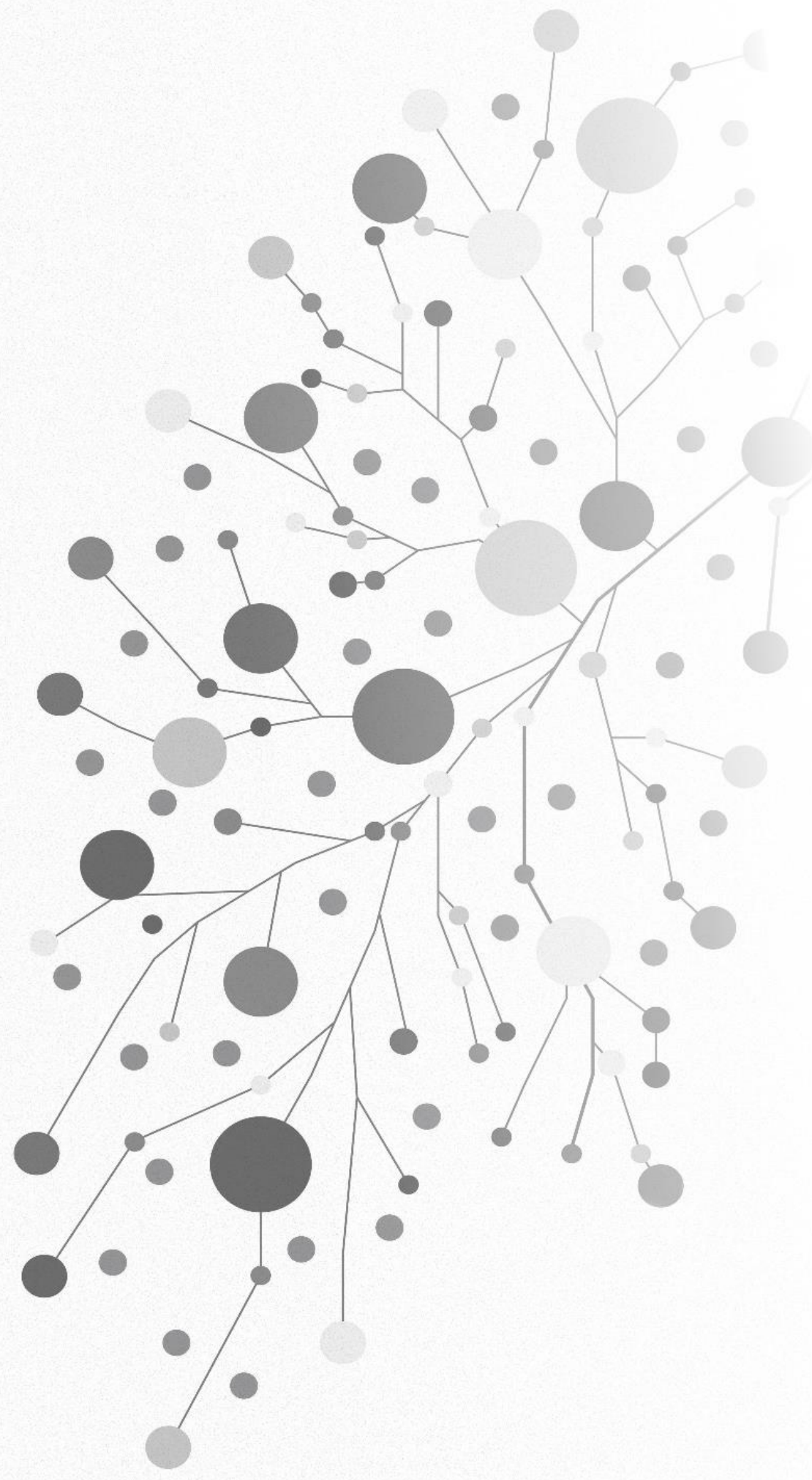


1 Introduction: Prevalence of micronutrient deficiencies and potential of nutritional interventions in pulmonary arterial hypertension 


\subsection{Pulmonary arterial hypertension}

Pulmonary hypertension (PH) refers to a heterogeneous group of diseases characterized by vasoconstriction and structural remodelling of the pulmonary arteries. Increased vascular resistance and elevated pulmonary arterial pressures contribute to right ventricular hypertrophy and eventually lead to heart failure and premature death (Hoeper et al., 2013; Simonneau et al., 2019). Symptoms of PH are progressive and include serious exercise intolerance, reducing the quality of life of patients. Important pathophysiological mechanisms contributing to exercise intolerance and fatigue in $\mathrm{PH}$ include right ventricular dysfunction and inflammation, which among other things result in skeletal muscle alterations and dysfunctional energy metabolism (Tran et al., 2018; Vinke et al., 2018). Currently, PH is divided into 5 subgroups according to the classification of the World Health Organisation (WHO), European Respiratory Society (ERS) and European Society of Cardiology (ESC). The first subgroup is pulmonary arterial hypertension (PAH), WHO type $2 \mathrm{PH}$ is related to left heart disease, type $3 \mathrm{PH}$ is due to lung disease and hypoxia, type 4 is due to chronic thrombo-embolic pulmonary hypertension (CTEPH) or other pulmonary artery obstructions, while type $5 \mathrm{PH}$ is due to multifactorial mechanisms (Galiè, Humbert, et al., 2015). PAH consists of different subgroups based upon different underlying disorders, such as PAH due to genetic mutations, congenital heart disease, connective tissue disease, liver disease, HIV, and, when the etiology is unknown: idiopathic PAH. In both PAH and CTEPH the disease originates in the pulmonary arteries. Those patients are typically treated with $\mathrm{PH}$-specific therapies in expert centers, whereas other $\mathrm{PH}$ patients receive different treatment.

Prognosis of both PAH and CTEPH is poor, with 3- and 5-year survival rates for PAH of $67 \%$ and $57-59 \%$, respectively (Benza, Miller, et al., 2012; Galiè, Humbert, et al., 2015; Rådegran et al., 2016), and 5-year survival rates of 53-69\% for inoperable CTEPH (Boucly et al., 2017; Quadery et al., 2018; Rådegran et al., 2016). The prognosis of CTEPH patients with central laesions, who are eligible for and who underwent a pulmonary endarterectomy is improved with 5-years survival rates of around $85 \%$ (Sandqvist et al., 2021). Based on risk stratification criteria for the determination of prognosis of PAH patients (1-year mortality), the following predictors of poor prognosis were determined by the ESC and the ERS: advanced 
functional class, poor exercise capacity and right ventricular dysfunction (Galiè, Humbert, et al., 2015). The stage of heart failure (functional class) is diagnosed according to the classification of the New York Heart Association (NYHA) with class I meaning that a patient experiences no symptoms or limitation in ordinary physical activity, class II having mild symptoms, class III meaning marked symptoms even at less-than-ordinary activity and class IV meaning that the patient is severely limited with symptoms even at rest (The Criteria Committee of the New York Heart Association, 1994).

\subsection{Nutritional intervention in chronic diseases}

Nutrition is increasingly being recognized as an important factor contributing to quality of life during disease, and patients are actively seeking advice on such matters. For several chronic diseases such as cancer, diabetes, coronary heart disease (CHD), chronic obstructive pulmonary disease (COPD) and others, more and more evidence is generated showing that lifestyle interventions in general, and dietary interventions specifically, can have major impact on the quality of life and lifespan of patients (Arends et al., 2017; Schols et al., 2014; von Haehling et al., 2017). For some chronic diseases such as cancer, $\mathrm{CHD}$ and COPD nutritional guidelines have been developed (Anker et al., 2006, 2009; Arends et al., 2017). However, nutritionrelated guidelines for PAH treatment are scarce and there is limited knowledge on this topic. Moreover, the pathophysiological background of each type of $\mathrm{PH}$ may impact nutritional status differently.

\subsection{Nutritional interventions for PAH patients: where do we stand?}

To get a better understanding of the current situation around nutritional interventions for $\mathrm{PAH}$ patients, I interviewed specialized PAH health care professionals, rehabilitation teams and a representative of the Dutch patient association in the Netherlands. The results of these interviews showed that an increased interest and awareness of the potential role of nutrition and physical activity are present. However, there are also several relevant knowledge gaps on this topic.

The interviews showed that cardiologists and pulmonologists do not assess nutritional status, nutrition-related problems, or loss of muscle mass in PAH patients as part of the routine practice for PAH patients. Typical micronutrient 
deficiencies, such as iron, vitamin B12 and folic acid do receive some attention. The measurement and discussion of exercise tolerance are more or less standard practice, but not standardized into diagnostic tools. However, signs of loss of muscle function, fatigue and problems performing daily activities, are commonly reported by the interviewed cardiologists and pulmonologists. Although the symptoms are also likely to result from the disease itself, nutrient deficiencies may play a role here.

Specialized PAH nurses indicate that they receive many questions from $\mathrm{PAH}$ patients about the use of specific food supplements, especially from younger patients. Currently, there is no evidence-based knowledge available to be able to answer such questions. For example, there is an awareness, based on studies in other populations, that protein supplementation might increase effectiveness of exercise interventions. However, due to lack of scientific evidence in this patient group, it is impossible provide any recommendations.

PAH experts indicate that PAH patients often do not look undernourished till the point that they develop severe heart failure. Factors that may contribute to weight loss and malnourishment could be side effects of medication such as nausea, fatigue, edema, and diarrhea. On the other hand, an increase in weight in PAH patients might be observed due to fluid retention or due to exercise intolerance and inactivity without adapting eating habits.

To summarize, there is an increasing awareness of the potential role of nutrition in $\mathrm{PAH}$, but assessment of nutritional status or the analysis of micronutrient concentrations are currently not standard practice. Also, nutritional intervention is not part of regular treatment, due to a lack of evidence-based recommendations for PAH patients.

\subsection{Objectives of this thesis}

As has been outlined above, several knowledge gaps exist on the prevalence of micronutrient deficiencies, their relevance, and the potential of nutritional interventions in patients with pulmonary hypertension.

The main objectives of this thesis are thus to gain more knowledge on the prevalence of micronutrient deficiencies of patients with pulmonary hypertension and to explore possible nutritional interventions to reduce fatigue and improve exercise 
tolerance and quality of life in PAH patients. These objectives are addressed by 1) literature research and hypothesis generation, 2) measurement of micronutrient status in PAH and CTEPH patients, 3) exploration of the relation between levels of micronutrients and clinical and patient-related outcomes and 4) a study on the effect of nutritional intervention on muscle function in a mouse model of pulmonary hypertension.

These objectives lead to the following research questions:

- What is known about the nutritional status of patients with pulmonary hypertension and how does the pathophysiology of $\mathrm{PAH}$ impact the relation between nutritional status, exercise intolerance and fatigue?

- What is the prevalence of micronutrient deficiencies in patients with pulmonary hypertension and can deficiencies in certain micronutrients be related to clinical and patient-related outcomes?

- Can the use of certain medications, such as proton pump inhibitors, contribute to a reduction in muscle mass and function in chronically ill patients?

- Can nutritional intervention reduce cardiac and skeletal muscle changes in PAH?

\subsection{Outline of the thesis}

This thesis consists of 6 chapters (Figure 1.1). Chapters 2 to 5 are conceptualized and written as independent scientific papers and can thus be read separately. In chapter 2, an overview of current knowledge on nutritional status in patients with pulmonary hypertension is given. Also, the relationships between the pathophysiology of $\mathrm{PAH}$ with nutritional status, exercise intolerance and fatigue are explored (research question 1). The review shows that there is hardly any knowledge on the prevalence of micronutrient deficiencies in PAH patients, although typical symptoms of the disease can lead to an impaired nutritional status and so to lower quality of life. In Chapter 3 the results of a study measuring the prevalence of micronutrient deficiencies in both newly diagnosed and treated patients with PAH and chronic thrombo-embolic pulmonary hypertension (CTEPH) are described. Deficiencies in both iron and vitamin D are highly prevalent in both PAH and CTEPH patients at diagnosis as well as after 1,5 years of treatment. Iron plasma 
levels correlated with 6-minute walk distance. Low iron levels seem to be related to inflammation via hepcidin (research question 2). In chapter 2, medication use is suggested as a potential factor influencing nutritional status in PAH patients. Therefore, in chapter 4, the hypothesis that the use of proton pump inhibitors (which are also highly used by $\mathrm{PH}$ patients) contributes to a reduction in muscle mass and function in chronically ill patients (research question 3) is elaborated on. As it becomes clear from chapters 2 and 3, inflammation is a recurring factor in the pathophysiology of PAH that is not only related to disease outcome, but also to nutritional factors. To further address this, a study exploring the effects of antiinflammatory components in the diet on cardiac and skeletal muscle in a mouse model is described in Chapter 5. Results presented in this chapter show that a multitargeted nutritional intervention with extra protein, leucine, and the antiinflammatory components of fish oil and oligosaccharides can prevent pathological changes in both cardiac and skeletal muscle in this mouse model of PAH (research question 4). Chapter 6 contains a general synthesis of the thesis, in which the methodologies and results are discussed together. Furthermore, suggestions for future lines of research are provided. 


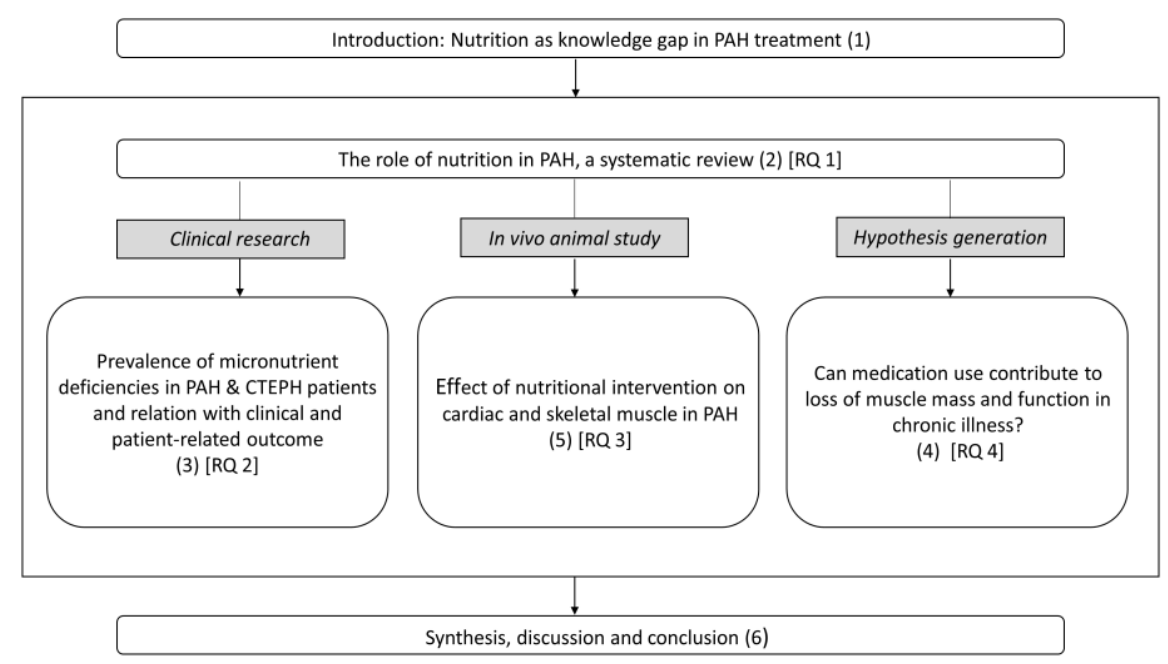

Figure 1.1: Outline of the thesis: Numbers in brackets refer to chapters, numbers in square brackets refer to research questions.

\section{Box 1. Pulmonary arterial hypertension: facts \& numbers}

Prevalence of PAH: approx. 25 subjects per million population (Maron et al., 2021) Incidence of PAH: approx. 5 cases per million per year (Maron et al., 2021)

Mean age at diagnosis for PAH: 53 years (Maron et al., 2021)

5-year survival rate for PAH: approx. 58\% (Benza, Miller, et al., 2012; Rådegran et al., 2016)

Prevalence of CTEPH: unclear, because patients at risk are often not assessed appropriately outside the referral hospital setting (Maron et al., 2021)

Cumulative incidence of CTEPH: $0.5 \%$ for general population; 3\% in patients surviving acute pulmonary embolism (Maron et al., 2021)

5-year survival rate for inoperable CTEPH: 53-69\% (Boucly et al., 2017; Quadery et al., 2018; Rådegran et al., 2016) 


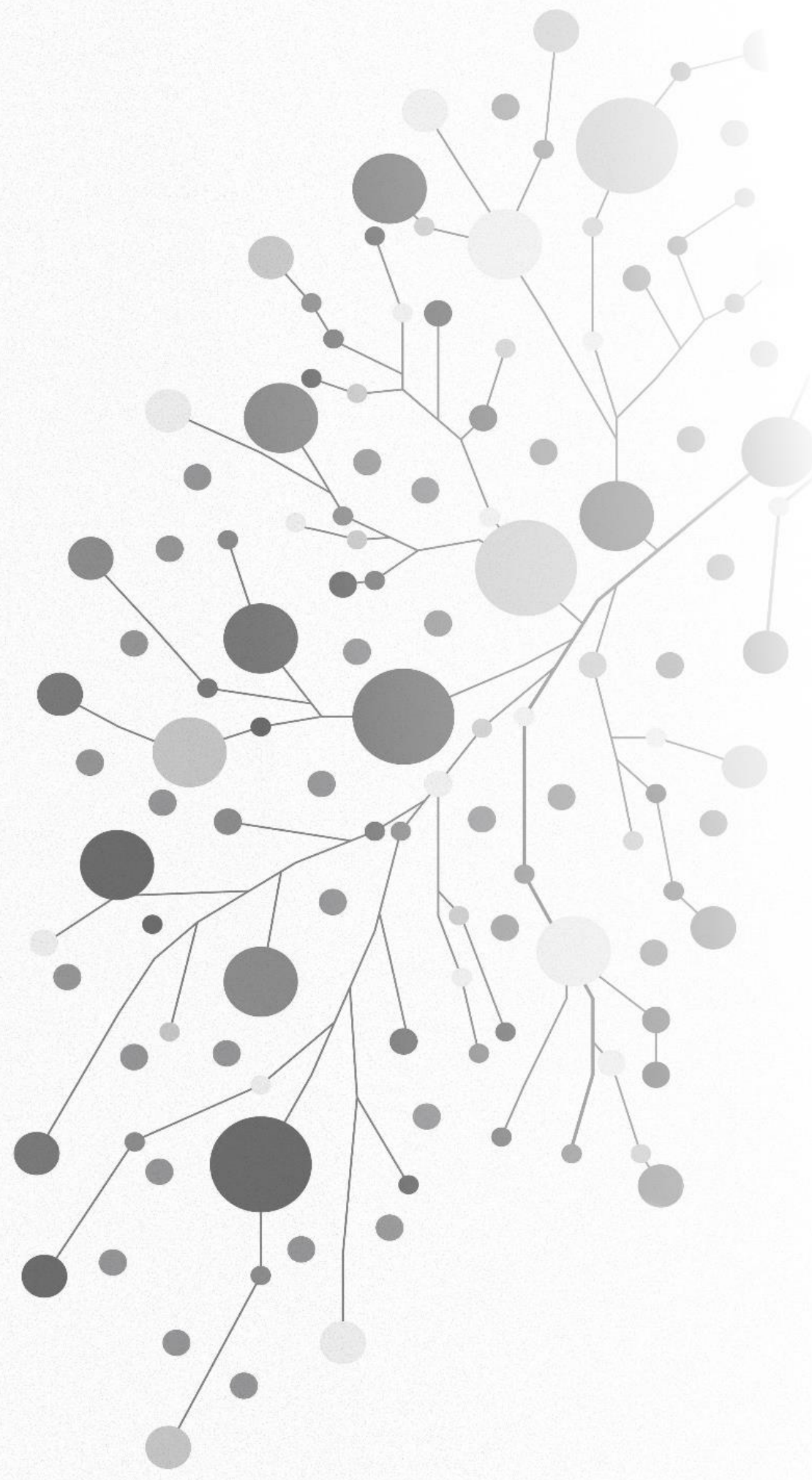


2 Increasing quality of life in pulmonary arterial hypertension: is there a role for nutrition?

Paulien Vinke ${ }^{1}$, Suzanne M. Jansen ${ }^{2}$, Renger F. Witkamp ${ }^{1}$, Klaske van Norren ${ }^{1}$

\section{Affiliations:}

${ }^{1}$ Nutrition \& Pharmacology Group, Division of Human Nutrition and Health, Wageningen University, Wageningen, the Netherlands

${ }^{2}$ Actelion Pharmaceuticals Nederland B.V. Woerden, the Netherlands

Published in: Heart Failure Reviews. 2018; 23(5):711-722.

doi: 10.1007/s10741-018-9717-9 


\begin{abstract}
Pulmonary arterial hypertension (PAH) is a progressive disease primarily affecting the pulmonary vasculature and heart. PAH patients suffer from exercise intolerance and fatigue negatively affecting their quality of life. This chapter summarizes current insights in the pathophysiological mechanisms underlying PAH. It zooms in on the potential involvement of nutritional status and micronutrient deficiencies on PAH exercise intolerance and fatigue, also summarizing the potential benefits of exercise and nutritional interventions.

Pubmed/Medline, Scopus and Web of Science were searched for publications on pathophysiological mechanisms of $\mathrm{PAH}$ negatively affecting physical activity potential and nutritional status, and for potential effects of interventions involving exercise or nutritional measures known to improve exercise intolerance.

It was found that pathophysiological processes that contribute to exercise intolerance and impaired quality of life in $\mathrm{PAH}$ patients include right ventricular dysfunction, inflammation, skeletal muscle alterations and dysfunctional energy metabolism. PAH-related nutritional deficiencies and metabolic alterations have been linked to fatigue, exercise intolerance and endothelial dysfunction. Available evidence suggests that exercise interventions can be effective in PAH patients to improve exercise tolerance and decrease fatigue. By contrast, knowledge on the prevalence of micronutrient deficiencies and the possible effects of nutritional interventions in PAH patients is limited.

So, although data on nutritional status and micronutrient deficiencies in PAH are scarce, the available knowledge, including that from adjacent fields, suggests that nutritional intervention to correct deficiencies and metabolic alterations may contribute to a reduction of disease burden.
\end{abstract}




\subsection{Introduction}

Pulmonary arterial hypertension $(\mathrm{PAH})$ is a progressive disease affecting the arteries of the lungs. It is defined by a mean pulmonary artery pressure $\geq 25 \mathrm{~mm} \mathrm{Hg}$ at rest, an end-expiratory pulmonary artery wedge pressure (PAWP) $\leq 15 \mathrm{~mm} \mathrm{Hg}$ and a pulmonary vascular resistance $>3$ Wood units. There are different etiologies suggested for $\mathrm{PAH}$, as the disease: 1) can develop idiopathically (without a known cause), 2) be heritable (e.g. result from inherited mutations in genes coding for specific proteins like bone morphogenic protein receptor 2), 3) be associated with certain diseases like connective tissue disease, congenital heart disease, portal hypertension or HIV or 4) it can be induced by drugs or toxins (Galiè, Humbert, et al., 2015). The prevalence of all types of pulmonary arterial hypertension (PAH) in Europe is estimated to be in the range of $15-100$ cases per million population. Prognosis of $\mathrm{PAH}$ is poor, with 1- and 3-year survival rates of $87 \%$ and $67 \%$, respectively (Alami et al., 2016; Galiè, Humbert, et al., 2015). The guidelines for the diagnosis and treatment of pulmonary hypertension from the European Society of Cardiology (ESC) and the European Respiratory Society (ERS) describe several risk stratification criteria for determination of prognosis of PAH patients (1-year mortality) (Galiè, Humbert, et al., 2015). Predictors of poor prognosis include advanced functional class, poor exercise capacity and right ventricular dysfunction (Galiè, Humbert, et al., 2015). In heart failure (CHF) patients, right ventricular dysfunction and exercise intolerance are known to contribute to muscle wasting (Kawamoto et al., 2015). Many of the clinical characteristics of PAH such as heart failure, exercise intolerance, functional class, muscle wasting and comorbidities are strongly associated with each other. These characteristics have also been taken along in the REVEAL score, which has been developed to predict disease progression and survival of PAH patients using prognostic criteria (Benza, Gomberg-Maitland, et al., 2012).

Malnutrition and muscle wasting are known clinical manifestations of cachexia, which is often seen in PAH as well (Le Roux et al., 2005). Cachexia as such also contributes to a higher mortality risk and poorer quality of life (Kawamoto et al., 2015; Valentova et al., 2013). At the same time, there appears to be hardly any knowledge on the relevance of nutritional status in PAH. For those readers less familiar with some of the terminology used, box 1 provides some formal definitions 
of cachexia, sarcopenia, nutritional and micronutrient deficiency (Society on Sarcopenia Cachexia and Wasting Disorders, 2018). Interestingly, in case of CHF, which to some degree shows overlapping pathology with $\mathrm{PAH}$, nutritional supplementation with fish oil rich in n-3 fatty acids has shown to improve disease progression. In addition, increased protein intake has proven to be effective in attenuating muscle wasting in CHF patients (von Haehling et al., 2017). Specifically in PAH patients, iron deficiency is known to have a negative impact on exercise tolerance and fatigue (Galiè, Humbert, et al., 2015; Ruiter et al., 2011). With this in mind we hypothesize that nutrition impacts disease progression and quality of life in case of PAH as well. Furthermore, we envisage an opportunity to improve predictive modeling and quality of life for PAH by including nutritional status as a factor. Given the clinical manifestations of $\mathrm{PAH}$, it is expected that effects resulting from optimizing nutritional status of $\mathrm{PAH}$ patients will at least include improved maintenance of muscle mass and function and ultimately quality of life.

The pathophysiology of PAH involves endothelial dysfunction in the pulmonary vasculature, metabolic shifts in vascular cells, hypertrophy and proliferation of smooth muscle cells and unprogrammed growth of neointimal, medial and adventitial layers, leading to thickening and occlusion of the small and mediumsized pulmonary arteries (de Jesus Perez, 2016). Three known pathways involved in PAH are currently used as therapeutic targets. These are the endothelin pathway, the nitric oxide pathway and the prostacyclin pathway (Humbert et al., 2014). Current therapies consist of endothelin receptor antagonists (ERAs), phosphodiesterase 5 (PDE5) inhibitors, a soluble guanylate cyclase (sGC) agonist, prostacyclin, prostacyclin-analogues and a selective IP receptor agonist (Galiè, Humbert, et al., 2015). These compounds aim to reduce pulmonary vascular resistance, provide relief of symptoms, enhance exercise capacity and delay disease progression, but do not cure the disease once it has started. Therefore, additional treatment options for this incurable disease are clearly needed. Among these, treating muscle dysfunction, enhancing physical activity potential and decreasing fatigue could be of great importance. Exercise programs, psycho-social support and dietary consultation are offered to $\mathrm{PAH}$ patients depending on local availability, insurance coverage, laws and regulations. However, there is little information about nutritional status or daily activity of PAH patients, or about possible strategies to 
increase the quality of life of patients by means of nutritional or lifestyle interventions. The ESC/ERS Guidelines for the diagnosis and treatment of pulmonary hypertension (Galiè, Humbert, et al., 2015) contain very limited nutritional and lifestyle recommendations. Therefore, this review aims to gather the information on nutritional status, physical performance and activity levels of PAH patients. In addition, clinical outcomes of previously investigated nutrition and lifestyle interventions are discussed.

Evidence-based nutrition and exercise interventions for PAH are limited, but established lifestyle interventions for CHF might be useful for PAH patients as well based on the similarities in pathophysiology. The 2016 Guidelines of the European Society of Cardiology (ESC) recommend regular aerobic exercise training in patients with heart failure and stable heart failure with reduced ejection fraction to improve functional capacity and reduce the risk of hospitalization (class I, level A recommendation) (Ponikowski et al., 2016). Nutritional advice for heart failure includes avoiding excessive salt intake $(>6 \mathrm{~g} /$ day) and fluid restriction in case of severe heart failure (Ponikowski et al., 2016; von Haehling et al., 2017). Supplementation of omega-3 polyunsaturated fatty acids (N3 PUFA), has shown beneficial effects on mortality and hospitalization in patients with heart failure. Furthermore, high caloric, protein-rich nutritional supplements or branched-chain amino acids can be beneficial in case of muscle wasting or cachexia (von Haehling et al., 2017). Further research is needed to investigate whether these recommendations are also beneficial for $\mathrm{PAH}$ patients. 


\section{Box 2. Formal definitions of used nutritional terminology}

Cachexia: loss of lean tissue mass, involving a weight loss greater than $5 \%$ of body weight in 12 months or less in the presence of chronic illness or as a body mass index (BMI) lower than $20 \mathrm{~kg} / \mathrm{m}^{2}$. According to the definition of Evans et al, three of the following five criteria are also required: decreased muscle strength, fatigue, anorexia, low fat-free mass index, increase of inflammation markers, anemia or low serum albumin

Sarcopenia: loss of muscle mass and function, especially muscle strength and gait speed, commonly associated with aging

Nutritional deficiency: an inadequate supply of essential nutrients from the diet, potentially resulting in malnutrition and disease.

Micronutrient deficiency: an inadequate presence of vitamins or minerals in the body, potentially leading to symptoms or increasing general disease risk. Nutritional status: the condition of the body with respect to factors influenced by the diet: the levels of nutrients in the body and the ability to maintain normal metabolic function.

\subsection{Methods}

We used Pubmed/Medline, Scopus and Web of Science to search for publications connecting the pathophysiological mechanisms of $\mathrm{PAH}$ to impaired physical activity, exercise intolerance, chronic fatigue and nutritional deficiencies often observed in patients with PAH. We also searched for nutritional and exercise interventions to prevent or modulate these symptoms. For the literature search, we used specific combinations of the following search terms: pulmonary arterial hypertension, $\mathrm{PAH}$, pathophysiology, symptoms, mechanism, right ventricular failure, skeletal muscle alterations, inflammation, hypoxia, dysfunctional energy metabolism, lipid accumulation, insulin resistance, satiety hormones, nutritional status, nutrition, deficiency, vitamin, mineral, protein, lipid, fatty acid, carbohydrate, iron, magnesium, calcium, vitamin D, omega 3 fatty acids, omega-3 PUFA, docosahexaenoic acid (DHA), eicasopentaenoic acid (EPA), alpha-linolenic acid (ALA), activity, daily activity, lifestyle, exercise tolerance, exercise capacity, exercise intervention and quality of life. 


\subsection{Activity level and physical performance of PAH patients}

A limited number of studies available underline the prevailing clinical idea that activity levels of patients decline with disease progression (Alami et al., 2016; Pugh et al., 2012; Ulrich et al., 2013). A study using accelerometry to estimate activity levels in $23 \mathrm{PAH}$ and Chronic Thromboembolic Pulmonary Hypertension (CTEPH) patients found a relationship between activity and disease severity. Severely impaired patients (mean pulmonary arterial pressure (mPAP) $50 \pm 7 \mathrm{mmHg}$ ) were inactive for longer periods during the night, and were less active during the day than modestly impaired patients (mPAP $33 \pm 7 \mathrm{mmHg}$ ) (Ulrich et al., 2013). Long nocturnal rest and reduced activity during the day was also reported to correlate with an overall poorer prognosis (Ulrich et al., 2013). Another study showed that PAH patients spent significantly more time in sedentary activities than matched healthy controls and that this impacted all levels of physical activity (Pugh et al., 2012). A more recent paper of Alami et al. supports these findings, reporting that patients with PAH experience such an increase in symptoms like shortness of breath and fatigue, that they have increased problems performing normal daily tasks such as body care, household chores, parenting and leisure activities during progression of the disease (Alami et al., 2016). There are currently more studies underway using activity tracking, such as the wearable wrist device Actigraph, that enable better recording of the activity pattern of PAH patients and the impact of treatment on activity levels (e.g. the TRACE study, clinicaltrials.gov identifier NCT03078907).

\subsection{Nutritional status of PAH patients}

PAH eventually leads to right heart failure, which is found to contribute to loss of skeletal muscle mass (Kawamoto et al., 2015; Valentova et al., 2013). Loss of skeletal muscle is a strong predictor of death in (left) heart failure patients (Anker et al., 1997). Next, loss of skeletal muscle mass is often accompanied with loss of weight and fat mass (Kawamoto et al., 2015; Valentova et al., 2013). Muscle loss in general, diagnosed as sarcopenia or sometimes as cachexia is related to a worsened prognosis and lower quality of life of chronically ill patients (Loncar et al., 2016; von Haehling et al., 2017). The Society on Sarcopenia, Cachexia and Wasting Disorders (SCWD) defines cachexia as "a loss of lean tissue mass, involving a weight loss greater than $5 \%$ of body weight in 12 months or less in the presence of chronic illness or as a body mass index (BMI) lower than $20 \mathrm{~kg} / \mathrm{m} 2$ ". Often three of the following five criteria are 
also required: decreased muscle strength, fatigue, anorexia, low fat-free mass index, increase of inflammation markers, anaemia or low serum albumin (Society on Sarcopenia Cachexia and Wasting Disorders, 2018). Sarcopenia focuses on muscle loss and is defined as "loss of muscle mass and function, especially muscle strength and gait speed, associated with aging" (Society on Sarcopenia Cachexia and Wasting Disorders, 2018), (see also box 1). Improving nutritional status during the earliest stages of chronic diseases such as heart failure is an important factor in reducing the rate at which muscle wasting develops. Despite this, only few studies have investigated nutritional status in PAH. In 2015, Kawamoto showed a close relationship between PAH with Inferior Vena Cava (IVC) dilatation, poor nutritional status and low BMI in a small prospective study with 8 PAH patients (Kawamoto et al., 2015). An older, cross-sectional study with 20 heart failure patients showed that these patients have an $18 \%$ higher resting metabolic rate and risk to develop sarcopenia than healthy age-matched controls (Poehlman, 1994). Based on the similarities in pathophysiology, it could be hypothesized that PAH patients also have an increase in energy expenditure, although future studies will have to confirm this. Furthermore, drugs used to treat $\mathrm{PAH}$, specifically epoprostenol, prostacyclin analogues, and other drugs that target the prostacyclin pathway, are known to induce side-effects that may impact nutritional status and exercise tolerance: nausea, loss of appetite, vomiting, diarrhea, jaw pain, musculoskeletal pain and fatigue (Alami et al., 2016).

\subsection{Disease-related characteristics influencing daily activity or nutritional status}

\subsubsection{Right ventricular failure}

In PAH patients the pulmonary vascular system is obstructed and less elastic, which leads to an increased afterload of the right ventricle with decreased right ventricular cardiac output (Humbert et al., 2004; Provencher et al., 2006; Sutendra \& Michelakis, 2014). This, in turn, decreases the left ventricular preload which leads to a decreased left ventricular output. The decreased left ventricular output causes a reduced oxygen supply to the muscles both at rest and during exercise, contributing to exercise intolerance (Holverda et al., 2006; Laskey et al., 1993; Nootens et al., 1995; Riley et al., 2000; Sun et al., 2001). In addition to a reduced cardiac output, it has been suggested that PAH is associated with impaired chronotropic capacity (Deboeck et al., 2004; Provencher et al., 2006), which is associated with downregulation of $\beta$ - 
adrenoreceptor activity in the right ventricle (Bristow et al., 1992). These effects prevent adequate adaptation of cardiac output and systemic blood pressure during exercise, which can further contribute to exercise intolerance. The pathological changes in the heart and other organs and their contribution to exercise intolerance are well described elsewhere (Tran et al., 2018), and include cardiac factors (right ventricular function), skeletal muscle dysfunction (decreased oxidative enzymes, reduced muscle fiber size), pulmonary factors (endothelial dysfunction, arterial stiffness) and other factors (e.g. inflammation and oxidative stress). Based on this, it is clear that right ventricular failure plays an important role in the exercise intolerance often found in $\mathrm{PAH}$ patients and that the responsible pathophysiological changes are relatively well studied.

\subsubsection{Skeletal muscle alterations}

Multiple causes of reduced daily activity of PAH patients have been proposed. In addition to shortness of breath and fatigue, skeletal and respiratory muscle alterations have also been described. These include a switch from type I fiber to type II fiber in skeletal muscle, increased muscle protein degradation, a reduced muscle capillary density, lower aerobic enzyme activity, mitochondrial abnormalities and impaired calcium homeostasis (Mainguy et al., 2010; Malenfant et al., 2015; Marra et al., 2015; Tonelli et al., 2012). The cross-sectional area and force-generating capacity of muscle cells from the left ventricle have been reported to be reduced by $30 \%$ and $25 \%$, respectively (Manders et al., 2014). Furthermore, forearm muscle weakness was found to correlate with respiratory muscle strength and exercise capacity, independent of hemodynamic severity in a prospective study with 24 idiopathic PAH (IPAH) patients (66\% female) (Bauer et al., 2007). Pathological changes and reduced strength in the diaphragm muscle of $\mathrm{PAH}$ patients or in animal models of PH have been found by several authors (de Man et al., 2011; Manders et al., 2016; Meyer et al., 2005). The changes in the diaphragm muscle might be different from those in skeletal muscle. Instead of a type I to type II fiber switch as seen in skeletal muscle, a switch from type II fiber to type I fiber was observed in the diaphragm of animal models of heart failure (Bowen, Brauer, et al., 2017; Mangner et al., 2015). Alterations in the skeletal and respiratory muscles contribute to exercise intolerance in PAH patients. Whether changes in fiber type in skeletal and respiratory muscles in models of PAH are different (as was found in animal models of heart failure) 
remains to be studied. There is also a lack of studies into the potential effect of lifestyle interventions such as nutrition or exercise on skeletal muscle alterations in PAH.

\subsubsection{Inflammation}

Altered immune function and chronic inflammation are increasingly recognized features of PAH (Cracowski et al., 2014; de Jesus Perez, 2016; Hassoun, 2014; Price et al., 2012). In PAH patients, an accumulation of perivascular inflammatory cells including mast cells, macrophages, dendritic cells and $\mathrm{T}$ and $\mathrm{B}$ lymphocytes has been found in the pulmonary vasculature and lung tissue (Rabinovitch et al., 2014). In addition, chronically elevated serum levels of the pro-inflammatory cytokines IL-1b, IL-6, IL-8, IL-10, MCP-1, fractalkine, CCL5/RANTES and TNF $\alpha$ and chemokines have been reported (Humbert et al., 2004; Price et al., 2012; Rabinovitch et al., 2014). Inflammatory conditions such as connective tissue diseases are associated with increased incidence of $\mathrm{PAH}$, indicating that inflammation might play a significant role in the development and progression of PAH (Humbert et al., 2004; Price et al., 2012). This is supported by findings that in PAH patients, inflammation is associated with worse clinical outcome (Krause et al., 2010; Rabinovitch et al., 2014). Inflammatory cytokines such as IL- 6 and TNF $\alpha$ are known to impact nutritional status and to induce muscle wasting in other chronic diseases (Bowen et al., 2015; von Haehling et al., 2009). Inflammation induces muscle protein breakdown via multiple mechanisms, such as via increased protein degradation through the ubiquitin-proteasome-system (UPS), mitochondrial dysfunction and autophagy (Bowen et al., 2015; von Haehling et al., 2007, 2009). The role of inflammation on mitochondrial dysfunction in chronic heart failure seems two-fold: IL-6 has shown to prevent mitochondrial dysfunction in cardiomyocytes, whereas TNF $\alpha$ induces mitochondrial dysfunction in the same cell-type (Rosca \& Hoppel, 2013). It has been found that TNF $\alpha$ worsens endothelial function in CHF, causing a decreased blood supply to other organs such as the skeletal muscle and the gut. As a consequence, less nutrients are absorbed from the gut and transported to other organs. At the same time, IL-6 induces the acute phase inflammatory response, which requires essential amino acids. Skeletal muscle is an important source of essential amino acids when delivery via the food is limited. Therefore, more skeletal muscle is broken down (von Haehling et al., 2007). TNF $\alpha$, IL-6 and other inflammatory cytokines induce 
transcription of atrogenes, such as MuRF1 and MAFbx/Atrogin-1, via FOXO transcription factors. The E3-ubiquitin ligases MuRF-1 and MAFbx/Atrogin-1 are the main ligases in skeletal muscle that identify proteins for removal via the UPS. The UPS gets activated, which leads to increased protein degradation (Bowen et al., 2015). Proinflammatory cytokines such as TNF $\alpha$ and IL-1 also inhibit food intake via an anorexigenic effect in the brain (Langhans \& Hrupka, 1999).

Based on this information, it can be hypothesized that inflammation decreases nutritional status in $\mathrm{PAH}$ patients and that it can induce muscle wasting or cachexia. This, in turn, supposedly augments the response to inflammation. In this way, a vicious cycle can develop that can only be interrupted when both inflammation and nutritional status are targeted at the same time (Don \& Kaysen, 2004; Laveneziana \& Palange, 2012; McMillan, 2009). Further research is needed to investigate to what extent alterations in immune function and inflammatory processes observed in $\mathrm{PAH}$ could affect nutritional status and whether nutritional supplementation might alter the inflammatory process in PAH.

\subsubsection{Dysfunctional cellular energy metabolism}

Oxygen is important for mitochondrial ATP production via oxidative phosphorylation. In PAH patients, abnormalities in energy production, heme synthesis and mitochondrial function have been hypothesized to result from chronic hypoxia (Archer et al., 2013; Ruiter et al., 2011). A factor important for these impairments is hypoxia-induced factor $1 \alpha$ (HIF-1 $1 \alpha$ ): a hypoxia-sensitive transcription factor. Under normoxic circumstances, HIF-1 $\alpha$ is very unstable and rapidly degraded. Under chronic hypoxic conditions, as occurring in $\mathrm{PAH}$ patients, HIF- $1 \alpha$ stabilizes and moves to the nucleus to induce expression of HIF target genes (Veith et al., 2016). Expression of these genes promotes the delivery of oxygen to tissues and maintenance of ATP levels (Mateo et al., 2003). However, chronic activation of HIF also induces proteins that alter energy metabolism, cell proliferation and cause vascular remodeling, resulting in the development of pulmonary arterial remodeling and pulmonary hypertension (Veith et al., 2016). HIF- $1 \alpha$ activates glycolytic genes increasing the production of lactate from pyruvate and inactivates enzymes necessary for mitochondrial oxidative phosphorylation (Peng et al., 2016). This process is known as aerobic glycolysis and often leads to an energy deficit (Liu et al., 2017; Peng et al., 2016). An increase in lactate levels might 
even suppress food intake, because lactate has an appetite-inhibiting effect (Bray, 2000). The production of high amounts of lactate could also contribute to dysfunction of the right ventricle (Ryan \& Archer, 2014). Due to the metabolic shift from oxidative to glycolytic metabolism, the mitochondrial membrane gets hyperpolarized, leading to proliferation of pulmonary artery smooth muscle cells (PASMC) and reduced apoptosis. This process may contribute to the development of pulmonary hypertension (Peng et al., 2016).

Other factors that could contribute to mitochondrial dysfunction in PAH are deficiencies in caveolin-1 and mutations in BMPRII. Mutated BMPRII shifts glucose metabolism of pulmonary arterial endothelial cells (PAECs) to anaerobic glycolysis, causing decreased ATP production, mitochondrial DNA damage and apoptosis of endothelial cells (Peng et al., 2016). A deficiency in caveolin-1 leads to activation of endothelial nitric oxide synthase (eNOS) and the development of ROS, also contributing to impaired mitochondrial functioning (Peng et al., 2016). Both hypoxia and a decrease in cellular ATP can induce muscle breakdown and dysfunction which further reduces the exercise capacity of the patient (Marra et al., 2015). In summary, due to chronic hypoxia present in $\mathrm{PAH}$, there are changes in mitochondrial energy metabolism that lead to inefficient ATP production in the cells. Lactate, which occurs as a side-product of aerobic glycolysis, might contribute to right ventricular dysfunction and potentially leads to a reduced dietary intake, thereby negatively influencing the clinical status of the patient. However, future studies have to test this hypothesis. A lower cellular ATP production might also induce muscle breakdown and dysfunction itself, although more studies to support this hypothesis are needed.

\subsubsection{Lipid accumulation}

In the healthy human heart, the major source of energy (60-90\%) is provided by fatty acid oxidation (FAO). The other 10-40\% mainly comes from glucose oxidation (Ryan \& Archer, 2014). In PAH patients, due to the Warburg effect, glucose utilization in the heart increases, while FAO is reduced. Despite this, there is an increase in uptake of fatty acids by the fatty acid (FA) transporter CD36 (Talati et al., 2016; Talati \& Hemnes, 2015). Lipid accumulation in cardiomyocytes and skeletal muscle cells is therefore a common feature in PAH patients (Brittain et al., 2016). Cytosolic accumulation of FA leads to a more abundant production of long-chain acyl-CoA in the cytoplasm, which can be converted into intracellular lipid intermediates, such as 
triglycerides, diacylglycerol and ceramides, causing cardiac lipotoxicity. These intermediates induce insulin resistance due to multiple mechanisms, such as impairing pyruvate dehydrogenase (PDH), inhibiting insulin receptor substrate 1 (IRS-1) and glucose transporter type 4 (GLUT4) expression (Talati \& Hemnes, 2015). Next to insulin resistance, the lipid intermediates are also related to the development of cardiac dysfunction and right heart failure (Talati \& Hemnes, 2015). Insulin resistance is related to skeletal muscle weakness in CHF patients, although it is unclear whether this relationship is causal (Doehner et al., 2015). Cardiac dysfunction and right heart failure lead to fatigue and hypoxia, which both contribute to exercise intolerance. This altogether leads to muscle protein breakdown. The combination of immobility and the inability to use fat can lead to a sarcopenic obesity phenotype (high BMI, low muscle mass), a malnourished phenotype that is also more and more encountered in cancer (Carneiro et al., 2016) and is associated with increased morbidity and mortality in this disease. Based on the above described mechanisms and cause-effect relationships between lipid intermediates, insulin resistance and skeletal muscle weakness, the prevalence of sarcopenic obesity in PAH patients needs further investigation.

\subsubsection{Insulin resistance}

A higher prevalence of insulin resistance has been reported in PAH patients than in the general population, and this may be a disease modifier or risk factor for PAH (Hansmann et al., 2007; Naderi et al., 2014; Pugh et al., 2011; Zamanian et al., 2010). Insulin resistance is known to be a risk factor for cardiovascular disease (CVD) in general. It has been linked to diseases that share pathophysiological characteristics with PAH such as idiopathic cardiomyopathy or congestive heart failure (Zamanian et al., 2010). Inflammatory cytokines, seen in the pathogenesis of PAH, are likely to contribute to insulin resistance (Paulin \& Michelakis, 2014). Obesity in PAH patients may contribute to the development of insulin resistance if coupled with low daily activity levels (Zamanian et al., 2010), but insulin resistance has been found independently of obesity in PAH patients as well (Pugh et al., 2011). In general, insulin resistance induces energy deficit in the muscle, which triggers muscle protein breakdown. From this, it becomes clear that there are many observed associations between insulin resistance and $\mathrm{PAH}$, but little is known about whether it is a symptom of, or a contributing factor to, the disease. More research is needed 
to show whether insulin or glucose levels play a role in the pathophysiology of $\mathrm{PAH}$ and whether it contributes to a loss of muscle mass in PAH patients.

\subsubsection{Gut-derived satiety hormones}

Deregulations in the expression of satiety hormones, such as increased levels of the anorexigenic hormone PYY after a meal, have been found in a small study with 9 cardiac cachectic patients with primary PAH (Le Roux et al., 2005). In addition to its systemic effects, PYY reduces digestion rate by decreasing GI motility, gall-bladder emptying and gastric emptying, which further contributes to a reduction in food intake (Luttikhold et al., 2013). Larger trials should show whether an increase in the anorexigenic hormone PYY or changes in other gut-derived satiety hormones after a meal contribute to reduced food intake in PAH patients.

\subsection{Disease-related nutritional deficiencies and intervention trials investigating their possible connection to quality of life}

\subsubsection{Iron deficiency}

Iron deficiency is common in patients with idiopathic and heritable forms of $\mathrm{PAH}$. The prevalence has been estimated to be between 30 and $65 \%$, dependent on the type of PAH and status of the patient (Galiè, Humbert, et al., 2015; Rhodes, Wharton, et al., 2011; Ruiter et al., 2011; Soon et al., 2011). Iron deficiency is defined by reduced serum iron (normal= 9.0-30.0 $\mu \mathrm{mol} / \mathrm{L}$ ), serum ferritin (normal= 3-400 $\mu \mathrm{g} / \mathrm{L}$ (males), $30-150 \mu \mathrm{g} / \mathrm{L}$ (females)) and transferrin saturation (normal= 16-45\%) (Viethen et al., 2014). In patients with IPAH, a low iron status has been related to a reduced exercise capacity, measured as the 6 minute walk test, and to poorer survival (Ruiter et al., 2011, 2015). Iron deficiency was reported to be independent of the presence of anemia (Robinson et al., 2014; Ruiter et al., 2011; Soon et al., 2011) and not associated with a worsened right ventricle function (Van Empel et al., 2014). Availability of iron influences the pulmonary vasoconstrictor response to hypoxia and the basal pulmonary artery pressure (Balanos et al., 2002; Smith et al., 2008). Iron status influences modulation of the pulmonary circulation, but also myocardial and skeletal muscle function. Iron is essential for oxygen transport and functions as a cofactor in several mitochondrial oxidative enzymes and the respiratory chain. Inhibition of dietary iron uptake by the negative regulator of plasma iron levels, hepcidin, might form a mechanism behind the iron deficiency. The main iron 
transporter of the intestine (ferroportin) is inhibited by hepcidin, resulting in a decreased intestinal absorption of iron. Hepcidin levels are higher in patients with PAH. Moreover, a low intestinal absorption of iron is supported by reports that the response to oral iron supplementation is low in PAH patients (Rhodes, Wharton, et al., 2011; Ruiter et al., 2015). High hepcidin levels might be due to dysfunctional BMPRII signaling and/or the presence of chronic inflammation. Normally, high hepatic iron levels induce bone morphogenic protein 6 (BMP6) expression, which stimulates hepcidin transcription via BMP response elements I and 2 (BMPRI and BMPRII) on the hepcidin gene. Inflammatory cytokines such as IL-1beta and IL-6 stimulate hepcidin transcription via different mechanisms, leading to excessive hepcidin production. IL-1beta might even provide the onset signal, as it induces the transcription of IL-6. This excessive hepcidin production also happens when there are no high hepatic iron levels present and thereby leads to inflammation-induced anemia (Kanamori et al., 2017).

In summary, iron deficiency is common in PAH patients and contributes to the disease itself and to exercise intolerance by influencing the pulmonary circulation, myocardial and skeletal muscle function and oxidative energy metabolism. High levels of hepcidin, induced by inflammatory cytokines and/or dysfunctional BMPRII signaling, lead to inhibition of dietary iron uptake.

\subsubsection{Iron supplementation}

The response to oral iron supplementation in PAH patients is low, which is likely due to inhibition of iron uptake by hepcidin in the digestive tract (Rhodes, Wharton, et al., 2011; Ruiter et al., 2015). Therefore, more recent studies investigated the effect of intravenous infusion of iron in the form of ferric carboxymaltose. For example, Viethen et al. and Ruiter et al. studied the effect of a single dose of $1000 \mathrm{mg}$ ferric carboxymaltose. They used observation periods of 8 and 12 weeks in 20 and $15 \mathrm{PAH}$ patients respectively (Ruiter et al., 2015; Viethen et al., 2014). Both studies found improvement of serum iron status and quality of life, as measured by the patientreported Short Form 36 (SF-36) questionnaire, and showed minimal side effects. The study of Viethen et al. also found an improvement in 6-minute walk distance (6MWD), whereas Ruiter and colleagues did not, although they did find improved endurance exercise capacity and mitochondrial oxidative capacity (Ruiter et al., 2015; Viethen et al., 2014). A larger study into the effects of parenteral iron 
replacement in at least 60 patients with IPAH is underway (The 'Supplementation of Iron in Pulmonary Hypertension' (SIPHON) Phase II clinical trial) (Howard et al., 2013). More randomized controlled trials are needed to show whether parenteral iron replacement is more successful in treating iron deficiency than oral iron supplementation and if this leads to a better quality of life in PAH patients.

\subsubsection{Vitamin D}

Vitamin D deficiency is linked to musculoskeletal, metabolic and cardiopulmonary diseases and to disorders of the immune system (Mirdamadi \& Moshkdar, 2016; Zittermann, 2003). It has been suggested that vitamin D influences smooth muscle cell proliferation and endothelial function, which are both affected in PAH (Atteritano et al., 2016; Demir et al., 2013; Mirdamadi \& Moshkdar, 2016). Pulmonary hypertension was associated with vitamin D3 deficiency in a group of 40 systemic sclerosis patients (Atteritano et al., 2016). In a small prospective uncontrolled longitudinal study, weekly supplementation of 50.000 IU cholecalciferol (vitamin D3) plus a daily dose of $200 \mathrm{mg}$ magnesium, $8 \mathrm{mg}$ zinc and $400 \mathrm{IU}$ vitamin D for a three month period has been found to significantly increase serum vitamin $\mathrm{D}$ level and 6MWD in 22 patients with pulmonary hypertension and vitamin D deficiency. It also led to improvements in right ventricle size and function (Mirdamadi \& Moshkdar, 2016). While the evidence from this single study is limited, it does show an interesting potential of correcting vitamin D deficiencies to improve quality of life in PAH patients. Further rigorously conducted randomized trials into the effect of vitamin D supplementation should confirm potential effects on the right ventricle, exercise tolerance and quality of life.

\subsection{Exercise interventions}

In the past, exercise training was not recommended in patients with PAH. It was thought that exercise could be a major risk due to increased blood flow, a drop in cardiac output that may worsen right ventricular function or risk of arrhythmia and hypoxia (Arena et al., 2015; Madonna et al., 2016; Zafrir, 2013). However, more and more studies now show that exercise training can actually be safe and beneficial for exercise capacity, peak oxygen capacity, systemic pulmonary artery pressure, heart rate and quality of life in patients with various forms of pulmonary hypertension 
(Becker-Grünig et al., 2013; Chia et al., 2017; Grünig et al., 2011; Grünig, Lichtblau, et al., 2012; Grünig, Maier, et al., 2012).

There are different kinds of exercise training that are used in cardiac and pulmonary diseases: 1 ) aerobic exercise training, like cycling or walking; 2 ) strength training and 3) respiratory exercise training (Chia et al., 2017). Respiratory training often consists of body perception, yoga and respiratory muscle strengthening exercises (Madonna et al., 2016). Although yoga and meditative breathing have been mentioned as possible interventions, studies exclusively examining these interventions were so small that no conclusions can be drawn from them (Awdish et al., 2015). Studies on exercise training in $\mathrm{PAH}$ patients were almost exclusively done in medically stable patients. González-Saiz and colleagues found that aerobic, inspiratory and muscle resistance training during a short intervention period of 8 weeks can improve muscle power and strength in $\mathrm{PAH}$ patients. Also peak $\mathrm{VO} 2$ was improved by this exercise intervention (González-Saiz et al., 2017). Others found that the combination of aerobic exercise and education can improve fatigue and daily activity, while this effect was not found when giving education only (Weinstein et al., 2013). Different reviews have been published that summarize the effect of exercise interventions combining aerobic exercise, resistance exercise and/or respiratory training during periods of 3-15 weeks. Sessions were often performed between 2-5 times per week. Amongst other, improvements have been found in $6 \mathrm{MWD}$, fatigue, peak VO2, QoL, increased amount of capillaries per muscle fiber, mean pulmonary blood volume, lower resting heart rate and increased strength of different muscles (including the respiratory muscles) (Arena et al., 2015; Babu et al., 2016; Buys et al., 2015; Chia et al., 2017; Pandey et al., 2015; Zafrir, 2013). Researchers also reported mild exerciserelated adverse events, like dizziness, desaturation, progressive fatigue towards the end of the training period, (pre-)syncope and supraventricular tachycardia in about $5 \%$ of the participants (Babu et al., 2016; Pandey et al., 2015). It has been found that fainting during exercise could be triggered in $\mathrm{PAH}$ patients when doing isometric exercise training. Therefore these authors recommended not to imply isometric exercise in training schedules (Zafrir, 2013).

According to the Pulmonary Hypertension Association (PHA) PAH patients should seek advice regarding safe exercise training from their doctor before starting to exercise. The PHA recommendations for exercise in patients with PAH state that patients should not over-exercise to the point of dizziness, chest pain or severe 
shortness of breath. Recommended forms of exercise are light resistance training of small muscle groups (without heavy lifting) and light to moderate aerobic activity, such as walking or swimming. For symptomatic PAH patients it is not advised to exercise the upper and lower body at the same time. Patients with severe exercise intolerance or those with a history of fainting or dizziness are not advised to continue or start a regular exercise program. Lastly, exercise is better avoided during extreme weather circumstances such as very hot or cold temperatures (Pulmonary Hypertension Association, 2008).

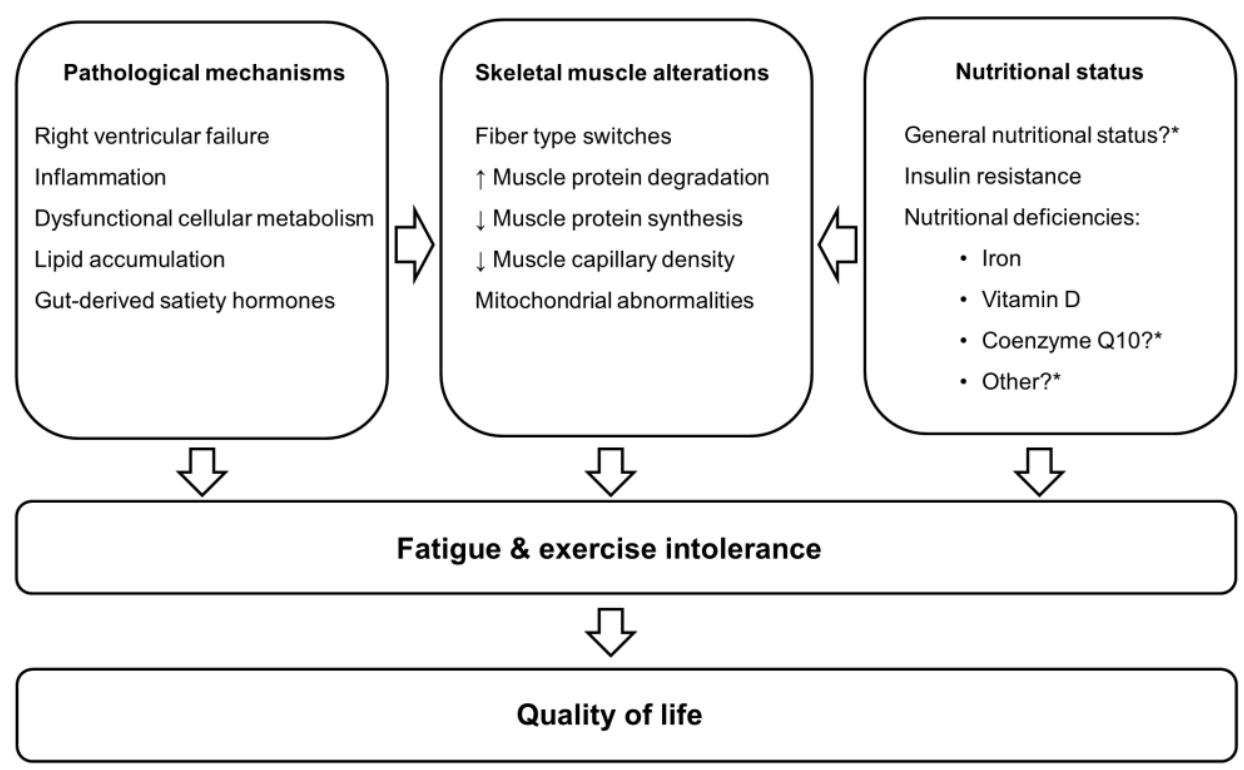

Figure 2.1: Factors influencing skeletal muscle alterations, fatigue, exercise intolerance and quality of life in pulmonary arterial hypertension $\left(^{*}\right.$ current knowledge gaps)

\subsection{Discussion and conclusion}

Nutritional status is likely to be impaired in patients with PAH due to an increased energetic demand, increased protein catabolism and congestion of the splanchnic organs (Alami et al., 2016; Kawamoto et al., 2015; Poehlman, 1994). The effect and potential side effects of exercise interventions in PAH patients are relatively well studied. Although the pathophysiology of PAH involves many mechanisms that 
may influence nutritional status and may induce muscle wasting (see figure 2.1), potentially limiting the effect of exercise interventions, there is only little scientific knowledge about the nutritional status of PAH patients and the way this may impact physical activity. We found no data on the general nutritional status and only limited data on the daily activity of PAH patients. Although deficiencies in micronutrients such as vitamin D, vitamin B12, iron or magnesium might influence symptoms of fatigue and quality of life, there are only few studies into the prevalence of such nutritional deficiencies in PAH patients. Next to micronutrient deficiencies, the presence of insulin resistance is a sign of metabolic change and can also contribute to feelings of fatigue. All of these nutritional deficiencies and metabolic changes can be monitored and treated. Although it is being recognized that oral iron supplementation in iron deficient $\mathrm{PAH}$ patients is ineffective, there is limited insight in alternative treatments. There are only few studies on the effect of intravenous iron substitution in PAH patients to reduce iron deficiency. Current studies are relatively small with a sample size of 15-20 subjects. Data of a larger study that is currently being performed have not been published yet. Studies on the effect of micronutrients such as vitamin D supplementation in $\mathrm{PAH}$ patients are rare, small in size and study designs can be improved. Chronic inflammation is an important feature in the pathophysiology of $\mathrm{PAH}$, but the effect of nutritional intervention on inflammation status of these patients is unknown. High quality papers on the effect of treatments to reduce adverse effects of medication that impact nutritional status are lacking. It should also not be forgotten that physical inactivity itself induces skeletal muscle atrophy (D'Antona et al., 2003), so prevention of becoming inactive in the first place should receive extra clinical attention.

In conclusion, larger and well-designed studies into the nutritional status of PAH patients, the prevalence of micronutrient deficiencies and the effect of supplementation strategies to reduce these deficiencies and improve quality of life in PAH patients are needed.

\section{Acknowledgements}

We thank Shani Sela and Roos Verstegen for their assistance in the literature search for this review and Marianne Geleijnse and T. Scott Bowen for their critical comments on the manuscript. 


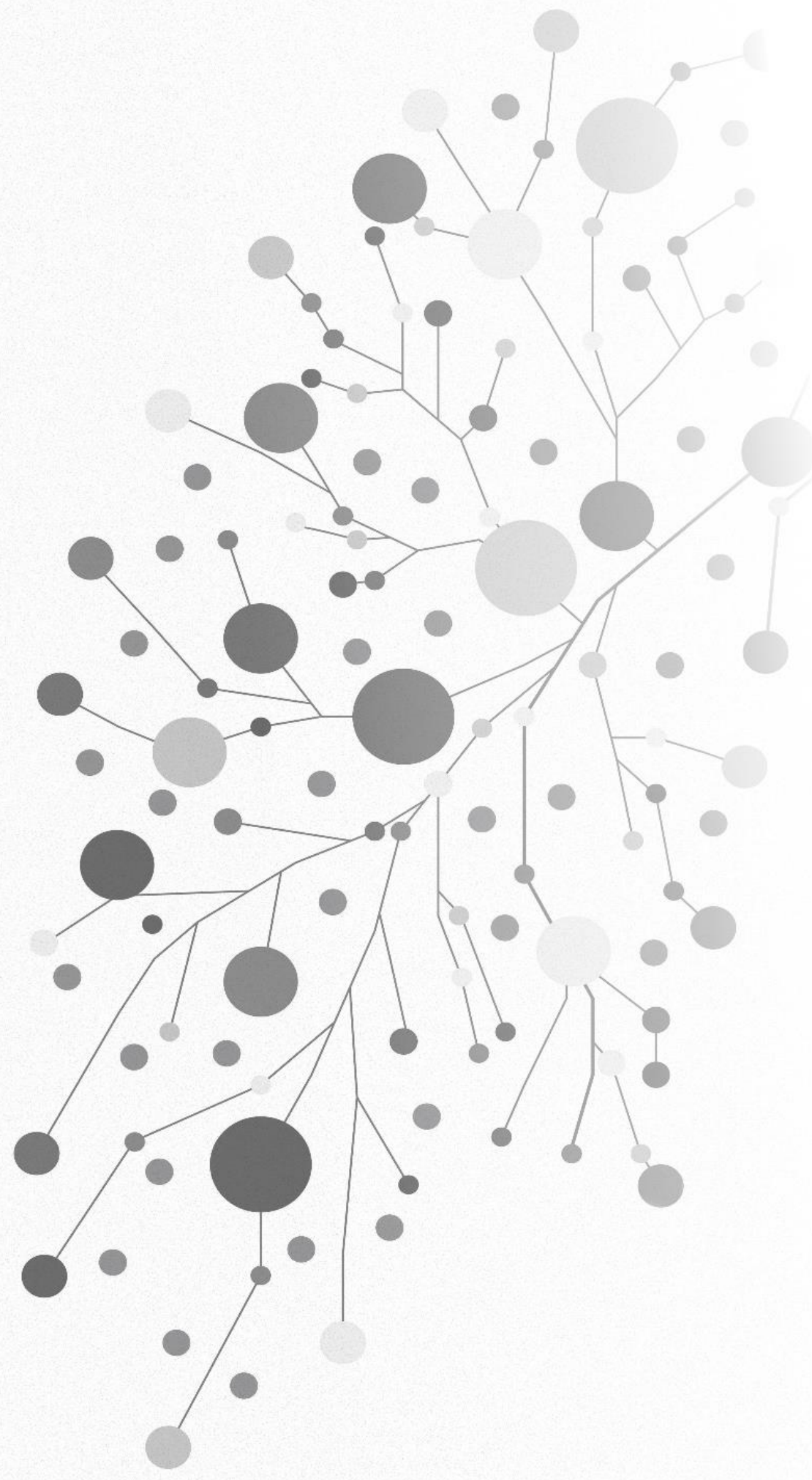


3 Prevalence of micronutrient deficiencies and relation with clinical and patient-related outcomes in pulmonary hypertension type I and IV

Paulien Vinke 1, Thomas Koudstaal 2, Femke Muskens 2, Annemien van den Bosch 2, Michiel

Balvers ${ }^{1}$, Mieke Poland ${ }^{1}$, Renger F Witkamp ${ }^{1}$, Klaske van Norren ${ }^{1}$, Karin A Boomars ${ }^{2}$

\section{Affiliations:}

${ }^{1}$ Nutritional Biology, Division of Human Nutrition and Health, Wageningen University, Stippeneng 4, 6708 WE Wageningen, the Netherlands

2 Department of Pulmonary Medicine, Erasmus MC, University Medical Center Rotterdam, Dr. Molewaterplein 40, 3015 GD Rotterdam, The Netherlands

\# contributed equally

Published in: Nutrients. 2021; 13(11):3923.

doi: 10.3390/nu13113923. 


\begin{abstract}
Pulmonary Hypertension (PH) is a rare progressive and lethal disease affecting pulmonary arteries and heart function. The disease may compromise nutritional status, which impairs physical performance of the patient. This study aimed to determine the prevalence of micronutrient deficiencies in pulmonary arterial hypertension (PAH) and chronic thrombo-embolic pulmonary hypertension (CTEPH) patients, both at diagnosis and after treatment. Moreover, correlations between micronutrients and clinical outcomes were assessed.

Eighty-one blood samples from a prospective observational cohort study were analyzed for concentrations of micronutrients and inflammation-related factors. Samples consisted of newly diagnosed (treatment naive) PAH and CTEPH patients and patients treated for 1.5 years according to the ERS/ESC guidelines.

In the newly diagnosed group, $42 \%$ of $\mathrm{PAH}$ patients and $21 \%$ of CTEPH patients were iron deficient, compared to $29 \%$ of PAH patients and $20 \%$ of CTEPH patients in the treatment group. Vitamin D deficiency occurred in $42 \%$ of the newly diagnosed PAH patients, in $71 \%$ of the newly diagnosed CTEPH patients, in $68 \%$ of the treated PAH patients and in $70 \%$ of the treated CTEPH patients. Iron levels correlated with 6-minute walking distance (6MWD). Vitamin D deficiency was not associated with outcome measurements.

Iron and vitamin D deficiencies are highly prevalent in PAH and CTEPH patients, underlining the need for monitoring their status. Studies evaluating the possible effects of different supplementation strategies for iron as well as vitamin D in PAH and CTEPH patients are necessary.
\end{abstract}




\subsection{Introduction}

Pulmonary hypertension (PH) refers to a heterogeneous group of diseases characterized by vasoconstriction and structural remodeling of the pulmonary arteries. Increased vascular resistance and elevated pulmonary arterial pressures lead to right ventricular hypertrophy and eventually to heart failure and premature death (Hoeper et al., 2013; Simonneau et al., 2019). Symptoms are progressive and include inflammation, right ventricular dysfunction, serious exercise intolerance and fatigue, reducing the quality of life of the patients (Tran et al., 2018; Vinke et al., 2018). Currently, PH is divided into 5 subgroups according to the classification of the World Health Organisation (WHO), European Respiratory Society (ERS) and European Society of Cardiology (ESC) (Galiè, Humbert, et al., 2015). In this study we focused on patients with WHO type 1: pulmonary arterial hypertension (PAH) and type 4: chronic thrombo-embolic pulmonary hypertension (CTEPH), because these patients are typically treated with $\mathrm{PH}$-specific therapies in expert centers. In both categories, the pathophysiology originates in the pulmonary arteries. Prognosis of both PAH and CTEPH is poor, with 3- and 5-year survival rates for PAH of $67 \%$ and 57-59\%, respectively (Benza, Miller, et al., 2012; Galiè, Humbert, et al., 2015; Rådegran et al., 2016), and 5-year survival rates of 53-59\% for inoperable CTEPH (Boucly et al., 2017; Quadery et al., 2018; Rådegran et al., 2016). The prognosis of CTEPH patients with central laesions, who are eligable for and who underwent a pulmonary endarterectomy is improved with 5-years survival rates around $85 \%$ (Sandqvist et al., 2021).

The ESC/ERS guidelines for pulmonary hypertension contain various recommendations for $\mathrm{PH}$ treatment, although very few on nutrition. Moreover, there are no data on the influence of nutritional status on prognostic indicators as defined by the ESC/ERS (Galiè, Humbert, et al., 2015). However, nutritional status, including the occurence of micronutrient deficiencies is likely to be relevant to both the overall physiological status and the exercise capacity of the patient. It is known that micronutrient deficiencies, such as those of magnesium, vitamin D and iron are linked to inflammatory processes, which may contribute to exercise intolerance (van Dronkelaar et al., 2018; Zittermann, 2003). Vice versa, a reduction in physical activity can have consequences for vitamin $\mathrm{D}$ synthesis and might also affect dietary habits. Hence, monitoring and optimization of nutritional status in patients with PH holds 
promise to improve overall wellbeing of $\mathrm{PH}$ patients. Literature research reveals a lack of studies on nutritional deficiencies in PAH and CTEPH patients and identifies important knowledge gaps regarding the potential benefits of nutritional intervention on the quality of life of these patients (Vinke et al., 2018). It has been suggested that anaemia and iron-deficiency are prevalent in CTEPH and in PAH patients in particular (Soon et al., 2011). As with other individuals, these factors are likely to have a negative impact on exercise tolerance and fatigue in PH (Galiè, Humbert, et al., 2015; Ruiter et al., 2011). Compared to iron, even less is known on the status of other relevant micronutrients, including vitamin $\mathrm{D}$ and magnesium in $\mathrm{PH}$ patients. Although magnesium status is relevant for cardiac and skeletal muscle function, glucose homeostasis (de Baaij et al., 2015; Dominguez et al., 2006) and chronic inflammation (Shahi et al., 2019), it is not routinely monitored. Vitamin D deficiency is highly prevalent in Europe during the winter season and in chronically ill patients or elderly also throughout the whole year (Zittermann, 2003). Deficiencies of vitamin D are linked to bone health, muscle dysfunction and attenuated immune function (Bikle, 2014; Zittermann, 2003). On the other hand, inflammation itself can impact vitamin D status, for example via decreasing vitamin D binding protein (DBP), leading to lower 25-hydroxy-vitamin D (25(OH)D) levels in the blood (Waldron et al., 2013). So far, there is limited evidence of potential benefit of correcting vitamin D deficiencies in patients with PAH. In summary, studies into the prevalence of micronutrient deficiencies and their effects on clinical outcomes in PAH and CTEPH patients are currently lacking, although these deficiencies can negatively influence the patient's health status. This would ultimately merit suppletion strategies, although more research would be needed on this.

This study aimed to determine the actual status of micronutrients that may play a role in inflammation, fatigue and exercise intolerance in PAH and CTEPH patients at time of diagnosis and after 1.5 years of conventional treatment. A secondary aim was to explore whether there is a correlation between micronutrient deficiencies and clinical outcome parameters. Although pulmonary arterial hypertension is relatively rare, this study collected data on micronutrient status and many clinical and patientrelated outcome variables of a relatively large group of patients from the Biopulse prospective observational cohort study. 


\subsection{Materials and Methods}

\subsubsection{Patients and study design}

A prospective observational cohort study is conducted in the Erasmus University Medical Centre for specialised PH care since May 2012. Pulmonary hypertension patients $>18$ years old with a mean pulmonary arterial pressure $(\mathrm{mPAP}) \geq 25 \mathrm{mmHg}$, a wedge pressure $\leq 15 \mathrm{mmHg}$ and a pulmonary vascular resistance (PVR) $\geq 3 \mathrm{WU}$ measured by right heart catheterization (RHC) at diagnosis are invited to take part in the study. Diagnosis of PAH and CTEPH patients is made according to the ERS/ECSC guidelines (Galiè, Humbert, et al., 2015; Simonneau et al., 2019) and subclassification according to the WHO classification system. Patient characteristics and $\mathrm{PH}$ subgroups are shown in tables 1 and 2 in the results section. Exclusion criteria were incomplete diagnostic work-up and therefore no confirmed $\mathrm{PH}$ diagnosis, age $<18$ years, or not being capable of understanding or signing informed consent. The study protocol was approved by the medical ethical committee (MEC2011-392). A written informed consent was provided by all patients. The study is performed in accordance with the principles of the Declaration of Helsinki.

\subsubsection{Clinical data collection}

Baseline data were collected during the inpatient screening visit. At baseline, all patients underwent physical examination by a pulmonary physician and a cardiologist, 6-minute walking test (6MWT), pulmonary function tests, VQ scan, chest computed tomography scan, ultrasound of the liver, 12-lead electrocardiography (ECG), echocardiography, venous blood sampling and a RHC. Patient characteristics and vital signs collected at baseline include age, sex, height, weight, systemic blood pressure, heart rate and peripheral oxygen saturation. The NYHA functional class was used to grade the severity of functional limitations. During RHC, a Swan-Ganz catheter was inserted in the internal jugular vein. A standardized protocol for the work-up of $\mathrm{PH}$ was used to obtain hemodynamic measurements. Fick's principle was used to measure cardiac output. If the obtained capillary wedge pressure was ambiguous, a fluid challenge was performed to distinguish pre-capillary PH from $\mathrm{PH}$ due to left heart disease. Data were collected and stored in PAHTool (version 4.3.5947.29411, Inovoltus, Santa Maria da Feira, Portugal), an online electronic case report form. 


\subsubsection{Clinical follow-up}

Patients were treated according to the ERS/ESC guidelines (Galiè, Humbert, et al., 2015). CTEPH patients were assessed for eligibility for either a pulmonary endarterectomy or a balloon pulmonary angioplasty. Patients who underwent one of the above procedures, were not censored afterwards.

All patient were prospectively followed-up by half-yearly scheduled visits to the outpatient clinic. During this visit vital signs were collected, NYHA functional class was graded, venous blood samples were taken (e.g. for measurement of $\mathrm{N}$-terminal prohormone brain natriuretic peptide (NT-pro BNP)), and a 6MWT as well as a echocardiography was performed.

\subsubsection{Venous blood sampling}

Venous blood samples were obtained at baseline and every six months during follow-up. Transfer of the blood samples to the clinical chemistry laboratory occurred within 2 hours from withdrawal. Hemoglobin $(\mathrm{Hb})$ and NT-pro-BNP were directly determined in the fresh blood samples. Serum samples and plasma samples were processed and aliquoted and stored at -80 degrees Celsius until further analysis.

\subsubsection{Laboratory analyses}

Measurement of plasma iron (ferrum), ferritin, transferrin, magnesium, calcium, phosphate, vitamin B12 (cobalamin) and folic acid were performed in serum samples according to standard operating procedures of the clinical chemistry lab of the Erasmus University Medical Center Rotterdam. Vitamin D was analysed using the Fujirebio Lumipulse G1200 system with a Lumipulse 25-OH Vitamin D assay (Fujirebio, catalog number 234020 calibrators and 234013 reagent) according to the manufacturer's instructions. All other analytes were determined using a Cobas 8000 system (Roche). Concentrations of each micronutrient for individual patients were compared with specific reference values as validated by the Erasmus University Medical Center Rotterdam and as specified in tables 5.3-5.5. The reference value for vitamin D was specified as $>50 \mathrm{nmol} / \mathrm{l}$ (Balvers et al. 2015). Patients with concentrations below the reference value were scored as having a too low circulating concentration of that micronutrient. 
Hepcidin values were analysed using a human hepcidin (Hepc) ELISA kit (Cusabio, catalog number CSB-E16062h) in undiluted EDTA plasma samples according to the manufacturers protocol. Vitamin D binding protein was analysed using a GCGlobulin (Vitamin D binding protein) Human ELISA kit (Abcam, catalog number Ab108853) in undiluted EDTA plasma samples according to the manufacturers protocol. Reference values for vitamin D binding protein and hepcidin were specified according to the ELISA kits manufacturers manual.

\subsubsection{Statistics}

Unpaired, two-tailed t-test in Graphpad Prism (version 5) was used to compare groups. Data were presented as mean+SD. Missing data were not replaced in the analysis. Spearman's correlation was performed in Rstudio using the ggscatter function. Significance was accepted as $\mathrm{p}<0.05$.

\subsection{Results}

\subsubsection{Characteristics of participants}

In this study, 33 newly diagnosed patients with PAH and CTEPH are included and 48 patients who were under conventional $\mathrm{PH}$ specific treatment for 1.5 years (treated group). In the newly diagnosed group, 19 patients were diagnosed with PAH and 14 patients with CTEPH. Baseline characteristics do not differ between persons diagnosed with $\mathrm{PAH}$ or CTEPH, except for height (table 3.1). There are no differences in age, weight, body mass index (BMI), 6-minute walking distance (6MWD), right ventricular systolic pressure (RVSP) measured by echocardiography, and mean PAP and cardiac index (CI) at RHC.

As is shown in table 3.2, in the treated group 38 patients with $\mathrm{PAH}$ and 10 patients with CTEPH are included. The mean age of the participants in the CTEPH group is higher than the age of the PAH group (64.5 versus 48.8 years, respectively). This is not surprising since in general PAH patients tend to be younger than CTEPH patients. No differences are observed in height, weight and BMI between patients with PAH and CTEPH in this group. The percentage of females in each group is relatively similar (63\% and $70 \%$, respectively). The $6 \mathrm{MWD}$ is not different between patients with $\mathrm{PAH}$ or $\mathrm{CTEPH}$, both at baseline and after 1.5 years of treatment. Baseline characteristics for RVSP, mean PAP, PVR and CI are shown in table 3.2 for each patient group, as well as RVSP after 1.5 years of treatment. 
Table 3.1: Characteristics of patients in the newly diagnosed group

\begin{tabular}{|c|c|c|}
\hline Characteristic & PAH & СТЕРН \\
\hline n (\%) & $19(58)$ & $14(42)$ \\
\hline Gender, female (\%) & $14(74)$ & $6(43)$ \\
\hline Age, y & $62.5 \pm 14.7$ & $63.9 \pm 12.9$ \\
\hline Height, cm & $164.6 \pm 9.8$ & $174.5 \pm 10.0$ * \\
\hline Weight, kg & $72.9 \pm 18.7$ & $80.1 \pm 17.6$ \\
\hline BMI, kg/m² & $26.7 \pm 5.6$ & $26.4 \pm 5.9$ \\
\hline NYHA class (1:2:3:4) & $0: 5: 11: 1$ & $2: 4: 6: 1$ \\
\hline \multicolumn{3}{|l|}{ Cause of Pulmonary Hypertension } \\
\hline НРАН (\%) & $2(11)$ & \\
\hline IPAH (\%) & $4(21)$ & \\
\hline CTD (\%) & $9(47)$ & \\
\hline $\mathrm{CHD}(\%)$ & $0(0)$ & \\
\hline HIV (\%) & $0(0)$ & \\
\hline Portopulmonary (\%) & $3(16)$ & \\
\hline Drugs/toxins/medication (\%) & $1(5)$ & \\
\hline Other $(\%)$ & $0(0)$ & \\
\hline \multicolumn{3}{|l|}{ Type of CTD related PAH } \\
\hline SSc (\%) & $7(78)$ & \\
\hline SLE (\%) & $1(11)$ & \\
\hline Sjogren (\%) & $1(11)$ & \\
\hline 6MWD, m & $352 \pm 121$ & $447 \pm 240$ \\
\hline Echocardiography, RVSP in mm/Hg & $76.5 \pm 19.9$ & $72.1 \pm 21.0$ \\
\hline \multicolumn{3}{|l|}{ R-catheterisation } \\
\hline Mean PAH, mmHg & $46.5 \pm 14.0$ & $42.0 \pm 11.9$ \\
\hline PVR, WU & $7.3 \pm 3.3$ & $6.2 \pm 3.2$ \\
\hline $\mathrm{CI}$ & $2.7 \pm 0.7$ & $2.8 \pm 0.8$ \\
\hline
\end{tabular}

$\left.{ }^{*}: \mathrm{p}<0.05,{ }^{* *}: \mathrm{p}<0.01\right)$ compared to the PAH group 
Table 3.2: Characteristics of patients in the treated group

\begin{tabular}{|c|c|c|}
\hline Characteristic & PAH & СТЕРН \\
\hline n (\%) & $38(79)$ & $10(21)$ \\
\hline Gender, female (\%) & $24(63)$ & $7(70)$ \\
\hline Age, y & $48.8 \pm 15.1$ & $64.5 \pm 12.0$ ** \\
\hline Height, cm & $168.7 \pm 10.8$ & $169.8 \pm 6.3$ \\
\hline Weight, kg & $75.6 \pm 18.4$ & $83.3 \pm 11.3$ \\
\hline BMI, kg/m2 & $26.7 \pm 7.1$ & $28.9 \pm 3.9$ \\
\hline NYHA class (1:2:3:4) & 1:14:19:2 & 2:3:5:0 \\
\hline \multicolumn{3}{|l|}{ Cause of Pulmonary Hypertension } \\
\hline HРAH (\%) & $2(5)$ & \\
\hline $\mathrm{IPAH}(\%)$ & $14(37)$ & \\
\hline CTD $(\%)$ & $9(24)$ & \\
\hline $\mathrm{CHD}(\%)$ & $8(21)$ & \\
\hline HIV (\%) & $0(0)$ & \\
\hline Portopulmonary (\%) & $4(11)$ & \\
\hline Drugs/toxins/medication (\%) & $0(0)$ & \\
\hline Other $(\%)$ & $1(3)$ & \\
\hline \multicolumn{3}{|l|}{ Type of CTD related PAH } \\
\hline SSc $(\%)$ & $7(78)$ & \\
\hline SLE (\%) & $2(22)$ & \\
\hline Sjogren (\%) & $0(0)$ & \\
\hline \multicolumn{3}{|l|}{ PAH specific drugs } \\
\hline PDE-5 inhibitor (\%) & 33 & 4 \\
\hline ERA $(\%)$ & 33 & 8 \\
\hline Riociguat (\%) & 1 & 1 \\
\hline Prostacycline receptor agonist (Selexipag, \%) & 5 & 0 \\
\hline Prostacycline (IV) $(\%)$ & 6 & 0 \\
\hline Treprostenil (IV/SC) (\%) & 2 & 0 \\
\hline \multicolumn{3}{|l|}{ Drug combination therapy } \\
\hline Mono therapy (\%) & 5 & 3 \\
\hline Duo therapy $(\%)$ & 21 & 5 \\
\hline Triple therapy (\%) & 11 & 0 \\
\hline
\end{tabular}


Table 3.2 (continued)

\begin{tabular}{|l|c|c|}
\hline 6MWD, m & & \\
Baseline & $374 \pm 140$ & $346 \pm 99$ \\
18 months follow up & $445 \pm 142$ & $420 \pm 121$ \\
Echocardiography, RVSP in mmHg & & \\
Baseline & $76.9 \pm 18.6$ & $61.5 \pm 17.5 *$ \\
18 months follow up & $62.7 \pm 22.6$ & $48.1 \pm 17.9$ \\
R-catheterisation (baseline) & $51.3 \pm 13.8$ & $38.9 \pm 13.5 *$ \\
Mean PAP, mmHg & $9.6 \pm 4.9$ & $5.1 \pm 2.6 *$ \\
PVR, WU & $2.6 \pm 0.7$ & $2.7 \pm 0.2$ \\
CI & & \\
\hline
\end{tabular}

$\left({ }^{*}: \mathrm{p}<0.05,{ }^{* *}: \mathrm{p}<0.01\right)$ compared to the PAH group

\subsubsection{Prevalence of abnormal micronutrient and mineral blood concentrations}

The percentage of patients with levels below the reference value for the micronutrients analysed in both patient groups with the diagnostic subclasses are shown in tables 3.3 and 3.4. Levels of micronutrients and nutrient-related factors in all newly diagnosed and treated patients can be found in appendix I.

\subsubsection{PAH patients}

In the newly diagnosed patients with $\mathrm{PAH}, 42 \%$ can be classified as deficient in $25(\mathrm{OH}) \mathrm{D}(60 \%$ of the males and $36 \%$ of the females). Moreover, $21 \%$ of the newly diagnosed $\mathrm{PAH}$ patients has a too low circulating concentration of magnesium.

In the group under treatment for 1.5 years, $68 \%$ of the patients with $\mathrm{PAH}$ has $25(\mathrm{OH}) \mathrm{D}$ levels below normal (79\% of the males and $63 \%$ of the females) and $16 \%$ have a too low circulating concentration of phosphate.

\subsubsection{CTEPH patients}

In newly diagnosed patients with $\mathrm{CTEPH}, 71 \%$ of the patients has $25(\mathrm{OH}) \mathrm{D}$ levels below the reference value ( $63 \%$ of the males and $83 \%$ of the females). Moreover, $14 \%$ show a low circulating concentration of calcium and $7 \%$ have a low circulating concentration of magnesium. 
Of the CTEPH patients after 1.5 years of treatment, $70 \%$ has a too low $25(\mathrm{OH}) \mathrm{D}$ level ( $100 \%$ of the males and $57 \%$ of the females), $20 \%$ has a low circulating concentration of phosphate and $10 \%$ show a low circulating concentration of magnesium.

\subsubsection{Newly diagnosed versus treated patients}

Of all newly diagnosed patients in our study, 15\% has a low circulating concentration of calcium, another $15 \%$ has a low circulating concentration of magnesium and $55 \%$ of the patients show a low $25(\mathrm{OH}) \mathrm{D}$ concentration. Of all treated patients, $69 \%$ show too low $25(\mathrm{OH}) \mathrm{D}$ levels. Furthermore, a total of $17 \%$ of the treated patients have a too low circulating concentration of phosphate.

\subsubsection{Female patients}

Of all female newly diagnosed patients in our study, $50 \%$ has too low $25(\mathrm{OH}) \mathrm{D}$ levels, $20 \%$ has a low circulating concentration of magnesium and $15 \%$ has a low circulating concentration of calcium.

Of all female patients in the treated group, $61 \%$ has a too low $25(\mathrm{OH}) \mathrm{D}$ level and $13 \%$ has a too low circulating concentration of phosphate.

\subsubsection{Male patients}

A total of $62 \%$ of the newly diagnosed males has a low $25(\mathrm{OH}) \mathrm{D}$ concentration, $16 \%$ has a low circulating concentration of calcium and $8 \%$ has a low circulating concentration of magnesium.

After 1.5 years of treatment, $82 \%$ of the males have a too low $25(\mathrm{OH}) \mathrm{D}$ level and $24 \%$ has a low circulating concentration of phosphate.

\subsubsection{Summary}

All in all, a large part of the patients in our study are found to be deficient in 25$(\mathrm{OH})$-vitamin $\mathrm{D}$, both at diagnosis as well as after 1.5 years of treatment. At diagnosis, vitamin D deficiency is more prevalent in CTEPH patients than in $\mathrm{PAH}$ patients. In the treated group, the prevalence of vitamin D deficiency is similar in both PAH and CTEPH patients. Furthermore, low circulating concentrations for magnesium, calcium and phosphate are demonstrated. 
Table 3.3: Percentage of patients below the reference value for micronutrients in newly diagnosed and treated patients, split in gender and disease classification.

\begin{tabular}{|c|c|c|c|c|c|c|c|c|c|c|}
\hline & \multicolumn{5}{|c|}{ Newly diagnosed } & \multicolumn{5}{|c|}{ Treated } \\
\hline & $\begin{array}{c}\text { Total } \\
(\mathrm{n}=33)\end{array}$ & $\begin{array}{l}\text { Female } \\
(\mathrm{n}=20)\end{array}$ & $\begin{array}{c}\text { Male } \\
(\mathrm{n}=13)\end{array}$ & $\begin{array}{c}\text { PAH } \\
(\mathrm{n}=19)\end{array}$ & $\begin{array}{l}\text { СТЕРН } \\
(\mathrm{n}=14)\end{array}$ & $\begin{array}{c}\text { Total } \\
(\mathrm{n}=48)\end{array}$ & $\begin{array}{l}\text { Female } \\
(\mathrm{n}=31)\end{array}$ & $\begin{array}{c}\text { Male } \\
(\mathrm{n}=17)\end{array}$ & $\begin{array}{c}\text { PAH } \\
(\mathrm{n}=38)\end{array}$ & $\begin{array}{l}\text { CTEPH } \\
(\mathrm{n}=10)\end{array}$ \\
\hline Iron $(<10 \mu \mathrm{mol} / \mathrm{l})$ & $33 \%$ & $40 \%$ & $23 \%$ & $42 \%$ & $21 \%$ & $27 \%$ & $36 \%$ & $12 \%$ & $29 \%$ & $20 \%$ \\
\hline Magnesium $(<0.7 \mathrm{mmol} / \mathrm{l})$ & $15 \%$ & $20 \%$ & $8 \%$ & $21 \%$ & $7 \%$ & $4 \%$ & $3 \%$ & $6 \%$ & $3 \%$ & $10 \%$ \\
\hline Calcium $(<2.2 \mathrm{mmol} / \mathrm{l})$ & $15 \%$ & $15 \%$ & $15 \%$ & $16 \%$ & $14 \%$ & $2 \%$ & $0 \%$ & $6 \%$ & $3 \%$ & $0 \%$ \\
\hline Phosphate $(<0.8 \mathrm{mmol} / \mathrm{l})$ & $0 \%$ & $0 \%$ & $0 \%$ & $0 \%$ & $0 \%$ & $17 \%$ & $13 \%$ & $24 \%$ & $16 \%$ & $20 \%$ \\
\hline Vitamin B11 $(<5 \mathrm{nmol} / \mathrm{l})$ & $0 \%$ & $0 \%$ & $0 \%$ & $0 \%$ & $0 \%$ & $0 \%$ & $0 \%$ & $0 \%$ & $0 \%$ & $0 \%$ \\
\hline Vitamin B12 $(<145 \mathrm{pmol} / \mathrm{l})$ & $3 \%$ & $5 \%$ & $0 \%$ & $5 \%$ & $0 \%$ & $4 \%$ & $7 \%$ & $0 \%$ & $5 \%$ & $0 \%$ \\
\hline $25(\mathrm{OH}) \mathrm{D}(<50 \mathrm{nmol} / \mathrm{l})$ & $55 \%$ & $50 \%$ & $62 \%$ & $42 \%$ & $71 \%$ & $69 \%$ & $61 \%$ & $82 \%$ & $68 \%$ & $70 \%$ \\
\hline
\end{tabular}


Table 3.4: Percentage of patients below the reference value for micronutrients in newly diagnosed and treated patients in gender subgroups of PAH and CTEPH patients.

\begin{tabular}{|c|c|c|c|c|c|c|c|c|}
\hline & \multicolumn{4}{|c|}{ Newly diagnosed } & \multicolumn{4}{|c|}{ Treated } \\
\hline & $\begin{array}{l}\text { PAH } \\
\text { male } \\
(n=5)\end{array}$ & $\begin{array}{c}\text { PAH } \\
\text { female } \\
(n=14)\end{array}$ & $\begin{array}{c}\text { CTEPH } \\
\text { male } \\
(n=8)\end{array}$ & $\begin{array}{c}\text { CTEPH } \\
\text { female } \\
(n=6)\end{array}$ & $\begin{array}{c}\text { PAH } \\
\text { male } \\
(n=14)\end{array}$ & $\begin{array}{c}\text { PAH } \\
\text { female } \\
(n=24)\end{array}$ & $\begin{array}{c}\text { CTEPH } \\
\text { male } \\
(n=3)\end{array}$ & $\begin{array}{c}\text { CTEPH } \\
\text { female } \\
(n=7)\end{array}$ \\
\hline Iron $(<10 \mu \mathrm{mol} / \mathrm{l})$ & $20 \%$ & $50 \%$ & $25 \%$ & $17 \%$ & $14 \%$ & $38 \%$ & $0 \%$ & $29 \%$ \\
\hline Magnesium $(<0.7 \mathrm{mmol} / \mathrm{l})$ & $0 \%$ & $29 \%$ & $13 \%$ & $0 \%$ & 0 & $4 \%$ & $33 \%$ & $0 \%$ \\
\hline Calcium $(<2.2 \mathrm{mmol} / \mathrm{l})$ & $20 \%$ & $14 \%$ & $13 \%$ & $17 \%$ & $7 \%$ & $0 \%$ & $0 \%$ & $0 \%$ \\
\hline Phosphate $(<0.8 \mathrm{mmol} / \mathrm{l})$ & $0 \%$ & $0 \%$ & $0 \%$ & $0 \%$ & $29 \%$ & $14 \%$ & $0 \%$ & $29 \%$ \\
\hline Vitamin B11 $(<5 \mathrm{nmol} / \mathrm{l})$ & $0 \%$ & $0 \%$ & $0 \%$ & $0 \%$ & $0 \%$ & $0 \%$ & $0 \%$ & $0 \%$ \\
\hline Vitamin B12 $(<145 \mathrm{pmol} / \mathrm{l})$ & $0 \%$ & $7 \%$ & $0 \%$ & $0 \%$ & $0 \%$ & $8,3 \%$ & $0 \%$ & $0 \%$ \\
\hline $25(\mathrm{OH}) \mathrm{D}(<50 \mathrm{nmol} / \mathrm{l})$ & $60 \%$ & $36 \%$ & $63 \%$ & $83 \%$ & $79 \%$ & $63 \%$ & $100 \%$ & $57 \%$ \\
\hline
\end{tabular}




\subsubsection{Iron status in newly diagnosed and treated $\mathrm{PH}$ patients}

Table 3.5 presents the values for the analytes specifically related to iron status.

In the newly diagnosed group, $30 \%$ of the females and $8 \%$ of the males have too low $\mathrm{Hb}$ levels and could therefore be classified as having anemia. Thirty three percent of the newly diagnosed patients have a low circulating concentration of plasma iron. Of all the newly diagnosed PAH patients, $42 \%$ has low circulating iron concentrations compared to $21 \%$ of the newly diagnosed CTEPH patients. Thirty one percent of the newly diagnosed males and $40 \%$ of the females have a too low transferrin saturation.

In the group under treatment for 1.5 years, $26 \%$ of the females and $24 \%$ of the males have too low $\mathrm{Hb}$ levels and could therefore be classified as having anemia. In this group, $27 \%$ of the patients has a low circulating concentration of plasma iron. Of all the PAH patients after 1,5 years of treatment, $29 \%$ has a low circulating concentration of iron compared to $20 \%$ of the treated CTEPH patients. A total of $59 \%$ of the treated males and $36 \%$ of the females have a low transferrin saturation. In summary, a considerable amount of the patients are deficient in iron, especially female patients. Iron deficiency is higher in PAH patients than in CTEPH patients, both at time of diagnosis as well as after 1.5 years of treatment. 
Table 3.5: Percentage of patients below the reference value for iron related factors in newly diagnosed and treated patients, split in gender and disease classification.

\begin{tabular}{|c|c|c|c|c|c|c|c|c|c|c|}
\hline & \multicolumn{5}{|c|}{ Newly diagnosed } & \multicolumn{5}{|c|}{ Treated } \\
\hline & $\begin{array}{c}\text { Total } \\
(\mathrm{n}=33)\end{array}$ & $\begin{array}{l}\text { Female } \\
(n=20)\end{array}$ & $\begin{array}{c}\text { Male } \\
(n=13)\end{array}$ & $\begin{array}{c}\text { PAH } \\
(n=19)\end{array}$ & $\begin{array}{l}\text { СТЕРН } \\
(\mathrm{n}=14)\end{array}$ & $\begin{array}{c}\text { Total } \\
(\mathrm{n}=48)\end{array}$ & $\begin{array}{l}\text { Female } \\
(n=31)\end{array}$ & $\begin{array}{c}\text { Male } \\
(\mathrm{n}=17)\end{array}$ & $\begin{array}{c}\text { PAH } \\
(n=38)\end{array}$ & $\begin{array}{l}\text { СТЕРН } \\
(n=10)\end{array}$ \\
\hline $\mathrm{Hb}(\mathrm{F}:<7.5 \mathrm{mmol} / \mathrm{l}, \mathrm{M}:<8.5 \mathrm{mmol} / \mathrm{l})$ & $21 \%$ & $30 \%$ & $8 \%$ & $26 \%$ & $14 \%$ & $25 \%$ & $26 \%$ & $24 \%$ & $24 \%$ & $30 \%$ \\
\hline Iron $(<10 \mathrm{mmol} / \mathrm{l})$ & $33 \%$ & $40 \%$ & $23 \%$ & $42 \%$ & $21 \%$ & $27 \%$ & $36 \%$ & $12 \%$ & $29 \%$ & $20 \%$ \\
\hline Ferritin $(\mathrm{F}:<10 \mu \mathrm{g} / \mathrm{l}, \mathrm{M}:<30 \mu \mathrm{g} / \mathrm{l})$ & $3 \%$ & $0 \%$ & $8 \%$ & $0 \%$ & $7 \%$ & $6 \%$ & $0 \%$ & $18 \%$ & $8 \%$ & $0 \%$ \\
\hline Transferrin sat. (F: $<15 \%, M:<20 \%)$ & $36 \%$ & $40 \%$ & $31 \%$ & $47 \%$ & $21 \%$ & $44 \%$ & $36 \%$ & $59 \%$ & $47 \%$ & $30 \%$ \\
\hline
\end{tabular}




\subsubsection{Correlations between micronutrient deficiencies and clinical outcome}

A significant positive correlation is found between the 6MWD and plasma iron levels and between 6MWD and transferrin saturation in both newly diagnosed and treated patients (all $\mathrm{p}<0.01$ ), see appendix II. Looking at the treated group with a result of the 6MWD below and above the group mean (439 meters), we observe that both iron and transferrin saturation levels are significantly lower in the group with a $6 \mathrm{MWD}$ below the mean compared to the group with a 6MWD above the mean (both $\mathrm{p}<0.05$ ), see figure 3.1A-B. NT-Pro-BNP levels of the group with a $6 \mathrm{MWD}$ below the mean are significantly higher than those of the group with 6MWD above the mean (with means of $104.6 \mathrm{pmol} / 1$ and 22.15 pmol/1, respectively, $\mathrm{p}<0.05$, see appendix II). In the newly diagnosed group, both plasma iron and transferrin saturation levels are also significantly lower in the group with a 6MWD below the mean (both $\mathrm{p}<0.05$ ), see figure 3.1C-D. NT-Pro-BNP levels are not significantly different between the newly diagnosed groups with a 6MWD below the mean compared with the group with a 6MWD above the mean (see appendix II). Other micronutrients tested are not significantly different between groups with a $6 \mathrm{MWD}$ below or above the mean. This is the case in both the newly diagnosed and the treated groups.

With respect to the NYHA class, a trend towards higher iron and higher transferrin saturation levels in the lower NYHA classes (NYHA 1 and 2) versus higher the higher NYHA classes (NYHA 3 and 4) is observed in the newly diagnosed patients, but not in the treated patients (see figure 3.2). The mean plasma iron level in newly diagnosed patients was 18.43 micromol/1 in NYHA class 1 and 2 and 12.41 micromol/1 in NYHA class 3 and 4 ( $\mathrm{p}=0.07)$. The mean transferrin saturation in newly diagnosed patients is $27.3 \%$ in NYHA class 1 and 2 compared to $19.0 \%$ in NYHA class 3 and 4 ( $p=0.09$ ). Levels of the other micronutrients tested are not different between groups with a lower and a higher NYHA classes This is the case in both the newly diagnosed and as well as in the treated group. 
a

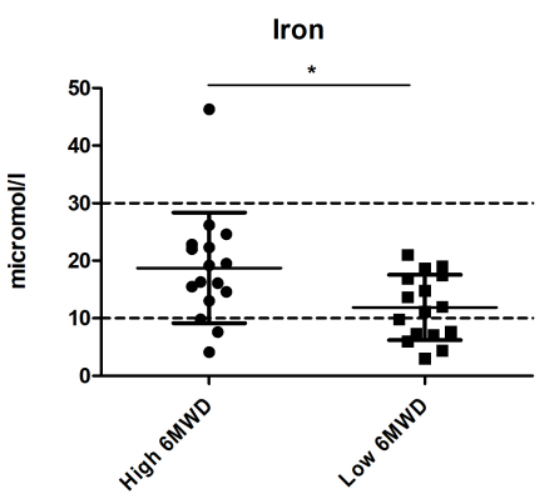

C

Treated

Iron

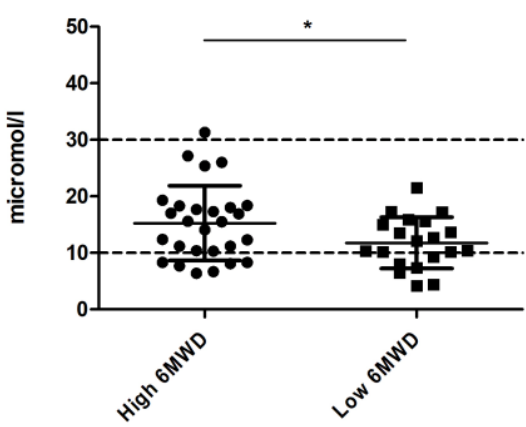

b

Newly diagnosed

Transferrin

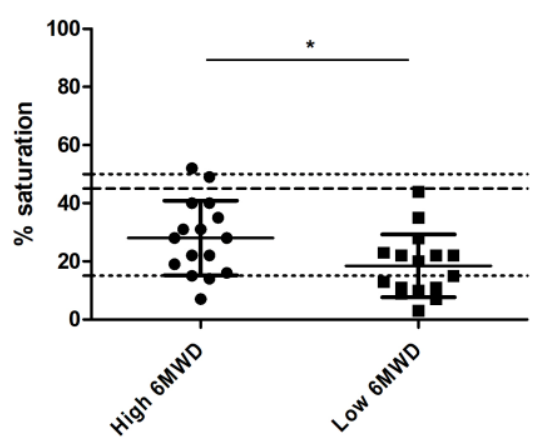

Treated

d

Transferrin

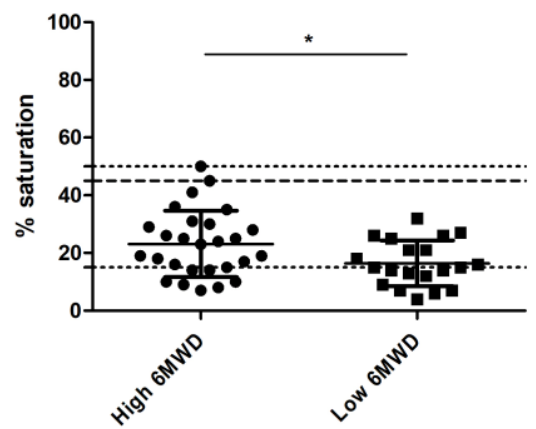

Figure 3.1 Iron levels and transferrin saturation in newly diagnosed and treated patients with higher or lower 6MWD distance than the group mean. $\left(^{*}: \mathrm{p}<0.05\right)$ 
a

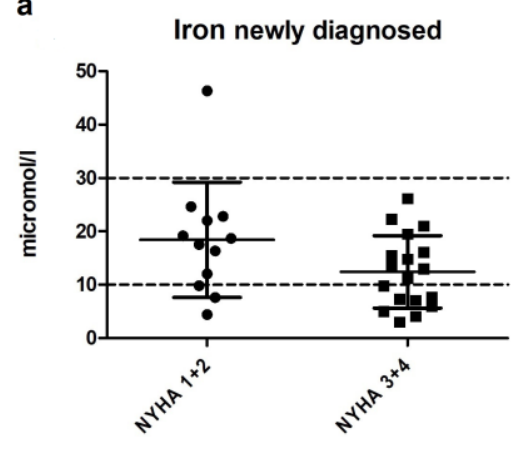

c

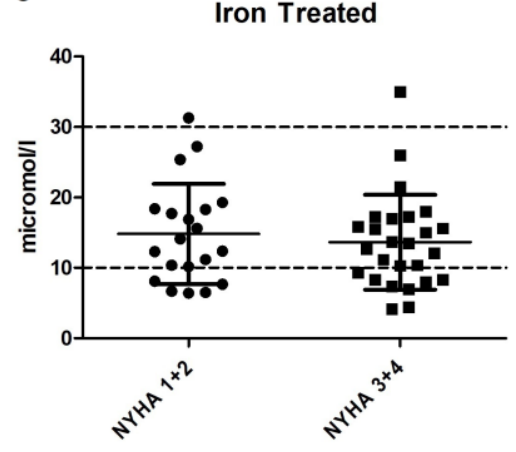

b

Transferrin newly diagnosed

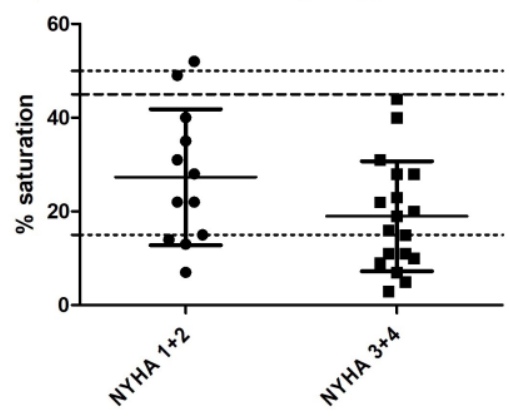

d

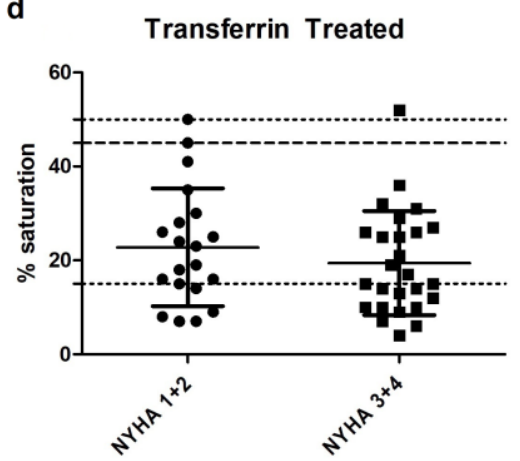

Figure 3.2 Iron levels and transferrin saturation in newly diagnosed and treated patients with NYHA class $1+2$ versus NYHA class $3+4$.

\subsubsection{The relation between iron, hepcidin and inflammation}

Evaluating the results on iron status and inflammation in all patients, it becomes clear that hepcidin levels are higher in newly diagnosed patients compared to treated patients $(p=0.0001$, see figure 3.3A) and ferritin levels as well $(p<0.05$, see figure 3.3B). Albumin levels are significantly lower in the newly diagnosed group compared to the treated group ( $p<0.05$, see figure 3.3C). This suggests that inflammation might play a role. Circulating levels of plasma iron, c-reactive protein (CRP) and $\mathrm{Hb}$ levels are not significantly different between the newly diagnosed and the treated groups (see figure 3.3D-F).

Considering the hepcidin levels of specific patients with low circulating iron concentration, relatively high values are observed in the newly diagnosed group (mean hepcidin level: $121 \mathrm{~g} / \mathrm{l}$, mean iron level: 6.53 micromol/1). At the same time 
these levels are low in patients with low circulating iron concentration in the treated group (mean hepcidin level: $57 \mathrm{~g} / \mathrm{l}$, mean iron level: $7.10 \mathrm{micromol} / \mathrm{l}$ ).
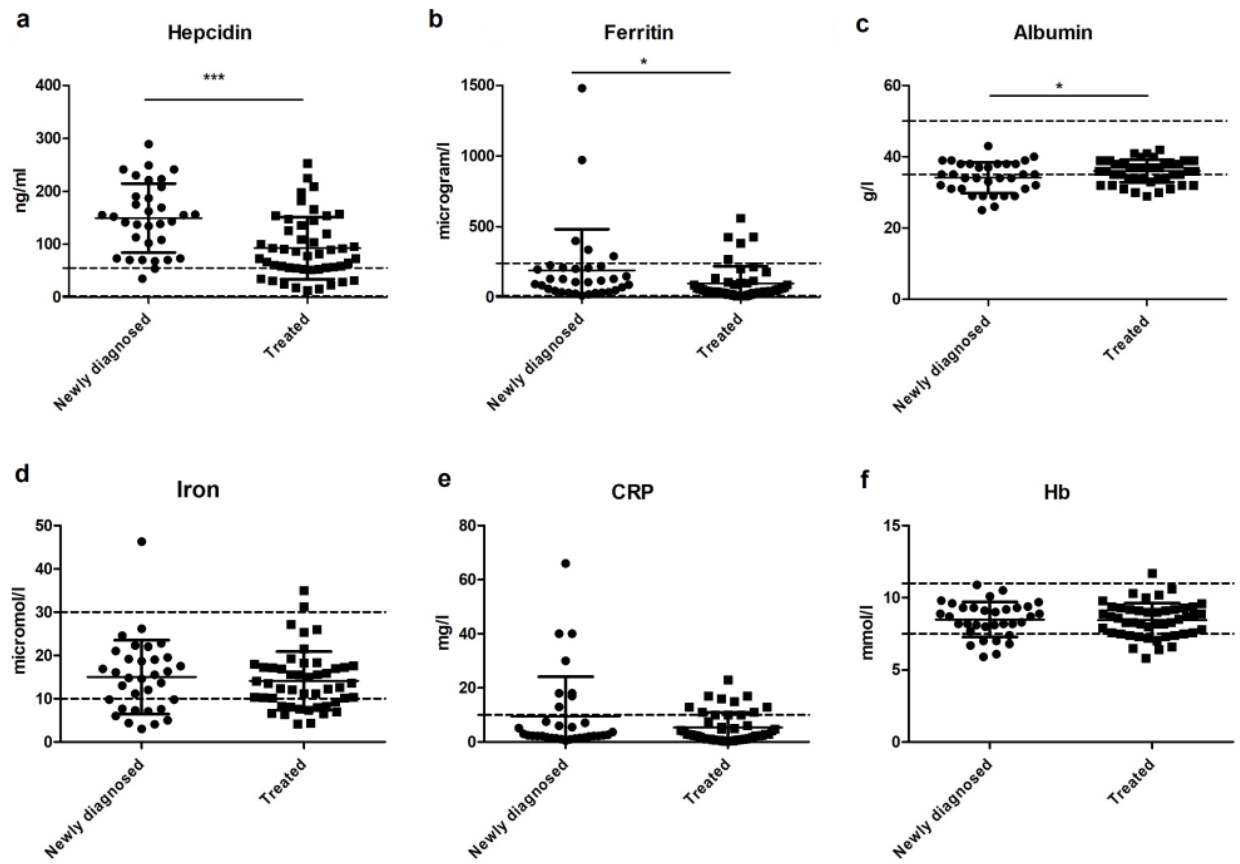

Figure 3.3 Iron, hepcidin and inflammation levels in newly diagnosed and treated patients. $(*: \mathrm{p}<0.05, * * * \mathrm{p}<0.001)$

\subsubsection{Vitamin D binding protein and 25(OH)D}

Levels of DBP are strongly reduced in treated patients compared to newly diagnosed patients (mean values $393.4 \mathrm{mg} / \mathrm{l}$ and $538.6 \mathrm{mg} / \mathrm{l}$, respectively, $\mathrm{p}<0.0001$, see figure $3.4 \mathrm{~A})$. In general, lower DBP levels can lead to lower $25(\mathrm{OH}) \mathrm{D}$ levels measured in plasma. However, in our patients $25(\mathrm{OH}) \mathrm{D}$ levels are not statistically different in treated patients compared to the levels in the newly diagnosed patients (means: 40.0 $\mathrm{nmol} / \mathrm{l}$ and $45.5 \mathrm{nmol} / \mathrm{l}$, respectively), see figure 3.4B. A significant negative correlation is found between $25(\mathrm{OH}) \mathrm{D}$ and CRP levels in newly diagnosed patients $(\mathrm{R}=-0.37, \mathrm{p}<0.05$, see figure $3.4 \mathrm{C})$, but not in treated patients. There is no significant correlation between $25(\mathrm{OH}) \mathrm{D}$ and albumin levels. 
a

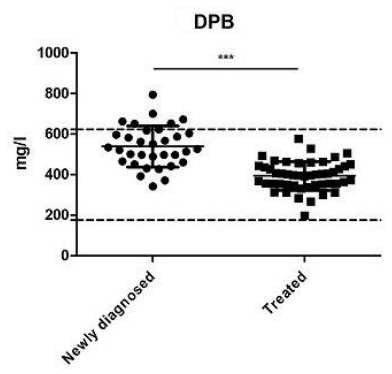

b

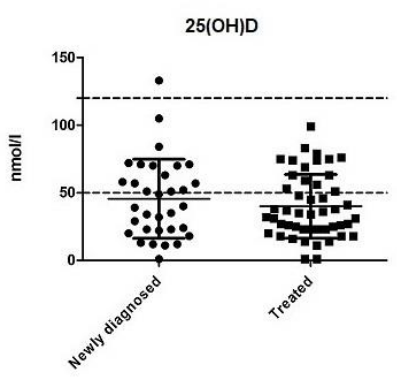

C

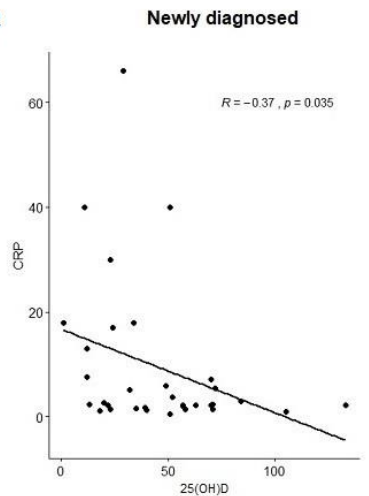

Figure 3.4 Levels of 25(OH)D and vitamin $\mathrm{D}$ binding protein in newly diagnosed and treated patients $\left(^{* * *}: \mathrm{p}<0.0001\right)$.

\subsection{Discussion}

Nutritional status in patients with PAH and CTEPH is an understudied field, although recent papers suggest that nutritional status and micronutrient deficiencies are likely to influence quality of life of these patients via their potential effects on inflammatory status, skeletal muscle functions and other (patho-)physiological processes (Semen \& Bast, 2019; Vinke et al., 2018). Our study is the first showing micronutrient status in both newly diagnosed as well as treated patients with PAH and CTEPH.

Deficiencies in iron and vitamin D are highly prevalent in patients with PAH and CTEPH both at diagnosis and after 1.5 years of conventional treatment. Depending on the diagnostic subclass, iron deficiency occurs in up to $42 \%$ of the patients and vitamin D deficiency in up to $83 \%$ of the patients. $25(\mathrm{OH}) \mathrm{D}$ status even seems to worsen during the disease course. Iron deficiency is more prevalent in female than in male patients and is also more prevalent in patients with PAH than in patients with CTEPH. Other micronutrient deficiencies are observed for magnesium and phosphate with prevalence rates up to $21 \%$. Lower iron levels and transferrin saturation are linked to lower 6MWD in both groups at diagnosis and after 1.5 years of treatment. 


\subsubsection{Iron deficiency}

The present study shows that $42.1 \%$ of the newly diagnosed PAH patients and $21.4 \%$ of the newly diagnosed CTEPH patients has low circulating levels of plasma iron. In the treated group, this is $28.9 \%$ for PAH patients and $20 \%$ for CTEPH patients. Also, in the newly diagnosed group $30 \%$ of the females and $8 \%$ of the males has a too low $\mathrm{Hb}$ level. In the treated group, $25.8 \%$ of the females and $23.5 \%$ of the males has a too low $\mathrm{Hb}$ level, indicating anaemia. An earlier study reported a prevalence of iron deficiency of $43 \%$ in idiopathic PAH patients. Moreover, these authors found that 6MWD was reduced in iron-deficient patients compared to iron-sufficient patients irrespective of the existence of anaemia. (Ruiter et al., 2011). They also demonstrated that intravenous iron therapy in 15 iron-deficient iPAH patients improved exercise endurance capacity, but not 6MWD (Ruiter et al., 2015).

The present study shows that there is a correlation between iron levels, transferrin saturation and 6MWD. Levels of circulating iron and transferrin saturation are significantly lower in patients with a $6 \mathrm{MWD}$ lower than average compared to patients with higher 6MWD.

In addition, our study shows, in agreement with other studies (Ruiter et al., 2011; Soon et al., 2011), that the prevalence of anaemia or iron deficiency is higher in patients with PAH than in patients with CTEPH. This has been suggested to be related to interleukin 6 (IL-6) levels, because IL-6 levels correlated with iron levels in idiopathic PAH patients, but not in CTEPH patients (Soon et al., 2011). Inhibition of dietary iron uptake by the negative regulator of plasma iron levels, hepcidin, might be causally related to this effect. The main iron transporter of the intestine (ferroportin) is inhibited by hepcidin, resulting in a decreased intestinal absorption of iron (Ramakrishnan et al., 2018; Rhodes, Wharton, et al., 2011; Ruiter et al., 2015) . Inflammatory cytokines such as interleukin 1-beta (IL-1b) and IL-6 stimulate hepcidin transcription via different mechanisms, leading to excessive hepcidin production. Interleukin 1-beta might even provide the onset signal, as it induces the transcription of IL-6. This process is also being referred to as inflammation-induced anemia (Kanamori et al., 2017).

In this study, increased inflammation is shown by lower albumin levels in the newly diagnosed group and is also associated with increased hepcidin levels. Hepcidin values found in our study are above the range normally seen in healthy people. However, it should be noted that it is unknown which hepcidin values can be 
expected in chronically ill patients. The physiological challenge for these patients is how to maintain adequate iron stores. Oral supplementation with the recommended daily dose might not be useful when hepcidin levels are high due to underlying inflammation (Ramakrishnan et al., 2018). In this situation, intravenous iron supplementation might be preferable. Taken together, it is currently unknown whether low iron levels can be prevented by supplementation with the normal recommended daily dose of oral iron in combination with anti-inflammatory treatment. Future studies are needed to reveal this.

\subsubsection{Vitamin D deficiency}

Vitamin D deficiency is related to increased inflammation and deregulation of the immune system in several chronic diseases (Sassi et al., 2018). In our patient group, vitamin $\mathrm{D}$ is the most prevalent micronutrient deficiency and this deficiency persists during the disease course. Depending on the therapeutic subclass, vitamin D deficiency occurs in $40-83 \%$ of the patients. In the normal European population, vitamin D deficiency has a prevalence of $40 \%$, when taking vitamin D levels $<50$ $\mathrm{nmol} / \mathrm{l}$ as a cut-off and of $13 \%$ at cut-off levels $<30 \mathrm{nmol} / \mathrm{l}$ (Amrein et al., 2020; Cashman et al., 2016). Another study examined the difference between vitamin D deficiency (25-50 nmol/l) and vitamin D insufficiency (50-75 nmol/l) and found a prevalence of $41 \%$ and $33 \%$, respectively (Casey et al., 2019). A lower DBP level is found in the treated group compared to the newly diagnosed group, although there is no difference in $25(\mathrm{OH}) \mathrm{D}$. It is possible that the treated group has higher levels of free vitamin D. In this study, total vitamin D was measured: free and bound vitamin D. Furthermore, we did not find a correlation between 25(OH)D levels and outcome parameters. Therefore, it remains unclear what exactly is the significance of a vitamin D deficiency in patients with $\mathrm{PH}$.

The question whether supplementation of vitamin D would be clinically useful in patients with $\mathrm{PH}$ is dependent on many factors, such as body fat and lifestyle. It has been found that active elderly who spend much time outdoors have a low risk for vitamin D deficiency (ten Haaf et al., 2019). A large European study found that seasonally adjusted $25(\mathrm{OH}) \mathrm{D}$ concentrations were lower in smokers and higher with increasing ultraviolet $\mathrm{B}$ (UVB) exposure, dietary vitamin $\mathrm{D}$ or oily fish consumption and supplement use with omega-3 fatty acids and vitamin D (Casey et al., 2019). Moreover, having a high BMI or being overweight is associated with lower 
vitamin D status or response to supplementation, which is explained by volumetric dilution and/or sequestration in the adipose tissue (Drincic et al., 2013; Sousa-Santos et al., 2018; Van Dam et al., 2007). There is very limited scientific knowledge on vitamin D supplementation to increase vitamin D status in patients with $\mathrm{PH}$. An uncontrolled longitudinal study supplemented $22 \mathrm{PH}$ patients with 50,000 IU cholecalciferol weekly plus a preparation providing $200 \mathrm{mg}$ magnesium $+8 \mathrm{mg}$ zinc + $400 \mathrm{IU}$ vitamin D daily for 3 months. Serum 25(OH)D and 6MWD significantly increased in this study (Mirdamadi \& Moshkdar, 2016). In another study, vitamin D supplementation increased serum vitamin D levels in a rat model of PH (Tanaka et al., 2017). There is however convincing evidence that vitamin D supplementation is effective to increase $25(\mathrm{OH}) \mathrm{D}$ levels to an optimal level in the general population (Amrein et al., 2020). More studies are needed to establish the effect on vitamin D supplementation on its status and clinical outcome in $\mathrm{PH}$ patients. However, there are recommendations for vitamin D supplementation in the healthy general population that can be used for patients with $\mathrm{PH}$ until disease-specific recommendations can be given (Balvers et al., 2015).

\subsubsection{Summary and recommendations}

In summary, the prevalence of vitamin $\mathrm{D}$ and iron deficiency is high in patients with $\mathrm{PAH}$ and CTEPH. Therefore, it is recommended to monitor iron and vitamin D status in these patients. In case of deficiency, supplementation of iron and vitamin D should be considered. For iron, oral supplementation may not be successful in case of underlying inflammation. In these cases, intravenous supplementation might work. The effect of vitamin D supplementation in $\mathrm{PH}$ patients will need further evaluation.

\subsection{Conclusion}

Both iron and vitamin D deficiency were highly prevalent in 2 groups of PAH and CTEPH patients at time of diagnosis and after 1.5 years of conventional treatment. Vitamin D was the most prevalent micronutrient deficiency in these patient groups. There was no difference between the newly diagnosed and the treatment group. Iron deficiency occurred more often in patients with PAH than in patients with CTEPH and more often in females than in males. Moreover, data suggested that low iron levels negatively impacted exercise tolerance. Therefore, this study shows the need 
for monitoring micronutrient status, especially for iron and vitamin D, in PAH and $\mathrm{CTEPH}$ patients. To treat these deficiencies in PAH and CTEPH patients, future studies are needed to investigate the effect of different supplementation strategies both for iron and for vitamin $\mathrm{D}$.

\section{Acknowledgements}

The authors would like to thank P. Chandoesing, L.M. van den Toorn en R.M. Kauling for their clinical work, Jolande Vis for her help with the laboratory analysis, João Paulo for her help with the statistical analysis and Deniz Gungoroglu for her help with the data analysis. 


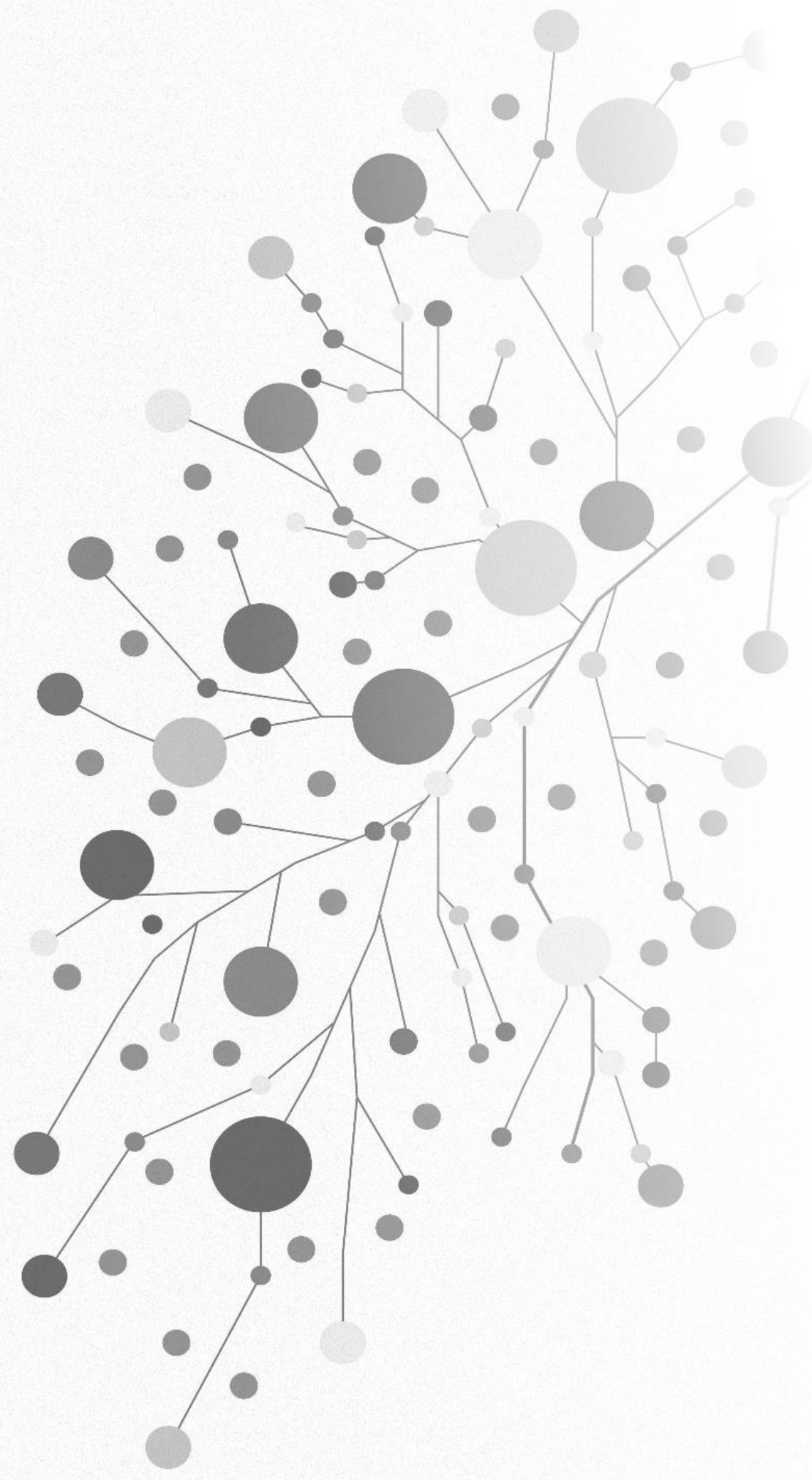


4 The use of proton pump inhibitors may increase symptoms of muscle function loss in patients with chronic illnesses

Paulien Vinke 1,2, Evertine Wesselink ${ }^{3}$, Wout van Orten-Luiten ${ }^{1,4}$, Klaske van Norren ${ }^{1 *}$

\section{Affiliations:}

${ }^{1}$ Nutritional Biology, Division of Human Nutrition and Health, Wageningen University, Stippeneng 4, 6708 WE, Wageningen, the Netherlands

2 Heart Center Leipzig at University of Leipzig, Department of Internal Medicine/Cardiology, Strümpellstraße 39, 04289, Leipzig, Germany

${ }^{3}$ Nutrition and Disease, Division of Human Nutrition and Health, Wageningen University, Stippeneng 4, 6708 WE, Wageningen, the Netherlands

${ }^{4}$ Department of Geriatric Medicine, Gelderse Vallei Hospital, Willy Brandtlaan 10, 6716RP Ede, the Netherlands

Published in: International Journal of Molecular Sciences. 2020; 21(1):323. doi: 10.3390/ijms21010323. 


\begin{abstract}
Long-term use of proton pump inhibitors (PPIs) is common in patients with muscle wasting-related chronic diseases. We explored the hypothesis that the use of PPIs may contribute to a reduction in muscle mass and function in these patients. Literature indicates that a PPI-induced reduction in acidity of the gastrointestinal tract can decrease the absorption of, amongst others, magnesium. Low levels of magnesium are associated with impaired muscle function. This unwanted sideeffect of PPIs on muscle function has been described in different disease backgrounds. Furthermore, magnesium is necessary for activation of vitamin D. Low vitamin $\mathrm{D}$ and magnesium levels together can lead to increased inflammation involved in muscle wasting. In addition, PPI use has been described to alter the microbiota's composition in the gut, which might lead to increased inflammation. However, PPIs are often provided together with nonsteroidal anti-inflammatory drugs (NSAIDs), which are anti-inflammatory. In the presence of obesity, additional mechanisms could further contribute to muscle alterations. In conclusion, use of PPIs has been reported to contribute to muscle function loss. Whether this will add to the risk factor for development of muscle function loss in patients with chronic disease needs further investigation.
\end{abstract}




\subsection{Introduction}

\subsubsection{Cachexia in chronic illness}

There are several definitions of cachexia, but in general, the term includes weight loss, low body mass index, fatigue, imbalance between anabolic and catabolic metabolic pathways, and upregulation of biomarkers of systemic inflammation (Carson et al., 2019; Evans et al., 2008; Fearon et al., 2006; Mak et al., 2011; Muscaritoli et al., 2010). In malnutrition, weight and muscle loss are reversible when adequate amounts of energy and protein are provided. However, in cachexia this is not the case (von Haehling et al., 2016). A consensus definition has been proposed by Evans and colleagues: "Cachexia is defined as a loss of lean tissue mass, involving weight loss greater than $5 \%$ of body weight in 12 months or less in the presence of chronic illness or as a body mass index (BMI) lower than $20 \mathrm{~kg} / \mathrm{m}^{2}$. In addition, three of the following five criteria are also required: decreased muscle strength, fatigue, anorexia, low fat-free mass index, an increase of inflammation markers, anemia, or low serum albumin" (Evans et al., 2008; Society on Sarcopenia Cachexia and Wasting Disorders, 2018). Cachexia is a serious complication that frequently occurs at advanced stage of many chronic illnesses, including cancer, chronic obstructive pulmonary disease (COPD), and cardiovascular disease. Cachexia affects the quality of life and survival of the patients. The prevalence of cachexia ranges between $15 \%$ and $90 \%$ and is dependent on the underlying disease (Scherbakov \& Doehner, 2019; von Haehling et al., 2016). Mortality rates of patients are also disease-dependent and range from $15 \%$ up to $80 \%$ (von Haehling et al., 2016). Muscle mass and muscle function are to a large extent, related. It is only recently that it has become apparent that preservation of only muscle mass without improving muscle function has no effect on quality of life and mortality rates in cancer patients (Garcia, 2017). Apparently, loss of muscle function is contributing to a higher extent to morbidity and mortality than loss of muscle mass. Moreover, for age-related muscle loss, it has been described that loss of muscle function, specifically gait speed, correlates to morbidity and mortality (Tessier et al., 2019). Because most chronically ill patients are older people, this further strengthens the importance of muscle function in the chronically ill patient population. 


\subsubsection{Pathophysiology of cachexia}

Cachexia is a complex and multifactorial disease (Argilés, Busquets, et al., 2014; Laviano et al., 2017). Chronic upregulation of inflammatory mediators, such as interleukin-1 (IL-1), IL-6, tumor necrosis factor- $\alpha$ (TNF- $\alpha$ ), interferon- $\gamma$ (IFN- $\gamma)$, and prostaglandin E2 (PGE2) (Argilés, Busquets, et al., 2014; Noguchi et al., 1996; von Haehling et al., 2017) are an important part of its pathophysiology (von Haehling et al., 2016). These inflammatory mediators, for example, affect appetite-regulating hormones in the hypothalamus, leading to lower food intake (Dwarkasing et al., 2014, 2016). Another important contributor to cachexia is the imbalance between anabolic and catabolic metabolic pathways. This is also partly due to inflammatory processes, but many other mechanisms play important roles as well. As a consequence of the metabolic imbalance, muscle breakdown is increased and muscle synthesis is decreased, leading to lower muscle mass, lower muscle strength, and a sub-optimal body composition (von Haehling et al., 2016). Moreover, muscle function further decreases, because muscle energy metabolism, including mitochondrial functioning, is impaired (Argilés et al., 2015; Argilés, Fontes-Oliveira, et al., 2014; van der Ende et al., 2018). In some cachectic patients, the gut microbiome is altered as well. In cancer cachexia, gut permeability was found to be increased, whereas villi length, crypt dept, cecal content, and tissue weight were reported to be decreased. The inflammatory cytokine IL- 6 was found to be a main driver behind these alterations in the gut (Bindels et al., 2018).

\subsubsection{Sarcopenic obesity in chronic illness}

Sarcopenia in the general sense refers to a condition of reduced muscle mass and functionality. Its underlying causes are diverse and include the process of normal aging. Sarcopenia is an important component of cachexia. The situation in which sarcopenia goes along with obesity is called sarcopenic obesity. This combination is also seen with cachexia and is in cancer independently associated with higher mortality and rate of complications in systemic and surgical cancer treatment (Baracos \& Arribas, 2018; Dolan et al., 2019; Martin et al., 2018; Tan et al., 2009). The prevalence of sarcopenic obesity in advanced solid tumor patient populations is around $9 \%$ (range $2.3 \%-14.6 \%$ ). In this group, even one in four $(24.7 \%$, range $5.9 \%-$ $39.2 \%$ ) of the patients with a body mass index $\geq 30 \mathrm{~kg} / \mathrm{m}^{2}$ is also sarcopenic (Baracos \& Arribas, 2018). Excess visceral adipose tissue seems to be particularly risk 
increasing, whereas subcutaneous adipose tissues appears to be associated with a reduction in overall mortality risk (Martin et al., 2018). This apparent paradox suggests that adipose tissue inflammation, which predominantly occurs in visceral adipose tissue, might play a key role. In cardiovascular diseases and heart failure, this obesity paradox is also frequently reported. A leading theory explains the paradox with the heterogeneity of the obese population, in which different phenotypes occur based on inflammatory characteristics and the relative amount of visceral fat. The three most extreme phenotypes of this hypothesis are formed by the metabolically healthy obesity (MHOB), describing an obese phenotype without metabolic syndrome, metabolically unhealthy obesity (MUO), and the metabolically obese normal weight (MONW) with normal BMI and obesity-related metabolic complications (Vecchié et al., 2018). It seems that with cancer, being obese can be beneficial and reduce mortality risk, as long as the adipose tissue is predominantly subcutaneous, whereas being obese with predominantly visceral (inflammatory) adipose tissue can be harmful and contribute to sarcopenic obesity.

\subsubsection{Adverse effects of long-term use of PPIs}

PPIs are among the most widely used medications worldwide, having high prescription rates, especially for the elderly (Boucherie et al., 2018; Parsons et al., 2012; Zhang et al., 2017). Many patients who suffer from chronic diseases use proton pump inhibitors to reduce the risk of gastric ulceration and ulcer-related bleeding caused by chronic use of nonsteroidal anti-inflammatory drugs (NSAIDs) (Benmassaoud et al., 2016; Kinoshita et al., 2018; Scarpignato et al., 2016). PPIs decrease the acidity in the stomach by inhibition of the $\mathrm{H}^{+} / \mathrm{K}^{+}$-ATPase enzyme in the parietal cells of the gastric glands. As a consequence, the $\mathrm{pH}$ of the proximal intestine is increased (Kinoshita et al., 2018; Shin \& Sachs, 2008), which can affect the gut microbiome (Hojo et al., 2018; Imhann et al., 2016). The use of PPIs has also been associated with a decreased absorption of vitamin $B_{12}$, magnesium, calcium, iron, vitamin $C, \beta$-carotene, and zinc, which is at least in part a direct consequence of their reduced release from the food matrix and (or) effects on the absorption process (Freedberg et al., 2017; Gröber et al., 2020; Kinoshita et al., 2018; Mohn et al., 2018; Sheen \& Triadafilopoulos, 2011) At the clinical level, PPI use has been reported as a variable of importance, after gait speed, oxidative stress, and depression, in the prediction of muscle mass and function loss in aging (sarcopenia) (Cernea et al., 
2019). Moreover, it has been reported that long term use of PPIs can result in polymyositis, a type of chronic inflammation in the muscles (Clark \& Strandell, 2006). Another possible, but rare side-effect of PPI use is rhabdomyolysis, a condition in which damaged muscle breaks down rapidly, and the breakdown product can effect kidney function (Capogrosso Sansone et al., 2017; Vaezi et al., 2017). To conclude, although generally considered safe, long-term use of PPIs could ultimately lead to deficiencies in several vitamins and minerals (Freedberg et al., 2017; Igaz et al., 2018; Mohn et al., 2018). Furthermore, the changed pH can affect the gut microbiome (Hojo et al., 2018; Imhann et al., 2016), and finally, it can result in the presence of myopathy, which might affect muscle function and mass (Cernea et al., 2019).

\subsubsection{Aim and scope}

The adverse effects of long term PPI use can possibly impact the development of muscle mass and function loss (van Dijk et al., 2018) and influence the risk of developing cachexia in the presence or absence of obesity (Bindels et al., 2018; Varian et al., 2016). The aim of this paper is to elaborate on the possible effects of PPI use on muscle function and mass in individuals prone to loss of muscle mass and function, such as chronically ill patients. We focus on the possible effects of PPI use on magnesium levels and vitamin D metabolism, which may impact inflammatory processes in the body that are relevant in the development of muscle mass and function loss in chronic illnesses (Vinke et al., 2018) and on alterations in the gut microbiota (Argilés, Busquets, et al., 2014). Next to that, we evaluate the impact of these deficiencies in a subtype of cachexia patients: the sarcopenic obese patients, because the presence of obesity might further enhance the impact of a reduction in these micronutrient levels. Additionally, we discuss the relation between PPI use, micronutrient status, and muscle function

Proton pump inhibitors can influence the levels of certain micronutrients, such as magnesium, which in turn can influence vitamin D status. Changes in the levels of these micronutrients can enhance the development of chronic low-grade inflammation in chronic disease. 


\subsection{Relation between PPI use, micronutrient status and muscle function}

\subsubsection{PPI use is associated with low magnesium status}

Magnesium functions as a second messenger molecule in many inflammatory processes (Shahi et al., 2019) and is essential in a few hundred enzymatic reactions (de Baaij et al., 2015; Shahi et al., 2019). A normal range of serum magnesium levels is $0.7-1.07 \mathrm{mmol} / \mathrm{L}$ (or $1.7-2.6 \mathrm{mg} / \mathrm{dL}$ ). Magnesium is mostly stored in the bone, muscles, and organs, but lower quantities can be found in the extracellular fluid (de Baaij et al., 2015; Shahi et al., 2019). Average magnesium ingestion from most diets is about $300 \mathrm{mg} /$ day of which $100 \mathrm{mg}$ /day is actually absorbed by the intestine (de Baaij et al., 2015; Shahi et al., 2019). Absorption occurs by a paracellular passive pathway and by active transcellular pathways via the receptors transient receptors potential melastatin 6 and 7 (TRPM6 and TRPM7) (Shahi et al., 2019). Epidemiological studies have shown that low levels of magnesium are associated with several chronic illnesses, such as diabetes and cardiovascular disease (de Baaij et al., 2015; Shahi et al., 2019).

Hypomagnesemia (serum magnesium below $0.7 \mathrm{mmol} / \mathrm{L}$ ) is a known side-effect of PPI use (Freedberg et al., 2017; Gröber et al., 2020; Kinoshita et al., 2018; Mohn et al., 2018; Sheen \& Triadafilopoulos, 2011). PPIs may decrease intestinal magnesium absorption by interfering with passive transport in the ileum and with active transport in the large intestine (de Baaij et al., 2015; Thongon \& Krishnamra, 2012; Toh et al., 2015; William \& Danziger, 2016). The activity of TRMP6 is regulated by intracellular magnesium along with $\mathrm{pH}$, where a lower $\mathrm{pH}$ increases TRMP6 activity. It is hypothesized that PPI use could potentially decrease TRPM6 activity, resulting in decreased magnesium absorption (de Baaij et al., 2015; William \& Danziger, 2016). The prevalence of hypomagnesemia in patients using PPI has been reported to range of 11\%-55\% (Park et al., 2014; van Orten-Luiten et al., 2018). In two recent meta-analyses of nine observational studies with a total of 115,455 and 109,798 patients, pooled relative risks of hypomagnesemia in patients with PPI use were 1.78 (95\% CI 1.08-2.92) (Park et al., 2014) and 1.43 (95\% CI 1.08-1.88) (Cheungpasitporn et al., 2015), respectively. It should be noted that data of six out of a total of 12 original studies were used in both meta-analyses (Cheungpasitporn et al., 2015; Park et al., 2014). In a study in hospitalized elderly using PPI, a hazard risk of 1.80 (95\% CI 1.20-2.72) was observed (van Orten-Luiten et al., 2018). Although results of 
observational studies showed an increased risk of hypomagnesemia in PPI users, the mechanisms by which PPIs induce hypomagnesemia are still being investigated.

\subsubsection{Low magnesium levels are related to decreased muscle function}

Epidemiological data show that serum magnesium levels are independently correlated with muscle performance in older individuals (Dominguez et al., 2006). In a cross-sectional study with adult women (18-79 years), a higher magnesium intake was associated with better indices of skeletal muscle mass and some but not all measures of muscle strength. The association was higher for younger age groups than for older age groups (Welch et al., 2016). Recently, it was also shown that magnesium intake is associated with the prevalence of sarcopenia (ter Borg et al., 2016; Verlaan et al., 2017).

That magnesium supplementation can improve physical performance in elderly women in a weekly exercise program, was shown in a randomised controlled trial (Veronese et al., 2014). Magnesium is thought to help with muscle relaxation and so improve muscle function (van Dronkelaar et al., 2018). Magnesium plays a role in the muscle as a physiological antagonist of calcium, which is also responsible for muscle contraction. Muscle cramps and muscle weakness are indeed often seen in individuals with hypomagnesemia (de Baaij et al., 2015). Systematic reviews have not shown any reduction in muscle cramps after magnesium supplementation in the general public (Garrison et al., 2012), although it might be beneficial during pregnancy (Young \& Jewell, 2002). Because the studies were limited by a small patient population, more research is needed to study the effect of magnesium supplementation on muscle cramps in specific populations.

Next to the effects on muscle function, low magnesium status can also stimulate muscle breakdown. In observational studies, magnesium intake was found to be inversely related to cachexia-associated inflammatory cytokines (Argilés et al., 2019) such as CRP, TNF $\alpha$, and IL-6, in a dose-dependent manner (Galland, 2010). At present, more evidence is accumulating that low magnesium levels can contribute to a state of chronic low-grade inflammation in the body (Galland, 2010; Mazur et al., 2007; Rayssiguier et al., 2010; Shahi et al., 2019). Because magnesium acts as a natural calcium antagonist in a number of processes, the inflammatory response is probably a result of changes in intracellular calcium concentrations by opening of calcium channels and activation of the $N$-methyl-D-aspartate (NMDA) receptor. 
Other possible mechanisms behind its effects on inflammation include activation of phagocytic cells; release of neurotransmitters, such as substance P; ROS production; and activation of NF-kB. Magnesium deficiency can also induce a systemic stress response through neuroendocrinological pathways (Mazur et al., 2007; Shahi et al., 2019).

\subsubsection{Magnesium and vitamin $\mathrm{D}$ deficiency, together, can lead to increased inflammation affecting the muscle}

Next to direct effects of magnesium on inflammation, low magnesium levels may also indirectly increase levels of inflammatory cytokines by inhibition of the production of the active form of vitamin $\mathrm{D}, 1,25(\mathrm{OH})_{2} \mathrm{D}_{3}$, since magnesium plays a key role in vitamin D metabolism (Uwitonze \& Razzaque, 2018). Vitamin D is derived from the production of vitamin D3 in the skin, via a non-enzymatic two-step process induced by UVB radiation and heat, or otherwise obtained from the diet (Bikle, 2014). In the blood, pre-vitamin D is transported by vitamin D binding protein (VDBP). The production of VDBP is magnesium-dependent; therefore, low magnesium levels may lead to lower levels of circulating VDBP, resulting in less transportation of vitamin D to the liver and kidney (Bikle, 2014). In the liver, vitamin D is converted by 25-hydroxylase to 25-hydroxy vitamin D3 $\left(25(\mathrm{OH}) \mathrm{D}_{3}\right)$. Finally, $25(\mathrm{OH}) \mathrm{D}_{3}$ is converted to its active form $1,25(\mathrm{OH})_{2} \mathrm{D}_{3}$ by $1-\alpha$ hydroxylase in the kidney (Bikle, 2014). Both enzymes are magnesium-dependent (Risco \& Traba, 1992). So lower magnesium levels may lead to hampered production of the active form of vitamin D.

Vitamin D plays a crucial role in many physiological functions, including bone and muscle metabolism (DeLuca, 2004). Associations between low vitamin D levels and muscle metabolism disorders have been reported during aging and disease, leading to loss of muscle mass and function (van Dijk et al., 2018). This effect is potentially mediated by the role of vitamin $\mathrm{D}$ in inflammation. The active form of vitamin $\mathrm{D}$, $1,25(\mathrm{OH})_{2} \mathrm{D}_{3}$, also has anti-inflammatory effects (Gonçalves De Carvalho \& Ribeiro, 2017; Guillot et al., 2010). Two main mechanisms by which 1,25(OH) $\mathrm{D}_{3}$ exerts antiinflammatory responses have been suggested. First, $1,25(\mathrm{OH})_{2} \mathrm{D}_{3}$ may inhibit nuclear

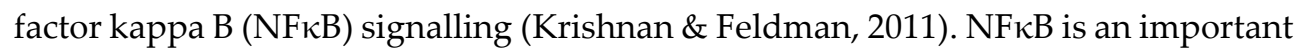
transcription factor involved in the regulation of inflammation. Activation results in the production of pro-inflammatory cytokines (Karin \& Lin, 2002). Second, 
$1,25(\mathrm{OH})_{2} \mathrm{D}_{3}$ may suppress $\mathrm{p} 38$ stress kinase signalling through the upregulation of mitogen-activated protein kinase phosphate 5 , resulting in an inhibition of proinflammatory cytokine production, such as interleukin 6 (IL-6) (Krishnan \& Feldman, 2011). Inflammatory cytokines, especially IL-6, are an important driver of muscle wasting in chronic disease that leads to cachexia.

\subsection{PPI use and the gut microbiota in relation with cachexia}

\subsubsection{The gut microbiota is altered by PPI use}

Proton pump inhibitors elevate the $\mathrm{pH}$ of the stomach and upper gut, which enables more bacteria, including those with pathogenic properties, to survive gastric passage and enter the gut. A study confirmed this by showing that bacteria from the oral cavity are more abundant in the gut microbiomes of PPI users (Imhann et al., 2016). The authors also found that PPI use is associated with a decrease in bacterial richness and considerable changes in the gut microbiome, including significantly increased levels of the class Gammaproteobacteria, the family Enterobacteriaceae and the genus Enterococcus. Increased levels of these bacteria are associated with Clostridium difficile infections in both humans and animal models (Imhann et al., 2016). Several other studies also show that PPI use is associated with increased risk of enteric infections (Bouwknegt et al., 2014; Janarthanan et al., 2012; Leonard et al., 2007; McDonald et al., 2015). These shifts in composition of the microbiota may have immunological consequences, including an elevated pro-inflammatory status.

Interestingly, mice on a magnesium-deficient diet were found to develop a microbiota composition that is considered less favorable for health and an attenuated gut barrier function compared to mice on a magnesium sufficient diet (Pachikian et al., 2010). This supports a link between low magnesium levels and a less healthy gut microbiota that affect inflammation and metabolic disorders (Pachikian et al., 2010).

Moreover, a small human study suggests that consumption of inulin can improve blood magnesium concentrations in proton pump inhibitor-induced hypomagnesaemia. The explanation of the effect may lie in a combination of changed $\mathrm{pH}$ levels of the colon and a change in microbiota (Hess et al., 2016). Interestingly, the study of Winther and colleagues showed (Winther et al., 2015) that a magnesium-deficient diet altered the gut microbiota of mice, and this led to depression-like behavior. The altered gut microbiota also correlated positively with 
IL-6 levels in the hippocampus, suggesting that inflammatory processes in the brain played a role (Winther et al., 2015). As discussed before, similar inflammatory mediators have been shown to affect appetite-regulating hormones in the hypothalamus, leading to lower food intake (Dwarkasing et al., 2014, 2016). What has to be taken into account, however, is that PPIs are often taken together with NSAIDs that will counteract this effect. In view of these findings, crosstalk between the PPI-induced processes leading to inflammation might occur when PPIs are provided in the absence of NSAIDs (figure 4.1), while the effect on muscle function is more likely to occur both in the presence and absence of NSAIDs. This might be the reason why for PPIs side-effects on muscle function are described while effects on muscle wasting are not. There is, however, a knowledge gap for situations in which PPIs are provided in the absence of NSAIDs and for the situation in which the combination treatment of PPIs with NSAIDs is stopped and gut microbiota might be altered, as described in the next paragraph.

\subsubsection{Alterations in gut microbiota can contribute to both muscle wasting and obesity}

An increase in Enterobacteriaceae can be seen in both cachectic patients and in PPIusers, which results in increased LPS production and activation of TLR4, increasing inflammatory status (Garcia, 2017). Moreover, animal data indicate that the PPI omeprazole reduces microbiota diversity. In (Garcia, 2017), microbiota-derived formate levels were increased. Microbiota-derived formate levels have been associated with increased intestinal inflammation. Next to that, the authors showed an association between a low dietary magnesium bioavailability and formate levels. These data indicate that changes in microbiota might occur due to PPI use (Gommers et al., 2019). Next to that, increased TLR4 stimulation by LPS has been reported to increase hypothalamic inflammation, inducing disease-induced anorexia and loss of muscle mass and function (Van Norren et al., 2017; Witkamp \& van Norren, 2018). Bacteria in the gut also produce metabolites and contain structural components that act as signaling molecules to enteroendocrine cells in the mucosa. These cells in turn produce regulatory hormones (e.g., CCK, PYY, GLP-1, and serotonin) in key metabolic processes such as appetite regulation, glucose tolerance, and fat storage in the body (Luttikhold et al., 2013; Martin et al., 2019). Alterations in the gut microbiota can, therefore, impact metabolic disorders such as cachexia, either 
directly, via metabolic deregulation, or indirectly, by contributing to the underlying inflammatory processes of chronic illness. The impact of gut health on cachexia has recently been shown in animal models for cancer cachexia (Bindels et al., 2018). In COPD patients, there are indications of decreased splanchnic perfusion and an impaired barrier function, leading to a reduction in the uptake of nutrients from the gut and contributing to inflammation (Sanders et al., 2016). Moreover, alterations in microbiota also play a key role in the development of obesity. There are many mechanisms involved, such as increased energy uptake from the food due to fermentation of the gut microbiota and influencing appetite regulation and metabolism via signaling molecules (Baothman et al., 2016; Gérard, 2016).

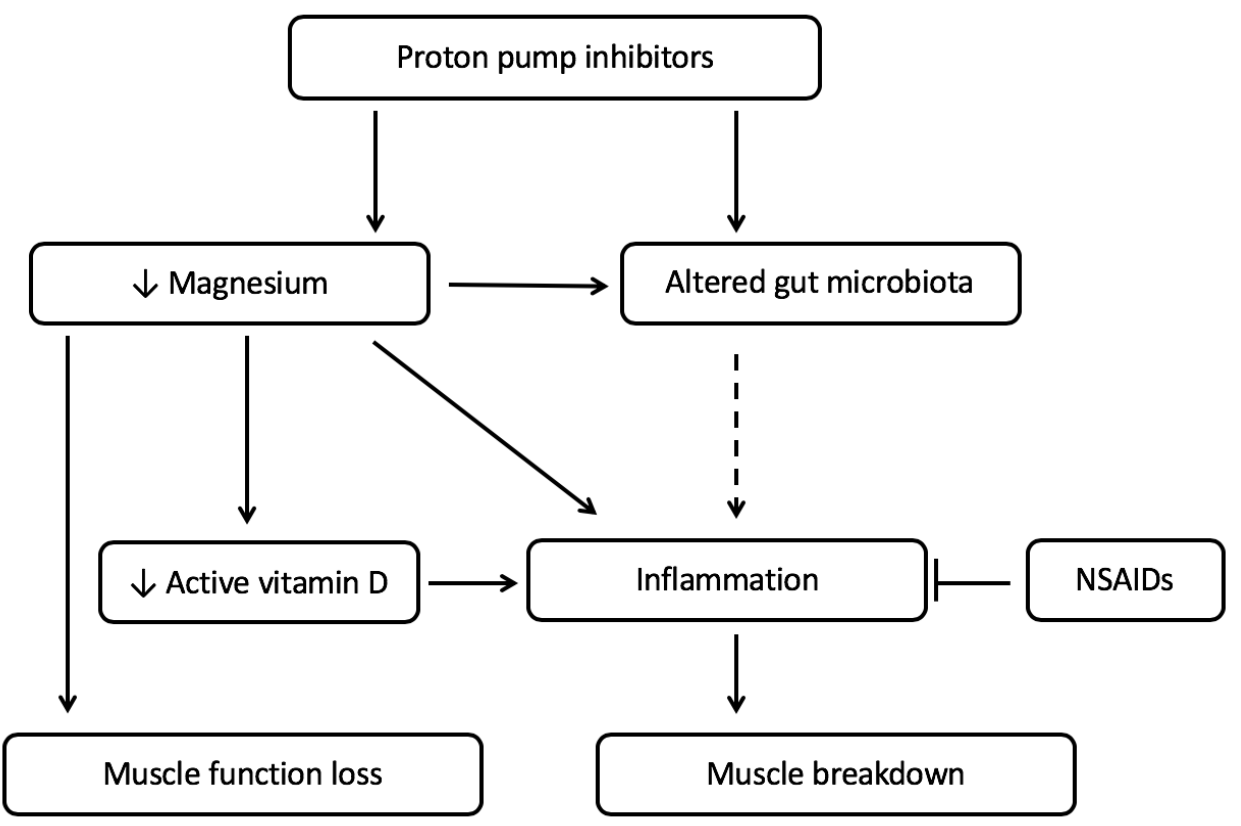

Figure 4.1 The proposed mechanism by which the use of proton pump inhibitors can lead to increased muscle function loss and increased muscle breakdown in cachexia-related chronic diseases. The use of proton pump inhibitors leads to an increase in chronic low-grade inflammation by altering the gut microbiota and decreasing magnesium and vitamin $\mathrm{D}$ levels. Lower magnesium levels lead to muscle function loss and increase inflammation directly and indirectly via vitamin $D$. The increase in inflammation leads to muscle breakdown. When PPIs 
are given together with NSAIDs, it is likely that the effect on inflammation is abandoned. The impact of PPI use on muscle function is likely not affected by the use of NSAIDs.

\subsection{PPI use and muscle wasting in the presence of obesity}

Obesity is associated with magnesium and vitamin D deficiencies; metabolic disturbances, such as insulin resistance; and chronic low-grade inflammation. Given the key role for inflammation in the development of muscle wasting, PPI use might further worsen the condition of an obese patient by increasing muscle wasting via an increase in inflammatory responses.

\subsubsection{Low magnesium levels are often seen in obesity}

Magnesium deficiencies have been reported frequently in obesity (De Leeuw et al., 1992; De Oliveira et al., 2017; Farhanghi et al., 2009; Guerrero-Romero et al., 2016; Günther, 2011; Johansson et al., 2009; Kurpad \& Aeberli, 2012; Morais et al., 2017; Morrell et al., 2012; Mostafavi et al., 2015; Nielsen, 2010; Resnick, 1992). Different causal factors between obesity and hypomagnesemia have been suggested. A relationship between insulin sensitivity and magnesium levels (De Leeuw et al., 1992; Gagnon et al., 2011; Liu et al., 2019; Mostafavi et al., 2015) and an inadequate intake due to a poor quality of the diet (Astrup \& Bügel, 2019) were reported as key factors contributing to the association between obesity and low magnesium levels. This poor nutritional intake can further worsen in the presence of a restricted diet, if this is not supported by nutritional counseling. The dietary guidance for patients with heart failure (HF) (Vest et al., 2019), for example, states that next to the traditional focus on sodium and fluid intake restriction, adequate intake of macroand micronutrients should be monitored and properly counseled as well, because it may influence morbidity and mortality (Vest et al., 2019). The link with insulin sensitivity rather than with obesity further supports the need to look into obese phenotypes, since the visceral obese inflamed phenotype is also related to the presence of insulin resistance. Another important causal factor for low magnesium levels in obesity is the increased blood levels of non-esterified or free fatty acids that directly bind to magnesium and so reduce the free magnesium concentration in the blood (Kurstjens et al., 2019). 


\subsubsection{Low magnesium levels lead to insulin resistance}

In obesity, a chronic, low-grade inflammatory state is involved in the development of insulin resistance (IR), metabolic syndrome, and type II diabetes mellitus (T2DM) (McGill et al., 2008). Currently, more awareness is also rising of the fact that low magnesium levels occur at higher frequency in diabetic patients (Mostafavi et al., 2015). The underlying mechanism has been elucidated to a certain extent. Insulin stimulates magnesium uptake in insulin-sensitive tissues like muscle. Subsequently, the magnesium is used by the tissue to form magnesium-ATP complexes. Lower levels of magnesium-ATP complexes result in insulin resistance, reducing muscle glucose utilization. For the muscle, this implies lower energy status, resulting in increased muscle breakdown. Next to that, magnesium is involved in insulin signaling. Lower intracellular pancreatic $\mathrm{Mg}$ levels result in a reduction of insulin secretion (Mostafavi et al., 2015).

\subsubsection{Vitamin D deficiency is prevalent in obesity}

Systematic reviews and meta-analyses have shown that obese individuals are at a greater risk for vitamin D deficiency (Pereira-Santos et al., 2015; Slusher et al., 2015), although the intake of vitamin $\mathrm{D}$ from food or supplements is not reduced in obese individuals (Slusher et al., 2015). This can be explained by the finding that researchers showed an inverse association between BMI and the response to vitamin D supplementation to increase serum vitamin D levels. It is hypothesized that the increased amount of adipose tissue withdraws more fat-soluble vitamin D in obese individuals compared to non-obese individuals, leading to a decreased bioavailability of vitamin D from cutaneous and dietary sources (Wortsman et al., 2000). Vitamin D deficiencies should be avoided, because adequate vitamin D levels in obese individuals are correlated with an increase in adiponectin. This adipocytokine is involved in regulation of glucose levels and fatty acid breakdown and can so help reduce the metabolic disturbance and the development of insulin resistance as seen in obesity (Stokić et al., 2015).

Moreover, adequate levels of vitamin D act as a suppressor of pro-inflammatory cytokines IL-6 and TNF $\alpha$ (Gao et al., 2013). These cytokines are upregulated in the obese state and contribute to metabolic disturbance (Kahn et al., 2006). A deficiency of vitamin D in obesity might contribute to the increased inflammatory state that is already seen in obesity and that contributes to sarcopenic obesity. 


\subsubsection{PPI use might increase prevalence of micronutrient deficiences and enhance development of sarcopenic obesity}

The presence of obesity might aggravate an effect of PPIs on muscle wasting and lead to the development of sarcopenic obesity. In obesity, the development of insulin resistance and lower levels of magnesium, vitamin D, and adiponectin contribute to chronic low-grade inflammation, the underlying factor for muscle wasting. Adequate levels of both vitamin $\mathrm{D}$ and magnesium might prevent this effect and contribute to a decrease of the inflammatory state and a reduction in metabolic disturbances, such as insulin resistance. Because the use of proton pump inhibitors is likely to increase the severity of these deficiencies, the use of proton pump inhibitors might contribute to an enhancement of the mechanisms involved in the development of sarcopenic obesity (figure 4.2).

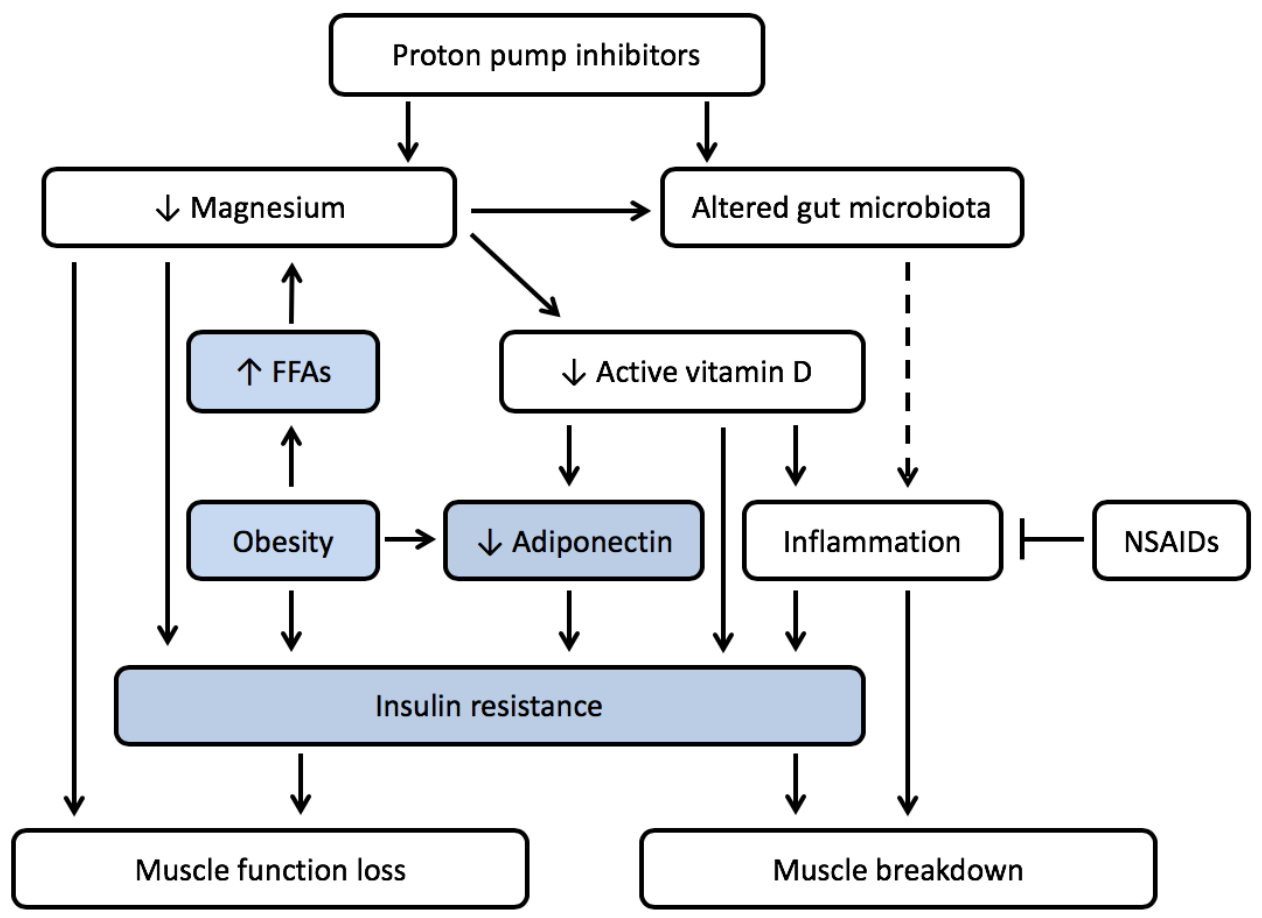

Figure 4.2 The proposed mechanism by which the use of proton pump inhibitors can lead to increased muscle breakdown in the presence of obesity. The use of proton pump inhibitors may lead to an increase in chronic low-grade 
inflammation by altering the gut microbiota and by lowering magnesium and vitamin D levels. Obesity contributes to the presence of low magnesium levels via increased free fatty acids (FFAs) and insulin resistance (directly and via lower adiponectin levels). The increase in inflammation also contributes to insulin resistance. Low magnesium levels and insulin resistance both lead to muscle function loss. The increased inflammation, together with insulin resistance, lead to muscle breakdown. This leads to the development of sarcopenic obesity. When PPIs are given together with nonsteroidal anti-inflammatory drugs (NSAIDs), the (increase in) inflammation is likely diminished, leading to no additional PPIinduced muscle breakdown. The impact of PPI use on muscle function is likely not strongly affected by NSAIDs, and might, therefore, occur both in the presence and absence of PPIs. The obesity-related mechanisms that contribute to muscle function loss and muscle breakdown are highlighted in blue.

\subsection{Discussion}

In this paper, we hypothesized that long term use of proton pump inhibitors contributes to an increase in symptoms of cachexia and sarcopenic obesity in patients with chronic illness. This effect might be mediated by negatively influencing the status of magnesium and by alterations in the gut microbiota. Magnesium deficiency in turn, might negatively impact the level of active vitamin D. A low magnesium status, lower levels of active vitamin D, and a less healthy gut microbiota might all contribute to the state of chronic low-grade inflammation in chronic illness that is a key symptom in the development of muscle wasting in the presence or absence of obesity. Next to that, a less healthy microbiota might also directly contribute to the metabolic deregulation in cachexia and sarcopenic obesity. Figure 4.1 summarizes the hypothesized pathway from PPI-induced changes in magnesium and the microbiota to loss of muscle function in chronic illness-induced (pre-)cachexia. Concerning the PPI-induced changes, the change in $\mathrm{pH}$ induces changes in gut microbiota and receptor-mediated uptake of magnesium. Low magnesium levels lead to decreased muscle function. A reduced level of magnesium also reduces the activation of vitamin $\mathrm{D}$, and alters the gut microbiota as well. Both an altered microbiota and reduced levels of magnesium and active vitamin D can contribute to the inflammation that is already present due to the chronic illness and the presence of (pre-)cachexia. In this way the inflammatory status worsens and may 
lead to increased muscle breakdown. NSAIDs are often prescribed in chronic illnesses and often combined with the use of PPIs to reduce side-effects. When PPIs are given together with NSAIDs, the (increase in) inflammation is likely to be reduced, leading to less muscle breakdown. The impact of PPI use on muscle function loss might, however, not be corrected by NSAIDs. Moreover, the effect on microbiota might remain after termination of the combined NSAID and PPI treatment. Whether PPI use has a negative effect on muscle function and muscle breakdown in the context of NSAID use, therefore, needs further investigation.

Figure 4.2 summarizes the hypothesized pathway from PPIs-induced changes in the presence of obesity that may lead to the development of sarcopenic obesity. Next to the pathways discussed in Figure 4.1, in this case the obese state influences magnesium levels itself via an increase in free fatty acids, contributes to the development of insulin resistance via a decrease in vitamin $\mathrm{D}$ and adiponectin, and leads to the development of muscle wasting or sarcopenic obesity. It is likely that in the presence of visceral adipose tissue-induced inflammation, as is present in sarcopenic obesity, the inflammatory status can be further affected by use of PPIs, leading to accelerated muscle breakdown. This, however, needs further investigation. In view of these findings, possible side effects of PPIs in patients with chronic illnesses susceptible to the development of cachexia, including sarcopenic obesity, merit further study.

The question that remains is whether the benefits of PPI use outweigh the risks in chronically ill patients with risk of muscle mass and function loss. If properly prescribed (Freedberg et al., 2017), it is likely that they do. PPIs contribute extensively to reductions in all kinds of upper gastro-intestinal complications and their prevention if NSAIDs are taken (Freedberg et al., 2017; Lanas-Gimeno et al., 2019). Proton pump inhibitor use has also been reported to be associated with a lower risk of acute exacerbation and mortality in COPD patients with gastroesophageal reflux disease (Su et al., 2018). Moreover, the level of evidence of the risks of PPIs is still low. One should also take into account that the possibility of a relation of PPI with sarcopenia was based on a study with a limited number of subjects $(n=200)$. The paper, however, does not indicate if corrections for confounding factors were made. This sarcopenic population is older, has a higher prevalence of co-morbidities, and has poly-drug use, which might all contribute to 
confounding factors. On the other hand, are the data obtained with a data-driven machine-learning model to characterise factors involved, which are to a certain extend unbiased. Still, causal relationships need to be further investigated.

The mechanistic aspects described in this paper are in part based on animal research. In humans, the relationships between PPI use and magnesium levels or between magnesium and inflammation were investigated only in epidemiological studies or described in case reports (Cabras et al., 2020; Cheungpasitporn et al., 2015; Park et al., 2014). For our hypothesis of PPI use leading to muscle function loss, most support from literature is available. For the effect of PPI on magnesium levels, a recent meta-analysis indicated a 1.4-fold increase on the risk of developing hypomagnesia in PPI-users (Liao et al., 2019). However, authors indicate that heterogeneity in de data reduced the level of evidence, and therefore, more studies are needed to come to a high level conclusive answer. The in Figures 4.1 and 4.2, predicted side effects of PPI use, however, might add to existing muscle function loss and might be prevented by nutritional interventions, which warrants further investigation.

The relationship between PPI use and muscle protein breakdown has not been described in the literature. In practice, PPIs are (most of the time) provided together with NSAIDs, which means that the possible inflammatory effect of PPIs might be superseded by the anti-inflammatory effect of the NSAIDs. There is, however, some data supporting a PPI-induced change in microflora. This indicates that after termination of the combined PPI and NSAID treatment, inflammation might be induced by the still-altered gut microbiota. It is, however, purely hypothetical because it is not possible to establish a direct cause and effect relationship based on the data available.

Magnesium and vitamin D deficiencies and alterations in the gut microbiota have been reported in chronic diseases such as cancer, heart failure, and COPD. In cancer, multiple pathological changes contribute to an altered energy metabolism and anorexia, which both contribute to nutrient deficiencies (Argilés, Busquets, et al., 2014). Gut barrier dysfunction and bacterial translocations are associated with cancer, also in the absence of chemotherapy (Bindels et al., 2018; Klein et al., 2013). Patients with heart failure have a low intake of several micronutrients, such as magnesium, vitamin $\mathrm{D}$, and iron. These micronutrients are important cofactors for normal cardiac metabolism and deficiencies are often seen during progression of the 
disease (Cascino \& Hummel, 2018; Soukoulis et al., 2009). The development of anorexia and malnutrition further contributes to the development of micronutrient deficiencies in patients with advanced heart failure (von Haehling et al., 2017). Moreover, alterations in the gut were reported in those patients: ischemia of the intestinal mucosa may lead to an increased gut permeability and bacterial translocation (Sundaram \& Fang, 2016). In COPD patients, micronutrient deficiencies are also common; in particular, for vitamin D, vitamin B12, and iron (Horadagoda et al., 2017; Schols et al., 2013; 2014).

Another point to consider is that interpretation of the relationship between magnesium levels and health is difficult for two main reasons. First, discussions are ongoing on the current serum magnesium reference interval of $0.70-0.95 \mathrm{mmol} / \mathrm{L}$ (Costello et al., 2016). Second, only $0.8 \%$ of the total magnesium in the body is available in serum (de Baaij et al., 2015), making it questionable whether serum magnesium is a sensitive and specific biomarker of magnesium status (Witkowski et al., 2011). Serum magnesium levels can still be in the normal range, even when body stores of magnesium are depleted. In addition, the exact mechanisms by which PPI induce magnesium deficiency are still being investigated. It is, however, reported frequently that long term PPI use can decrease magnesium levels (Freedberg et al., 2017; Gröber et al., 2020; Mohn et al., 2018; Sheen \& Triadafilopoulos, 2011) and that low magnesium levels are associated with increased risk of chronic illness (Shahi et al., 2019). More insight into the impact of PPI use on magnesium status and a more reliable biomarker of magnesium status is necessary.

To summarize, patients with chronic illness have been described to be susceptible to develop micronutrient deficiencies and an altered gut microbiota. PPI use has been linked to both phenomena, but causal relationships need further investigations (Freedberg et al., 2014; Strand et al., 2017; Wilhelm et al., 2013; Yu et al., 2017). Therefore, we suggest monitoring the micronutrient status in patients on long term use of proton pump inhibitors. In case of a vitamin D deficiency, supplementation of vitamin $\mathrm{D}$ together with magnesium might be considered if magnesium intake or blood levels are low. 


\subsection{Conclusions}

The mechanisms and relationships described in this review underline that use of proton pump inhibitors might add to the symptoms of muscle function loss in patients with chronic illness. There is a need for prospective studies among these patients to investigate whether PPI use increases muscle function loss and inflammation, during and after use of PPIs, with special attention for gut microbiota, and vitamin $\mathrm{D}$ and magnesium status.

\section{Acknowledgements}

We would like to thank Sabien van Exter and Thirsa Brommer for contributing to the literature research of this paper. 


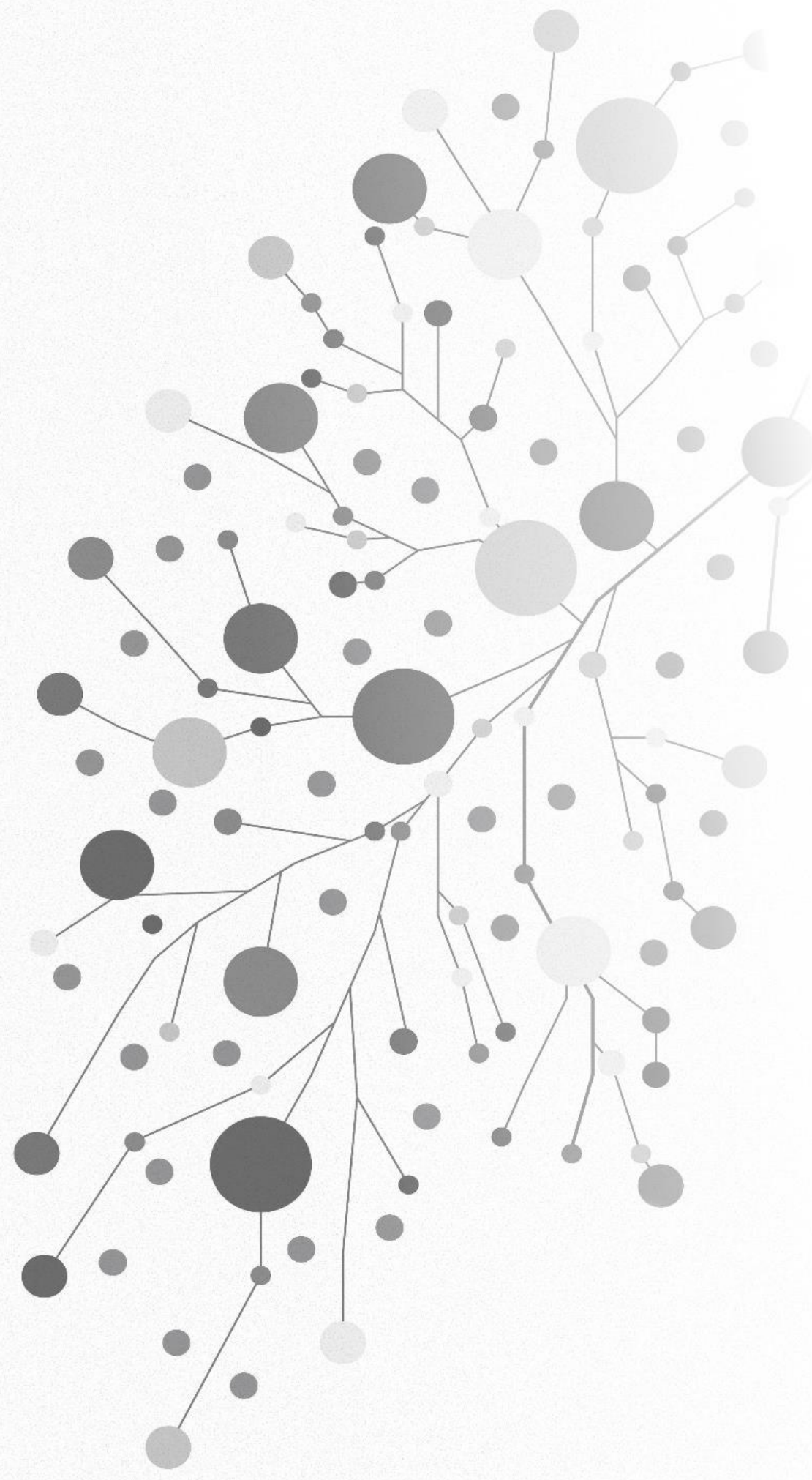


$5 \quad$ Anti-inflammatory nutrition with high protein attenuates cardiac and skeletal muscle alterations in a pulmonary arterial hypertension model

Paulien Vinke 1,2*, T. Scott Bowen 3, Mark. V. Boekschoten 4, Renger F. Witkamp 1, Volker Adams $^{5}$, Klaske van Norren ${ }^{1 \S}$

\section{Affiliations:}

${ }^{1}$ Nutritional Biology, Division of Human Nutrition and Health, Wageningen University, Stippeneng 4, 6708 WE, Wageningen, the Netherlands

${ }^{2}$ University Clinic of Cardiology, Heart Center Leipzig, Strümpellstraße 39, 04289, Leipzig, Germany

${ }^{3}$ School of Biomedical Science, University of Leeds, Clarendon Way LS2 9JT, Leeds, United Kingdom

${ }^{4}$ Nutrition, Metabolism \& Genomics Group, Division of Human Nutrition and Health, Wageningen University, Stippeneng 4, 6708 WE, Wageningen, the Netherlands

${ }^{5}$ Laboratory of molecular and experimental cardiology, Dresden Heart Center, Fetscherstraße 76, 01307, Dresden, Germany

$\S$ contributed equally

Published in: Scientific Reports. 2019; 9(1):10160.

doi: 10.1038/s41598-019-46331-4. 


\begin{abstract}
Pulmonary arterial hypertension $(\mathrm{PAH})$ is characterized by remodeling of the pulmonary arteries and right ventricle (RV) and leading to functional decline of cardiac and skeletal muscle. This study investigated effects of a multi-target nutritional combination with extra protein, leucine, fish oil and oligosaccharides on cardiac and skeletal muscle in a PAH model. PAH was induced in female C57/BL6 mice by weekly injection of monocrotaline (MCT) for 8 weeks. One MCT group and the sham group received a control diet, while another MCT group received the isocaloric nutritional intervention. The results show that, compared to sham, MCT mice showed an increase in heart weight by $7 \%$, RV thickness by $13 \%$ and fibrosis by $60 \%$ (all p<0.05), which were attenuated in MCT+NI mice. Microarray and qRTPCR analysis on the RV confirmed effects on fibrotic pathways. Skeletal muscle fiber cross-sectional area (CSA) of the TA was reduced $(\mathrm{P}<0.05)$ by $22 \%$ in MCT compared to sham mice, but preserved in the MCT+NI group. In the EDL a similar effect was seen. The findings show that nutritional intervention significantly attenuated changes in both cardiac and skeletal muscle in a mouse model of PAH, providing directions for future therapeutic strategies targeting functional decline of both tissues.
\end{abstract}




\subsection{Introduction}

Pulmonary hypertension $(\mathrm{PH})$ is a syndrome that can result from different pathological changes in the pulmonary vasculature. $\mathrm{PH}$ is a progressive disease that is incurable and eventually lethal. An idiopathic or heritable form of $\mathrm{PH}$, called pulmonary arterial hypertension (PAH), is the most severe form. PAH occurs mostly in women. The prevalence of PAH in Europe is estimated to be 15-100 subjects per million, with 1- and 3-year survival rates of $87 \%$ and $67 \%$, respectively (Alami et al., 2016; Galiè, Humbert, et al., 2015). The pathophysiology of PAH involves endothelial dysfunction of the pulmonary vasculature, metabolic shifts in vascular cells, hypertrophy and proliferation of smooth muscle cells, and uncontrolled growth of neointimal, medial, and adventitial layers, leading to thickening and occlusion of the small- and medium-sized pulmonary arteries (de Jesus Perez, 2016). Next to vascular changes, right ventricular (RV) remodeling is a major characteristic of PAH. RV dysfunction is considered to be mainly responsible for disease progression and the symptoms impairing quality of life, such as shortness of breath, exercise intolerance and fatigue (Galiè, Humbert, et al., 2015). Other pathophysiological processes that contribute to exercise intolerance in $\mathrm{PAH}$ include inflammation, dysregulated metabolism, skeletal muscle alterations, and impaired nutritional status (Vinke et al., 2018).

Three known pathways involved in PAH pathophysiology are currently targeted by drugs (Galiè, Humbert, et al., 2015), i.e. the endothelin pathway, the nitric oxide pathway and the prostacyclin pathway (Humbert et al., 2014). Compounds used aim to reduce pulmonary vascular resistance, provide relief of symptoms, and delay disease progression, but are neither disease-modifying nor treating extra-cardiac impairments. This means that patients live longer, but their quality of life is impaired by symptoms of fatigue and exercise intolerance. Therefore, the development of novel treatment options, targeting both the heart as well as other extracardiac organs, such as skeletal muscle, is of great clinical importance. Nutritional status and nutritional intervention are understudied subjects in PAH and an unmet medical need in clinical practice, since targeted nutritional intervention is currently not applied in PAH treatment, and there exists little knowledge about nutritional status of PAH patients. However, metabolic alterations may contribute to the poor quality of life in PAH patients (Vinke et al., 2018), and a wealth of evidence exists in other disorders to support that nutritional interventions can correct nutritional 
deficiencies and improve muscle function (Collins et al., 2012; van Norren et al., 2009; von Haehling et al., 2017). More specifically, up until now, the development of nutritional strategies that target both cardiac and skeletal muscle remodelling in PAH remains elusive.

To complicate matters further, inflammation is an important factor also contributing to pathological ventricular remodelling that underpins the development of fibrosis (Passino et al., 2015; Wynn \& Ramalingam, 2012). It has been consistently shown that the presence of chronic low-grade inflammation (CLGI) (e.g. shown from circulating TNF $\alpha$ and IL-6 levels) is linked to the development of cardiovascular disease (Calder et al., 2017) and to changes in body composition that negatively affect physical performance (Bowen et al., 2015; Calder et al., 2017; Klein et al., 2013). The use of fish oil-derived omega-3 poly-unsaturated fatty acids (PUFAs), in particular eicosapentaenoic acid (EPA) and docosahexaenoic acid (DHA) seems a promising option for the treatment of GLGI, because of their mild anti-inflammatory properties (Calder, 2017). Nutritional intervention with high protein, leucine, fish oil and oligosaccharides has been shown to have a beneficial effect on inflammation and skeletal muscle atrophy in a mouse model of cancer (van Norren et al., 2009). Given these effects, the present study aimed to determine if a nutritional intervention high in protein, fish oil and oligosaccharides could attenuate RV remodelling and skeletal muscle wasting in a PAH model. We induced PAH by injection of monocrotaline (MCT) for 8 weeks, which is a validated animal model for PAH in both male and female mice (Ahn et al., 2013; Bowen, Adams, et al., 2017). In the present study, female mice were selected in view of the sex-dependent incidence rate of $\mathrm{PAH}$ in humans.

\subsection{Materials \& Methods}

\subsubsection{Experimental diets}

The experimental diet was designed to test the effect of the combined nutritional intervention consisting of extra protein, leucine, oligosaccharides and fish oil added to the background diet AIN-93M (Research Diet Services, Wijk bij Duurstede, the Netherlands). The control diet was isocaloric to the experimental diet and contained per $\mathrm{kg}$ feed 110,3 g protein (casein), 53,6 g fat (corn oil), and 653,1 g carbohydrates. The isocaloric experimental diet (further referred to as Nutritional Intervention; NI) contained per $\mathrm{kg}$ feed: $210 \mathrm{~g}$ protein $(189 \mathrm{~g}$ intact protein of which $68 \%$ casein and 
$32 \%$ whey and $21 \mathrm{~g}$ free leucine), $57 \mathrm{~g}$ fat (20.2 $\mathrm{g}$ corn oil, $10.2 \mathrm{~g}$ rapeseed oil, and 22.2 $\mathrm{g}$ fish oil (providing $6.9 \mathrm{~g}$ EPA and $3.1 \mathrm{~g}$ DHA)), $561 \mathrm{~g}$ carbohydrates, $18 \mathrm{~g}$ galactooligosaccharides and $2 \mathrm{~g}$ fructo-oligosaccharides. Both diets were supplied as pellets.

\subsubsection{Animals and study design}

All experiments and procedures were approved by the local Animal Research Council, University of Leipzig and the Landesbehörde Sachsen (TVV 39/17). All experiments were performed in accordance with relevant guidelines and regulations.

This study included three groups of female C57BL/6 mice (aged 8 weeks) mice: 1) saline-treated (sham; n=9); 2) monocrotaline (MCT)-treated fed standard semisynthetic diet AIN-93M (MCT; 600 mg/kg; n=9); and 3) MCT-treated fed an isocaloric semi-synthetic diet with high protein, leucine, oligosaccharides and fish oil $(\mathrm{MCT}+\mathrm{NI} ; \mathrm{n}=10)$. MCT was given weekly by subcutaneous injection and sham mice received a matched volume of saline for 8 weeks. During that time period, MCT is known to induce pulmonary hypertension and RV dysfunction (Ahn et al., 2013; Bowen, Adams, et al., 2017). Mice were exposed to identical conditions under a 12:12 $\mathrm{h}$ light/dark cycle with food and water provided ad libitum. All groups received their treatment diet one week prior to the MCT injections. Body weight and food intake were recorded every week. Mice were sacrificed following deep anesthetization with i.p. administration of fentanyl $(0.05 \mathrm{mg} / \mathrm{kg})$, medetomidine $(0.5$ $\mathrm{mg} / \mathrm{kg})$, midazolam $(5 \mathrm{mg} / \mathrm{kg})$ and ketamine $(100 \mathrm{mg} / \mathrm{kg})$. At sacrifice, the heart, lungs, tibialis anterior (TA), soleus and extensor digitorum longus (EDL) were dissected, cleaned, blotted dry and weighed and tibia length was measured. A medial section of the heart was fixed in $4 \%$ PBS-buffered formalin. The RV was dissected from the left ventricle and immediately frozen in liquid $\mathrm{N}_{2}$ for RNA and protein analysis. The lungs were dried at $37^{\circ} \mathrm{C}$ to measure wet/dry lung weight.

\subsubsection{Tissue Analyses, RNA isolation and quantification of mRNA expression}

Total RNA was isolated from RV tissue and reverse transcribed into cDNA using random hexamers and Sensiscript Reverse Transcriptase (Qiagen, Hilden, Germany). An aliquot of the cDNA was used for quantitative RT-PCR. The expression of specific genes was normalized to the expression of hypoxanthin 
phosphoribosyltransferase (HPRT) mRNA. For quantification of expression of specific genes, primers listed in table 5.1 were used. For tumor necrosis factor alpha $(\mathrm{TNF} \alpha)$ commercial primers for SYBR Green assay were used (PrimePCR, Biorad laboratories).

Table 5.1: Sequences of quantitative RT-PCR primers

\begin{tabular}{|c|c|c|}
\hline Symbol & Forward primer sequence $\left(5^{\prime}-3^{\prime}\right)$ & Reverse primer sequence $\left(5^{\prime}-3^{\prime}\right)$ \\
\hline HPRT & $\begin{array}{l}\text { TCATGGACTGATTATGGAC } \\
\text { GGAC }\end{array}$ & $\begin{array}{l}\text { GCAGGTCAGCAAAGAACTT } \\
\text { ATAGCC }\end{array}$ \\
\hline ADAMTS-1 & $\begin{array}{l}\text { AAGTGAAGCCAGCCAGTAC } \\
\text { CA }\end{array}$ & TCCCGCAAGTTTTGGAACA \\
\hline VCAN & $\begin{array}{l}\text { ATCAATGGGAAGCAGCTCGT } \\
\mathrm{T}\end{array}$ & $\begin{array}{l}\text { GCATGGTAGTTGACGATTCT } \\
\text { GT }\end{array}$ \\
\hline TGFb1I1 & $\begin{array}{l}\text { GCCTCTGTGGCTCCTGCAAT } \\
\text { AAAC }\end{array}$ & $\begin{array}{l}\text { CTTCTCGAAGAAGCTGCTGC } \\
\text { СTC }\end{array}$ \\
\hline COL14a1 & $\begin{array}{l}\text { CAAAAATTCTGAGCCGCTAG } \\
\text { TT }\end{array}$ & $\begin{array}{l}\text { GAGTTTCCATTTGGTCCTCTT } \\
\text { G }\end{array}$ \\
\hline $\mathrm{COX}-2$ & $\begin{array}{l}\text { TGAGCAACTATTCCAAACCA } \\
\text { GC }\end{array}$ & $\begin{array}{l}\text { GCACGTAGTCTTCGATCACT } \\
\text { ATC }\end{array}$ \\
\hline
\end{tabular}

\subsubsection{Western blot analysis}

Frozen TA muscle samples were homogenized in relax buffer $(90 \mathrm{mmol} / \mathrm{L}$ HEPES, $126 \mathrm{mmol} / \mathrm{L} \mathrm{KCl}, 36 \mathrm{mmol} / \mathrm{L} \mathrm{NaCl}, 1 \mathrm{mmol} / \mathrm{L} \mathrm{MgCl}, 50 \mathrm{mmol} / \mathrm{L} \mathrm{EGTA}, 8 \mathrm{mmol} / \mathrm{L}$ $\mathrm{ATP}, 10 \mathrm{mmol} / \mathrm{L}$ creatine phosphate; $\mathrm{pH}$ 7.4) containing a protease inhibitor mix (inhibitor mix M, Serva, Heidelberg,Germany) and sonicated. Protein concentration was determined (bicinchoninic acid assay, Pierce, Bonn,Germany), and aliquots (5 $\mu \mathrm{g}$ ) were separated by SDS-polyacrylamide gel electrophoresis (10\% gels). Proteins were transferred to a polyvinylidene fluoride membrane and incubated overnight at $4^{\circ} \mathrm{C}$ with the following primary antibodies: MuRF1 (1/1000, Myomedix Ltd., Neckargemünd, Germany) or MuRF2 (1/1600, Myomedix Ltd., Neckargemünd, Germany). Membranes were subsequently incubated with a horseradish peroxidase-conjugated secondary antibody and specific bands visualized by enzymatic chemiluminescence (Super Signal West Pico, Thermo Fisher Scientific Inc.,Bonn, Germany) and densitometry quantified using a 1D scan software package 
(Vision-Capt, Vilber Lourmat, Eberhardzell, Germany). Blots were then normalized to the loading control GAPDH (1/30 000; HyTest Ltd., Turku, Finland).

\subsubsection{Histology}

Paraffin-embedded medial cross sections $(3 \mu \mathrm{m})$ of the heart, TA, soleus and EDL were mounted on glass slides. Subsequently, slides of the heart were stained with H\&E and Picrosirius red to assess right ventricle wall thickness and fibrosis by imaging software (ImageJ, 1.51d, NIH and Sigma Scan Pro 5.0, Systat Software Inc). Slides of the TA and EDL were stained with H\&E to assess muscle fiber crosssectional area by imaging software (ImageJ, 1.51d, NIH). Fiber type and crosssectional area (CSA) of the soleus were analyzed by immunohistochemistry; after target retrieval with Signal Stain Citrate solution (Cell Signaling), the slides were covered with proteinase K solution (Sigma, P2308), peroxidase and protein block (Dako), then incubated with anti-slow skeletal myosin heavy chain (Abcam, ab11083) in antibody dilution with background reducing factor (Dako) and antimouse HRP (Sigma 9044) in antibody dilution with background reducing factor (Dako). Development was performed with DAB+ coloring agent (Dako) and nuclei were colored with Mayer's Hematoxylin Solution.

\subsubsection{Microarray analysis}

Total RNA from the right ventricle was isolated using trizol. RNA concentrations were measured by absorbance at $260 \mathrm{~nm}$ (nanodrop). RNA quality was checked using the RNA 6000 nano kit assay on the Agilent 2100 bioanalyser (Agilent Technologies, Amsterdam, The Netherlands) according to the manufacturer's protocol. For each mouse, total RNA (100 ng) was labelled using the Affymetrix WT plus reagent kit (Life Technologies, Bleiswijk, The Netherlands).

Microarray experiments were performed using Affymetrix mouse gene 1.1 ST arrays. From the experiment, 3 control samples, 3 MCT samples and $3 \mathrm{MCT}+\mathrm{NI}$ samples that were matched for disease severity were included in this experiment. Array data were analyzed using an in-house, online system (Lin et al., 2011). Arrays passed quality control, so no samples had to be excluded from analysis. Briefly, probe sets were redefined according to Dai et al. (Dai et al., 2005), using remapped computable document format version 19 based on the Entrez gene database. In total these arrays target 21.114 unique genes. Normalized expression estimates were 
obtained from the raw intensity values using the robust multi-array analysis (RMA) preprocessing algorithm available in the library 'affyPLM' using default settings (Irizarry et al., 2003). Differentially expressed probe sets were identified using linear models, applying moderated $t$-statistics that implemented intensity-based empirical Bayes regularization of standard errors (library 'limma'). The moderated t-test statistic has the same interpretation as an ordinary t-test statistic, except that the standard errors have been moderated across genes, i.e. shrunk to a common value, using a Bayesian model (Ritchie et al., 2015; Sartor et al., 2006). Probe sets that satisfied the criterion of $\mathrm{P}<0.01$ or $\mathrm{P}<0.05$ (depending on the research question) were considered to be significantly regulated.

Changes in gene expression were related to biologically meaningful changes using gene set enrichment analysis (GSEA) of broadinstitute.org (Subramanian et al., 2005). Gene sets were retrieved from the expert-curated Kyoto Encyclopedia of Genes and Genomes (KEGG), Biocarta, Reactome and WikiPathways pathway databases. Only gene sets consisting of more than 15 and fewer than 500genes were taken into account, which resulted in the inclusion of 1,382 gene sets. For each comparison, genes were ranked on their t-value that was calculated by the empirical Bayes method. Statistical significance of GSEA results was determined using 1,000 permutations.

The array data has been submitted to the Gene Expression Omnibus (GEO) (Edgar et al., 2002), under accession number GSE125537.

\subsubsection{Statistical analyses}

Data are presented as mean \pm SD. One-way analysis of variance (ANOVA) followed by Tukey post hoc was used to compare groups (GraphPad Prism). Significance was accepted as $\mathrm{P}<0.05$. 


\subsection{Results}

\subsubsection{Weight development}

Consistent with previous data (Bowen, Adams, et al., 2017), MCT injections over an 8 week period resulted in a significantly reduced body weight increase over time when compared to sham animals. The increase in body weight was $7.4 \pm 1.5 \%$ for sham mice, whereas MCT mice only increased weight by $2.3 \pm 3.2 \%$ and MCT mice receiving nutritional intervention $(\mathrm{MCT}+\mathrm{NI})$ increased weight by $2.1 \pm 4.9 \%$ : (figure 5.1A). The change in body weight was not due to a lower food intake in the MCT group compared to the sham group, as cumulative food intake of the mice was identical at the end of the intervention (see appendix III).

\subsubsection{Development of pulmonary congestion and cardiac remodeling}

Compared to sham mice, MCT mice showed an increase in pulmonary congestion (figure 5.1B), heart weight corrected for tibia length $(6.30 \pm 0.62 \mathrm{mg} / \mathrm{mm}$ vs $5.95 \pm 0.29$ $\mathrm{mg} / \mathrm{mm}$ ) (figure $5.1 \mathrm{C}$ ) and right ventricular thickness $(0.53 \pm 0.03 \mathrm{~mm}$ vs $0.48 \pm 0.05$ $\mathrm{mm}$ ) (figure 5.1D) (all $\mathrm{p}<0.05)$. These are signs of the development of pulmonary hypertension and subsequent RV hypertrophy in the MCT group. In the MCT+NI group, heart weight was not different from sham mice and reduced compared to MCT mice $(p<0.01)$ (figure 5.1C). Pulmonary congestion was increased in the $\mathrm{MCT}+\mathrm{NI}$ group compared to sham mice $(\mathrm{p}<0.05)$ and not different from the MCT group (figure 5.1B). RV thickness in the MCT+NI group was attenuated compared to the MCT group, such that no difference was detected compared to sham mice (figure 5.1D). There were no significant changes in the thickness of the left ventricle and the septum. 
A

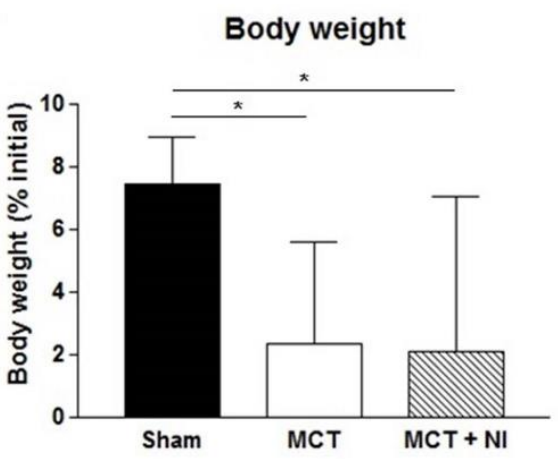

C

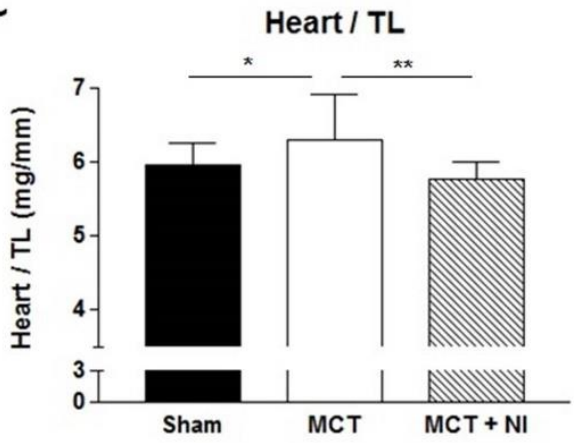

B

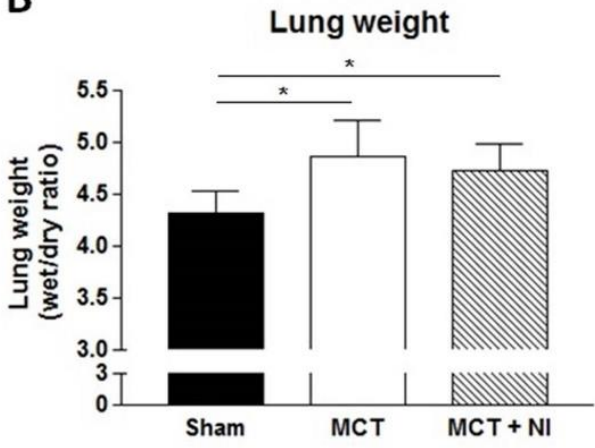

Right ventricle thickness

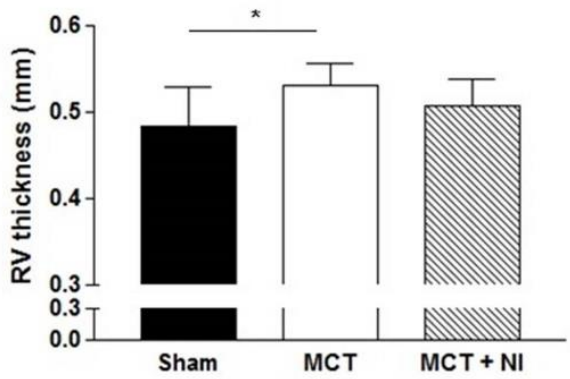

Figure 5.1: Total body weight development, lung weight and cardiac hypertrophy after 8 weeks of MCT injection. A) Compared to sham mice, relative body weight was impaired $(p<0.05)$ in MCT and MCT+NI by $5 \%$. B) Both MCT groups showed an increase in lung weight ( $<<0.05)$ compared to shams. $C)$ An increase in heart weight of MCT mice compared to shams was also seen, however these alterations were attenuated in MCT+NI mice. D) Right ventricle thickness was increased only in MCT mice. Scatterplots showing variation of the data represented in these graphs are depicted in appendix IV.

$\left({ }^{*} \mathrm{p}<0.05,{ }^{* *} \mathrm{p}<0.01\right)$

\subsubsection{Anti-fibrotic effect of the nutritional intervention on the right ventricle}

Histological analysis showed a 1.6 fold change increase in fibrosis of the RV in the MCT group compared to sham $(\mathrm{p}<0.05)$, that was normalized in the group receiving nutritional intervention $(\mathrm{p}<0.05)$ (fold change of 1.02 versus sham) (figure 5.2A-B). Comparison of genes from the microarray analysis showed 245 genes that were 
differentially regulated $(\mathrm{p}<0.05)$ when comparing MCT vs Sham and MCT+NI vs MCT group (figure 5.3A), with several of these genes involved in fibrosis. Using a cut-off of $\mathrm{p}<0.01,32$ significantly differentially expressed genes were detected (figure 5.3B). Two of these genes are involved in fibrotic pathways (TGFb1I1 and ADAMTS-1).
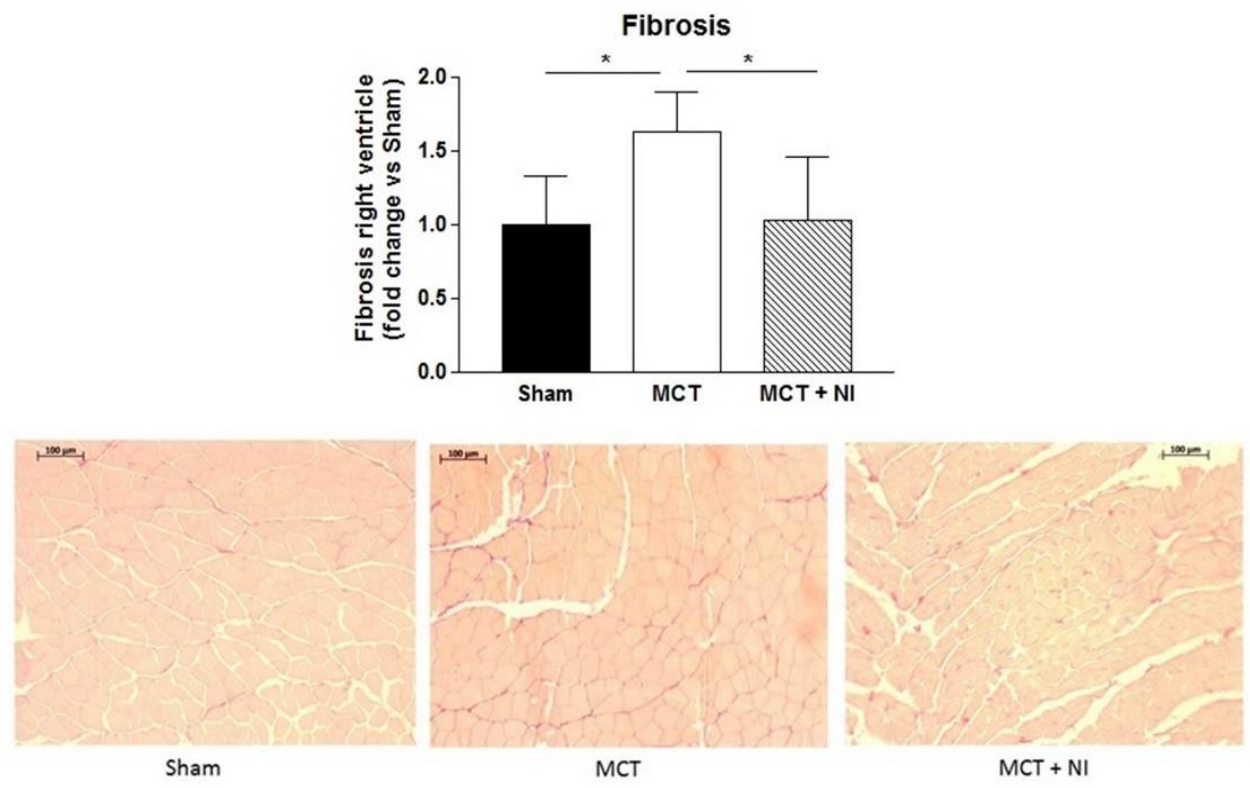

Figure 5.2: Nutritional intervention prevents increased right ventricular fibrosis after MCT injection. Right ventricular fibrosis was increased in MCT mice $(p<0.05)$ compared to shams and attenuated in MCT + NI mice. A scatterplot showing variation of the data represented in this graph is depicted in appendix IV $(* \mathrm{p}<0.05)$

Gene Set Enrichment Analysis (GSEA) of the RV of a representative subset of three mice showed overrepresentation of fibrotic pathways upon MCT injection (MCT vs. Sham) (no FDR q-value cut-off was used; the complete datasets were added as supplementary data, see appendix V). Upon nutritional intervention, fibrotic pathways were underrepresented in MCT+NI versus MCT (supplementary data, see appendix V). GSEA of the RV also showed underrepresentation of pathways involved in energy metabolism and mitochondrial function in MCT versus Sham. 
Upon nutritional intervention, these pathways were overrepresented (supplementary data, see appendix V).

A

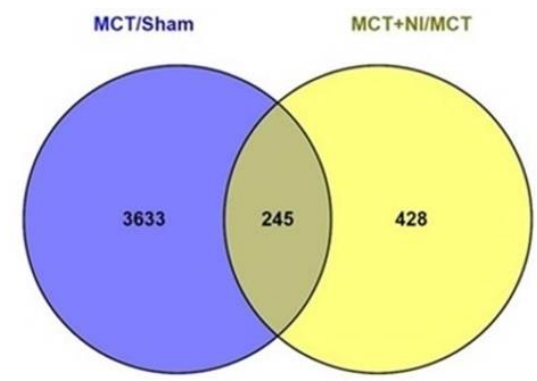

B

\begin{tabular}{|c|c|c|c|c|c|c|c|c|c|c|c|}
\hline Entrez & Gene & sham & & & MCT & & & $\mathrm{MCT}+\mathrm{NI}$ & & & Description \\
\hline 16628 & Kira10 & 0.219 & .0 .135 & .0 .083 & & 0.945 & 0.587 & -0.221 & -0.053 & -0.072 & killer cell lectin-like receptor subfamily $A$, member 10 \\
\hline 258139 & Olft591 & 0.005 & 0.091 & -0.096 & 1.127 & 0.791 & 0.298 & 0.078 & -0.169 & 0.193 & olfactory receptor 591 \\
\hline 20672 & Sox18 & 0.031 & 0.012 & .0 .043 & 0.685 & 0.651 & 0.734 & 0.920 & 0.949 & 0.882 & SRY (sex determining region Y)-box 18 \\
\hline 21804 & Tgfb1i1 & -0.022 & -0.087 & 0.109 & 0.488 & 0.706 & 0.623 & 0.338 & 0.299 & 0.406 & transforming growth factor beta 1 induced transcript 1 \\
\hline 237877 & Atad5 & -0.019 & 0.090 & -0.071 & 0.488 & 0.617 & 0.538 & 0.313 & 0.318 & 0.313 & ATPase family, AAA doma in containing 5 \\
\hline 11504 & Adamts1 & -0.017 & 0.103 & .0 .086 & 0.432 & 0.434 & 0.621 & 0.241 & 0.228 & 0.227 & ADAMTS-1 \\
\hline 233893 & Zfp764 & .0 .002 & -0.160 & 0.163 & 0.377 & 0.673 & 0.407 & 0.163 & -0.081 & -0.218 & zinc finger protein 764 \\
\hline 108030 & Lin7a & 0.144 & -0.144 & 0.001 & 0.515 & 0.432 & 0.428 & 0.707 & 0.868 & 0.697 & lin-7 homolog A (C. elegans) \\
\hline 236749 & Gm4907 & -0.021 & 0.016 & 0.006 & 0.603 & 0.537 & 0.199 & -0.027 & 0.160 & -0.153 & predicted gene 4907 \\
\hline 20893 & Bhihe40 & .0 .088 & .0 .055 & 0.143 & 0.321 & 0.593 & 0.295 & 0.021 & 0.136 & -0.013 & basic helix-loop-helix family, member e 40 \\
\hline 192185 & Nadk & 0.046 & -0.067 & 0.021 & 0.366 & 0.346 & 0.389 & 0.224 & 0.251 & 0.239 & NAD kinase \\
\hline 20600 & $5 \mathrm{mr} 2$ & -0.077 & 0.042 & 0.035 & 0.277 & 0.446 & 0.253 & -0.097 & 0.092 & 0.042 & submaxillary gland androgen regulated protein 2 \\
\hline 216144 & Vmn2r81 & -0.004 & 0.027 & -0.023 & 0.245 & 0.481 & 0.236 & 0.065 & -0.079 & 0.091 & vomeronasal 2 , receptor 81 \\
\hline 72054 & Cyp4f18 & 0.032 & -0.122 & 0.090 & 0.312 & 0.312 & 0.219 & .0 .010 & 0.015 & 0.090 & cytochrome P450, family 4, subfamily f, polypeptide 18 \\
\hline 13629 & Eef2 & 0.014 & 0.003 & -0.017 & 0.061 & 0.112 & 0.106 & 0.024 & -0.013 & 0.020 & eukaryotic translation elongation factor 2 \\
\hline 21924 & Tnnc1 & -0.028 & 0.030 & .0 .003 & 0.099 & 0.099 & 0.074 & 0.206 & 0.183 & 0.160 & troponin C, cardiac/slow skeletal \\
\hline 23980 & Pebp1 & -0.006 & 0.024 & -0.017 & -0.137 & -0.067 & -0.164 & 0.082 & 0.065 & 0.049 & phosphatidylethanolamine binding protein 1 \\
\hline 227095 & Hibch & .0 .006 & 0.030 & -0.024 & -0.118 & -0.192 & -0.121 & -0.015 & 0.098 & 0.059 & 3-hydroxyisobutyryl-coenzyme A hydrolase \\
\hline 235169 & Foxred 1 & -0.023 & .0 .011 & 0.035 & -0.230 & -0.089 & .0 .155 & 0.014 & 0.039 & -0.023 & FAD-dependent oxidoreductase domain containing 1 \\
\hline 66191 & |er3ip1 & -0.020 & -0.011 & 0.031 & -0.245 & -0.170 & -0.070 & 0.026 & 0.052 & 0.054 & immediate early response 3 interacting protein 1 \\
\hline 72278 & Cepg1 & .0 .002 & 0.004 & .0 .002 & .0 .179 & -0.215 & -0.218 & -0.080 & -0.110 & .0 .134 & cell cycle progression 1 \\
\hline 21672 & $\operatorname{Prdx2}$ & 0.049 & -0.046 & -0.003 & -0.175 & .0 .260 & -0.186 & -0.049 & 0.153 & -0.060 & peroxiredoxin 2 \\
\hline 68198 & Ndufb2 & .0 .002 & 0.034 & .0 .033 & .0 .344 & .0 .164 & -0.160 & 0.024 & -0.018 & 0.083 & NADH dehydrogenase (ubiquinone) 1 beta subcomplex, 2 \\
\hline 215708 & Fam73a & 0.017 & 0.046 & -0.062 & .0 .267 & -0.235 & -0.184 & -0.407 & -0.454 & -0.355 & family with sequence similarity 73 , member $A$ \\
\hline 246221 & Mpst & 0.005 & 0.065 & .0 .070 & -0.277 & -0.214 & .0 .312 & -0.052 & 0.046 & -0.075 & mercaptopyruvate sulfurtransferase \\
\hline 15408 & Hoxb13 & 0.031 & -0.069 & 0.038 & .0 .259 & -0.293 & -0.356 & 0.092 & 0.038 & .0 .069 & homeobox 813 \\
\hline 268319 & BC025920 & -0.052 & 0.104 & -0.052 & -0.414 & -0.353 & -0.142 & -0.052 & 0.050 & 0.045 & zinc finger protein pseudogene \\
\hline 102638837 & LOC102638837 & .0 .063 & 0.034 & 0.029 & .0 .332 & -0.202 & -0.452 & .0 .063 & -0.024 & -0.033 & transducin-like enhancer protein 2-like \\
\hline 258869 & Olfr147 & 0.086 & 0.067 & -0.153 & -0.430 & -0.378 & -0318 & 0.012 & -0.153 & 0.083 & olfactory receptor 147 \\
\hline 628737 & Gm6909 & 0.077 & .0 .018 & -0.059 & .0 .407 & -0.592 & .0 .453 & -0.082 & -0.310 & -0.165 & predicted gene 6909 \\
\hline 664857 & Cfap46 & -0.200 & -0.029 & 0.229 & .0 .655 & -0.559 & -0.336 & 0.077 & 0.027 & -0.126 & cilia and flagella protein 46 \\
\hline 17171 & Mas1 & -0.051 & 0.074 & .0 .023 & .0 .678 & -0.466 & -0.745 & -0.393 & .0 .284 & -0.305 & MAS1 oncogene \\
\hline
\end{tabular}

Figure 5.3: Differential expression of genes in the right ventricle after MCT injection. A) Heatmap showing 32 genes that are differentially expressed in MCT compared to sham and in MCT+NI compared to MCT, using a cutoff of $p<0.01$. B) A total of 245 genes were differentially expressed in MCT compared to Sham and MCT+NI compared to MCT, using a cutoff of $\mathrm{p}<0.05$.

\subsubsection{Validation of the microarray data on right ventricular fibrosis}

Next, we validated the two fibrotic genes (TGFb1I1 and ADAMTS-1) with a significance level of $p<0.01$ in the microarray analysis in all our samples by qRTPCR. We also validated two genes with a significance level of $\mathrm{p}<0.05$ (VCAN and Col14a1) by qRT-PCR. This confirmed upregulation of fibrotic genes in the right 
ventricle in MCT vs Sham and downregulation in MCT+NI vs MCT (figure 5.4). MCT injection induced a 2-fold increase in VCAN mRNA expression relative to sham mice $(\mathrm{p}<0.001$, figure $5.4 \mathrm{~A})$. In MCT-injected mice receiving nutritional intervention, this increase was $80 \%$ lower ( $p<0.01$, figure 5.4A). For ADAMTS- 1 , the effect was similar, with a 2.5 -fold increase in MCT versus sham mice $(p<0.001)$ and only 1.25 -fold increase in MCT-injected mice receiving nutritional intervention ( $<<0.05$, figure 5.4B). Col14a1 mRNA expression was increased 1.35-fold in MCTinjected mice relative to sham ( $\mathrm{p}<0.05)$, although the expression normalized in MCT mice receiving nutritional intervention ( $p<0.05$, figure $5.4 \mathrm{C})$. mRNA expression of TGFb1I1 was increased by $65 \%$ in MCT-injected mice compared to sham $(\mathrm{p}<0.05)$. The increase in MCT-injected mice receiving nutritional intervention showed no significant effect $(p=0.18$, figure $5.4 \mathrm{D})$.

A

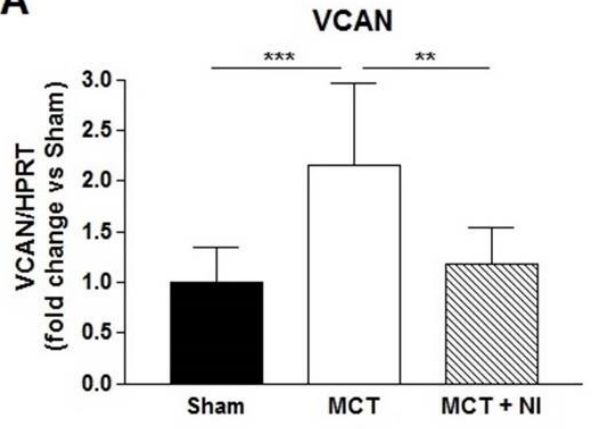

C

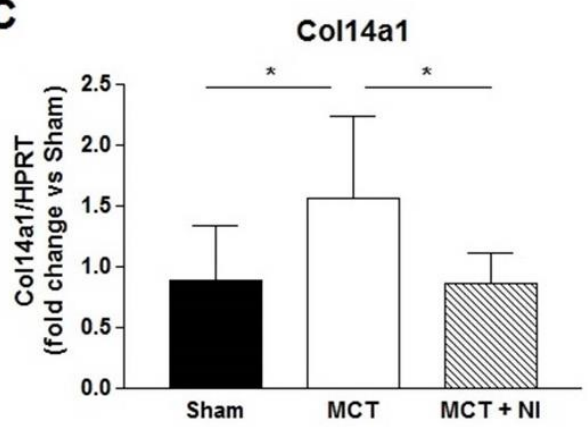

B

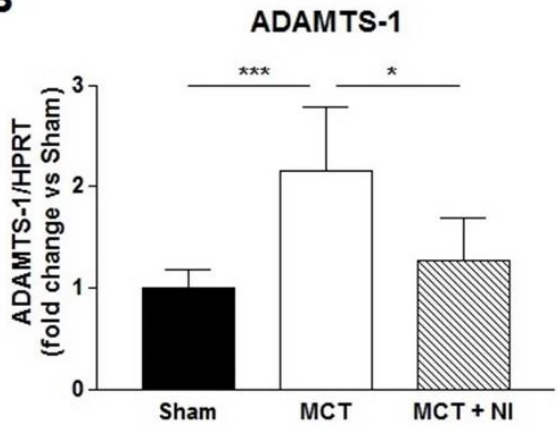

D

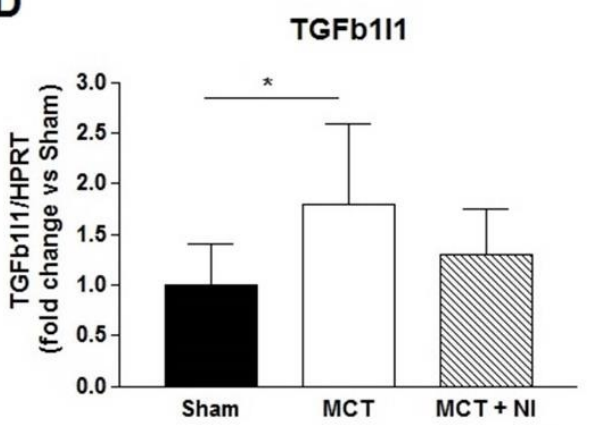

Figure 5.4: Fibrotic genes were upregulated in MCT, but not in MCT+NI goup. Compared to sham mice, mRNA levels of genes involved in fibrosis were upregulated in MCT mice, but not in MCT + NI mice $\left({ }^{*} \mathrm{p}<0.05,{ }^{* *} \mathrm{p}<0.01,{ }^{* * *} \mathrm{p}<\right.$ 0.001). (VCAN: versican; ADAMTS-1: a disintegrin and metalloproteinase with 
thrombospondin motifs 1; Col14a1: collagen type XIV alpha 1; TGFb1I1: transforming growth factor beta 1 induced transcript 1 )

5.3.5 Effect of nutritional intervention on inflammation markers in the right ventricle

Next to higher mRNA levels of fibrotic genes, we could also show that MCT injection induced a 5.5-fold increased level of tumor necrosis factor alpha (TNF $\alpha)$ in the right ventricle of MCT mice relative to sham mice ( $\mathrm{p}<0.05$, figure 5.5A). In MCT-injected mice receiving the nutritional intervention, this increase was only 2.26 fold (figure 5.5A). COX-2 mRNA levels also tended to be increased in right ventricle of MCT compared to sham and $\mathrm{MCT}+\mathrm{NI}$ mice, although the effect was not significant $(p=0.068$, figure $5.5 B)$. These results suggest an anti-inflammatory effect of the nutritional supplement.

A

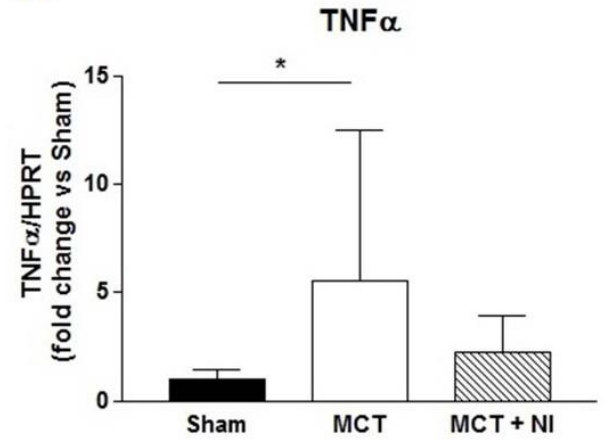

B

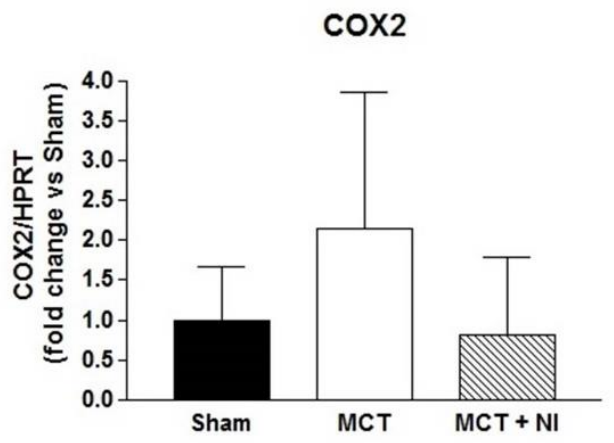

Figure 5.5: Nutritional intervention reduces an increase in TNF $\alpha$ mRNA levels in the right ventricle. A) MCT injection induced a 5.5-fold increased level of TNF $\alpha$ mRNA in the right ventricle of MCT relative to sham mice. In MCT+NI mice this increase was only 2.26 fold. B) COX-2 mRNA levels tended to be increased in right ventricle of MCT compared to sham mice ( $p$ 0.068)

TNF $\alpha$ : tumor necrosis factor alpha; COX2: cyclooxygenase-2. $\left({ }^{*} \mathrm{p}<0.05\right)$

\subsubsection{Effect of nutritional intervention on the skeletal muscle}

The weight of the tibialis anterior (TA) muscle was similar in all groups (figure 5.6A), however fiber cross-sectional area (CSA) of the TA muscle was reduced $(\mathrm{P}<0.05)$ by $22 \%$ in MCT compared to sham mice, but preserved in the MCT+NI group (1503 vs. 
1178 vs $1495 \mu \mathrm{m}^{2}$, respectively) (figure 5.6B). Protein expression of the key E3 ligase MuRF1 was reduced by $30 \%$ in the group receiving nutritional intervention compared to MCT mice alone $(\mathrm{p}<0.05)$ (figure 5.7A). The E3 ligase MuRF2 was increased by $41 \%(\mathrm{p}<0.05)$ in the MCT group compared to sham. Nutritional intervention reduced MuRF2 protein levels by $28 \%(\mathrm{p}<0.05)$ (figure $5.7 \mathrm{~B})$. The weight of the extensor digitorum longus (EDL) muscle was similar in all groups (figure 5.6C). However, fiber cross-sectional area was reduced by $29 \%(\mathrm{p}<0.05)$ in the MCT mice compared to sham, and preserved in the MCT+NI group (764 vs 542 vs 742 $\mu \mathrm{m}^{2}$, respectively) (figure 5.6D). The weight of the soleus muscle was also similar in all groups (figure 5.6E), with no effect of MCT or nutritional treatment on muscle fiber cross-sectional area (figure 5.6F).

A

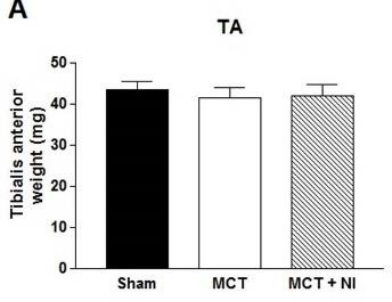

B

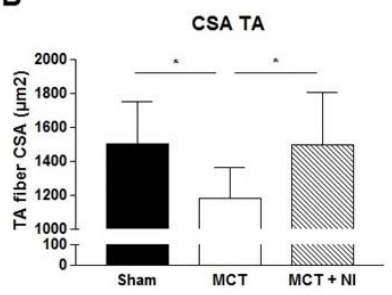

C

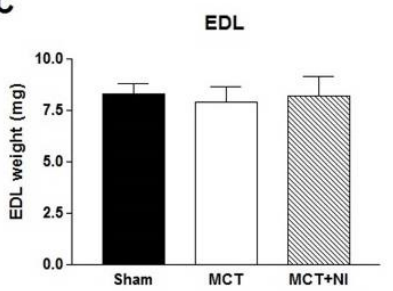

D

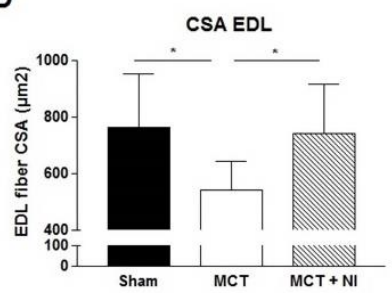

E

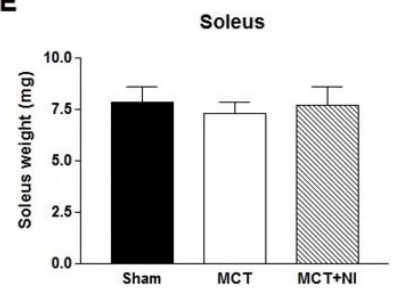

$\mathbf{F}$

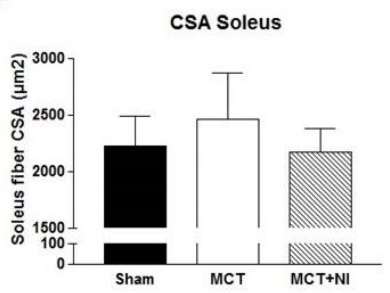

Figure 5.6: Changes in skeletal muscle weight and muscle fiber cross-sectional area. A) Weight of the tibialis anterior (TA) muscle was unchanged, B) but muscle fiber cross-sectional area (CSA) of the TA was reduced by $22 \%$ in MCT mice compared to sham, but unaltered in MCT+NI. B) Weight of the EDL muscle was unchanged, but D) skeletal muscle fiber CSA of the EDL was reduced by $29 \%$ in the MCT mice compared to sham and preserved in the MCT+NI group $\left({ }^{*} p<0.05\right)$. E) Weight of the soleus muscle was similar in all groups and F) skeletal muscle fiber CSA was also unchanged 
A
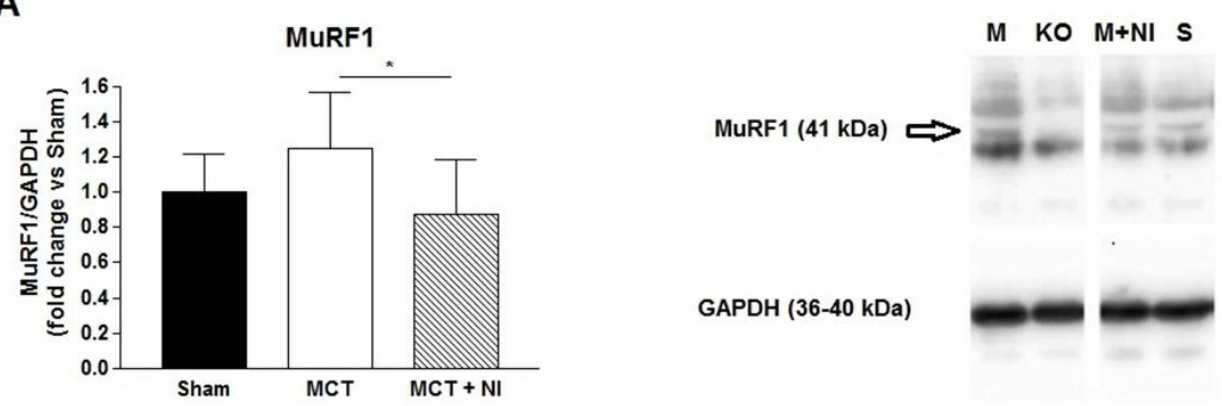

B
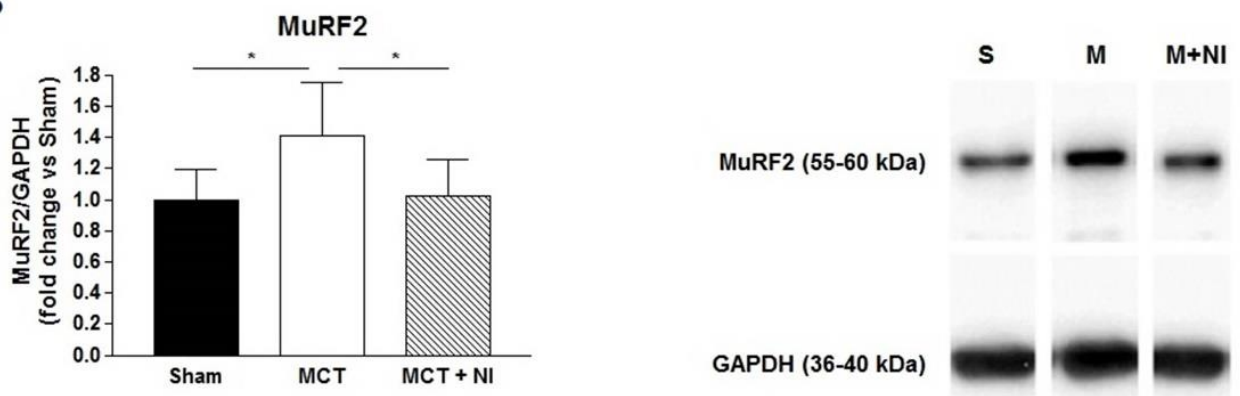

Figure 5.7: An increase in E3 ubiquitin-protein ligases MuRF1 and MuRF2 in MCT mice is prevented by nutritional intervention. A) Protein levels of the key E3 ligase MuRF1 was reduced by $30 \%$ in the group receiving nutritional intervention compared to MCT mice alone. B) The E3 ligase MuRF2 was increased by $41 \%$ in the MCT group compared to sham. Nutritional intervention reduced MuRF2 protein levels by $28 \%$. $\left.{ }^{*} \mathrm{p}<0.05\right)$

Pictures are cropped from full-length pictures of blots. Full-length blots are presented in appendix VI. A MuRF1 knock-out (KO) sample was included to separate the MuRF1 bands from other bands due to nonspecific binding of the antibody.

\subsection{Discussion}

The objective of this study was to assess the impact of a targeted nutritional intervention on both cardiac and skeletal muscle remodeling in a PAH model induced by MCT in mice. Indeed, adding more protein, leucine, fish oil and oligosaccharides to the feed caused an attenuation of RV hypertrophy, fibrosis and inflammation, while simultaneously preventing skeletal muscle atrophy. To our 
knowledge, this is the first study to show that PAH-associated pathological changes in both cardiac and skeletal muscle can be attenuated simultaneously by multipletarget nutritional intervention.

This is of great clinical importance, since current treatment options do not target both the cardiac and skeletal muscle alterations in PAH. If nutritional intervention can prevent such pathological changes and reduce the development of exercise intolerance leading to an impaired quality of life, this is a important step forward. Further studied are needed to check whether targeted nutritional intervention can improve physical status and wellbeing in PAH patients.

Eight weekly injections with MCT induced a clear PAH phenotype in mice with RV hypertrophy. Histological analysis of the RV showed an increase in fibrotic tissue in the mice receiving MCT-injections which clearly indicates cardiac remodeling typical of PAH (de Jesus Perez, 2016; Voelkel et al., 2014). The MCT mice receiving nutritional intervention did not show increased RV hypertrophy nor fibrosis. Microarray analysis of the RV and subsequent verification of the results by qRT-PCR showed that nutritional intervention prevented an increase of mRNA levels of fibrotic genes after MCT-injection in nearly all four genes. Probably the study was not powered to detect significant changes in all the genes studied. Microarray GSEA also showed downregulation of pathways involved in energy metabolism and mitochondrial function in mice receiving MCT-injections. Metabolic remodeling is another symptom of PAH. A switch from fatty acid metabolism and oxidative phosphorylation to the energetically inefficient aerobic glycolysis (also called the Warburg effect), is often seen (Archer et al., 2013; Samson \& Paulin, 2017; Voelkel et al., 2014). Our GSEA clearly showed the downregulation of genes in energy metabolism and mitochondrial function in MCT compared to sham mice, suggesting a metabolic switch from FA oxidation to glycolysis. It also showed that this effect was reduced in the MCT group receiving the nutritional intervention. A possible explanation for these findings is a reduction in inflammation due to the effect of added omega 3 fatty acids EPA and DHA. The mRNA levels of TNF $\alpha$ in the RV of the MCT mice were higher compared to sham mice. This effect was reduced upon nutritional intervention. This theory is supported by other studies using the monocrotaline model in male rats, showing reduction of PAH symptoms when antiinflammatory treatments were provided. Anti-inflammatory drugs such as acetylsalicylic acid (aspirin) (Gao et al., 2017; Shen et al., 2011) or 5-aminosalicylic 
acid (Sun et al., 2017) have produced improvements in right ventricular systolic pressure (RVSP), RV hypertrophy and pulmonary artery remodeling. Next to this, there are also indications that certain nutritional supplements with antiinflammatory properties including resveratrol (Csiszar et al., 2009; Koyama et al., 2008; Paffett et al., 2012; Zhou et al., 2015) and betaine (Yang et al., 2018) reduce the effects of PAH in the MCT rat model. Supplementation with branched chain aminoacids has been described to preserve cardiac function and prolonged survival in a cancer cachexia mouse model (Tanada et al., 2015). Moreover, a diet high in leucine provided after a myocardial infarction in mice was reported to decrease fibrosis and apoptosis, and improve cardiac structure (Witham et al., 2013).

Injection with MCT induced a reduction of muscle fiber CSA in the TA and EDL, but did not lead to alterations in the soleus. This might be due to the fact that the soleus has a more oxidative phenotype, reflected by a higher content of mitochondria, which might provide a certain level of protection against muscle wasting (Romanello \& Sandri, 2016). In addition, the soleus is more resistant to fatigue due to a relatively lower presence of glycolytic type II (fast twitch) muscle fibers, which are predominant in the EDL and TA (Augusto et al., 2004). The nutritional intervention was able to prevent the reduction in muscle fiber CSA in TA and EDL, indicating an anti-atrophic effect. This was confirmed by a reduced upregulation of the E3 ligases MuRF1 and MuRF2, which promotes muscle wasting by increasing protein degradation via ubiquitination (Koyama et al., 2008).

Faber et al reported that the same nutritional combination as used in our study reduced plasma TNF $\alpha$ and prostaglandin $\mathrm{E} 2\left(\mathrm{PGE}_{2}\right)$ in a mouse cancer cachexia model (Faber et al., 2008). Both PGE2 and TNF $\alpha$ have been reported to induce MURF1 induction, leading to elevated proteolysis and increased protein breakdown 12, 37. When TNF $\alpha$ is decreased due to attenuated inflammatory tone, protein breakdown in the skeletal muscle is decreased, explaining the effects in the TA. Furthermore, skeletal muscle protein synthesis is stimulated by branched chain amino acids, especially leucine (Kobayashi et al., 2006), which was added to the nutritional intervention. The effect on the skeletal muscle can be direct, by a reduction of inflammation and therefore protein breakdown in the muscle itself, or indirect by a reduction in disease severity. 
Chronic low-grade inflammation (CLGI) is an important part of the pathophysiology of PAH (Rabinovitch et al., 2014). Potential triggers for CLGI in PAH include chronic hypoxia, mechanical stretch, mitochondrial dysfunction and systemic complications such as changes in intestinal functioning (barrier function, dysbiosis etc.) (Calder et al., 2011; Klein et al., 2013; Pullamsetti et al., 2011). Chronic inflammation and expression of pro-inflammatory cytokines such as interleukin 6 (IL-6) and TNF $\alpha$ are known to contribute to muscle wasting which is also seen in PAH (Cohen et al., 2015; Manders et al., 2015; von Haehling et al., 2017). Fish oil containing (EPA) and (DHA) can influence inflammatory processes by changing the production of pro- or anti-inflammatory cytokines (Calder, 2015). Other nutritional components, such as galacto- and fructo-oligosaccharides, can restore the microbial balance in case of dysbiosis (Calder et al., 2017; Fernandes et al., 2017) and also have anti-inflammatory and immune-regulating effects (Macfarlane et al., 2008). Galactoand fructo-oligosacharides have been shown to be able to restore $\mathrm{T}$ cell function during chronic inflammation in a murine tumor model when combined with omega3 fatty acids and high quality and quantity protein (Faber et al., 2008). Increased intake of high quality protein and the anabolic amino acid leucine can also help prevent muscle wasting (Kobayashi et al., 2006; van Norren et al., 2009), which is why the combination of extra protein, leucine, fish oil and oligosaccharides was chosen for this study. It is unclear whether it is the whole combination or just a subset of the nutrients from the nutritional intervention that is responsible for the effects observed in this study. Further studies are warranted to reveal this aspect.

The model used for this study is an adapted version of our recently developed cachectic mouse model with monocrotaline-induced RV heart failure (Bowen, Adams, et al., 2017). The adaptation concerns the control diet and the length of time the animals are in the experiment. The result is a model with similar RV remodeling but a more modest loss of body weight, which more closely resembles the clinical situation in PAH. The presence of both pulmonary congestion and RV hypertrophy, shows that this method produces effects comparable to those seen in the male rat model (Gomez-Arroyo et al., 2012) that is widely accepted as a model for PAH. RV thickness increased, but we found no change on the septum and thickness of the left ventricle, showing that the monocrotaline mostly affects the RV. An additional advantage of the present model is that it concerns female animals, which is more in 
line with the notion that PAH occurs mostly in women (de Jesus Perez, 2016; Galiè, Humbert, et al., 2015).

\subsection{Conclusion}

A multi-compound nutritional intervention providing higher amounts of protein, leucine, fish oil and oligosaccharides significantly attenuated cardiac and skeletal muscle changes in a female mouse model of pulmonary hypertension. The effect on the development of fibrosis in the RV was the most profound and is at least partly due to anti-inflammatory effects mediated via TNF $\alpha$ and COX2. These results provide directions for further study to develop novel therapeutic strategies to prevent pathophysiological alterations in pulmonary hypertension.

\section{Acknowledgements}

We would like to thank Angela Kricke for helping with the histological analysis on the EDL and soleus and Guido Hooiveld for helping with the micro-array analysis. Paulien Vinke is grateful to have received research grants from the German Academic Exchange Service (DAAD) in Germany and the Ministry of Education, Culture and Science (OCW) in the Netherlands and the Fonds dr. Catharine van Tussenbroek in the Netherlands to perform this research. The funding source had no role in the study design, collection, analysis and interpretation of data, the writing of the report or in the decision to submit the article for publication. 


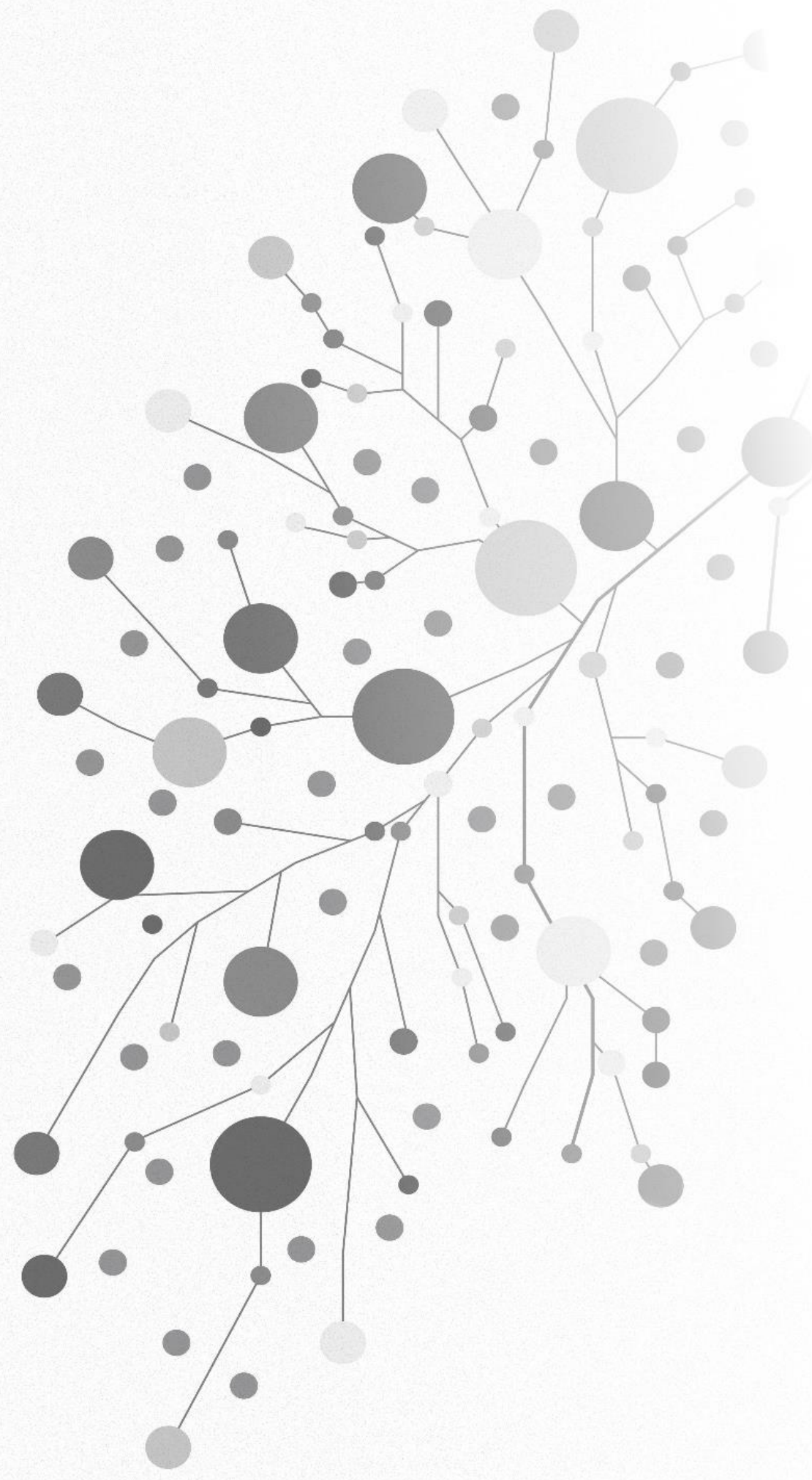


6 Synthesis, discussion and conclusion 


\subsection{Objectives and structure of the thesis}

In this thesis I focused on the knowledge gaps that exist regarding the possible connections between PAH pathophysiology, drug medication use, nutritional status and micronutrient deficiencies, muscle mass and function and exercise tolerance from different perspectives.

The main objectives of this thesis were to gain more insight in the prevalence of micronutrient deficiencies of patients with pulmonary hypertension and to obtain more knowledge on the potential usefulness of nutritional interventions aiming to reduce fatigue and improve exercise tolerance and quality of life in PAH patients. The research questions were:

1) What is currently known about the nutritional status of patients with pulmonary hypertension and how does the pathophysiology of $\mathrm{PAH}$ impact the relation between nutritional status, exercise intolerance and fatigue?

2) What is the prevalence of micronutrient deficiencies related to exercise tolerance and fatigue in patients with pulmonary hypertension and can deficiencies in certain micronutrients be related to clinical and patientrelated outcomes?

3) Can the use of certain medications, such as proton pump inhibitors, contribute to a reduction in muscle mass and function in chronically ill patients?

4) Can nutritional intervention reduce cardiac and skeletal muscle changes in PAH?

In this final chapter, findings as shown in the previous chapters will be summarized and synthesized. Furthermore, I will reflect on how the answers regarding the above-mentioned research questions relate to implementation of possible nutritional interventions in PAH treatment and lastly, I will discuss possible future research directions. This chapter consists of 5 sections $(6.2-6.6)$ centred around propositions on nutritional therapy for PAH patients. Section 6.7 contains the conclusions and a reflection on the overall objectives of this thesis. 


\subsection{Nutritional therapy is a promising emerging field in the treatment of PAH}

Over the past decades, there have been major advances in PAH treatment by developments in the field of medication targeting the major pathways in PAH pathophysiology. Moreover, new evidence underlining the multifactorial nature of the disease have fueled multiple target treatments (Maron et al., 2021). Combination treatment using two or three PAH-specific drugs seem beneficial according to recent clinical trials (Galiè, Barberà, et al., 2015; Lajoie et al., 2017; Semen \& Bast, 2019). Nutrition has also gained attention as a factor potentially influencing the progression and burden of PAH. Nutritional intervention represents a multipletarget approach by definition, as dietary components act via different mechanisms and so simultaneously modulate multiple physiological processes. Next to the work published in this thesis, other authors have recently published interesting data on the role of micronutrient deficiencies and nutritional status in the development and progression of $\mathrm{PAH}$. Omega-3 fatty acids and dietary polyphenols influence pathophysiological mechanisms such as inflammation or remodeling of the pulmonary arteries as shown in animal models of PAH (Semen \& Bast, 2019). Moreover, it was found that the gut microbiota is changed in a rat model of PAH (Callejo et al., 2018). This would merit further elucidation of the potential role of gut microbiota and its interplay with the diet, immune function and energy metabolism in the context of PAH (Callejo, Barberá, et al., 2020). As described in chapter 3 of this thesis, drugs can also influence gut microbiota, so the interplay between the gut and often used drugs in PAH might be an interesting topic for future research.

Chapter 2 of this thesis describes that the pathophysiology of PAH involves many mechanisms that may influence nutritional status and induce muscle wasting, such as inflammation, mitochondrial abnormalities, an increased energetic demand, and a decreased uptake of nutrients from the gut. The effect of certain treatment strategies, such as exercise intervention, might be limited if nutritional status is not optimal. Therefore, assessing the patient's nutritional status including screening for micronutrient deficiencies can be recommended. When indicated, nutritional interventions might help to optimize the nutritional status of the patient. However, only little is known on the nutritional status of PAH patients, the prevalence of micronutrient deficiencies and the way this may impact disease progression and quality of life. The only micronutrient that is relatively well studied is iron, since it 
is known that PAH patients often have low iron status (Rhodes, Howard, et al., 2011; Rhodes, Wharton, et al., 2011; Ruiter et al., 2011). In chapter 4 of this thesis, the prevalence of micronutrient deficiencies that are related to exercise tolerance and fatigue in PH patients is described for the first time. The results show that vitamin $\mathrm{D}$ and iron deficiency were highly prevalent in PAH and CTEPH patients, both at diagnosis and after 1,5 years of $\mathrm{PH}$ treatment. Low magnesium and phosphate levels were found in up to $21 \%$ of the patients. It was demonstrated that iron levels and transferrin saturation correlate with 6MWD, which is well in line with findings of others (Rhodes, Howard, et al., 2011; Rhodes, Wharton, et al., 2011; Ruiter et al., 2011). Vitamin D levels however did not correlate with any of the outcome parameters, therefore it remains unclear what the significance is of a vitamin D deficiency in $\mathrm{PH}$.

Other authors that reviewed studies on the presence of micronutrient deficiencies in PAH also found that iron and vitamin D deficiencies are highly prevalent in PAH patients (Callejo, Barberá, et al., 2020; Semen \& Bast, 2019). However, for vitamin D there are only a few studies available with a with a small sample size. There have been some studies on vitamin C deficiency and the development of PAH (Callejo, Barberá, et al., 2020; Kupari \& Rapola, 2012), as vitamin C impacts mechanisms involved in the PAH pathophysiology, such as the NO pathway (Taddei et al., 1998). Since a deficiency in vitamin $C$ is rare in the Western world, this has remained outside the scope of this thesis. However, for future research, it might be interesting to study whether moderate vitamin $\mathrm{C}$ deficiency plays a role in PAH. Moreover, the effect of vitamin C supplementation in PAH patients is still unclear.

Figure 6.1 depicts how both inflammation and micronutrient deficiencies have a central place in the role of nutrition in PAH treatment. This picture summarizes the main findings from this thesis. All in all, it becomes clear that more research is needed on the anti-inflammatory effect of nutritional components and the connections between micronutrient deficiencies and outcome parameters in PAH. This knowledge can then be used to start intervention studies that investigate the possible effects of supplementation strategies in $\mathrm{PAH}$ patients. Providing evidence for nutritional interventions in rare and also progressive diseases such as $\mathrm{PAH}$ however is challenging. The effect of nutritional supplementation is not always immediately clear, and this might be overshadowed by progression of the disease. 
Furthermore, getting a sufficient sample size could be difficult. Still, the potential of nutritional interventions for PAH treatment is a promising emerging field, because it can give patients a tool that actively contributes to their physical wellbeing and is also likely to generate positive psychological effects in dealing with their disease.

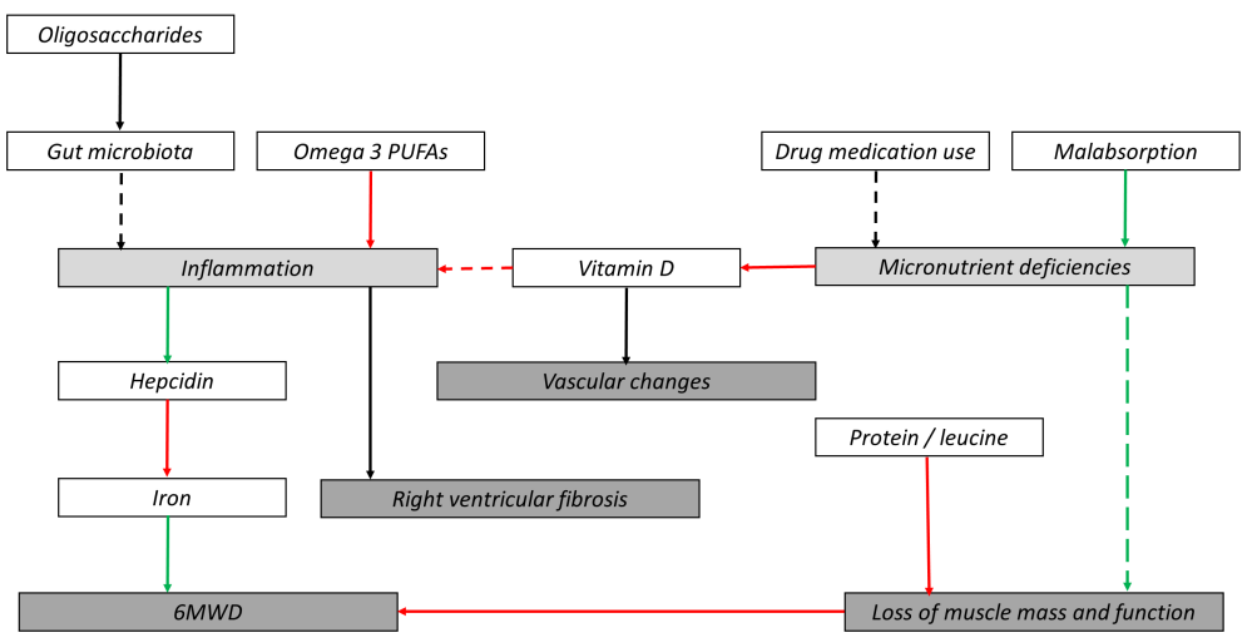

Figure 6.1: The relation of nutritional factors on the pathophysiology of PAH, as hypothesized from the results of the research described in this thesis. The effects of nutritional factors such as oligosaccharides, omega 3 PUFAs and vitamin D on the pathophysiology of PAH are mediated (at least) through inflammation and micronutrient deficiencies. Other nutritional factors, such as iron and protein/leucine exert direct effects on muscle mass and function and exercise tolerance via different mechanisms. The effects of the gut microbiota and vitamin $\mathrm{D}$ on inflammatory processes in PAH needs further investigation. Also, the effect of drug medication use on micronutrient levels and the effect of micronutrient deficiencies on muscle mass and function needs further study. Green lines indicate an increasing effect, red lines indicate a decreasing effect. Full lines indicate a known or thoroughly studied relation. Dotted lines indicate that further research is needed to confirm the hypothesized relation in PAH. 


\subsection{Medication use should be considered a potential factor influencing nutritional} status in PAH patients

PAH-specific drugs, especially those that target the prostacyclin pathway, such as selexipag, epoprostenol, iloprost and treprostinil (Del Pozo et al., 2017), are known to induce side-effects that have a negative impact on nutritional intake such as nausea, loss of appetite, vomiting, diarrhea, jaw pain and fatigue (Alami et al., 2016), as described in chapter 2. Such symptoms may lead to micronutrient deficiencies and impair nutritional status on the long term. In general, patients with chronic illness such as PAH are susceptible to develop micronutrient deficiencies (Cascino \& Hummel, 2018; Horadagoda et al., 2017; Schols, 2013; Soukoulis et al., 2009), an altered gut microbiota (Sundaram \& Fang, 2016) and muscle alterations resulting in function loss (Ebner et al., 2015; Schols et al., 2014). The use of certain commonly prescribed medications, such as proton pump inhibitors (PPIs) is also linked to these effects, as has been described in chapter 4 of this thesis. The causal relationships proposed in this chapter should however be seen as hypotheses and need further investigation.

Although research into the impact of drug use on the nutritional status of patients with PAH is still limited, it should be recognized as a potential factor influencing the physical status of the patient. Particularly in case of polypharmacy, screening for malnutrition and deficiencies in micronutrients is therefore recommended.

\subsection{Vitamin D deficiency is highly prevalent in PH patients. Investigators should broaden their field in the search for clinical implications.}

Vitamin D deficiency is highly prevalent in the general population and this has been linked to musculoskeletal, metabolic, and cardiopulmonary diseases and to disorders of the immune system (Mirdamadi \& Moshkdar, 2016; Zittermann, 2003). There is currently no consensus on the definition of vitamin $\mathrm{D}$ deficiency, optimum levels and dietary requirements (Balvers et al., 2015; Norman \& Powell, 2014). However, even when using conservative thresholds, vitamin D deficiency is a global problem. The principal causes of low 25(OH)D3 levels are inadequate sun exposure in combination with reduced dietary intake (Roth et al., 2018). It has been suggested that vitamin D influences smooth muscle cell proliferation and endothelial function, which are both affected in PAH (Atteritano et al., 2016; Demir et al., 2013; Mirdamadi 
\& Moshkdar, 2016). A relation between vitamin D levels and pulmonary function has been found, suggesting that low vitamin $D$ levels can be detrimental for patients with respiratory disease (Black \& Scragg, 2005).

In chapter 3 of this thesis, the prevalence of vitamin D deficiency is described in PAH and CTEPH patients at diagnosis and in patients that received 1,5 years of $\mathrm{PH}$ treatment. Depending on the diagnostic subclass, vitamin D deficiency occurs in up to $83 \%$ of the patients in this study. Other authors have also studied the prevalence of vitamin D deficiency in PH patients. A Japanese study concerning12 PAH and 29 CTEPH patients described that $95 \%$ of the studied population had insufficient vitamin D levels (Tanaka et al., 2017). In a Spanish cohort, 70\% of the $67 \mathrm{PAH}$ patients (idiopathic, heritable, or drug-induced) had severe vitamin D deficiency (total 25(OH)vitamin $\mathrm{D}<10 \mathrm{ng} / \mathrm{ml}$ ), with no difference between sexes. Patients with a total plasma $25(\mathrm{OH})$ vitamin D level above the median of the cohort $(7.15 \mathrm{ng} / \mathrm{ml})$ were classified as functionally better and showed higher 6MWD and better survival (Callejo, Mondejar-Parreño, et al., 2020). An Argentinian study found low 25(OH) vitamin D levels (mean: $19.25 \mathrm{ng} / \mathrm{ml}$ ) in $53 \mathrm{PH}$ patients from different $\mathrm{PH}$ subgroups. These authors also found a relation between vitamin D levels, functional class and 6MWD (Atamañuk et al., 2019). In the study described in chapter 3 of this thesis, no correlation was found between 25(OH)D levels and 6MWD or any other outcome parameter. It is possible that the power of our study was not sufficient to find an effect between vitamin D levels and outcome parameters. However, other factors might also play a role, such as the subtype classification of $\mathrm{PH}$ or the functional class of the patients included. Such data was not always available in the above cited papers.

There is hardly any data on the effect of vitamin D supplementation in PAH patients. In a small clinical study including 22 patients with pulmonary hypertension who had a vitamin D deficiency, weekly supplementation of cholecalciferol (vitamin D3) plus a daily dose of magnesium, zinc, and vitamin D for a 3-month period has been demonstrated to significantly increase the serum vitamin D level. Moreover, also the 6MWD improved. It also led to improvement in right ventricle size and function (Mirdamadi \& Moshkdar, 2016). Unfortunately, due to small sample size, a short observation period and the use of a combined intervention, it is hard to interpret the potential of correcting vitamin D deficiencies to improve the quality of life of PAH 
patients from this study alone. Two studies have tried to restore vitamin D levels in animal models of severe vitamin D deficiency. They found that serum $25(\mathrm{OH})$ vitamin D levels could be restored by supplementation (Callejo et al., 2021; Tanaka et al., 2017) and that this intervention partially improved pathological symptoms of PAH such as endothelial function (Callejo et al., 2021).

Dysregulation of cells of the innate and adaptive immune system have been found in both PAH and CTEPH patients (Heukels et al., 2021; Humbert et al., 2019; Perros et al., 2012; Quarck et al., 2015; Simonneau et al., 2017). Moreover, inflammatory and immune markers correlated with the hemodynamics and prognosis of the patient (Koudstaal, Boomars, et al., 2020). Pulmonary inflammation mediated by accumulation of macrophages near the lung arterioles is an important symptom of pulmonary remodelling in PAH (Chen et al., 2020). Another mechanism in which inflammation might play a role in PH development is by enhanced dendritic cell activation (van Uden et al., 2021). In a mouse model, this led to the development of PH and was dependent on IL-6 expression (Koudstaal, van Hulst, et al., 2020). Vitamin D has a role in immune function, since almost all immune cells express the vitamin D receptor (VDR). The effect of vitamin D on immune cells is however complex, since the expression of the VDR is different depending on the activation status (Martens et al., 2020). It can be hypothesized that preventing vitamin D deficiency could help in the treatment of immune deregulations in $\mathrm{PAH}$, although more research into this topic is clearly needed.

All in all, given the limited knowledge available, it can be concluded that the significance of a vitamin D deficiency in patients with PAH remains currently uncertain. I thereby advice that researchers broaden their field and focus on functional outcome parameters related to vitamin D deficiency such as musculoskeletal, metabolic and cardiopulmonary related outcomes. Effects of vitamin D deficiency on the immune system can also be expected and need further research. Moreover, the safety and efficacy of vitamin D supplementation in PAH patients needs investigation. 


\subsection{Reducing hepcidin levels in iron deficient PAH patients by nutritional interventions could improve the response to oral iron supplementation}

As described in chapter 3 of this thesis, our study showed that $26 \%$ of the newly diagnosed PAH patients and about $14 \%$ of the newly diagnosed CTEPH patients have anemia. Forty-two percent of the newly diagnosed PAH patients and $21 \%$ of the newly diagnosed CTEPH patients have too low plasma iron levels. In the group receiving medical treatment, about $25 \%$ of the PAH and CTEPH patients have anemia and low plasma iron levels. In general, iron deficiency was more prevalent in the PAH patients than in the CTEPH patients and more prevalent in females than in males. Low iron levels and low transferrin saturation was also linked to lower 6MWD scores in PAH and CTEPH patients in our study, which is in line with other studies (Quatredeniers et al., 2021; Sonnweber et al., 2020).

A lot of research has been done on iron deficiency in PAH patients. The relation between pulmonary hypertension, iron homeostasis and anaemia is complex. The underlying mechanisms depend on the type of $\mathrm{PH}$ and are still only partly understood. Because of this, the prevalence of iron deficiency is likely to differ between types of PH, which has consequences for treatment (Sonnweber et al., 2020). Inflammation plays an important role in (functional) iron deficiency, due to deregulation of iron homeostasis by the main regulator of iron status, hepcidin. Inflammatory cytokines lead to higher hepcidin levels and so negatively influence the uptake of iron in the gut (Kanamori et al., 2017). Because of this, there is currently no consensus on standardized diagnostic methods to assess iron deficiency in patients with diseases involving chronic inflammation. However, in order to evaluate the effect of iron supplementation strategies, this is needed (Quatredeniers et al., 2021; Sonnweber et al., 2020). Current knowledge on the effect of iron supplementation is limited, because all trials appear to be uncontrolled open-label trials in small populations combining different $\mathrm{PH}$ types, sometimes also lacking an RHC-confirmed PAH diagnosis. Moreover, iron status was assessed by different methods and secondary measurements such as plasma levels of CRP, hepcidin, vitamin D or inflammatory markers were not performed. Nevertheless, all these studies demonstrated that oral iron supplementation did not improve serum iron levels, possibly due to high hepcidin level since intravenous iron did improve iron status and also some outcome measurements (Quatredeniers et al., 2021). 
In our study described in chapter 3, newly diagnosed patients had higher hepcidin and ferritin levels than patients already receiving PH treatment. In the newly diagnosed patients showing low iron levels, mean hepcidin levels were higher than in treated patients with low iron levels. This might suggest that deregulation of iron homeostasis by high hepcidin production is a bigger problem in newly diagnosed patients compared to treated patients. From these results, it can be derived that it is worthwhile to measure hepcidin levels in patients displaying low serum iron levels. In case hepcidin levels are low, oral iron supplementation might still be effective. However, when hepcidin levels are high, intravenous iron supplementation might be indicated. However, future studies should still confirm this hypothesis.

In chapter 5, it is shown that a nutritional intervention with fish-oil and oligosaccharides had anti-inflammatory effects and positive effects on the heart and skeletal muscle in a mouse model of PAH. Whether anti-inflammatory effects of nutritional intervention can also be beneficial in counteracting the deregulation of iron homeostasis by suppressing hepcidin levels merits further study. Research in children with anemia and patients with chronic kidney disease (CKD) has shown that vitamin D can also suppress hepcidin production. In both cases, vitamin D supplementation led to a decrease of serum hepcidin concentration (Bacchetta et al., 2014; Moran-Lev et al., 2019). Given the fact that both vitamin D and iron levels were low in PAH and CTEPH patients in our study, this raises the question whether treating the vitamin D deficiency might suppress hepcidin concentration and thereby increasing iron levels or improving the response to iron supplementation in these patients.

From all of the findings described above, it seems likely that reducing hepcidin levels in iron deficient PAH patients by nutritional interventions could improve the response to oral iron supplementation. However, studies in the future should still confirm this. 


\subsection{A nutritional intervention focused on anti-inflammatory effects can suppress pathological changes in $\mathrm{PAH}$}

Chapter 5 of this thesis shows that a diet containing relatively high amounts of antiinflammatory nutritional components (fish oil and oligosaccharides) together with substances known to stimulate anabolic processes (high protein and leucine) can prevent the development of pathophysiological changes in the heart and skeletal muscle in a mouse model of PAH. Suppression of pro-inflammatory and fibrotic gene expression was part of the mechanism behind this effect. This suggests that such an intervention might have beneficial effects during the initiation and perhaps early progression of the disease. However, our data do not allow us to draw any conclusion yet regarding the effect of the intervention once the disease is progressing. To this end, further studies are warranted.

First indications that fish oil consumption can be beneficial in PAH have already been obtained by others. In a small cohort of 7 newly diagnosed PAH patients, a relative deficiency of omega 3 PUFA's (elevated omega 6 PUFA versus omega 3 PUFA ratio) was found (Semen et al., 2016). Supplementation with omega 3 PUFAs can help to restore this ratio and have anti-inflammatory effects by reduction of proinflammatory cytokines, which has been shown in a monocrotaline rat model of PAH (Morin et al., 2014) and in several other studies not specific for PAH (Semen \& Bast, 2019). Administration of omega 3 PUFAs also induced a reduction of vascular remodeling in the beforementioned rat model of PAH (Morin et al., 2014). The effects of oligosaccharides on the increased inflammatory status in PAH have not been intensively studied, but oligosaccharides have shown to induce general antiinflammatory effects via modification of the gut microbiota. In a PAH rat model, modification of the gut microbiota by antibiotics suppressed the development of PAH (Sanada et al., 2020). Unfortunately, inflammatory markers were not measured in this study. Whether anti-inflammatory effects of nutritional intervention can also be beneficial in counteracting the deregulation of iron homeostasis by suppressing hepcidin levels, such as described in chapters 2 and 4, is an interesting topic for future studies.

All in all, the research conducted in this thesis together with the knowledge from the literature suggests that nutritional intervention with anti-inflammatory components 
such as fish oil and oligosaccharides, has the potential to suppress pathological changes in PAH.

\subsection{Conclusion}

From the research summarized in this thesis, it can be concluded that PAH is a disease with multifactorial nature, which warrants multiple-target treatment. Nutritional therapy seems to have the potential to add to the effect of current conventional treatment with medication and exercise intervention, although there are still many knowledge gaps. This thesis shows new insights into the prevalence of micronutrient deficiencies in PAH and CTEPH patients. Deficiencies in iron and vitamin $\mathrm{D}$ were found to be highly prevalent both at time of diagnosis and after 1,5 years of treatment. For iron, deficiencies were clearly related to clinical and patientrelated outcome. The clinical implications of vitamin D deficiency are still unclear. Vitamin D and iron deficiency might even be interrelated via hepcidin levels and inflammatory processes, although more research regarding this subject is needed. Moreover, our research showed some first promising effects of the potential of nutritional intervention by showing prevention of pathological changes in cardiac and skeletal muscle in a PAH mouse model.

The results obtained during this project, combined with what is known from the literature, show several promising options in the field of nutrition in PAH patients. Unfortunately, only few of these measures are currently substantiated in such a way that their implementation in practice can be considered. However, the following recommendation for clinical practice can be given:

- Assessment of the nutritional status including screening for micronutrient deficiencies in PAH and CTEPH patients, especially in the case of polypharmacy.

At the same time, there are several other research directions worth exploring further. These lead to the following suggestions for a future research agenda:

- Further elucidation of the clinical implications of vitamin D deficiency in PAH and CTEPH patients. 
- The potential interrelation of vitamin $\mathrm{D}$ and iron deficiency via an effect on hepcidin levels and inflammatory processes.

- Intervention studies investigating the safety and efficacy of supplementation strategies for vitamin D in PAH and CTEPH patients.

- The effect of moderate vitamin C deficiency and the development of PAH.

- Elucidation of the potential role of the gut microbiota and its interplay with the diet, drug medication use, immune function, and energy metabolism in the context of PAH and CTEPH.

- The response to oral iron supplementation in PAH and CTEPH patients with low iron levels in combination with low versus high hepcidin levels.

- The effect of anti-inflammatory nutritional components on iron homeostasis by suppressing hepcidin levels

- The effect of nutritional intervention with anti-inflammatory and anabolic components in PAH and CTEPH once the disease has progressed. 


\section{References}

Ahn, B., Empinado, H. M., Al-Rajhi, M., Judge, A. R., \& Ferreira, L. F. (2013). Diaphragm atrophy and contractile dysfunction in a murine model of pulmonary hypertension. PloS One, 8(4), e62702.

Alami, S., Cottin, V., Mouthon, L., Desjeux, D., Quessette, E., Poiraudeau, S., \& Sitbon, O. (2016). Patients', relatives', and practitioners' views of pulmonary arterial hypertension: A qualitative study. Presse Medicale, 45(2), e11-27.

Amrein, K., Scherkl, M., Hoffmann, M., Neuwersch-Sommeregger, S., Köstenberger, M., Tmava Berisha, A., Martucci, G., Pilz, S., \& Malle, O. (2020). Vitamin D deficiency 2.0: an update on the current status worldwide. European Journal of Clinical Nutrition, 74(11), 1498-1513.

Anker, S. D., John, M., Pedersen, P. U., Raguso, C., Cicoira, M., Dardai, E., Laviano, A., Ponikowski, P., Schols, A. M. W. J., ... Vogelmeier, C. (2006). ESPEN Guidelines on Enteral Nutrition: Cardiology and Pulmonology. Clinical Nutrition, 25(2), 311-318.

Anker, S. D., Laviano, A., Filippatos, G., John, M., Paccagnella, A., Ponikowski, P., \& Schols, A. M. W. J. (2009). ESPEN Guidelines on Parenteral Nutrition: On Cardiology and Pneumology. Clinical Nutrition, 28(4), 455-460.

Anker, S. D, Ponikowski, P., Varney, S., Chua, T. P., Clark, A. L., Webb-Peploe, K. M., Harrington, D., Kox, W. J., Poole-Wilson, P. A., \& Coats, A. J. S. (1997). Wasting as independent risk factor for mortality in chronic heart failure. Lancet, 349(9058), 1050-1053.

Archer, S. L., Fang, Y.-H., Ryan, J. J., \& Piao, L. (2013). Metabolism and Bioenergetics in the Right Ventricle and Pulmonary Vasculature in Pulmonary Hypertension. Pulmonary Circulation, 3(1), 144-152.

Arena, R., Cahalin, L. P., Borghi-Silva, A., \& Myers, J. (2015). The effect of exercise training on the pulmonary arterial system in patients with pulmonary hypertension. Progress in Cardiovascular Diseases, 57(5), 480-488.

Arends, J., Bachmann, P., Baracos, V., Barthelemy, N., Bertz, H., Bozzetti, F., Fearon, K., Hütterer, E., Isenring, E., ... Preiser, J. C. (2017). ESPEN guidelines on nutrition in cancer patients. Clinical Nutrition, 36(1), 11-48.

Argilés, J. M., Busquets, S., Stemmler, B., \& López-Soriano, F. J. (2014). Cancer 
cachexia: Understanding the molecular basis. Nature Reviews Cancer, 14(11), 754-762.

Argilés, J. M., Fontes-Oliveira, C. C., Toledo, M., López-Soriano, F. J., \& Busquets, S. (2014). Cachexia: A problem of energetic inefficiency. Journal of Cachexia, Sarcopenia and Muscle, 5(4), 279-286.

Argilés, J. M., López-Soriano, F. J., \& Busquets, S. (2015). Muscle wasting in cancer: The role of mitochondria. Current Opinion in Clinical Nutrition and Metabolic Care, 18(3), 221-225.

Argilés, J. M., López-Soriano, F. J., \& Busquets, S. (2019). Mediators of cachexia in cancer patients. Nutrition, 66, 11-15.

Astrup, A., \& Bügel, S. (2019). Overfed but undernourished: recognizing nutritional inadequacies/deficiencies in patients with overweight or obesity. International Journal of Obesity, 43(2), 219-232.

Atamañuk, A. N., Litewka, D. F., Baratta, S. J., Seropian, I. M., Perez Prados, G., Payaslian, M. O., Ortiz Fragola, J. P., \& Escribano Subias, P. (2019). Vitamin D deficiency among patients with pulmonary hypertension. BMC Pulmonary Medicine, 19(1), 1-6.

Atteritano, M., Santoro, D., Corallo, G., Visalli, E., Buemi, M., Catalano, A., Lasco, A., Bitto, A., \& Squadrito, F. (2016). Skin involvement and pulmonary hypertension are associated with vitamin D insufficiency in scleroderma. International Journal of Molecular Sciences, 17(12), 2103.

Augusto, V., Padovani, C. R., Eduardo, G., \& Campos, R. (2004). SKELETAL MUSCLE FIBER TYPES IN C57BL6J MICE. Brazilian Journal of Morphological Sciences, 21(2), 89-94.

Awdish, R., Small, B., \& Cajigas, H. (2015). Development of a modified yoga program for pulmonary hypertension: A case series. Alternative Therapies in Health and Medicine, 21(2), 48-52.

Babu, A. S., Padmakumar, R., Maiya, A. G., Mohapatra, A. K., \& Kamath, R. L. (2016). Effects of Exercise Training on Exercise Capacity in Pulmonary Arterial Hypertension: A Systematic Review of Clinical Trials. Heart Lung and Circulation, 25(4), 333-341.

Bacchetta, J., Zaritsky, J. J., Sea, J. L., Chun, R. F., Lisse, T. S., Zavala, K., Nayak, A., Wesseling-Perry, K., Westerman, M., ... Hewison, M. (2014). Suppression of 
iron-regulatory hepcidin by vitamin D. Journal of the American Society of Nephrology, 25(3), 564-572.

Balanos, G. M., Dorrington, K. L., \& Robbins, P. A. (2002). Desferrioxamine elevates pulmonary vascular resistance in humans: Potential for involvement of HIF-1. Journal of Applied Physiology, 92(6), 2501-2507.

Balvers, M. G. J., Brouwer-Brolsma, E. M., Endenburg, S., De Groot, L. C. P. G. M., Kok, F. J., \& Gunnewiek, J. K. (2015). Recommended intakes of Vitamin D to optimise health, associated circulating 25-hydroxyVitamin D concentrations, and dosing regimens to treat deficiency: Workshop report and overview of current literature. Journal of Nutritional Science, 4, e23.

Baothman, O. A., Zamzami, M. A., Taher, I., Abubaker, J., \& Abu-Farha, M. (2016). The role of Gut Microbiota in the development of obesity and Diabetes. Lipids in Health and Disease, 15(1), 108.

Baracos, V. E., \& Arribas, L. (2018). Sarcopenic obesity: Hidden muscle wasting and its impact for survival and complications of cancer therapy. Annals of Oncology, 29(suppl_2), ii1-ii9.

Bauer, R., Dehnert, C., Schoene, P., Filusch, A., Bärtsch, P., Borst, M. M., Katus, H. A., \& Joachim Meyer, F. (2007). Skeletal muscle dysfunction in patients with idiopathic pulmonary arterial hypertension. Respiratory Medicine, 101(11), 2366-2369.

Becker-Grünig, T., Klose, H., Ehlken, N., Lichtblau, M., Nagel, C., Fischer, C., Gorenflo, M., Tiede, H., Schranz, D., ... Grünig, E. (2013). Efficacy of exercise training in pulmonary arterial hypertension associated with congenital heart disease. International Journal of Cardiology, 168(1), 375-381.

Benmassaoud, A., McDonald, E. G., \& Lee, T. C. (2016). Potential harms of proton pump inhibitor therapy: Rare adverse effects of commonly used drugs. CMAJ, 188(9), 657-662.

Benza, R. L., Gomberg-Maitland, M., Miller, D. P., Frost, A., Frantz, R. P., Foreman, A. J., Badesch, D. B., \& McGoon, M. D. (2012). The REVEAL registry risk score calculator in patients newly diagnosed with pulmonary arterial hypertension. Chest, 141(2), 354-362.

Benza, R. L., Miller, D. P., Barst, R. J., Badesch, D. B., Frost, A. E., \& McGoon, M. D. (2012). An evaluation of long-term survival from time of diagnosis in pulmonary arterial hypertension from the reveal registry. Chest, 142(2), 448- 
456.

Bikle, D. D. (2014). Vitamin D metabolism, mechanism of action, and clinical applications. Chemistry and Biology, 21(3), 319-329.

Bindels, L. B., Neyrinck, A. M., Loumaye, A., Catry, E., Walgrave, H., Cherbuy, C., Leclercq, S., Van Hul, M., Plovier, H., ... Delzenne, N. M. (2018). Increased gut permeability in cancer cachexia: Mechanisms and clinical relevance. Oncotarget, 9(26), 18224-18238.

Black, P. N., \& Scragg, R. (2005). Relationship between serum 25-hydroxyvitamin D and pulmonary function in the Third National Health and Nutrition Examination Survey. Chest, 128(6), 3792-3798.

Boucherie, Q., Rouby, F., Frankel, D., Roll, P., \& Micallef, J. (2018). Proton pump inhibitors prescriptions in France: Main trends from 2006 to 2016 on French health insurance database. Therapie, 73(5), 385-388.

Boucly, A., Weatherald, J., Savale, L., Jaïs, X., Cottin, V., Prevot, G., Picard, F., De Groote, P., Jevnikar, M., ... Sitbon, O. (2017). Risk assessment, prognosis and guideline implementation in pulmonary arterial hypertension. European Respiratory Journal, 50(2), 1700889.

Bouwknegt, M., van Pelt, W., Kubbinga, M. E., Weda, M., \& Havelaar, A. H. (2014). Potential association between the recent increase in campylobacteriosis incidence in the Netherlands and proton-pump inhibitor use - An ecological study. Eurosurveillance, 19(32), 20873.

Bowen, T. S., Adams, V., Werner, S., Fischer, T., Vinke, P., Brogger, M. N., Mangner, N., Linke, A., Sehr, P., ... Labeit, S. (2017). Small-molecule inhibition of MuRF1 attenuates skeletal muscle atrophy and dysfunction in cardiac cachexia. Journal of Cachexia, Sarcopenia and Muscle, 8(6), 939-953.

Bowen, T. S., Brauer, D., Rolim, N. P. L., Bækkerud, F. H., Kricke, A., Ormbostad Berre, A. M., Fischer, T., Linke, A., da Silva, G. J., ... Adams, V. (2017). Exercise training reveals inflexibility of the diaphragm in an animal model of patients with obesity-driven heart failure with a preserved ejection fraction. Journal of the American Heart Association, 6(10), e006416.

Bowen, T. S., Schuler, G., \& Adams, V. (2015). Skeletal muscle wasting in cachexia and sarcopenia: molecular pathophysiology and impact of exercise training. Journal of Cachexia, Sarcopenia and Muscle, 6(3), 197-207. 
Bray, G. A. (2000). Afferent signals regulating food intake. Proceedings of the Nutrition Society, 59(3), 373-384.

Bristow, M. R., Minobe, W., Rasmussen, R., Larrabee, P., Skerl, L., Klein, J. W., Anderson, F. L., Murray, J., Mestroni, L., ... Ginsburg, R. (1992). $\beta$-Adrenergic neuroeffector abnormalities in the failing human heart are produced by local rather than systemic mechanisms. Journal of Clinical Investigation, 89(3), 803815.

Brittain, E. L., Talati, M., Fessel, J. P., Zhu, H., Penner, N., Calcutt, M. W., West, J. D., Funke, M., Lewis, G. D., ... Hemnes, A. R. (2016). Fatty acid metabolic defects and right ventricular lipotoxicity in human pulmonary arterial hypertension. Circulation, 133(20), 1936-1944.

Buys, R., Avila, A., \& Cornelissen, V. A. (2015). Exercise training improves physical fitness in patients with pulmonary arterial hypertension: A systematic review and meta-analysis of controlled trials. BMC Pulmonary Medicine, 15(1), 40.

Cabras, P., Anedda, M., Caddeo, L., Francesco, M., \& Antonella, M. (2020). Hypomagnesemia and Hypocalcemia Caused by Proton-Pump Inhibitors Long-Term Therapy. American Journal of Therapeutics, 27(6), e676-e680.

Calder, P. C. (2015). Marine omega-3 fatty acids and inflammatory processes: Effects, mechanisms and clinical relevance. Biochimica et Biophysica Acta (BBA) Molecular and Cell Biology of Lipids, 1851(4), 469-484.

Calder, P. C. (2017). Omega-3 fatty acids and inflammatory processes: from molecules to man. Biochemical Society Transactions, 45(5), 1105-1115.

Calder, P. C., Ahluwalia, N., Brouns, F., Buetler, T., Clement, K., Cunningham, K., Esposito, K., Jönsson, L. S., Kolb, H., ... Winklhofer-Roob, B. M. (2011). Dietary factors and low-grade inflammation in relation to overweight and obesity. British Journal of Nutrition, 106(S3), S5-S78.

Calder, P. C., Bosco, N., Bourdet-Sicard, R., Capuron, L., Delzenne, N., Doré, J., Franceschi, C., Lehtinen, M. J., Recker, T., ... Visioli, F. (2017). Health relevance of the modification of low grade inflammation in ageing (inflammageing) and the role of nutrition. Ageing Research Reviews, 40, 95-119.

Callejo, M., Barberá, J. A., Duarte, J., \& Perez-Vizcaino, F. (2020). Impact of nutrition on pulmonary arterial hypertension. Nutrients, 12(1), 169.

Callejo, M., Mondejar-Parreño, G., Barreira, B., Izquierdo-Garcia, J. L., Morales- 
Cano, D., Esquivel-Ruiz, S., Moreno, L., Cogolludo, Á., Duarte, J., \& PerezVizcaino, F. (2018). Pulmonary Arterial Hypertension Affects the Rat Gut Microbiome. Scientific Reports, 8, 9681.

Callejo, M., Mondejar-Parreño, G., Esquivel-Ruiz, S., Olivencia, M. A., Moreno, L., Blanco, I., Escribano-Subias, P., Cogolludo, A., Barbera, J. A., \& Perez-Vizcaino, F. (2020). Total, Bioavailable, and Free Vitamin D Levels and Their Prognostic Value in Pulmonary Arterial Hypertension. Journal of Clinical Medicine, 9(2), 448.

Callejo, M., Morales-Cano, D., Mondejar-Parreño, G., Barreira, B., Esquivel-Ruiz, S., Olivencia, M. A., Moreno, L., Cogolludo, A., \& Perez-Vizcaino, F. (2021). Restoration of vitamin d levels improves endothelial function and increases task-like $\mathrm{k}+$ currents in pulmonary arterial hypertension associated with vitamin d deficiency. Biomolecules, 11(6), 795.

Capogrosso Sansone, A., Convertino, I., Galiulo, M. T., Salvadori, S., Pieroni, S., Knezevic, T., Mantarro, S., Marino, A., Hauben, M., ... Tuccori, M. (2017). Muscular Adverse Drug Reactions Associated with Proton Pump Inhibitors: A Disproportionality Analysis Using the Italian National Network of Pharmacovigilance Database. Drug Safety, 40(10), 895-909.

Carneiro, I. P., Mazurak, V. C., \& Prado, C. M. (2016). Clinical Implications of Sarcopenic Obesity in Cancer. Current Oncology Reports, 18(10), 62.

Carson, M. A., Reid, J., Hill, L., \& Fitzsimons, D. (2019). The need for a specific definition of cardiac cachexia. European Journal of Cardiovascular Nursing, 18(7), $524-525$.

Cascino, T. M., \& Hummel, S. L. (2018). Nutrient deficiencies in heart failure: A micro problem with macro effects? Journal of the American Heart Association, 7(17), e010447.

Casey, C., Woodside, J. V., McGinty, A., Young, I. S., McPeake, J., Chakravarthy, U., Rahu, M., Seland, J., Soubrane, G., ... Fletcher, A. E. (2019). Factors associated with serum 25-hydroxyvitamin D concentrations in older people in Europe: the EUREYE study. European Journal of Clinical Nutrition, 73(2), 319-328.

Cashman, K. D., Dowling, K. G., Škrabáková, Z., Gonzalez-Gross, M., Valtueña, J., De Henauw, S., Moreno, L., Damsgaard, C. T., Michaelsen, K. F., ... Kiely, M. (2016). Vitamin D deficiency in Europe: Pandemic? American Journal of Clinical Nutrition, 103(4), 1033-1044. 
Cernea, A., Fernández-Martínez, J. L., de Andrés-Galiana, E. J., Fernández-Muñiz, Z., Bermejo-Millo, J. C., González-Blanco, L., Solano, J. J., Abizanda, P., CotoMontes, A., \& Caballero, B. (2019). Prognostic networks for unraveling the biological mechanisms of Sarcopenia. Mechanisms of Ageing and Development, 182, 111129.

Chen, S., Yan, D., \& Qiu, A. (2020). The role of macrophages in pulmonary hypertension: Pathogenesis and targeting. International Immunopharmacology, 88,106934 .

Cheungpasitporn, W., Thongprayoon, C., Kittanamongkolchai, W., Srivali, N., Edmonds, P. J., Ungprasert, P., O'Corragain, O. A., Korpaisarn, S., \& Erickson, S. B. (2015). Proton pump inhibitors linked to hypomagnesemia: A systematic review and meta-analysis of observational studies. Renal Failure, 37(7), 12371241.

Chia, K. S. W., Wong, P. K. K., Faux, S. G., McLachlan, C. S., \& Kotlyar, E. (2017). The benefit of exercise training in pulmonary hypertension: a clinical review. Internal Medicine Journal, 47(4), 361-369.

Clark, D. W. J., \& Strandell, J. (2006). Myopathy including polymyositis: A likely class adverse effect of proton pump inhibitors? European Journal of Clinical Pharmacology, 62(6), 473-479.

Cohen, S., Nathan, J. a, \& Goldberg, A. L. (2015). Muscle wasting in disease: molecular mechanisms and promising therapies. Nature Reviews. Drug Discovery, 14(1), 58-74.

Collins, P. F., Stratton, R. J., \& Elia, M. (2012). Nutritional support in chronic obstructive pulmonary disease: a systematic review and meta-analysis. American Journal of Clinical Nutrition, 95(6), 1385-1395.

Costello, R. B., Elin, R. J., Rosanoff, A., Wallace, T. C., Guerrero-Romero, F., Hruby, A., Lutsey, P. L., Nielsen, F. H., Rodriguez-Moran, M., ... Van Horn, L. V. (2016). Perspective: The Case for an Evidence-Based Reference Interval for Serum Magnesium: The Time Has Come. Advances in Nutrition, 7(6), 977-993.

Cracowski, J.-L., Chabot, F., Labarère, J., Faure, P., Degano, B., Schwebel, C., Chaouat, A., Reynaud-Gaubert, M., Cracowski, C., ... Humbert, M. (2014). Proinflammatory cytokine levels are linked to death in pulmonary arterial hypertension. The European Respiratory Journal, 43(3), 915-917.

Csiszar, A., Labinskyy, N., Olson, S., Pinto, J. T., Gupte, S., Wu, J. M., Hu, F., Ballabh, 
P., Podlutsky, A., ... Ungvari, Z. (2009). Resveratrol prevents monocrotalineinduced pulmonary hypertension in rats. Hypertension, 54(3), 668-675.

D'Antona, G., Pellegrino, M. A., Adami, R., Rossi, R., Carlizzi, C. N., Canepari, M., Saltin, B., \& Bottinelli, R. (2003). The effect of ageing and immobilization on structure and function of human skeletal muscle fibres. The Journal of Physiology, 552(2), 499-511.

Dai, M., Wang, P., Boyd, A. D., Kostov, G., Athey, B., Jones, E. G., Bunney, W. E., Myers, R. M., Speed, T. P., ... Meng, F. (2005). Evolving gene/transcript definitions significantly alter the interpretation of GeneChip data. Nucleic Acids Research, 33(20), e175.

de Baaij, J. H. F., Hoenderop, J. G. J., \& Bindels, R. J. M. (2015). Magnesium in man: Implications for health and disease. Physiological Reviews, 95(1), 1-46.

de Jesus Perez, V. A. (2016). Molecular pathogenesis and current pathology of pulmonary hypertension. Heart Failure Reviews, 21(3), 239-257.

De Leeuw, I., Vansant, G., \& Van Gaal, L. (1992). Magnesium and obesity: influence of gender, glucose tolerance, and body fat distribution on circulating magnesium concentrations. Magnesium Research, 5(3), 183-187.

de Man, F. S., van Hees, H. W. H., Handoko, M. L., Niessen, H. W., Schalij, I., Humbert, M., Dorfmüller, P., Mercier, O., Bogaard, H.-J., ... Ottenheijm, C. A. C. (2011). Diaphragm Muscle Fiber Weakness in Pulmonary Hypertension. American Journal of Respiratory and Critical Care Medicine, 183(10), 1411-1418.

De Oliveira, A. R. S., Cruz, K. J. C., Severo, J. S., Morais, J. B. S., De Freitas, T. E. C., Araújo, R. S., \& Do Nascimento Marreiro, D. (2017). Hypomagnesemia and its relation with chronic low-grade inflammation in obesity. Revista Da Associacao Medica Brasileira, 63(2), 156-163.

Deboeck, G., Niset, G., Lamotte, M., Vachiery, J. L., Naeije, R., \& Vachie, J. (2004). Exercise testing in pulmonary arterial hypertension and in chronic heart failure. The European Respiratory Journal, 23(5), 747-751.

Del Pozo, R., Hernandez Gonzalez, I., \& Escribano-Subias, P. (2017). The prostacyclin pathway in pulmonary arterial hypertension: a clinical review. Expert Review of Respiratory Medicine, 11(6), 491-503.

DeLuca, H. F. (2004). Overview of general physiologic features and functions of vitamin D. The American Journal of Clinical Nutrition, 80(6 Suppl), 1689S-1696S. 
Demir, M., Uyan, U., Keçeoçlu, S., \& Demir, C. (2013). The Relationship between Vitamin D Deficiency and Pulmonary Hypertension. Prague Medical Report, 114(3), 154-161.

Doehner, W., Turhan, G., Leyva, F., Rauchhaus, M., Sandek, A., Jankowska, E. A., Haehling, S. Von, \& Anker, S. D. (2015). Skeletal muscle weakness is related to insulin resistance in patients with chronic heart failure. ESC Heart Failure, 2(2), 85-89.

Dolan, R. D., Almasaudi, A. S., Dieu, L. B., Horgan, P. G., McSorley, S. T., \& McMillan, D. C. (2019). The relationship between computed tomographyderived body composition, systemic inflammatory response, and survival in patients undergoing surgery for colorectal cancer. Journal of Cachexia, Sarcopenia and Muscle, 10(1), 111-122.

Dominguez, L. J., Barbagallo, M., Lauretani, F., Bandinelli, S., Bos, A., Corsi, A. M., Simonsick, E. M., \& Ferrucci, L. (2006). Magnesium and muscle performance in older persons: the InCHIANTI study. American Journal of Clinical Nutrition, 84(2), 419-426.

Don, B. R., \& Kaysen, G. (2004). Serum albumin: Relationship to inflammation and nutrition. Seminars in Dialysis, 17(6), 432-437.

Drincic, A., Fuller, E., Heaney, R. P., \& Armas, L. A. G. (2013). 25-Hydroxyvitamin D response to graded vitamin D3 supplementation among obese adults. Journal of Clinical Endocrinology and Metabolism, 98(12), 4845-4851.

Dwarkasing, J. T., Marks, D. L., Witkamp, R. F., \& Van Norren, K. (2016). Hypothalamic inflammation and food intake regulation during chronic illness. Peptides, 77, 60-66.

Dwarkasing, J. T., van Dijk, M., Dijk, F. J., Boekschoten, M. V, Faber, J., Argilès, J. M., Laviano, A., Müller, M., Witkamp, R. F., \& van Norren, K. (2014). Hypothalamic food intake regulation in a cancer-cachectic mouse model. Journal of Cachexia, Sarcopenia and Muscle, 5(2), 159-169.

Ebner, N., Sliziuk, V., Scherbakov, N., \& Sandek, A. (2015). Muscle wasting in ageing and chronic illness. ESC Heart Failure, 2(2), 58-68.

Edgar, R., Domrachev, M., \& Lash, A. E. (2002). Gene Expression Omnibus: NCBI gene expression and hybridization array data repository. Nucleic Acids Research, 30(1), 207-210. 
Evans, W. J., Morley, J. E., Argilés, J., Bales, C., Baracos, V., Guttridge, D., Jatoi, A., Kalantar-Zadeh, K., Lochs, H., ... Anker, S. D. (2008). Cachexia: A new definition. Clinical Nutrition, 27(6), 793-799.

Faber, J., Vos, P., Kegler, D., van Norren, K., Argilés, J. M., Laviano, A., Garssen, J., \& van Helvoort, A. (2008). Beneficial immune modulatory effects of a specific nutritional combination in a murine model for cancer cachexia. British Journal of Cancer, 99(12), 2029-2036.

Farhanghi, M. A., Mahboob, S., \& Ostadrahimi, A. (2009). Obesity induced magnesium deficiency can be treated by vitamin D supplementation. Journal of the Pakistan Medical Association, 59(4), 258-261.

Fearon, K. C., Voss, A. C., Hustead, D. S., \& Cancer Cachexia Study Group. (2006). Definition of cancer cachexia: effect of weight loss, reduced food intake, and systemic inflammation on functional status and prognosis. The American Journal of Clinical Nutrition, 83(6), 1345-1350.

Fernandes, R., do Rosario, V. A., Mocellin, M. C., Kuntz, M. G. F., \& Trindade, E. B. S. M. (2017). Effects of inulin-type fructans, galacto-oligosaccharides and related synbiotics on inflammatory markers in adult patients with overweight or obesity: A systematic review. Clinical Nutrition, 36(5), 1197-1206.

Freedberg, D. E., Kim, L. S., \& Yang, Y. X. (2017). The Risks and Benefits of Longterm Use of Proton Pump Inhibitors: Expert Review and Best Practice Advice From the American Gastroenterological Association. Gastroenterology, 152(4), 706-715.

Freedberg, D. E., Lebwohl, B., \& Abrams, J. A. (2014). The impact of proton pump inhibitors on the human gastrointestinal microbiome. Clinics in Laboratory Medicine, 34(4), 771-785.

Gagnon, C., Lu, Z. X., Magliano, D. J., Dunstan, D. W., Shaw, J. E., Zimmet, P. Z., Sikaris, K., Grantham, N., Ebeling, P. R., \& Daly, R. M. (2011). Serum 25hydroxyvitamin $\mathrm{D}$, calcium intake, and risk of type 2 diabetes after 5 years: Results from a national, population-based prospective study (the Australian diabetes, obesity and lifestyle study). Diabetes Care, 34(5), 1133-1138.

Galiè, N., Barberà, J. A., Frost, A. E., Ghofrani, H.-A., Hoeper, M. M., McLaughlin, V. V., Peacock, A. J., Simonneau, G., Vachiery, J.-L., ... Rubin, L. J. (2015). Initial Use of Ambrisentan plus Tadalafil in Pulmonary Arterial Hypertension. New England Journal of Medicine, 373(9), 834-844. 
Galiè, N., Humbert, M., Vachiéry, J.-L., Gibbs, S., Lang, I., Torbicki, A., Simonneau, G., Peacock, A., Vonk-Noordegraaf, A., ... Hoeper, M. (2015). 2015 ESC/ERS Guidelines for the diagnosis and treatment of pulmonary hypertension. European Heart Journal, 46(4), 903-975.

Galland, L. (2010). Diet and inflammation. Nutrition in Clinical Practice, 25(6), 634640 .

Gao, D., Trayhurn, P., \& Bing, C. (2013). 1,25-Dihydroxyvitamin D 3 inhibits the cytokine-induced secretion of MCP-1 and reduces monocyte recruitment by human preadipocytes. International Journal of Obesity, 37(3), 357-365.

Gao, H., Cheng, Y., Zong, L., Huang, L., Qiao, C., Li, W., Gong, B., Hu, J., Liu, H., ... Zhao, C. (2017). Aspirin attenuates monocrotaline-induced pulmonary arterial hypertension in rats by suppressing the ERK/MAPK pathway. Clinical and Experimental Hypertension, 39(1), 34-41.

Garcia, J. M. (2017). What is next after anamorelin? Current Opinion in Supportive and Palliative Care, 11(4), 266-271.

Garrison, S. R., Allan, G. M., Sekhon, R. K., Musini, V. M., \& Khan, K. M. (2012). Magnesium for skeletal muscle cramps. Cochrane Database of Systematic Reviews, 9, CD009402.

Gérard, P. (2016). Gut microbiota and obesity. Cellular and Molecular Life Sciences, 73(1), 147-162.

Gomez-Arroyo, J. G., Farkas, L., Alhussaini, A. A., Farkas, D., Kraskauskas, D., Voelkel, N. F., \& Bogaard, H. J. (2012). The monocrotaline model of pulmonary hypertension in perspective. American Journal of Physiology Lung Cellular and Molecular Physiology., 302(4), L363-:369.

Gommers, L. M. M., Ederveen, T. H. A., van der Wijst, J., Overmars-Bos, C., Kortman, G. A. M., Boekhorst, J., Bindels, R. J. M., de Baaij, J. H. F., \& Hoenderop, J. G. J. (2019). Low gut microbiota diversity and dietary magnesium intake are associated with the development of PPI-induced hypomagnesemia. The FASEB Journal, 33(10), 11235-11246.

Gonçalves De Carvalho, C. M. R., \& Ribeiro, S. M. L. (2017). Aging, low-grade systemic inflammation and Vitamin D: A mini-review. European Journal of Clinical Nutrition, 71(4), 434-440.

González-Saiz, L., Fiuza-Luces, C., Sanchis-Gomar, F., Santos-Lozano, A., Quezada- 
Loaiza, C. A., Flox-Camacho, A., Munguía-Izquierdo, D., Ara, I., Santalla, A., ... Lucia, A. (2017). Benefits of skeletal-muscle exercise training in pulmonary arterial hypertension: The WHOLEi+12 trial. International Journal of Cardiology, $231,277-283$.

Gröber, U., Schmidt, J., \& Kisters, K. (2020). Important drug-micronutrient interactions: A selection for clinical practice. Critical Reviews in Food Science and Nutrition, 60(2), 257-275.

Grünig, E., Ehlken, N., Ghofrani, A., Staehler, G., Meyer, F. J., Juenger, J., Opitz, C. F., Klose, H., Wilkens, H., ... Halank, M. (2011). Effect of exercise and respiratory training on clinical progression and survival in patients with severe chronic pulmonary hypertension. Respiration, 81(5), 394-401.

Grünig, E., Lichtblau, M., Ehlken, N., Ghofrani, H. A., Reichenberger, F., Staehler, G., Halank, M., Fischer, C., Seyfarth, H. J., ... Nagel, C. (2012). Safety and efficacy of exercise training in various forms of pulmonary hypertension. European Respiratory Journal, 40(1), 84-92.

Grünig, E., Maier, F., Ehlken, N., Fischer, C., Lichtblau, M., Blank, N., Fiehn, C., Stöckl, F., Prange, F., ... Nagel, C. (2012). Exercise training in pulmonary arterial hypertension associated with connective tissue diseases. Arthritis Research \& Therapy, 14(3), R148.

Guerrero-Romero, F., Flores-García, A., Saldaña-Guerrero, S., Simental-Mendía, L. E., \& Rodríguez-Morán, M. (2016). Obesity and hypomagnesemia. European Journal of Internal Medicine, 34, 29-33.

Guillot, X., Semerano, L., Saidenberg-Kermanac'h, N., Falgarone, G., \& Boissier, M. (2010). Vitamin D and inflammation. Joint Bone Spine, 77(6), 552-557.

Günther, T. (2011). Hypomagnesemia, obesity and inflammatory cytokines. Magnesium Research, 24(1), 19-20.

Hansmann, G., Wagner, R. A., Schellong, S., Perez, V. A. D. J., Urashima, T., Wang, L., Sheikh, A. Y., Suen, R. S., Stewart, D. J., \& Rabinovitch, M. (2007). Pulmonary arterial hypertension is linked to insulin resistance and reversed by peroxisome proliferator-activated receptor-gamma activation. Circulation, 115(10), 1275-1284.

Hassoun, P. M. (2014). Inflammation in pulmonary arterial hypertension: Is it time to quell the fire? European Respiratory Journal, 43(3), 685-688. 
Hess, M. W., De Baaij, J. H. F., Broekman, M., Bisseling, T. M., Haarhuis, B., Tan, A., Te Morsche, R., Hoenderop, J. G. J., Bindels, R. J. M., \& Drenth, J. P. H. (2016). Inulin significantly improves serum magnesium levels in proton pump inhibitor-induced hypomagnesaemia. Alimentary Pharmacology and Therapeutics, 43(11), 1178-1185.

Heukels, P., Corneth, O. B. J., Van Uden, D., Van Hulst, J. A. C., Van Den Toorn, L. M., Van Den Bosch, A. E., Wijsenbeek, M. S., Boomars, K. A., Kool, M., \& Hendriks, R. W. (2021). Loss of immune homeostasis in patients with idiopathic pulmonary arterial hypertension. Thorax, 215460.

Hoeper, M. M., Bogaard, H. J., Condliffe, R., Frantz, R., Khanna, D., Kurzyna, M., Langleben, D., Manes, A., Satoh, T., ... Badesch, D. B. (2013). Definitions and diagnosis of pulmonary hypertension. Journal of the American College of Cardiology, 62(25 SUPPL.), D42-D50.

Hojo, M., Asahara, T., Nagahara, A., Takeda, T., Matsumoto, K., Ueyama, H., Matsumoto, K., Asaoka, D., Takahashi, T., ... Watanabe, S. (2018). Gut Microbiota Composition Before and After Use of Proton Pump Inhibitors. Digestive Diseases and Sciences, 63(11), 2940-2949.

Holverda, S., Gan, C. T.-J., Marcus, J. T., Postmus, P. E., Boonstra, A., \& VonkNoordegraaf, A. (2006). Impaired stroke volume response to exercise in pulmonary arterial hypertension. Journal of the American College of Cardiology, 47(8), 1732-1733.

Horadagoda, C., Dinihan, T., Roberts, M., \& Kairaitis, K. (2017). Body composition and micronutrient deficiencies in patients with an acute exacerbation of chronic obstructive pulmonary disease. Internal Medicine Journal, 47(9), 10571063.

Howard, L. S. G. E., Watson, G. M. J., Wharton, J., Rhodes, C. J., Chan, K., Khengar, R., Robbins, P. A., Kiely, D. G., Condliffe, R., ... Wilkins, M. R. (2013). Supplementation of Iron in Pulmonary Hypertension: Rationale and Design of a Phase II Clinical Trial in Idiopathic Pulmonary Arterial Hypertension. Pulmonary Circulation, 3(1), 100-107.

Humbert, M., Guignabert, C., Bonnet, S., Dorfmuiller, P., Klinger, J. R., R, N. M., Olschewski, A. J., Pullamsetti, S. S., Schermuly, R. T., ... Rabinovitch, M. (2019). Pathology and pathobiology of pulmonary hypertension: state of the art and research perspectives. European Respiratory Journal, 53, 1801887.

Humbert, M., Lau, E. M. T., Montani, D., Jaïs, X., Sitbon, O., \& Simonneau, G. (2014). 
Advances in therapeutic interventions for patients with pulmonary arterial hypertension. Circulation, 130(24), 2189-2208.

Humbert, M., Morrell, N. W., Archer, S. L., Stenmark, K. R., MacLean, M. R., Lang, I. M., Christman, B. W., Weir, E. K., Eickelberg, O., .. Rabinovitch, M. (2004). Cellular and molecular pathobiology of pulmonary arterial hypertension. Journal of the American College of Cardiology, 43(12 SUPPL.), S13-S24.

Igaz, I., Simonyi, G., Balogh, S., \& Szathmári, M. (2018). Adverse effects of long-term proton-pump inhibitor therapy on adults. Orvosi Hetilap, 159(19), 735-740.

Imhann, F., Bonder, M. J., Vila, A. V., Fu, J., Mujagic, Z., Vork, L., Tigchelaar, E. F., Jankipersadsing, S. A., Cenit, M. C., ... Zhernakova, A. (2016). Proton pump inhibitors affect the gut microbiome. Gut, 65(5), 740-748.

Irizarry, R. A., Hobbs, B., Collin, F., Beazer-Barclay, Y. D., Antonellis, K. J., Scherf, U., \& Speed, T. P. (2003). Exploration, normalization, and summaries of high density oligonucleotide array probe level data. Biostatistics, 4(2), 249-264.

Janarthanan, S., Ditah, I., Adler, D. G., \& Ehrinpreis, M. N. (2012). Clostridium difficile-associated diarrhea and proton pump inhibitor therapy: A metaanalysis. American Journal of Gastroenterology, 107(7), 1001-1010.

Johansson, H. E., Zethelius, B., Öhrvall, M., Sundbom, M., \& Haenni, A. (2009). Serum magnesium status after gastric bypass surgery in obesity. Obesity Surgery, 19(9), 1250-1255.

Kahn, S. E., Hull, R. L., \& Utzschneider, K. M. (2006). Mechanisms linking obesity to insulin resistance and type 2 diabetes. Nature, 444(7121), 840-846.

Kanamori, Y., Murakami, M., Sugiyama, M., Hashimoto, O., Matsui, T., \& Funaba, M. (2017). Interleukin-1 $\beta$ (IL-1 $\beta$ ) transcriptionally activates hepcidin by inducing CCAAT enhancer-binding protein $\delta$ (C/EBPS) expression in hepatocytes. Journal of Biological Chemistry, 292(24), 10275-10287.

Karin, M., \& Lin, A. (2002). NF-kB at the crossroads of life and death. Nature Immunology, 3(3), 221-227.

Kawamoto, A., Kato, T., Minamino-Muta, E., Okano, Y., Shioi, T., \& Kimura, T. (2015). Relationships between nutritional status and markers of congestion in patients with pulmonary arterial hypertension. International Journal of Cardiology, 187(1), 27-28. 
Kinoshita, Y., Ishimura, N., \& Ishihara, S. (2018). Advantages and disadvantages of long-term proton pump inhibitor use. Journal of Neurogastroenterology and Motility, 24(2), 182-196.

Klein, G. L., Petschow, B. W., Shaw, A. L., \& Weaver, E. (2013). Gut barrier dysfunction and microbial translocation in cancer cachexia: a new therapeutic target. Current Opinion in Supportive and Palliative Care, 7(4), 361-367.

Kobayashi, H., Kato, H., Hirabayashi, Y., Murakami, H., \& Suzuki, H. (2006). Modulations of muscle protein metabolism by branched-chain amino acids in normal and muscle-atrophying rats. The Journal of Nutrition, 136(1 Suppl), 234S$236 \mathrm{~S}$.

Koudstaal, T., Boomars, K. A., \& Kool, M. (2020). Pulmonary Arterial Hypertension and Chronic Thromboembolic Pulmonary Hypertension: An Immunological Perspective. Journal of Clinical Medicine, 9(2), 561.

Koudstaal, T., van Hulst, J. A. C., Das, T., Neys, S. F. H., Merkus, D., Bergen, I. M., de Raaf, M. A., Bogaard, H. J., Boon, L., ... Hendriks, R. W. (2020). DNGR1-cremediated deletion of Tnfaip3/A20 in conventional dendritic cells induces pulmonary hypertension in mice. American Journal of Respiratory Cell and Molecular Biology, 63(5), 665-680.

Koyama, S., Hata, S., Witt, C. C., Ono, Y., Lerche, S., Ojima, K., Chiba, T., Doi, N., Kitamura, F., ... Sorimachi, H. (2008). Muscle RING-Finger Protein-1 (MuRF1) as a Connector of Muscle Energy Metabolism and Protein Synthesis. Journal of Molecular Biology, 376(5), 1224-1236.

Krause, L., Becker, M. O., Brueckner, C. S., Bellinghausen, C.-J., Becker, C., Schneider, U., Haeupl, T., Hanke, K., Hensel-Wiegel, K., ... Riemekasten, G. (2010). Nutritional status as marker for disease activity and severity predicting mortality in patients with systemic sclerosis. Annals of the Rheumatic Diseases, 69(11), 1951-1957.

Krishnan, A. V., \& Feldman, D. (2011). Mechanisms of the Anti-Cancer and AntiInflammatory Actions of Vitamin D. Annual Review of Pharmacology and Toxicology, 51(1), 311-336.

Kupari, M., \& Rapola, J. (2012). Reversible pulmonary hypertension associated with vitamin C deficiency. Chest, 142(1), 225-227.

Kurpad, A. V, \& Aeberli, I. (2012). Low serum magnesium and obesity - - Causal role or diet biomarker? Indian Pediatrics, 49(2), 100-101. 
Kurstjens, S., de Baaij, J. H. F., Overmars-Bos, C., van den Munckhof, I. C. L., Garzero, V., de Vries, M. A., Burggraaf, B., van Diepen, J. A., Riksen, N. P., ... Hoenderop, J. G. J. (2019). Increased NEFA levels reduce blood $\mathrm{Mg} 2+$ in hypertriacylglycerolaemic states via direct binding of NEFA to Mg 2+. Diabetologia, 62(2), 311-321.

Lajoie, A. C., Bonnet, S., \& Provencher, S. (2017). Combination therapy in pulmonary arterial hypertension: Recent accomplishments and future challenges. Pulmonary Circulation, 7(2), 312-325.

Lanas-Gimeno, A., Hijos, G., \& Lanas, Á. (2019). Proton pump inhibitors, adverse events and increased risk of mortality. Expert Opinion on Drug Safety, 18(11), 1043-1053.

Langhans, W., \& Hrupka, B. (1999). Interleukins and tumor necrosis factor as inhibitors of food intake. Neuropeptides, 33(5), 415-424.

Laskey, W. K., Ferrari, V. A., Palevsky, H. I., \& Kussmaul, W. G. (1993). Pulmonary artery hemodynamics in primary pulmonary hypertension. Journal of the American College of Cardiology, 21(2), 406-412.

Laveneziana, P., \& Palange, P. (2012, September 1). Physical activity, nutritional status and systemic inflammation in COPD. European Respiratory Journal, 40(3), 522-529.

Laviano, A., Koverech, A., \& Seelaender, M. (2017). Assessing pathophysiology of cancer anorexia. Current Opinion in Clinical Nutrition and Metabolic Care, 20(5), 340-345.

Le Roux, C. W., Ghatei, M. A., Gibbs, J. S. R., \& Bloom, S. R. (2005). The putative satiety hormone PYY is raised in cardiac cachexia associated with primary pulmonary hypertension. Heart, 91(2), 241-242.

Leonard, J., Marshall, J. K., \& Moayyedi, P. (2007). Systematic Review of the Risk of Enteric Infection in Patients Taking Acid Suppression. The American Journal of Gastroenterology, 102(9), 2047-2056.

Liao, S., Gan, L., \& Mei, Z. (2019). Does the use of proton pump inhibitors increase the risk of hypomagnesemia: An updated systematic review and metaanalysis. Medicine, 98(13), e15011.

Lin, K., Kools, H., de Groot, P. J., Gavai, A. K., Basnet, R. K., Cheng, F., Wu, J., Wang, X., Lommen, A., ... Leunissen, J. A. M. (2011). MADMAX - Management and 
analysis database for multiple omics experiments. Journal of Integrative Bioinformatics, 8(2), 160.

Liu, D., Yu, L., Li, S., Zhang, Q., Zhu, L., Liu, Q., Lin, H., \& Zhang, J. (2019). Association between serum magnesium and blood count: Influence of type 2 diabetes and central obesity. British Journal of Nutrition, 121(11), 1287-1293.

Liu, N., Parry, S., Xiao, Y., Zhou, S., \& Liu, Q. (2017). Molecular targets of the Warburg effect and inflammatory cytokines in the pathogenesis of pulmonary artery hypertension. Clinica Chimica Acta, 466(139), 98-104.

Loncar, G., Springer, J., Anker, M., Doehner, W., \& Lainscak, M. (2016). Cardiac cachexia: hic et nunc. Journal of Cachexia, Sarcopenia and Muscle, 7(3), 246-260.

Luttikhold, J., De Ruijter, F. M., Van Norren, K., Diamant, M., Witkamp, R. F., Van Leeuwen, P. A. M., \& Vermeulen, M. A. R. (2013). Review article: The role of gastrointestinal hormones in the treatment of delayed gastric emptying in critically ill patients. Alimentary Pharmacology and Therapeutics, 38(6), 573-583.

Macfarlane, G. T., Steed, H., \& Macfarlane, S. (2008). Bacterial metabolism and health-related effects of galacto-oligosaccharides and other prebiotics. Journal of Applied Microbiology, 104(2), 305-344.

Madonna, R., De Caterina, R., \& Geng, Y.-J. (2016). Aerobic exercise-related attenuation of arterial pulmonary hypertension: A right arrow targets the disease? Vascular Pharmacology, 87, 6-9.

Mainguy, V., Maltais, F., Saey, D., Gagnon, P., Martel, S., Simon, M., \& Provencher, S. (2010). Peripheral muscle dysfunction in idiopathic pulmonary arterial hypertension. Thorax, 65(2), 113-117.

Mak, R. H., Ikizler, A. T., Kovesdy, C. P., Raj, D. S., Stenvinkel, P., \& Kalantar-Zadeh, K. (2011). Wasting in chronic kidney disease. Journal of Cachexia, Sarcopenia and Muscle, 2(1), 9-25.

Malenfant, S., Potus, F., Mainguy, V., Leblanc, E., Malenfant, M., Ribeiro, F. Rna., Saey, D., Maltais, F., Bonnet, S., \& Provencher, S. (2015). Impaired skeletal muscle oxygenation and exercise tolerance in pulmonary hypertension. Medicine and Science in Sports and Exercise, 47(11), 2273-2282.

Manders, E., Bogaard, H.-J., Handoko, M. L., van de Veerdonk, M. C., Keogh, A., Westerhof, N., Stienen, G. J. M., Dos Remedios, C. G., Humbert, M., ... Ottenheijm, C. A. C. (2014). Contractile dysfunction of left ventricular 
cardiomyocytes in patients with pulmonary arterial hypertension. Journal of the American College of Cardiology, 64(1), 28-37.

Manders, E., Bonta, P. I., Kloek, J. J., Symersky, P., Bogaard, H.-J., Hooijman, P. E., Jasper, J. R., Malik, F. I., Stienen, G. J. M., ... Ottenheijm, C. A. C. (2016). Reduced force of diaphragm muscle fibers in patients with chronic thromboembolic pulmonary hypertension. American Journal of Physiology. Lung Cellular and Molecular Physiology, 311(1), L20-8.

Manders, E., Rain, S., Bogaard, H. J., Handoko, M. L., Stienen, G. J. M., VonkNoordegraaf, A., Ottenheijm, C. A. C., \& De Man, F. S. (2015). The striated muscles in pulmonary arterial hypertension: Adaptations beyond the right ventricle. European Respiratory Journal, 46(3), 832-842.

Mangner, N., Weikert, B., Scott Bowen, T., Sandri, M., Höllriegel, R., Erbs, S., Hambrecht, R., Schuler, G., Linke, A., ... Adams, V. (2015). Skeletal muscle alterations in chronic heart failure: Differential effects on quadriceps and diaphragm. Journal of Cachexia, Sarcopenia and Muscle, 6(4), 381-390.

Maron, B. A., Abman, S. H., Elliott, C. G., Frantz, R. P., Hopper, R. K., Horn, E. M., Nicolls, M. R., Shlobin, O. A., Shah, S. J., .. Rosenzweig, E. B. (2021). Pulmonary arterial hypertension: Diagnosis, treatment, and novel advances. American Journal of Respiratory and Critical Care Medicine, 203(12), 1472-1487.

Marra, A. M., Arcopinto, M., Bossone, E., Ehlken, N., Cittadini, A., \& Grünig, E. (2015). Pulmonary arterial hypertension-related myopathy: An overview of current data and future perspectives. Nutrition, Metabolism and Cardiovascular Diseases, 25(2), 131-139.

Martens, P., Gysemans, C., Verstuyf, A., \& Mathieu, C. (2020). Vitamin D's Effect on Immune Function. Nutrients, 12(5), 1248.

Martin, A. M., Sun, E. W., Rogers, G. B., \& Keating, D. J. (2019). The Influence of the Gut Microbiome on Host Metabolism Through the Regulation of Gut Hormone Release. Frontiers in Physiology, 10, 428.

Martin, L., Hopkins, J., Malietzis, G., Jenkins, J. T., Sawyer, M. B., Brisebois, R., MacLean, A., Nelson, G., Gramlich, L., \& Baracos, V. E. (2018). Assessment of Computed Tomography (CT)-Defined Muscle and Adipose Tissue Features in Relation to Short-Term Outcomes After Elective Surgery for Colorectal Cancer: A Multicenter Approach. Annals of Surgical Oncology, 25(9), 2669-2680.

Mateo, J., García-Lecea, M., Cadenas, S., Hernández, C., \& Moncada, S. (2003). 
Regulation of hypoxia-inducible factor-1alpha by nitric oxide through mitochondria-dependent and -independent pathways. The Biochemical Journal, 376(2), 537-544.

Mazur, A., Maier, J. A. M., Rock, E., Gueux, E., Nowacki, W., \& Rayssiguier, Y. (2007). Magnesium and the inflammatory response: Potential physiopathological implications. Archives of Biochemistry and Biophysics, 458(1), 48-56.

McDonald, E. G., Milligan, J., Frenette, C., \& Lee, T. C. (2015). Continuous Proton Pump Inhibitor Therapy and the Associated Risk of Recurrent Clostridium difficile Infection. JAMA Internal Medicine, 175(5), 784-791.

McGill, A. T., Stewart, J. M., Lithander, F. E., Strik, C. M., \& Poppitt, S. D. (2008). Relationships of low serum vitamin D 3 with anthropometry and markers of the metabolic syndrome and diabetes in overweight and obesity. Nutrition Journal, 7,4 .

McMillan, D. C. (2009). Systemic inflammation, nutritional status and survival in patients with cancer. Current Opinion in Clinical Nutrition and Metabolic Care, 12(3), 223-226.

Meyer, F. J., Lossnitzer, D., Kristen, A. V, Schoene, A. M., Kübler, W., Katus, H. A., \& Borst, M. M. (2005). Respiratory muscle dysfunction in idiopathic pulmonary arterial hypertension. The European Respiratory Journal, 25(1), 125-30.

Mirdamadi, A., \& Moshkdar, P. (2016). Benefits from the correction of vitamin D deficiency in patients with pulmonary hypertension. Caspian Journal of Internal Medicine, 7(4), 253-259.

Mohn, E., Kern, H., Saltzman, E., Mitmesser, S., \& McKay, D. (2018). Evidence of Drug-Nutrient Interactions with Chronic Use of Commonly Prescribed Medications: An Update. Pharmaceutics, 10(1), 36.

Morais, J. B. S., Severo, J. S., Santos, L. R. dos, de Sousa Melo, S. R., de Oliveira Santos, R., de Oliveira, A. R. S., Cruz, K. J. C., \& do Nascimento Marreiro, D. (2017). Role of Magnesium in Oxidative Stress in Individuals with Obesity. Biological Trace Element Research, 176(1), 20-26.

Moran-Lev, H., Galai, T., Yerushalmy-Feler, A., Weisman, Y., Anafy, A., Deutsch, V., Cipok, M., Lubetzky, R., \& Cohen, S. (2019). Vitamin D Decreases Hepcidin and Inflammatory Markers in Newly Diagnosed Inflammatory Bowel Disease Paediatric Patients: A Prospective Study. Journal of Crohn's and Colitis, 13(10), 
1287-1291.

Morin, C., Hiram, R., Rousseau, E., Blier, P. U., \& Fortin, S. (2014). Docosapentaenoic acid monoacylglyceride reduces inflammation and vascular remodeling in experimental pulmonary hypertension. American Journal of Physiology - Heart and Circulatory Physiology, 307(4), 574-586.

Morrell, J. S., Lofgren, I. E., Burke, J. D., \& Reilly, R. A. (2012). Metabolic syndrome, obesity, and related risk factors among college men and women. Journal of American College Health, 60(1), 82-89.

Mostafavi, E., Nargesi, A. A., Asbagh, F. A., Ghazizadeh, Z., Heidari, B., Mirmiranpoor, H., Esteghamati, A., Vigneron, C., \& Nakhjavani, M. (2015). Abdominal obesity and gestational diabetes: The interactive role of magnesium. Magnesium Research, 28(4), 116-125.

Muscaritoli, M., Anker, S. D., Argilés, J., Aversa, Z., Bauer, J. M., Biolo, G., Boirie, Y., Bosaeus, I., Cederholm, T., ... Sieber, C. C. (2010). Consensus definition of sarcopenia, cachexia and pre-cachexia: Joint document elaborated by Special Interest Groups (SIG) ' cachexia-anorexia in chronic wasting diseases' and ' nutrition in geriatrics'. Clinical Nutrition, 29(2), 154-159.

Naderi, N., Boobejame, P., Bakhshandeh, H., Amin, A., Taghavi, S., \& Maleki, M. (2014). Insulin Resistance in Pulmonary Arterial Hypertension, Is it a Novel Disease Modifier? Research in Cardiovascular Medicine, 3(3), e19710.

Nielsen, F. H. (2010). Magnesium, inflammation, and obesity in chronic disease. Nutrition Reviews, 68(6), 333-340.

Noguchi, Y., Yoshikawa, T., Matsumoto, A., Svaninger, G., \& Gelin, J. (1996). Are cytokines possible mediators of cancer cachexia? Surgery Today, 26(7), 467-475.

Nootens, M., Wolfkiel, C. J., Chomka, E. V, \& Rich, S. (1995). Understanding Right and left Ventricular Systolic Function and Interactions at Rest and With Exercise in Primary Pulmonary HyPertension. Am J Cardiol, 75, 374-377.

Norman, P. E., \& Powell, J. T. (2014). Vitamin D and cardiovascular disease. Circulation Research, 114(2), 379-393.

Pachikian, B. D., Neyrinck, A. M., Deldicque, L., De Backer, F. C., Catry, E., Dewulf, E. M., Sohet, F. M., Bindels, L. B., Everard, A., ... Delzenne, N. M. (2010). Changes in Intestinal Bifidobacteria Levels Are Associated with the Inflammatory Response in Magnesium-Deficient Mice. The Journal of Nutrition, 
140(3), 509-514.

Paffett, M. L., Lucas, S. N., \& Campen, M. J. (2012). Resveratrol reverses monocrotaline-induced pulmonary vascular and cardiac dysfunction: A potential role for atrogin-1 in smooth muscle. Vascular Pharmacology, 56(1-2), 64-73.

Pandey, A., Garg, S., Khunger, M., Garg, S., Kumbhani, D. J., Chin, K. M., \& Berry, J. D. (2015). Efficacy and Safety of Exercise Training in Chronic Pulmonary Hypertension: Systematic Review and Meta-Analysis. Circulation: Heart Failure, 8(6), 1032-1043.

Park, C. H., Kim, E. H., Roh, Y. H., Kim, H. Y., \& Lee, S. K. (2014). The Association between the Use of Proton Pump Inhibitors and the Risk of Hypomagnesemia: A Systematic Review and Meta-Analysis. PLoS ONE, 9(11), e112558.

Parsons, C., Johnston, S., Mathie, E., Baron, N., MacHen, I., Amador, S., \& Goodman, C. (2012). Potentially inappropriate prescribing in older people with dementia in care homes: A retrospective analysis. Drugs Aging, 29(2), 143-155.

Passino, C., Barison, A., Vergaro, G., Gabutti, A., Borrelli, C., Emdin, M., \& Clerico, A. (2015). Markers of fibrosis, inflammation, and remodeling pathways in heart failure. Clinica Chimica Acta, 443, 29-38.

Paulin, R., \& Michelakis, E. D. (2014). The metabolic theory of pulmonary arterial hypertension. Circulation Research, 115(1), 148-164.

Peng, H., Xiao, Y., Deng, X., Luo, J., Hong, C., \& Qin, X. (2016). The Warburg effect: A new story in pulmonary arterial hypertension. Clinica Chimica Acta, 461, 5358.

Pereira-Santos, M., Costa, P. R. F., Assis, A. M. O., Santos, C. A. S. T., \& Santos, D. B. (2015). Obesity and vitamin D deficiency: A systematic review and metaanalysis. Obesity Reviews, 16(4), 341-349.

Perros, F., Dorfmüller, P., Montani, D., Hammad, H., Waelput, W., Girerd, B., Raymond, N., Mercier, O., Mussot, S., ... Lambrecht, B. N. (2012). Pulmonary lymphoid neogenesis in idiopathic pulmonary arterial hypertension. American Journal of Respiratory and Critical Care Medicine, 185(3), 311-321.

Poehlman, E. T. (1994). Increased Resting Metabolic Rate in Patients with Congestive Heart Failure. Annals of Internal Medicine, 121(11), 860-862. 
Ponikowski, P., Voors, A. A., Anker, S. D., Bueno, H., Cleland, J. G. F., Coats, A. J. S., Falk, V., González-Juanatey, J. R., Harjola, V. P., ... Van Der Meer, P. (2016). 2016 ESC Guidelines for the diagnosis and treatment of acute and chronic heart failure. European Heart Journal, 37(27), 2129-2200.

Price, L. C., Wort, S. J., Perros, F., Dorfmüller, P., Huertas, A., Montani, D., CohenKaminsky, S., \& Humbert, M. (2012). Inflammation in Pulmonary Arterial Hypertension. Chest, 141(1), 210-221.

Provencher, S., Chemla, D., Hervé, P., Sitbon, O., Humbert, M., \& Simonneau, G. (2006). Heart rate responses during the 6-minute walk test in pulmonary arterial hypertension. The European Respiratory Journal: Official Journal of the European Society for Clinical Respiratory Physiology, 27(1), 114-120.

Pugh, M. E., Buchowski, M. S., Robbins, I. M., Newman, J. H., \& Hemnes, A. R. (2012). Physical activity limitation as measured by accelerometry in pulmonary arterial hypertension. Chest, 142(6), 1391-1398.

Pugh, M. E., Robbins, I. M., Rice, T. W., West, J., Newman, J. H., \& Hemnes, A. R. (2011). Unrecognized glucose intolerance is common in pulmonary arterial hypertension. Journal of Heart and Lung Transplantation, 30(8), 904-911.

Pullamsetti, S. S., Savai, R., Janssen, W., Dahal, B. K., Seeger, W., Grimminger, F., Ghofrani, H. A., Weissmann, N., \& Schermuly, R. T. (2011). Inflammation, immunological reaction and role of infection in pulmonary hypertension. Clinical Microbiology and Infection, 17(1), 7-14.

Pulmonary Hypertension Association. (2008). Recommendations for Exercise in Patients with PAH. Retrieved 15 May 2018, from https://phassociation.org/medicalprofessionals/consensusstatements/exercise/

Quadery, S. R., Swift, A. J., Billings, C. G., Thompson, A. A. R., Elliot, C. A., Hurdman, J., Charalampopoulos, A., Sabroe, I., Armstrong, I. J., ... Kiely, D. G. (2018). The impact of patient choice on survival in chronic thromboembolic pulmonary hypertension. European Respiratory Journal, 52(3), 1800589.

Quarck, R., Wynants, M., Verbeken, E., Meyns, B., \& Delcroix, M. (2015). Contribution of inflammation and impaired angiogenesis to the pathobiology of chronic thromboembolic pulmonary hypertension. European Respiratory Journal, 46(2), 431-443.

Quatredeniers, M., Montani, D., Cohen-Solal, A., \& Perros, F. (2021). Iron deficiency in pulmonary arterial hypertension: perspectives. Pulmonary Circulation, 11(3). 
Rabinovitch, M., Guignabert, C., Humbert, M., \& Nicolls, M. R. (2014). Inflammation and immunity in the pathogenesis of pulmonary arterial hypertension. Circulation Research, 115(1), 165-175.

Rådegran, G., Kjellström, B., Ekmehag, B., Larsen, F., Rundqvist, B., Blomquist, S. B., Gustafsson, C., Hesselstrand, R., Karlsson, M., ... Jansson, K. (2016). Characteristics and survival of adult Swedish PAH and CTEPH patients 20002014. Scandinavian Cardiovascular Journal, 50(4), 243-250.

Ramakrishnan, L., Pedersen, S. L., Toe, Q. K., Quinlan, G. J., \& Wort, S. J. (2018). Pulmonary arterial hypertension: Iron matters. Frontiers in Physiology, 9, 641.

Rayssiguier, Y., Libako, P., Nowacki, W., \& Rock, E. (2010). Magnesium deficiency and metabolic syndrome: Stress and inflammation may reflect calcium activation. Magnesium Research, 23(2), 73-80.

Resnick, L. M. (1992). Cellular ions in hypertension, insulin resistance, obesity, and diabetes: A unifying theme. Journal of the American Society of Nephrology, 3(4 SUPPL.), S78-85.

Rhodes, C. J., Howard, L. S., Busbridge, M., Ashby, D., Kondili, E., Gibbs, J. S. R., Wharton, J., \& Wilkins, M. R. (2011). Iron deficiency and raised hepcidin in idiopathic pulmonary arterial hypertension. Journal of the American College of Cardiology, 58(3), 300-309.

Rhodes, C. J., Wharton, J., Howard, L., Gibbs, J. S. R., Vonk-Noordegraaf, A., \& Wilkins, M. R. (2011). Iron deficiency in pulmonary arterial hypertension: A potential therapeutic target. European Respiratory Journal, 38(6), 1453-1460.

Riley, M. S., Pórszász, J., Engelen, M. P. K. J., Brundage, B. H., \& Wasserman, K. (2000). Gas exchange responses to continuous incremental cycle ergometry exercise in primary pulmonary hypertension in humans. European Journal of Applied Physiology, 83(1), 63-70.

Risco, F., \& Traba, M. L. (1992). Influence of magnesium on the in vitro synthesis of 24,25-dihydroxyvitamin D3 and 1 alpha, 25-dihydroxyvitamin D3. Magnesium Research, 5(1), 5-14.

Ritchie, M. E., Phipson, B., Wu, D., Hu, Y., Law, C. W., Shi, W., \& Smyth, G. K. (2015). Limma powers differential expression analyses for RNA-sequencing and microarray studies. Nucleic Acids Research, 43(7), e47.

Robinson, J. C., Graham, B. B., Rouault, T. C., \& Tuder, R. M. (2014). The crossroads 
of iron with hypoxia and cellular metabolism: Implications in the pathobiology of pulmonary hypertension. American Journal of Respiratory Cell and Molecular Biology, 51(6), 721-729.

Romanello, V., \& Sandri, M. (2016). Mitochondrial quality control and muscle mass maintenance. Frontiers in Physiology, 6, 422.

Rosca, M. G., \& Hoppel, C. L. (2013). Mitochondrial dysfunction in heart failure. Heart Failure Reviews, 18(5), 607-622.

Roth, D. E., Abrams, S. A., Aloia, J., Bergeron, G., Bourassa, M. W., Brown, K. H., Calvo, M. S., Cashman, K. D., Combs, G., ... Whiting, S. J. (2018). Global prevalence and disease burden of vitamin $\mathrm{D}$ deficiency: a roadmap for action in low- and middle-income countries. Annals of the New York Academy of Sciences, 1430, 44-79.

Ruiter, G., Lankhorst, S., Boonstra, A., Postmus, P. E., Zweegman, S., Westerhof, N., Van Der Laarse, W. J., \& Vonk-Noordegraaf, A. (2011). Iron deficiency is common in idiopathic pulmonary arterial hypertension. European Respiratory Journal, 37(6), 1386-1391.

Ruiter, G., Manders, E., Happé, C. M., Schalij, I., Groepenhoff, H., Howard, L. S., Wilkins, M. R., Bogaard, H. J., Westerhof, N., ... Vonk-Noordegraaf, A. (2015). Intravenous iron therapy in patients with idiopathic pulmonary arterial hypertension and iron deficiency. Pulmonary Circulation, 5(3), 466-472.

Ryan, J. J., \& Archer, S. L. (2014). The right ventricle in pulmonary arterial hypertension: Disorders of metabolism, angiogenesis and adrenergic signaling in right ventricular failure. Circulation Research, 115(1), 176-188.

Samson, N., \& Paulin, R. (2017). Epigenetics, inflammation and metabolism in right heart failure associated with pulmonary hypertension. Pulmonary Circulation, $7(3), 572-587$.

Sanada, T. J., Hosomi, K., Shoji, H., Park, J., Naito, A., Ikubo, Y., Yanagisawa, A., Kobayashi, T., Miwa, H., ... Tatsumi, K. (2020). Gut microbiota modification suppresses the development of pulmonary arterial hypertension in an SU5416/hypoxia rat model. Pulmonary Circulation, 10(3), 2045894020929147.

Sanders, K. J. C., Kneppers, A. E. M., van de Bool, C., Langen, R. C. J., \& Schols, A. M. W. J. (2016). Cachexia in chronic obstructive pulmonary disease: New insights and therapeutic perspective. Journal of Cachexia, Sarcopenia and Muscle, $7(1), 5-22$. 
Sandqvist, A., Kylhammar, D., Bartfay, S. E., Hesselstrand, R., Hjalmarsson, C., Kavianipour, M., Nisell, M., Rådegran, G., Wikström, G., ... Söderberg, S. (2021). Risk stratification in chronic thromboembolic pulmonary hypertension predicts survival. Scandinavian Cardiovascular Journal, 55(1), 43-49.

Sartor, M. A., Tomlinson, C. R., Wesselkamper, S. C., Sivaganesan, S., Leikauf, G. D., $\&$ Medvedovic, M. (2006). Intensity-based hierarchical Bayes method improves testing for differentially expressed genes in microarray experiments. $B M C$ Bioinformatics, 7(1), 538.

Sassi, F., Tamone, C., \& D'amelio, P. (2018). Vitamin D: Nutrient, hormone, and immunomodulator. Nutrients, 10(11), 1656.

Scarpignato, C., Gatta, L., Zullo, A., \& Blandizzi, C. (2016). Effective and safe proton pump inhibitor therapy in acid-related diseases - A position paper addressing benefits and potential harms of acid suppression. BMC Medicine, 14(1), 179.

Scherbakov, N., \& Doehner, W. (2019). Cachexia as a common characteristic in multiple chronic disease. Journal of Cachexia, Sarcopenia and Muscle, 9(7), 11891191.

Schols, A. M., Ferreira, I. M., Franssen, F. M., Gosker, H. R., Janssens, W., Muscaritoli, M., Pison, C., Rutten-Van Mölken, M., Slinde, F., ... Singh, S. J. (2014). Nutritional assessment and therapy in COPD: A European respiratory society statement. European Respiratory Journal, 44(6), 1504-1520.

Schols, A. M. W. J. (2013). Nutrition as a metabolic modulator in COPD. Chest, 144(4), 1340-1345.

Semen, K. O., \& Bast, A. (2019). Towards improved pharmacotherapy in pulmonary arterial hypertension. Can diet play a role? Clinical Nutrition ESPEN, 30, 159169.

Semen, K., Yelisyeyeva, O., Jarocka-Karpowicz, I., Kaminskyy, D., Solovey, L., Skrzydlewska, E., \& Yavorskyi, O. (2016). Sildenafil reduces signs of oxidative stress in pulmonary arterial hypertension: Evaluation by fatty acid composition, level of hydroxynonenal and heart rate variability. Redox Biology, $7,48-57$.

Shahi, A., Aslani, S., Ataollahi, M. R., \& Mahmoudi, M. (2019). The role of magnesium in different inflammatory diseases. Inflammopharmacology, 27(4), 649-661. 
Sheen, E., \& Triadafilopoulos, G. (2011). Adverse effects of long-term proton pump inhibitor therapy. Digestive Diseases and Sciences, 56(4), 931-950.

Shen, L., Shen, J., Pu, J., \& He, B. (2011). Aspirin Attenuates Pulmonary Arterial Hypertension in Rats by Reducing Plasma 5-Hydroxytryptamine Levels. Cell Biochemistry and Biophysics, 61(1), 23-31.

Shin, J. M., \& Sachs, G. (2008). Pharmacology of proton pump inhibitors. Current Gastroenterology Reports, 10(6), 528-534.

Simonneau, G., Montani, D., Celermajer, D. S., Denton, C. P., Gatzoulis, M. A., Krowka, M., Williams, P. G., \& Souza, R. (2019). Haemodynamic definitions and updated clinical classification of pulmonary hypertension. The European Respiratory Journal, 53, 1801913.

Simonneau, G., Torbicki, A., Dorfmüller, P., \& Kim, N. (2017). The pathophysiology of chronic thromboembolic pulmonary hypertension. European Respiratory Review, 26(143), 160112.

Slusher, A. L., McAllister, M. J., \& Huang, C. J. (2015). A therapeutic role for vitamin $\mathrm{D}$ on obesity-associated inflammation and weight-loss intervention. Inflammation Research, 64(8), 565-575.

Smith, T. G., Balanos, G. M., Croft, Q. P. P., Talbot, N. P., Dorrington, K. L., Ratcliffe, P. J., \& Robbins, P. A. (2008). The increase in pulmonary arterial pressure caused by hypoxia depends on iron status. The Journal of Physiology, 586(24), 5999-6005.

Society on Sarcopenia Cachexia and Wasting Disorders. (2018). Definition of Cachexia and Sarcopenia. Retrieved 15 May 2018, from http://societyscwd.org/cachexia-definition/

Sonnweber, T., Pizzini, A., Tancevski, I., Löffler-Ragg, J., \& Weiss, G. (2020). Anaemia, iron homeostasis and pulmonary hypertension: a review. Internal and Emergency Medicine, 15(4), 573-585.

Soon, E., Treacy, C. M., Toshner, M. R., MacKenzie-Ross, R., Manglam, V., Busbridge, M., Sinclair-McGarvie, M., Arnold, J., Sheares, K. K., ... PepkeZaba, J. (2011). Unexplained iron deficiency in idiopathic and heritable pulmonary arterial hypertension. Thorax, 66(4), 326-32.

Soukoulis, V., Dihu, J. B., Sole, M., Anker, S. D., Cleland, J., Fonarow, G. C., Metra, M., Pasini, E., Strzelczyk, T., ... Gheorghiade, M. (2009). Micronutrient 
Deficiencies. An Unmet Need in Heart Failure. Journal of the American College of Cardiology, 54(18), 1660-1673.

Sousa-Santos, A. R., Afonso, C., Santos, A., Borges, N., Moreira, P., Padrão, P., Fonseca, I., \& Amaral, T. F. (2018). The association between 25(OH)D levels, frailty status and obesity indices in older adults. PLOS ONE, 13, e0198650.

Standley, R. A., Liu, S. Z., Jemiolo, B., Trappe, S. W., \& Trappe, T. A. (2013). Prostaglandin E2 induces transcription of skeletal muscle mass regulators interleukin-6 and muscle RING finger-1 in humans. Prostaglandins Leukotrienes and Essential Fatty Acids, 88(5), 361-364.

Stokić, E., Kupusinac, A., Tomic-Naglic, D., Smiljenic, D., Kovacev-Zavisic, B., SrdicGalic, B., Soskic, S., \& Isenovic, E. R. (2015). Vitamin D and Dysfunctional Adipose Tissue in Obesity. Angiology, 66(7), 613-618.

Strand, D. S., Kim, D., \& Peura, D. A. (2017). 25 years of proton pump inhibitors: A comprehensive review. Gut and Liver, 11(1), 27-37.

Su, V. Y. F., Liao, H. F., Perng, D. W., Chou, Y. C., Hsu, C. C., Chou, C. L., Chang, Y. L., Yen, J. C., Chen, T. J., \& Chou, T. C. (2018). Proton pump inhibitors use is associated with a lower risk of acute exacerbation and mortality in patients with coexistent COPD and GERD. International Journal of COPD, 13, 2907-2915.

Subramanian, A., Tamayo, P., Mootha, V. K., Mukherjee, S., Ebert, B. L., Gillette, M. A., Paulovich, A., Pomeroy, S. L., Golub, T. R., ... Mesirov, J. P. (2005). Gene set enrichment analysis: A knowledge-based approach for interpreting genomewide expression profiles. Proceedings of the National Academy of Sciences, 102(43), 15545-15550.

Sun, L., Cai, Z., Pu, J., Li, J., Shen, J., Yang, C., \& He, B. (2017). 5-Aminosalicylic Acid Attenuates Monocrotaline-Induced Pulmonary Arterial Hypertension in Rats by Increasing the Expression of Nur77. Inflammation, 40(3), 806-817.

Sun, X. G., Hansen, J. E., Oudiz, R. J., \& Wasserman, K. (2001). Exercise Pathophysiology in Patients With Primary Pulmonary Hypertension. Circulation, 104(4), 429-435.

Sundaram, V., \& Fang, J. C. (2016). Gastrointestinal and Liver Issues in Heart Failure. Circulation, 133(17), 1696-1703.

Sutendra, G., \& Michelakis, E. D. (2014). The metabolic basis of pulmonary arterial hypertension. Cell Metabolism, 19(4), 558-573. 
Taddei, S., Virdis, A., Ghiadoni, L., Magagna, A., \& Salvetti, A. (1998). Vitamin C improves endothelium-dependent vasodilation by restoring nitric oxide activity in essential hypertension. Circulation, 97(22), 2222-2229.

Talati, M. H., Brittain, E. L., Fessel, J. P., Penner, N., Atkinson, J., Funke, M., Grueter, C., Jerome, W. G., Freeman, M., ... Hemnes, A. R. (2016). Mechanisms of Lipid Accumulation in the Bone Morphogenetic Protein Receptor Type 2 Mutant Right Ventricle. American Journal of Respiratory and Critical Care Medicine, 194(6), 719-728.

Talati, M., \& Hemnes, A. (2015). Fatty acid metabolism in pulmonary arterial hypertension: role in right ventricular dysfunction and hypertrophy. Pulmonary Circulation, 5(2), 269-278.

Tan, B. H. L., Birdsell, L. A., Martin, L., Baracos, V. E., \& Fearon, K. C. H. (2009). Sarcopenia in an overweight or obese patient is an adverse prognostic factor in pancreatic cancer. Clinical Cancer Research, 15(22), 6973-6979.

Tanada, Y., Shioi, T., Kato, T., Kawamoto, A., Okuda, J., \& Kimura, T. (2015). Branched-chain amino acids ameliorate heart failure with cardiac cachexia in rats. Life Sciences, 137, 20-27.

Tanaka, H., Kataoka, M., Isobe, S., Yamamoto, T., Shirakawa, K., Endo, J., Satoh, T., Hakamata, Y., Kobayashi, E., ... Fukuda, K. (2017). Therapeutic impact of dietary vitamin $\mathrm{D}$ supplementation for preventing right ventricular remodeling and improving survival in pulmonary hypertension. PLoS ONE, 12(7), e0180615.

ten Haaf, D. S. M., Balvers, M. G. J., Timmers, S., Eijsvogels, T. M. H., Hopman, M. T. E., \& Klein Gunnewiek, J. M. T. (2019). Determinants of vitamin D status in physically active elderly in the Netherlands. European Journal of Nutrition, 58(8), 3121-3128.

ter Borg, S., de Groot, L. C. P. G. M., Mijnarends, D. M., de Vries, J. H. M., Verlaan, S., Meijboom, S., Luiking, Y. C., \& Schols, J. M. G. A. (2016). Differences in Nutrient Intake and Biochemical Nutrient Status Between Sarcopenic and Nonsarcopenic Older Adults-Results From the Maastricht Sarcopenia Study. Journal of the American Medical Directors Association, 17(5), 393-401.

Tessier, A. J., Wing, S. S., Rahme, E., Morais, J. A., \& Chevalier, S. (2019). Physical function-derived cut-points for the diagnosis of sarcopenia and dynapenia from the Canadian longitudinal study on aging. Journal of Cachexia, Sarcopenia and Muscle, 10(5), 985-999. 
The Criteria Committee of the New York Heart Association (1994). Nomenclature and Criteria for Diagnosis of Diseases of the Heart and Great Vessels. (9th ed.). Boston, Mass.: Little, Brown \& Co.

Thongon, N., \& Krishnamra, N. (2012). Apical acidity decreases inhibitory effect of omeprazole on $\mathrm{Mg}(2+)$ absorption and claudin-7 and -12 expression in Caco-2 monolayers. Experimental and Molecular Medicine, 44(11), 684-693.

Toh, J. W. T., Ong, E., \& Wilson, R. (2015). Hypomagnesaemia associated with longterm use of proton pump inhibitors. Gastroenterology Report, 3(3), 243-253.

Tonelli, A. R., Plana, J. C., Heresi, G. A., \& Dweik, R. A. (2012). Prevalence and prognostic value of left ventricular diastolic dysfunction in idiopathic and heritable pulmonary arterial hypertension. Chest, 141(6), 1457-1465.

Tran, D. L., Lau, E. M. T., Celermajer, D. S., Davis, G. M., \& Cordina, R. (2018). Pathophysiology of exercise intolerance in pulmonary arterial hypertension. Respirology, 23(2), 148-159.

Ulrich, S., Fischler, M., Speich, R., \& Bloch, K. E. (2013). Wrist actigraphy predicts outcome in patients with pulmonary hypertension. Respiration, 86(1), 45-51.

Uwitonze, A. M., \& Razzaque, M. S. (2018). Role of Magnesium in Vitamin D Activation and Function. The Journal of the American Osteopathic Association, 118(3), 181-189.

Vaezi, M. F., Yang, Y. X., \& Howden, C. W. (2017). Complications of Proton Pump Inhibitor Therapy. Gastroenterology, 153(1), 35-48.

Valentova, M., Von Haehling, S., Krause, C., Ebner, N., Steinbeck, L., Cramer, L., Doehner, W., Murin, J., Anker, S. D., \& Sandek, A. (2013). Cardiac cachexia is associated with right ventricular failure and liver dysfunction. International Journal of Cardiology, 169(3), 219-224.

van Dam, R. M., Snijder, M. B., Dekker, J. M., Stehouwer, C. D. A., Bouter, L. M., Heine, R. J., \& Lips, P. (2007). Potentially modifiable determinants of vitamin D status in an older population in the Netherlands: The Hoorn Study. American Journal of Clinical Nutrition, 85(3), 755-761.

van der Ende, M., Grefte, S., Plas, R., Meijerink, J., Witkamp, R. F., Keijer, J., \& van Norren, K. (2018). Mitochondrial dynamics in cancer-induced cachexia. Biochimica et Biophysica Acta - Reviews on Cancer, 1870(2), 137-150. 
van Dijk, M., Dijk, F. J., Hartog, A., van Norren, K., Verlaan, S., van Helvoort, A., Jaspers, R. T., \& Luiking, Y. (2018). Reduced dietary intake of micronutrients with antioxidant properties negatively impacts muscle health in aged mice. Journal of Cachexia, Sarcopenia and Muscle, 9(1), 146-159.

van Dronkelaar, C., van Velzen, A., Abdelrazek, M., van der Steen, A., Weijs, P. J. M., \& Tieland, M. (2018). Minerals and Sarcopenia; The Role of Calcium, Iron, Magnesium, Phosphorus, Potassium, Selenium, Sodium, and Zinc on Muscle Mass, Muscle Strength, and Physical Performance in Older Adults: A Systematic Review. Journal of the American Medical Directors Association, 19(1), 6-11.

van Empel, V. P. M., Lee, J., Williams, T. J., \& Kaye, D. M. (2014). Iron deficiency in patients with idiopathic pulmonary arterial hypertension. Heart Lung and Circulation, 23(3), 287-292.

van Norren, K., Dwarkasing, J. T., \& Witkamp, R. F. (2017). The role of hypothalamic inflammation, the hypothalamic-pituitary-Adrenal axis and serotonin in the cancer anorexia-cachexia syndrome. Current Opinion in Clinical Nutrition and Metabolic Care, 20(5), 396-401.

van Norren, K., Kegler, D., Argilés, J. M., Luiking, Y., Gorselink, M., Laviano, a, Arts, K., Faber, J., Jansen, H., ... van Helvoort, a. (2009). Dietary supplementation with a specific combination of high protein, leucine, and fish oil improves muscle function and daily activity in tumour-bearing cachectic mice. British Journal of Cancer, 100(5), 713-722.

van Orten-Luiten, A. C. B., Janse, A., Verspoor, E., Brouwer-Brolsma, E. M., \& Witkamp, R. F. (2018). Drug use is associated with lower plasma magnesium levels in geriatric outpatients; possible clinical relevance. Clinical Nutrition, 38, 2668-2676.

van Uden, D., Koudstaal, T., van Hulst, J. A. C., Bergen, I. M., Gootjes, C., Morrell, N. W., van Loo, G., von der Thüsen, J. H., van den Bosch, T. P. P., ... Hendriks, R. W. (2021). Central role of dendritic cells in pulmonary arterial hypertension in human and mice. International Journal of Molecular Sciences, 22(4), 1-19.

Varian, B. J., Goureshetti, S., Poutahidis, T., Lakritz, J. R., Levkovich, T., Kwok, C., Teliousis, K., Ibrahim, Y. M., Mirabal, S., \& Erdman, S. E. (2016). Beneficial bacteria inhibit cachexia. Oncotarget, 7(11), 11803-11816.

Vecchié, A., Dallegri, F., Carbone, F., Bonaventura, A., Liberale, L., Portincasa, P., Frühbeck, G., \& Montecucco, F. (2018). Obesity phenotypes and their 
paradoxical association with cardiovascular diseases. European Journal of Internal Medicine, 48, 6-17.

Veith, C., Schermuly, R. T., Brandes, R. P., \& Weissmann, N. (2016). Molecular mechanisms of hypoxia-inducible factor-induced pulmonary arterial smooth muscle cell alterations in pulmonary hypertension. Journal of Physiology, 594(5), 1167-1177.

Verlaan, S., Aspray, T. J., Bauer, J. M., Cederholm, T., Hemsworth, J., Hill, T. R., McPhee, J. S., Piasecki, M., Seal, C., ... Brandt, K. (2017). Nutritional status, body composition, and quality of life in community-dwelling sarcopenic and non-sarcopenic older adults: A case-control study. Clinical Nutrition, 36(1), 267274.

Veronese, N., Berton, L., Carraro, S., Bolzetta, F., De Rui, M., Perissinotto, E., Toffanello, E. D., Bano, G., Pizzato, S., ... Manzato, E. (2014). Effect of oral magnesium supplementation on physical performance in healthy elderly women involved in a weekly exercise program: A randomized controlled trial. American Journal of Clinical Nutrition, 100(3), 974-981.

Vest, A. R., Chan, M., Deswal, A., Givertz, M. M., Lekavich, C., Lennie, T., Litwin, S. E., Parsly, L., Rodgers, J. E., ... Desai, A. (2019). Nutrition, Obesity, and Cachexia in Patients With Heart Failure: A Consensus Statement from the Heart Failure Society of America Scientific Statements Committee. Journal of Cardiac Failure, 25(5), 380-400.

Viethen, T., Gerhardt, F., Dumitrescu, D., Knoop-Busch, S., Ten Freyhaus, H., Rudolph, T. K., Baldus, S., \& Rosenkranz, S. (2014). Ferric carboxymaltose improves exercise capacity and quality of life in patients with pulmonary arterial hypertension and iron deficiency: A pilot study. International Journal of Cardiology, 175(2), 233-239.

Vinke, P., Jansen, S. M., Witkamp, R. F., \& van Norren, K. (2018). Increasing quality of life in pulmonary arterial hypertension: is there a role for nutrition? Heart Failure Reviews, 23(5), 711-722.

Voelkel, N. F., Gomez-Arroyo, J., Abbate, A., Bogaard, H. J., \& Nicolls, M. R. (2014). Pathobiology of pulmonary arterial hypertension and right ventricular failure. European Respiratory Journal, 40(6), 1555-1565.

von Haehling, S., Anker, M. S., \& Anker, S. D. (2016). Prevalence and clinical impact of cachexia in chronic illness in Europe, USA, and Japan: facts and numbers update 2016. Journal of Cachexia, Sarcopenia and Muscle, 7(5), 507-509. 
von Haehling, S., Doehner, W., \& Anker, S. D. (2007). Nutrition, metabolism, and the complex pathophysiology of cachexia in chronic heart failure. Cardiovascular Research, 73(2), 298-309.

von Haehling, S., Ebner, N., dos Santos, M. R., Springer, J., \& Anker, S. D. (2017). Muscle wasting and cachexia in heart failure: mechanisms and therapies. Nature Reviews Cardiology, 14(6), 323-341.

von Haehling, S., Lainscak, M., Springer, J., \& Anker, S. D. (2009). Cardiac cachexia: A systematic overview. Pharmacology and Therapeutics, 121(3), 227-252.

Waldron, J. L., Ashby, H. L., Cornes, M. P., Bechervaise, J., Razavi, C., Thomas, O. L., Chugh, S., Deshpande, S., Ford, C., \& Gama, R. (2013). Vitamin D: A negative acute phase reactant. Journal of Clinical Pathology, 66(7), 620-622.

Weinstein, A. A., Chin, L. M. K., Keyser, R. E., Kennedy, M., Nathan, S. D., Woolstenhulme, J. G., Connors, G., \& Chan, L. (2013). Effect of aerobic exercise training on fatigue and physical activity in patients with pulmonary arterial hypertension. Respiratory Medicine, 107(5), 778-784.

Welch, A. A., Kelaiditi, E., Jennings, A., Steves, C. J., Spector, T. D., \& MacGregor, A. (2016). Dietary Magnesium Is Positively Associated with Skeletal Muscle Power and Indices of Muscle Mass and May Attenuate the Association between Circulating C-Reactive Protein and Muscle Mass in Women. Journal of Bone and Mineral Research, 31(2), 317-325.

Wilhelm, S. M., Rjater, R. G., \& Kale-Pradhan, P. B. (2013). Perils and pitfalls of longterm effects of proton pump inhibitors. Expert Review of Clinical Pharmacology, 6(4), 443-451.

William, J. H., \& Danziger, J. (2016). Proton-pump inhibitor-induced hypomagnesemia: Current research and proposed mechanisms. World Journal of Nephrology, 5(2), 152-157.

Winther, G., Pyndt Jørgensen, B. M., Elfving, B., Nielsen, D. S., Kihl, P., Lund, S., Sorensen, D. B., \& Wegener, G. (2015). Dietary magnesium deficiency alters gut microbiota and leads to depressive-like behaviour. Acta Neuropsychiatrica, 27(3), 168-176.

Witham, W. G., Yester, K. A., \& McGaffin, K. R. (2013). A high leucine diet mitigates cardiac injury and improves survival after acute myocardial infarction. Metabolism: Clinical and Experimental, 62(2), 290-302. 
Witkamp, R. F., \& van Norren, K. (2018). Let thy food be thy medicine....when possible. European Journal of Pharmacology, 836, 102-114.

Witkowski, M., Hubert, J., \& Mazur, A. (2011). Methods of assessment of magnesium status in humans: A systematic review. Magnesium Research, 24(4), 163-180.

Wortsman, J., Matsuoka, L. Y., Chen, T. C., Lu, Z., \& Holick, M. F. (2000). Decreased bioavailability of vitamin D in obesity. American Journal of Clinical Nutrition, 72(3), 690-693.

Wynn, T. A., \& Ramalingam, T. R. (2012). Mechanisms of fibrosis: therapeutic translation for fibrotic disease. Nature Medicine, 18(7), 1028-1040.

Yang, J. mei, Zhou, R., Zhang, M., Tan, H. ran, \& Yu, J. qiang. (2018). Betaine attenuates monocrotaline-induced pulmonary arterial hypertension in rats via inhibiting inflammatory response. Molecules, 23(6), 1-15.

Young, G., \& Jewell, D. (2002). Interventions for leg cramps in pregnancy. Cochrane Database of Systematic Reviews, 1, CD000121.

Yu, L. Y., Sun, L. N., Zhang, X. H., Li, Y. Q., Yu, L., Yuan, Z. Q. Y., Meng, L., Zhang, H. W., \& Wang, Y. Q. (2017). A Review of the Novel Application and Potential Adverse Effects of Proton Pump Inhibitors. Advances in Therapy, 34(5), 10701086.

Zafrir, B. (2013). Exercise Training and Rehabilitation in Pulmonary Arterial Hypertension. Journal of Cardiopulmonary Rehabilitation and Prevention, 33(5), 263-273.

Zamanian, R. T., Hansmann, G., Snook, S., Lilienfeld, D., Rappaport, M., Reaven, G. M., Rabinovitch, M., \& Doyle, R. L. (2010). Insulin Resistance in Pulmonary Arterial Hypertension. Eur Respir J., 33(2), 318-324.

Zhang, X., Zhou, S., Pan, K., Li, X., Zhao, X., Zhou, Y., Cui, Y., \& Liu, X. M. (2017). Potentially inappropriate medications in hospitalized older patients: A crosssectional study using the Beers 2015 criteria versus the 2012 criteria. Clinical Interventions in Aging, 12, 1697-1703.

Zhou, S., Li, M. T., Jia, Y. Y., Liu, J. J., Wang, Q., Tian, Z., Liu, Y. T., Chen, H. Z., Liu, D. P., \& Zeng, X. F. (2015). Regulation of cell cycle regulators by SIRT1 contributes to resveratrol-mediated prevention of pulmonary arterial hypertension. BioMed Research International, 2015, 762349. 
Zittermann, A. (2003). Vitamin D in preventive medicine: are we ignoring the evidence? British Journal of Nutrition, 89(5), 552-572. 


\section{Appendix I}

Additional information for chapter 3

$\mathrm{Hb}$

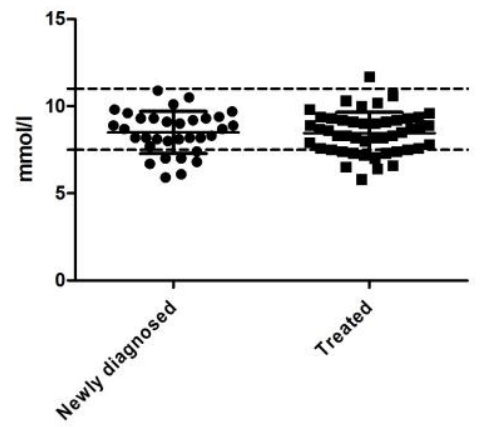

Ferritin

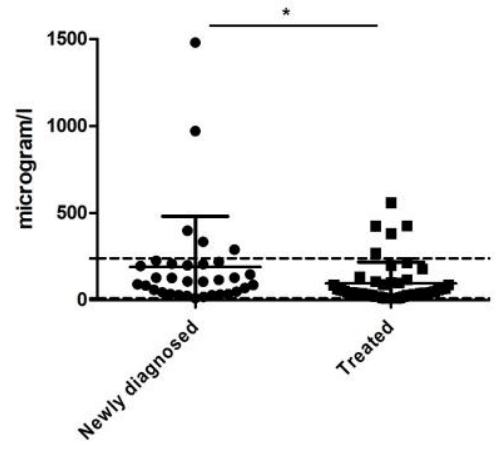

Iron

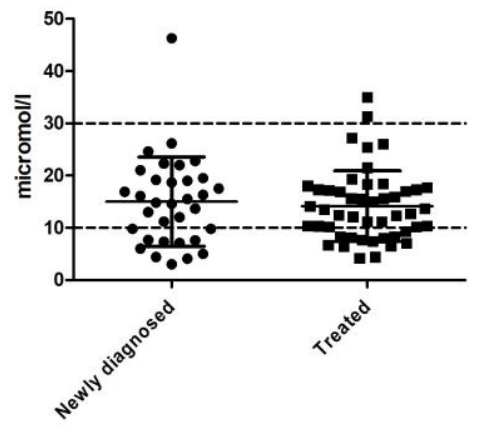

Sat. transferrin

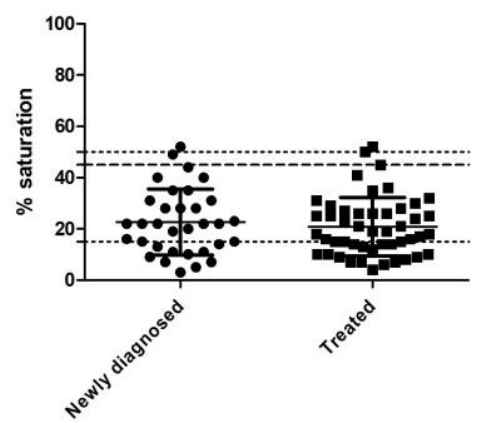

Figure A.I.1: Levels of $\mathrm{Hb}$, iron and ferritin and transferrin saturation of newly diagnosed and treated patients (PAH and CTEPH mixed). 
$\mathrm{Hb}$ male

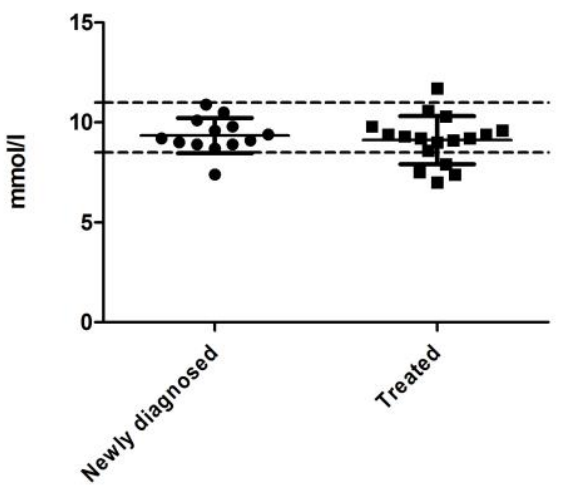

$\mathrm{Hb}$ female

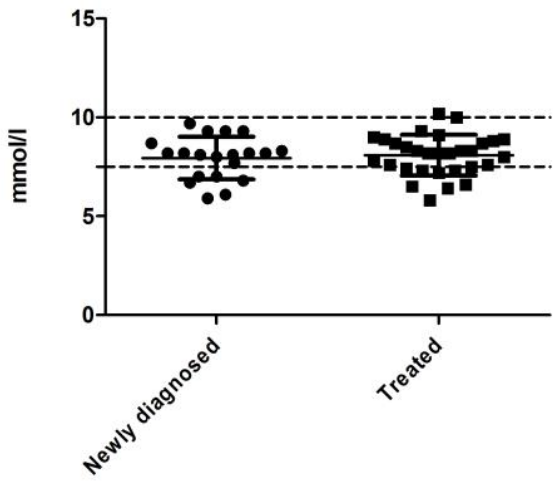

Figure A.I.2: Hemoglobin levels of newly diagnosed and treated male and female patients (PAH and CTEPH mixed).

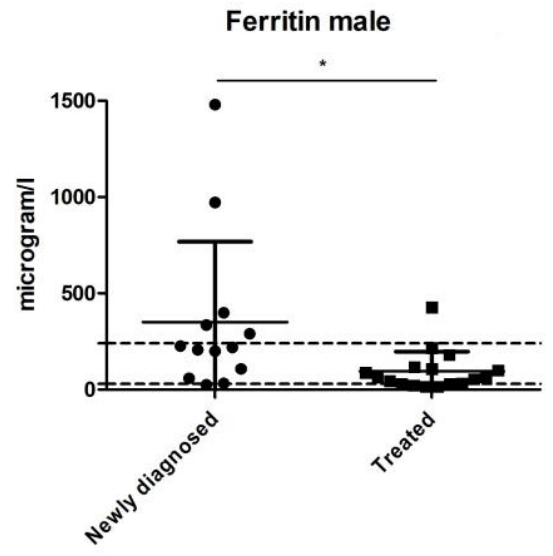

Ferritin female

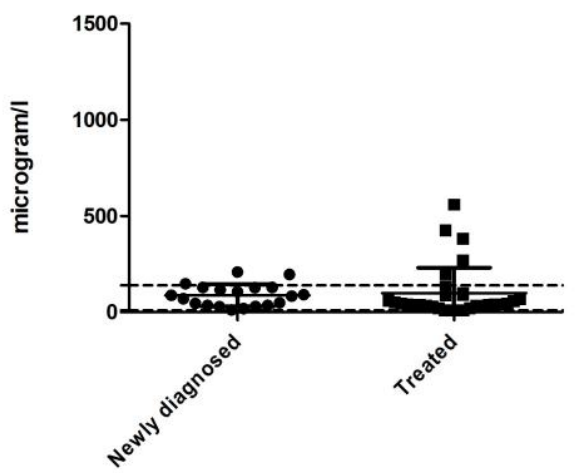

Figure A.I.3: Ferritin levels of newly diagnosed and treated male and female patients (PAH and CTEPH mixed). 

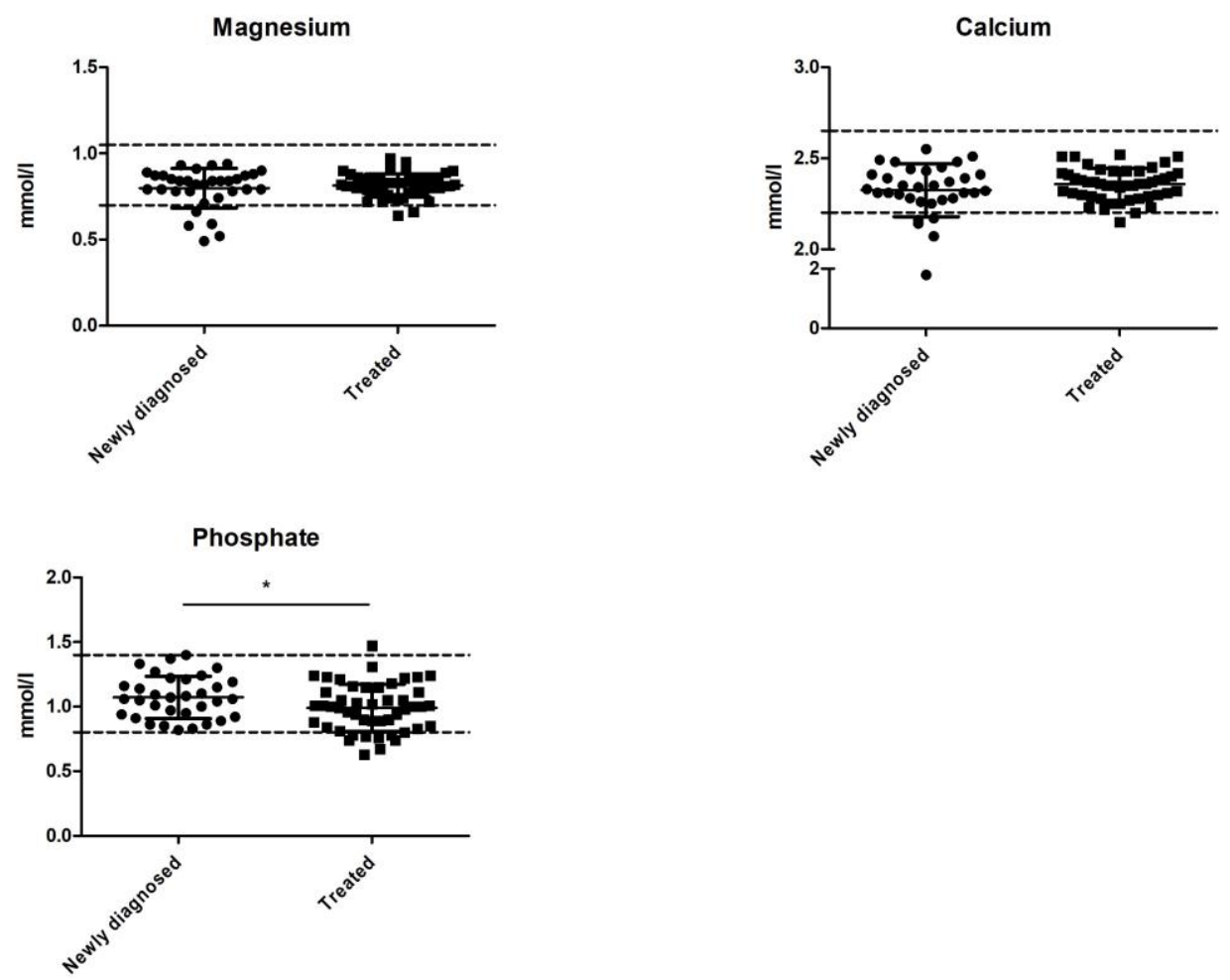

Figure A.I.4 Levels of magnesium, calcium and phosphate in newly diagnosed and treated patients (PAH and CTEPH mixed). 

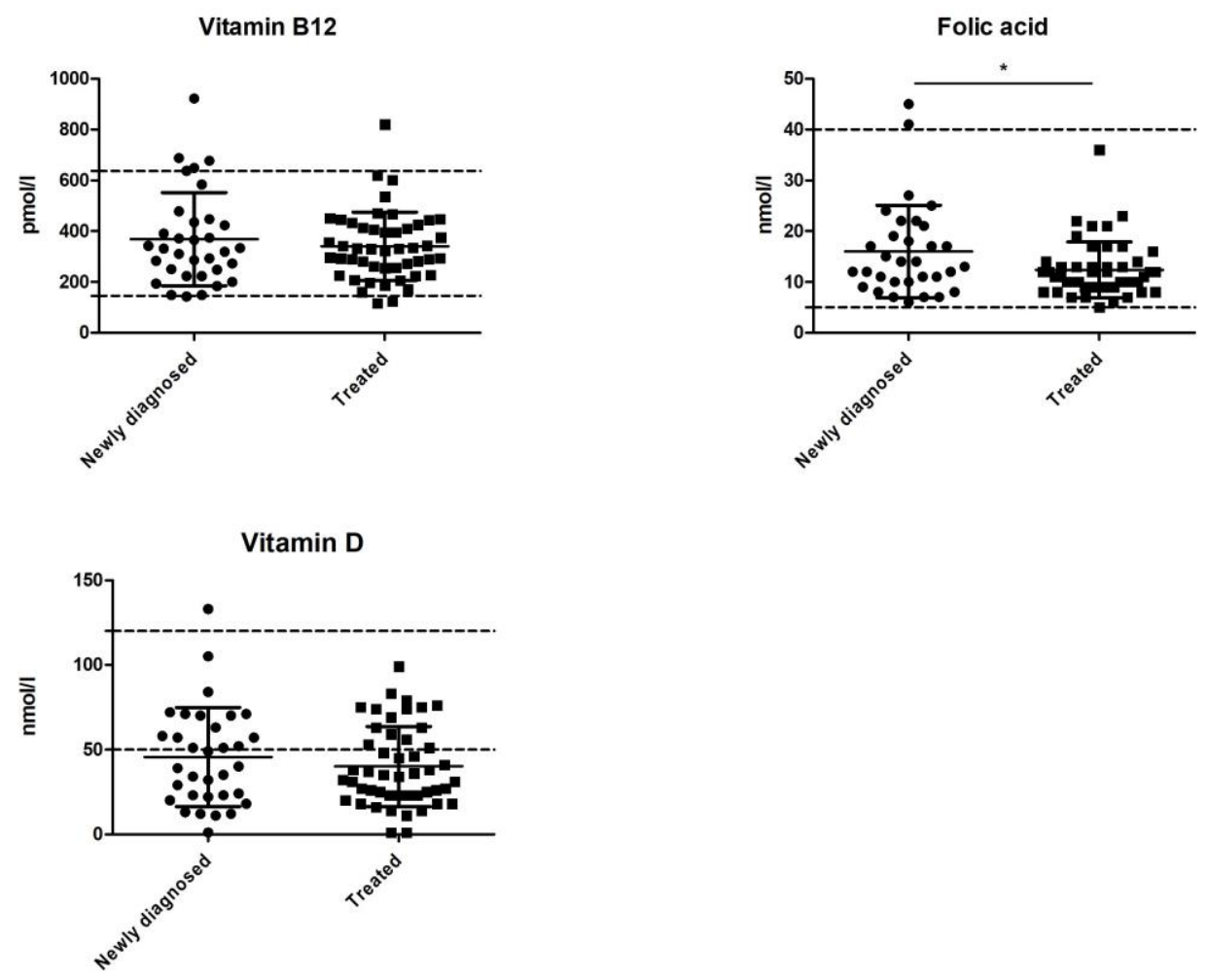

Figure A.I.5: Levels of vitamin B12, folic acid and vitamin D in newly diagnosed and treated patients (PAH and CTEPH mixed). 


\section{Appendix II}

Additional information for chapter 3
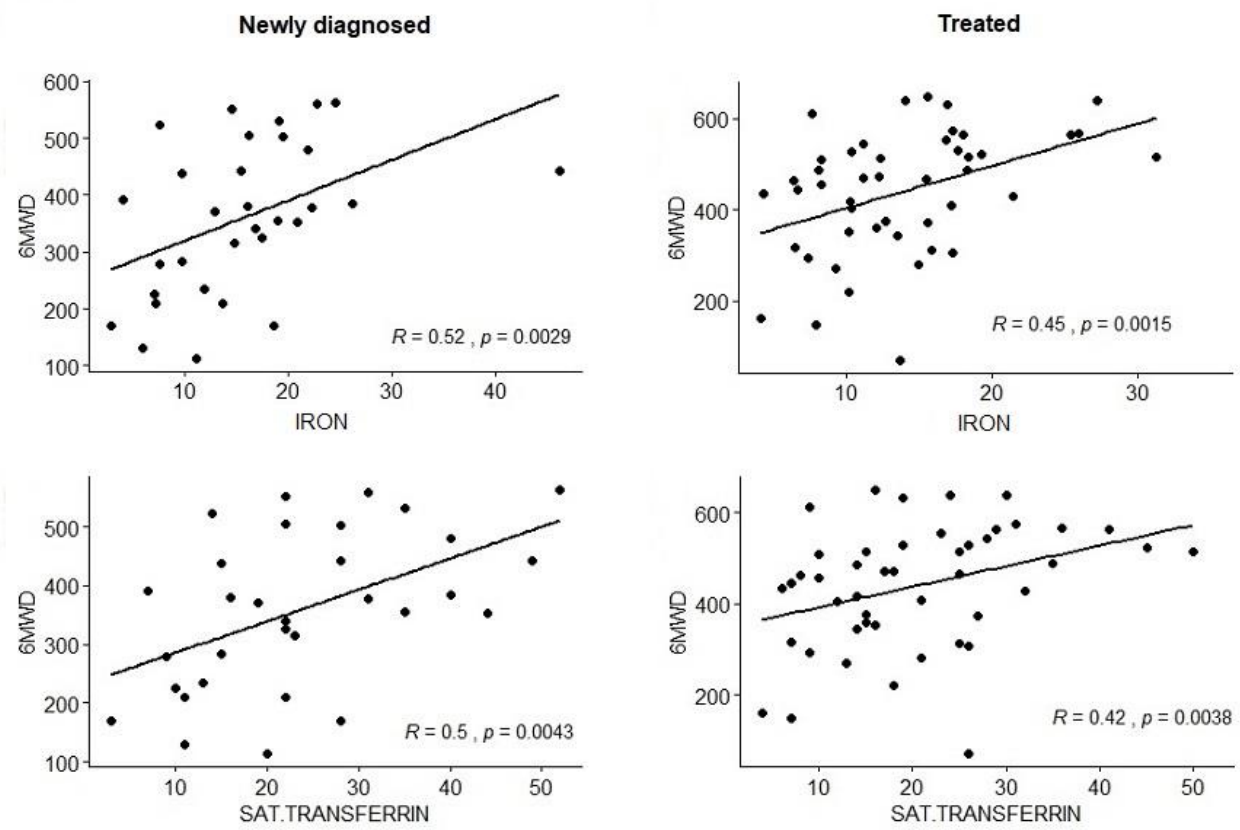

Figure A.II.1: Correlation 6MWD with iron and transferrin saturation levels in PAH and CTEPH patients. 
Treated

NT-Pro-BNP

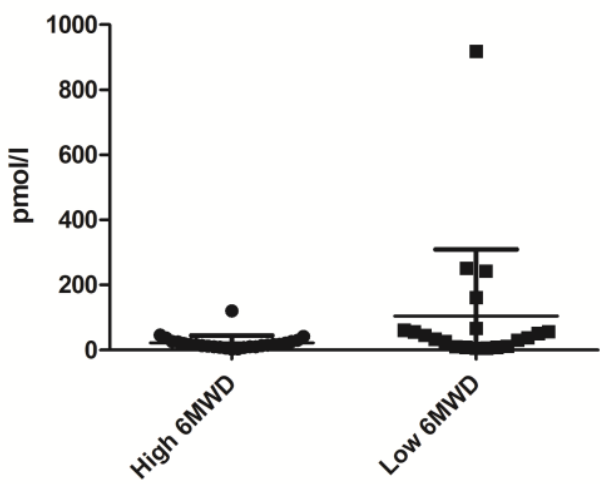

Newly diagnosed

NT-Pro-BNP

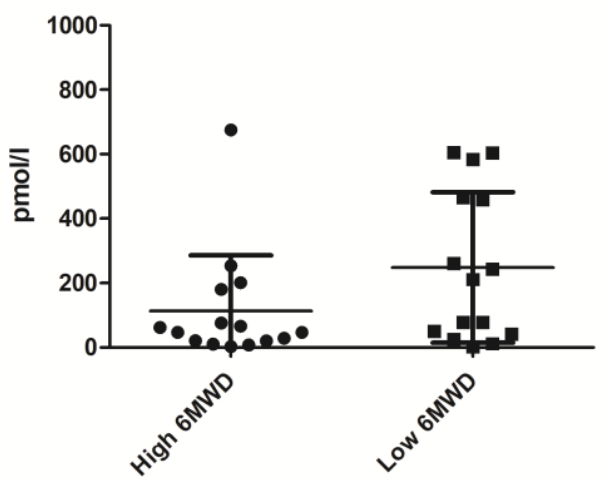

Figure A.II.2: NT-Pro-BNP levels of newly diagnosed and treated patients (PAH and CTEPH mixed) with 6MWD below and above the mean. 


\section{Appendix III}

Additional information for chapter 5

\section{Food intake}

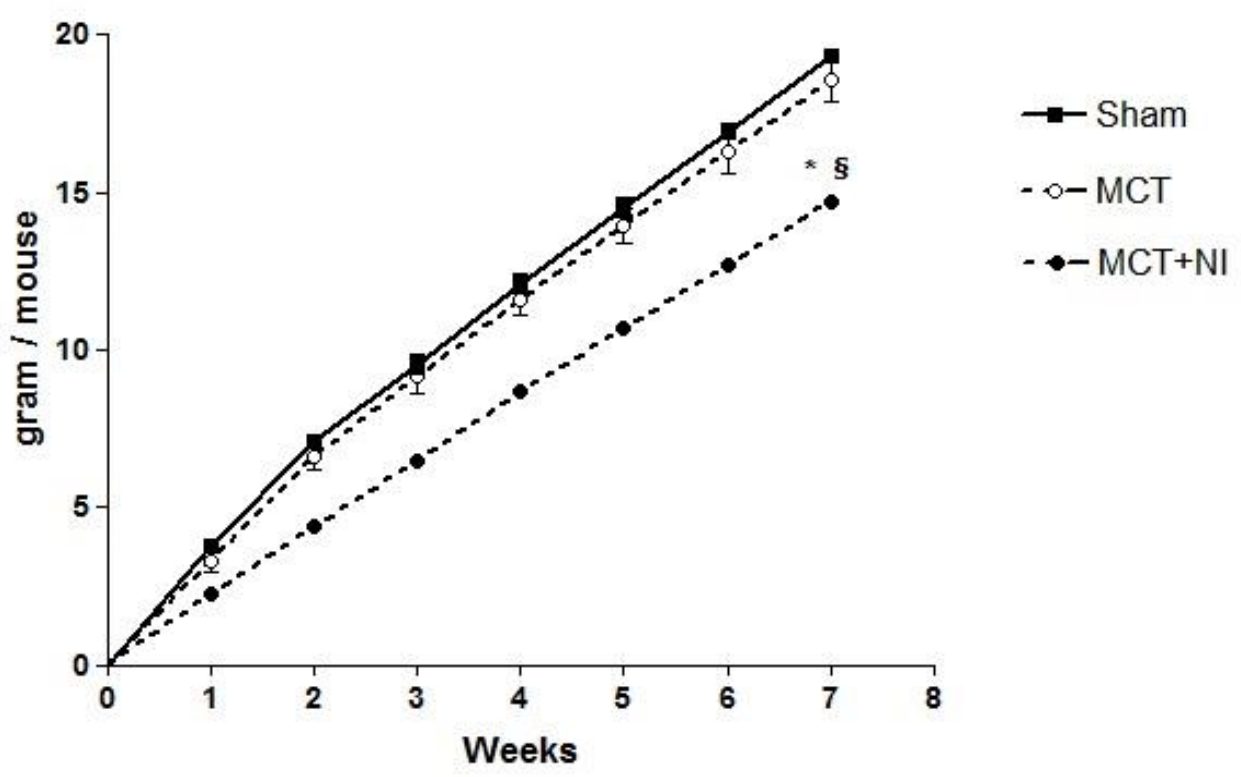

Figure A.III: Cumulative food intake after 7 weeks was similar in sham and MCT mice, but lower in MCT+NI mice compared to MCT and sham mice. $\left(^{*} \mathrm{p}<0.01\right.$ versus sham; $\S \mathrm{p}<0.05$ versus $\mathrm{MCT}$ ) 


\section{Appendix IV}

Additional information for chapter 5
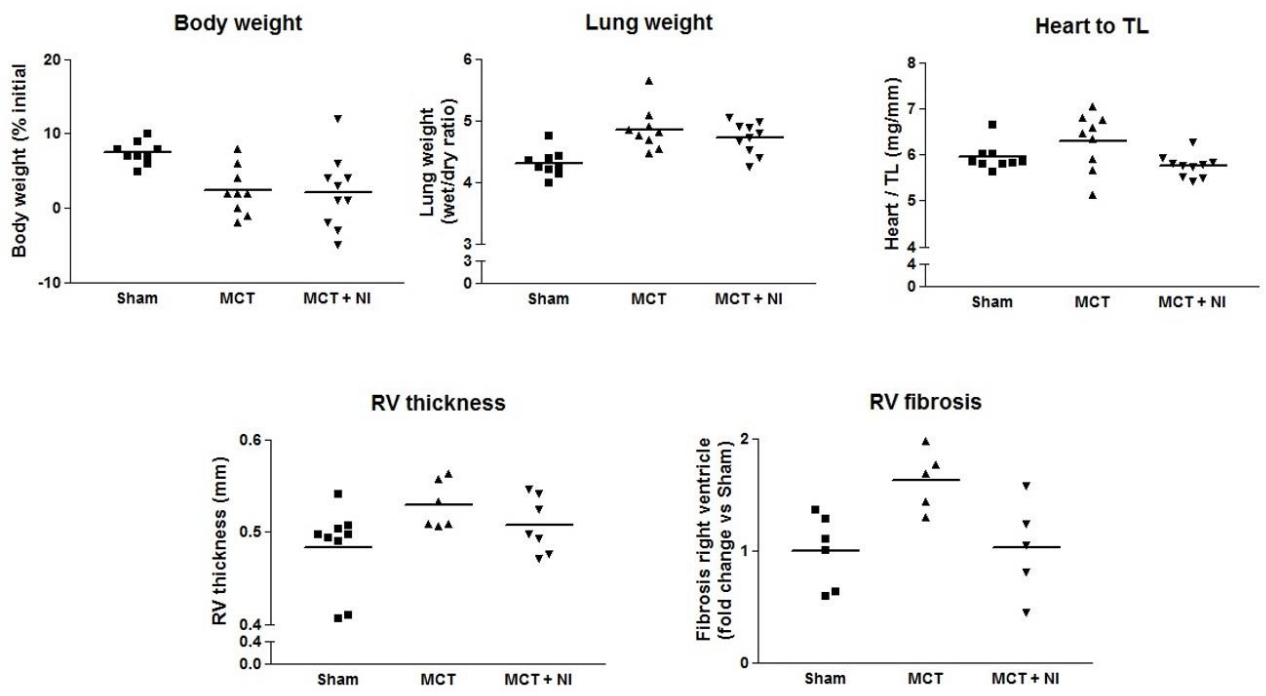

Figure A.IV: Scatterplots showing the variation of the physiological data depicted in figures 1 and 2 of the main manuscript. 


\section{Appendix V}

Additional information for chapter 5

Supplementary datasets 1-4 are published separately with the article and can be found at: https://doi.org/10.1038/s41598-019-46331-4.

Supplementary dataset 1: GSEA Nutrition PAH RV MCT_vs_sham negative.xls Supplementary dataset 2: GSEA Nutrition PAH RV MCT_vs_sham positive.xls Supplementary dataset 3: GSEA Nutrition PAH RV MCT+NI_vs_MCT negative.xls Supplementary dataset 4: GSEA Nutrition PAH RV MCT+NI_vs_MCT positive.xls

Reference:

Vinke, Paulien, T. Scott Bowen, Mark V. Boekschoten, Renger F. Witkamp, Volker Adams, and Klaske van Norren. 2019. 'Anti-Inflammatory Nutrition with High Protein Attenuates Cardiac and Skeletal Muscle Alterations in a Pulmonary Arterial Hypertension Model'. Scientific Reports 9 (1): 10160. 


\section{Appendix VI}

Additional information for chapter 5

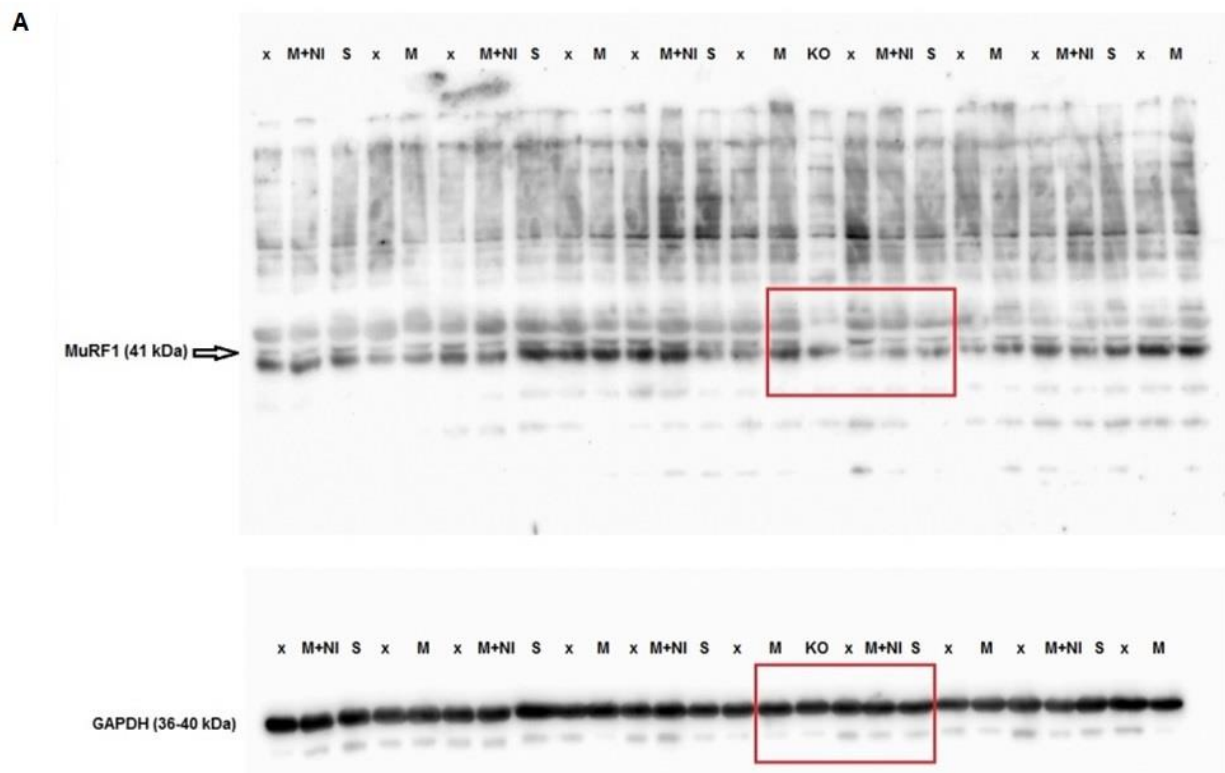

Figure A.VIA: Complete blot of MuRF1 and GAPDH on the same blot shown in figure 7A. The red boxes depict the area used in the cropped figure 7A. A MuRF1 knock-out (KO) sample was included to separate the MuRF1 bands from other bands due to nonspecific binding of the antibody. GAPDH was measured after MuRF1 incubation. 
B

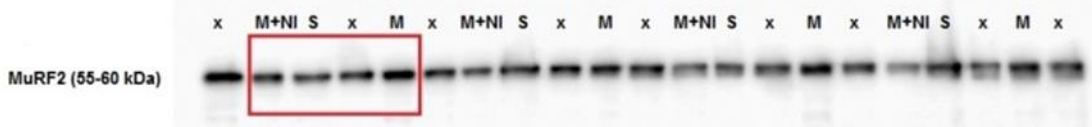

GAPDH (36-40 kDa)

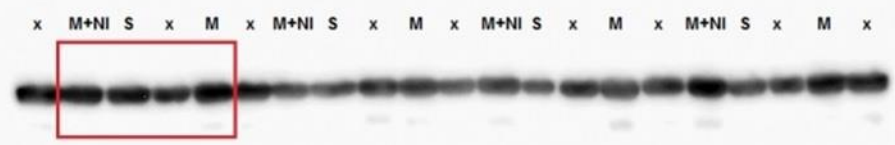

Figure A.VIB: Complete blot of MuRF2 and GAPDH on the same blot shown in figure 7B. The red boxes depict the area used in the cropped figures in figure 7B. GAPDH was measured after MuRF2 incubation 


\section{English summary}

Pulmonary arterial hypertension (PAH) is a chronic and progressive disease characterized by vasoconstriction and structural remodelling of the pulmonary arteries. Increased vascular resistance and elevated pulmonary arterial pressures contribute to right ventricular hypertrophy and eventually lead to heart failure and premature death. Symptoms of PAH include serious exercise intolerance, reducing the quality of life of patients. Nutrition is increasingly being recognized as an important factor contributing to quality of life during disease, especially when exercise intolerance is present. There is however limited knowledge on the role of nutrition in PAH and nutritional guidelines are scarce. In the meantime, PAH patients are actively seeking advice on such matters. In the clinic, assessment of nutritional status or micronutrient concentrations are currently not standard practice and nutritional intervention is not part of regular treatment, due to the lack of evidence-based recommendations for PAH patients.

In this thesis, the role of nutrition in $\mathrm{PAH}$ pathophysiology and the potential of nutritional intervention in the treatment of $\mathrm{PAH}$ is being explored. The aim of this thesis is to gain more knowledge on the prevalence of micronutrient deficiencies of patients with $\mathrm{PAH}$ and to explore possible nutritional interventions to reduce fatigue and improve exercise tolerance and quality of life in PAH patients. These objectives are addressed by 1) literature research and hypothesis generation, 2) measurement of micronutrient status in PAH and CTEPH patients, 3) exploration of the relation between levels of micronutrients and clinical and patient-related outcomes and 4) a study on the effect of nutritional intervention on muscle function in a mouse model of PAH.

Chapter 2 summarizes current insights in the pathophysiological mechanisms underlying PAH. It zooms in on the potential involvement of nutritional status and micronutrient deficiencies on $\mathrm{PAH}$ exercise intolerance and fatigue, also summarizing the potential benefits of exercise and nutritional interventions. Pathophysiological processes that contribute to exercise intolerance and impaired quality of life in $\mathrm{PAH}$ patients include right ventricular dysfunction, inflammation, 
skeletal muscle alterations and dysfunctional energy metabolism. Moreover, $\mathrm{PH}-$ related nutritional deficiencies and metabolic alterations are linked to fatigue, exercise intolerance and endothelial dysfunction. Available evidence suggests that exercise interventions can be effective in $\mathrm{PAH}$ patients to improve exercise tolerance and decrease fatigue. By contrast, knowledge on the prevalence of micronutrient deficiencies and the possible effects of nutritional interventions in PAH patients is limited. Although data on nutritional status and micronutrient deficiencies in $\mathrm{PAH}$ are scarce, the available knowledge, including that from adjacent fields, suggests that nutritional intervention to correct deficiencies and metabolic alterations may contribute to a reduction of disease burden.

In Chapter 3, the prevalence of micronutrient deficiencies in both newly diagnosed and treated patients with $\mathrm{PAH}$ and chronic thrombo-embolic pulmonary hypertension (CTEPH) are described and correlations between micronutrients and clinical outcomes are assessed.

Deficiencies in both iron and vitamin D are highly prevalent in both PAH and CTEPH patients at diagnosis as well as after 1,5 years of treatment. In the newly diagnosed group, $42 \%$ of $\mathrm{PAH}$ patients and $21 \%$ of CTEPH patients were iron deficient, compared to $29 \%$ of PAH patients and $20 \%$ of CTEPH patients in the treatment group. Vitamin D deficiency occurred in $42 \%$ of the newly diagnosed PAH patients, in $71 \%$ of the newly diagnosed CTEPH patients, in $68 \%$ of the treated PAH patients and in $70 \%$ of the treated CTEPH patients.

Iron plasma levels correlate with 6-minute walk distance. Low iron levels seem to be related to inflammation via hepcidin levels. Vitamin D levels did not correlate with any of the outcome measures in our study. Therefore, the relevance of vitamin $\mathrm{D}$ deficiency in PAH and CTEPH patients remains unclear and further studies into this topic are needed. The high prevalence of deficiencies in vitamin D and iron however underline the need for monitoring their status. Studies evaluating the possible effects and safety of different supplementation strategies for iron as well as vitamin D in PAH and CTEPH patients are necessary.

Chapter 4 discusses the hypothesis that the use of proton pump inhibitors (which are highly used by PAH patients) can contribute to a reduction in muscle mass and function in chronically ill patients. Literature indicates that a PPI-induced reduction 
in acidity of the gastrointestinal tract can decrease the absorption of, amongst others, magnesium. Low levels of magnesium are associated with impaired muscle function. This unwanted side-effect of PPIs on muscle function has been described in different disease backgrounds, but has not been studied in PAH. Magnesium is necessary for activation of vitamin D. Low vitamin $D$ and magnesium levels together can lead to increased inflammation involved in muscle wasting. In addition, PPI use has been described to alter the microbiota's composition in the gut, which might lead to increased inflammation. However, PPIs are often provided together with nonsteroidal anti-inflammatory drugs (NSAIDs). In conclusion, use of PPIs has been reported to contribute to muscle function loss. Whether this will add to the risk factor for development of muscle function loss in patients with PAH needs further investigation.

Chapter 5 describes the effect of a multi-targeted nutritional intervention with extra protein, leucine, and the anti-inflammatory components of fish oil and oligosaccharides on pathological changes in cardiac and skeletal muscle of a PAH mouse model. PAH was induced in female C57/BL6 mice by weekly injection of monocrotaline (MCT) for 8 weeks. One MCT group and the sham group received a control diet, while another MCT group received the isocaloric nutritional intervention. The results show that, compared to sham, MCT mice have an increase in heart weight, RV thickness and fibrosis, which were attenuated in MCT+NI mice. Microarray and qRT-PCR analysis on the RV confirmed effects on fibrotic pathways. Skeletal muscle fiber cross-sectional area (CSA) of the TA was reduced in MCT compared to sham mice, but preserved in the MCT+NI group. In the EDL a similar effect was seen. The findings show that nutritional intervention significantly attenuated changes in both cardiac and skeletal muscle in a mouse model of PAH, providing directions for future therapeutic strategies targeting functional decline of both tissues.

The combined results of this thesis show that inflammation is a recurring factor in the pathophysiology of PAH that is not only related to disease outcome, but also to nutritional factors. Recommendations for the clinic and future directions for research are provided in chapter 6 . The future has to reveal the full potential of nutritional intervention to complement treatment strategies in PAH. 


\section{Nederlandse samenvatting}

Pulmonale arteriële hypertensie (PAH) is een chronische, progressieve ziekte gekarakteriseerd door vasoconstrictie en structurele veranderingen in de longaderen. Verhoogde veneuze druk en verhoogde bloeddruk in de longaderen leiden tot vergroting van de rechter hartkamer en uiteindelijk tot hartfalen en een vervroegde dood. Een belangrijk symptoom van $\mathrm{PAH}$ is ernstige bewegingsintolerantie wat de kwaliteit van leven van PAH-patiënten verlaagd. Voeding wordt toenemend gezien als een belangrijke factor die bijdraagt aan levenskwaliteit tijdens chronische ziekte, vooral als er sprake is van bewegingsintolerantie. Helaas is er maar beperkte informatie beschikbaar over de rol van voeding in PAH en voedingsrichtlijnen zijn schaars. In de tussentijd zoeken PAH-patiënten toenemend informatie en advies rondom voeding. In de klinische setting wordt bepaling van de voedingsstatus of deficiënties in micronutriënten (vitamines en mineralen) niet standaard uitgevoerd. Voedingsinterventies zijn geen onderdeel van de reguliere zorg, vanwege het gebrek aan wetenschappelijk onderbouwde aanbevelingen voor PAH-patiënten

In dit proefschrift wordt de rol van voeding in de pathofysiologie van PAH bestudeerd en het potentiaal van voedingsinterventie in de behandeling van $\mathrm{PAH}$ onderzocht. Het doel van dit proefschrift is om meer kennis te vergaren rondom de prevalentie van deficiënties in micronutriënten in PAH-patiënten. Ook worden mogelijke voedingsinterventies onderzocht om vermoeidheid en bewegingsintolerantie te verminderen en de kwaliteit van leven van PAH-patiënten te verbeteren. Deze doelen worden bereikt door middel van 1) literatuuronderzoek en hypothesegeneratie, 2) meting van de micronutriëntstatus in PAH- en CTEPH atiënten, 3) onderzoek naar de relatie tussen micronutriënten en klinische en patiëntgerelateerde uitkomstmaten en 4) een studie naar het effect van voedingsinterventie op de spierfunctie in een muismodel van PAH.

Hoofdstuk 2 vat de huidige inzichten omtrent de pathofysiologische mechanismen in PAH samen. Het zoomt in op de rol van de voedingsstatus en deficiënties aan micronutriënten op de bewegingsintolerantie en vermoeidheid van PAH-patiënten. 
Bovendien vat het de mogelijke voordelen van bewegings- en voedingsinterventies samen. Ziekteprocessen die bijdragen aan vermoeidheid en bewegingsintolerantie zijn een dysfunctionele rechter hartkamer, onstekingsprocessen, veranderingen in de skeletspieren en in het energiemetabolisme. Typische voedingsdeficiënties in $\mathrm{PAH}$ zijn bovendien gerelateerd aan veranderingen in het endotheel van de vaten. Er is wetenschappelijk bewijs dat therapie in de vorm van sport en bewegen effectief kan zijn om bewegingsintolerantie en vermoeidheid in PAH-patiënten tegen te gaan. Wetenschappelijk bewijs over het effect van voedingsinterventies in PAH is helaas beperkt. De beschikbare informatie, in combinatie met wetenschappelijke kennis uit gerelateerde vakgebieden, laat wel zien dat voedingsinterventies om deficiënties en metabole veranderingen te corrigeren kunnen bijdragen aan een verminderde ziektelast.

In hoofdstuk 3 wordt de prevalentie van micronutriënten deficiënties in net gediagnosticeerde en behandelde PAH- en CTEPH-patiënten beschreven en correlaties tussen micronutriënten en klinische uitkomstmaten besproken. Deficiënties in ijzer en vitamine D komen veel voor in zowel PAH als CTEPHpatiënten en zowel bij diagnose als na 1,5 jaar behandeling. In de net gediagnosticeerde groep heeft $42 \%$ van de PAH en $21 \%$ van de CTEPH-patiënten ijzer deficiëntie, vergeleken met $29 \%$ van de PAH-patiënten en $20 \%$ van de CTEPHpatiënten in de behandelde groep. Deficiëntie in vitamine D kwam in $42 \%$ van de net gediagnosticeerde PAH-patiënten, in 71\% van de net gediagnosticeerde CTEPHpatiënten, in $68 \%$ van de behandelde $\mathrm{PAH}$-patiënten en in $70 \%$ van de behandelde CTEPH-patiënten voor. Plasma niveaus van ijzer correleerden met de 6 minuten looptest. Lage ijzerwaarden leken gerelateerd aan ontstekingsprocessen via hepcidine waarden. Vitamine D waarden correleerden met geen enkele uitkomstmaat in deze studie. Daarom is de relevantie van vitamine $\mathrm{D}$ deficiënties in PAH- en CTEPH-patiënten onzeker. Verdere studies naar dit onderwerp zijn nodig. De hoge prevalentie aan ijzer en vitamine $\mathrm{D}$ deficiëntie maakt wel duidelijk dat er een noodzaak bestaat om deze waarden regelmatig te monitoren. Studies om het effect en de veiligheid van supplementatie van ijzer en vitamine D in PAH en CTEPH-patiënten zijn nodig. 
Hoofdstuk 4 behandelt de hypothese dat het gebruik van proton pomp inhibitoren (PPIs) (die veelvuldig worden gebruikt door PAH-patiënten) kunnen bijdragen aan een vermindering van de massa en functie van de spieren in chronisch zieken. PPIs leiden tot een vermindering van de zuurtegraad van het maagdarmstelsel, waardoor de absorptie van o.a. magnesium verminderd. Lage magnesium waarden zijn geassocieerd met verminderde spierfunctie. Magnesium is nodig voor de activatie van vitamine $D$. Lage vitamine $D$ en magnesium waarden kunnen leiden tot verhoogde ontstekingsprocessen, wat weer kan leiden tot verlies van spiermassa. Bovendien is beschreven dat PPI gebruik de samenstelling van de darm microbiota kan veranderen, wat ook weer tot verhoogde ontstekingsprocessen kan leiden. PPIs worden echter vaak samen met ontstekingsremmers (NSAIDs) gegeven. Eventuele ongewilde neveneffecten van PPI gebruik op de spieren in PAH-patiënten moet verder bestudeerd worden.

Hoofdstuk 5 beschrijft het effect van een multi-target voedingsinterventie met extra eiwit, leucine en de ontstekingsremmende componenten visolie en oligosacchariden op pathologische veranderingen in het hart en de skeletspieren in een $\mathrm{PAH}$ muismodel. PAH was geïntroduceerd in vrouwelijke C57/BL6 muizen door wekelijkse injectie met monocrotaline (MCT) gedurende 8 weken. Een MCT groep en de controle (sham) groep ontvingen een controlevoeding, terwijl een andere MCT groep de isocalorische voedingsinterventie kreeg. De resultaten laten zien dat MCT muizen een vergroot hart, een verdikte rechter hartkamer en fibrose ontwikkelden en dat deze symptomen in MCT muizen die de voedingsinterventie kregen zich minder ontwikkelden. Microarray en qRT-PCR analyse op de rechter hartkamer bevestigden het omhoog reguleren van fibrotische signaalwegen. De dikte van spiervezels van de tibialis anterior en extensor digitorum longes was verminderd in MCT muizen, maar behouden in de MCT muizen die de voedingsinterventie kregen. De bevindingen laten zien dat voedingsinterventie belangrijke pathofysiologische veranderingen in een muismodel van PAH verminderden. Dit geeft een potentieel aan voor voeding als toekomstige behandelingsmogelijkheid om functionele vermindering van hart en spieren in PAH tegen te gaan.

De resultaten van dit proefschrift laten zien dat onstekingsprocessen een terugkerende factor zijn in de pathofysiologie van PAH die niet alleen gerelateerd 
zijn aan de prognose van de patiënt, maar ook aan voedingsfactoren. Klinische aanbevelingen en suggesties voor vervolgonderzoek staan beschreven in hoofdstuk 6. De toekomst zal het volledige potentiaal van voedingsinterventies om bestaande behandelingsstrategieën voor PAH te complementeren nog moeten uitwijzen. 


\section{Acknowlegdements}

During the last 6 years, there were many moments where it seemed almost impossible that this PhD thesis would ever be completed. Now, it seems a miracle that it is finished. There are many people who made the journey possible and I would like to thank all of you.

My supervisors Klaske van Norren and Renger Witkamp for supporting my idea to write grants and start my own $\mathrm{PhD}$ project in an unconventional manner. Klaske, I am incredibly grateful for having you as my daily supervisor. You have been kind, motivating, critical, funny, caring and supporting during this whole adventure. It has been a difficult one, but I always felt that I could count on you and discuss anything possible. I learned a lot from your style of teaching, networking and doing research. I look back at many nice make-your-own-pizza dinners at your home during my stays in the Netherlands. In Germany, supervisors of doctoral students are traditionally called 'doktorvater'. You have been what one could call a 'doctormother' to me. Without your support I would not have been able to start or finish this PhD.

Renger, you also have been incredibly supporting and motivating during the whole process. Your energy and writing skills are an example to me and I hope to have taken something from them. You and Klaske were also always interested in my adventures with I am a Foodie outside the university, which I appreciate a lot.

Mieke, thank you for being such a great colleague, for the fun we had during (preparations for) teaching practicals and coffee breaks or lunches and for your help with the laboratory work when I was with pregnancy leave. And afterwards, during covid-19 times, for the chats about our Nora(h)s and lockdown life. Michiel, thank you for many good talks we had over the years, for your support and enthusiasm for my PhD project and for being a critical and always helpful co-author. Mark and Guido, thank you for your support with the microarray analysis for my animal study. Jocelijn, thank you for the possibility of becoming more teaching experience in your MSc course and for the good talks we had during my stays in Wageningen. Vera and Wout: thank you for working on the hypothesis paper of this thesis 
together. I am very happy about the fast and constructive way in which we were able to write it. And of course also about the times in Wageningen where we met during coffees, parties and teaching obligations.

All of my other colleagues at Wageningen University: Jvalini, Nikkie, Inge, Carolien, Miranda, Rogier, Xiaolin, Charlotte, Xanthe, Mara, Merel, Pol, Harm, Tessa, Sophie, Benthe, Anouk, Montse, Roland, Philip, Francesca, Rieneke, Ian, Suzanne, Susanne and many others. Thank you for the great times we had during breaks, while being active at the WUR days, and during parties and conferences.

Volker, without your help this thesis would not have existed. Thank you for giving me the chance to work in your lab in Leipzig, for all the support and materials to be able to perform the animal study and for being a kind and critical supervisor during the process. I felt very much at home in your team, where I have learned so much and I am very grateful for that.

Scott, thank you for being such a good mentor to me during our time at the Leipzig Heart Center. Your critical view and hard work have inspired me. You taught me how to network at conferences, how to write critically and keep improving yourself.

My other colleagues at the University of Leipzig, Heart Center: Petra, Tina, Sarah, Angela, Ilka, Basti, Maya, Kristin, Zelia, Christian, Lena, Dominic, Sophia and Justus. Thank you for many great years in Leipzig, for the good times during long days in the lab, the birthday breakfasts, lunches in the sun, the dancing (in the lab and beyond) and the beers outside of work.

My collaborators at the Erasmus MC Rotterdam: Karin, Thomas and Femke. Karin, you have been a very kind and motivating co-promotor, especially during the last stretch of my PhD. Because of many reasons, I think we can definitely call it a stretched last project that took longer than expected. Thank you for your patience at such times, but also for your quick replies in case I had questions and the many papers you send me in preparation for my defence. Thomas, thanks for your enthusiasm for my project and your helpful suggestions on the paper. Femke, thank you for helping me find my way through the clinical datasets. 
My collaborators at Actelion or later Janssen Pharmaceutical Companies: Suzanne Jansen and Rob Janssen. Suzanne: thank you for giving me so much background literature to start my project and for organizing the interview sessions. It gave me a very good impression of the impact of pulmonary hypertension and a network to start the research with. I enjoyed working with you very much. Rob, thank you for your enthusiasm for the project and our sessions on the progress of my research.

My former colleague dietitian Margje for motivating me to do the MSc Nutrition \& Health and start a career in science at all. You helped me convince myself that this was the right path for me. I always dreamed to contribute to improving nutritional interventions for patients and hopefully this thesis has done that a little bit.

My paranimfs and friends Marielle and Marije, thank you for being part of this $\mathrm{PhD}$ adventure, for the many times we talked during dinners, coffees, walks, by telephone or skype about the process, the difficulties, the frustrations, etc. Marielle, also thanks for staying at your house during times that I was in Wageningen and for taking care of me when I needed it. You truly honour your nickname within the PhD's at our department and I could not think of a better paranimf to have at my side! Marije, also thanks for listening to and understanding the struggles of being a working mom (especially in home-office during the Covid-19 pandemic) over the last almost 2 years. A problem shared is a problem halved.

My long-time friends Samantha and Stephanie: thank you for over 20 years of friendship! Although our lives took very different turns, I am happy that we always stay in contact. You have been interested in my project over the years and helped me to stay sane during difficult times.

Last but not least: Annette. Mam, dankjewel voor je luisterend oor en wijze raad tijdens dit hele $\mathrm{PhD}$ traject en eigenlijk ook tijdens alle andere momenten in mijn leven. Al ruim 35 jaar sta je altijd voor me klaar. Nu ik zelf moeder ben, weet ik pas echt wat dat betekent. 'Kan-niet ligt op't kerkhof en wil-niet ligt ernaast.' Met dat in het achterhoofd lukte het dit promotieboekje af te krijgen. 
Bennie: Pap, dankjewel voor je interesse in de artikelen die ik gepubliceerd heb (ook al kwamen die wellicht over als kippenduits) en voor de tientallen conceptversies tijdens het ontwerpen van de omslag van dit boekje. Ik ben heel erg blij met het resultaat!

Marcel, wat ben ik blij met zo'n grote broer als jij! We zijn op veel vlakken zo verschillend als twee kinderen van dezelfde ouders ook maar kunnen zijn. Desondanks kunnen we heerlijk over uiteenlopende dingen discussiëren. Dat heeft me over de jaren heen vaak geholpen mijn gedachten vorm te geven.

Birgit und Marit: Danke, dass ihr mich so herzlich in eure Familie aufgenommen habt! Ohne euch hätte ich mich nach den Umzug nach Leipzig nicht so zuhause gefühlt. Danke, dass ihr euch oft so lieb um Nora kümmert, sodass ich Zeit hatte (und habe) zu arbeiten und mich auch mal erholen kann.

Matthias, thank you for supporting this $\mathrm{PhD}$ adventure, for your help on uncountable many little parts of it and for motivating me during the many difficult times over the last years. Thank you for being such a great and responsible father to our daughter. The last 7 years have showed that we are a strong team. I hope that we can continue to build a meaningful, loving and sustainable family life together.

My lovely daughter Nora for bringing so much more joy and a different kind of purpose into my life, showing me where my priorities are and giving me a different view of the world. I hope that in the future, this thesis may be inspiration and proof that you can accomplish anything you want, even if things do not always work your way. 


\section{Curriculum vitae}

Paulina Susanna (Paulien) Vinke was born in Emmen, the Netherlands, on 25 October 1986. After completing secondary school at the Carmelcollege (KDC) in Emmen in 2005, she studied Nutrition \& Dietetics in Groningen. During her bachelor, she was active as a board member and chairman of a student party in the University Counsel and editor-in-chief of the student opinion magazine 'Nait Soez' $n^{\prime}$. She won the academy thesis prize for best bachelorthesis of the year. After completing her bachelor in 2010, she worked as a dietitian for 5 years. In 2011, she started the MSc Nutrition \& Health at Wageningen University. In 2013, she completed this with a masterthesis on mTOR regulation in liver and white adipose tissue in aging mice. In 2014, she started a job as a teaching assistant at the Nutrition \& Pharmacology group of Wageningen University. In 2015, she moved to Leipzig, Germany, and collaborated with dr. Klaske van Norren from Wageningen University and dr. Volker Adams of the Heart Center Leipzig to write grant proposals to start a $\mathrm{PhD}$ on nutritional interventions to prevent muscle wasting in pulmonary hypertension and heart failure. In 2016, she was rewarded the Deutscher Akademischer Austauschdienst (DAAD) DIA stipendium and started her PhD at Wageningen University, with guest employment at the Heart Center Leipzig to conduct an animal study on nutritional interventions to prevent muscle wasting in heart failure and pulmonary hypertension. In 2017, she was awarded a research grant of the dr. Catharine van Tussenbroek Fonds to continue this research. In 2018, she started a collaboration with Actelion Pharmaceuticals and wrote a literature review on nutritional status in PH patients. In 2019, she collaborated with dr. Karin Boomars of the Erasmus Medical Center Rotterdam to measure micronutrient status in PH patients. She supervised one MSc thesis student and four BSc thesis students and attended five scientific conferences. Paulien is an active member of the evidencebased food collective I am a Foodie since 2015, writing blogs and giving interviews in podcasts and the media on healthy nutrition and debugging food myths. 


\section{Selected publications}

Vinke, P., Koudstaal, T., Muskens, F., van den Bosch, A., Balvers, M., Poland, M., Witkamp, R.F., van Norren, K., \& Boomars, K.A. (2021). Prevalence of Micronutrient Deficiencies and Relationship with Clinical and Patient-Related Outcomes in Pulmonary Hypertension Types I and IV. Nutrients, 13(11):3923.

Vinke, P., Wesselink, E., van Orten-Luiten, W., \& van Norren, K. (2020). The use of proton pump inhibitors may increase symptoms of muscle function loss in patients with chronic illnesses. International Journal of Molecular Sciences, 21(1), 323.

Vinke, P., Bowen, T. S., Boekschoten, M. V., Witkamp, R. F., Adams, V., \& van Norren, K. (2019). Anti-inflammatory nutrition with high protein attenuates cardiac and skeletal muscle alterations in a pulmonary arterial hypertension model. Scientific Reports, 9, 10160.

Vinke, P., Jansen, S. M., Witkamp, R. F., \& van Norren, K. (2018). Increasing quality of life in pulmonary arterial hypertension: is there a role for nutrition? Heart Failure Reviews, 23(5), 711-722.

Bowen, T. S., Adams, V., Werner, S., Fischer, T., Vinke, P., Brogger, M. N., Mangner, N., Linke, A., Sehr, P., ... Labeit, S. (2017). Small-molecule inhibition of MuRF1 attenuates skeletal muscle atrophy and dysfunction in cardiac cachexia. Journal of Cachexia, Sarcopenia and Muscle, 8(6), 939-953. 


\section{Grant proposals}

2016: DAAD/OCW DIA Scholarship: Research grant to promote academic exchange between Dutch and German universities. Awarded: 3 Months.

2017: A2 Grant dr. Catharine van Tussenbroek Fonds for the execution of a study into the effect of nutritional and exercise interventions to prevent muscle wasting in a mouse model of pulmonary arterial hypertension. Awarded: 6 Months.

2017: Research Grant Actelion Pharmaceuticals for the publication of a literature review into the role of nutrition in pulmonary arterial hypertension. Awarded: 6 months.

2018: Research grant Actelion Pharmaceuticals for studying micronutrient deficiencies and relation with clinical- and patient-related outcomes in PAH and CTEPH patients. Awarded: 12 months.

Not awarded despite high scores:

2015/2016: DAAD Cotutelle research scholarship for binational PhD programme on nutrition and exercise intervention to prevent muscle dysfunction in heart failure. Review score 9/10.

2015/2016: DAAD short research scholarship for PhD research on nutrition and exercise intervention to prevent muscle dysfunction in heart failure. Review score $9 / 10$. 


\section{Overview of completed training activities}

Discipline specific activities

Courses

Training period in Leipzig Heart Center

Animal science art. 9 / FELASA Function B for

mice and rats

Conferences and meetings

$8^{\text {th }}$ Conference Cachexia, Sarcopenia \&

Muscle Wasting

$9^{\text {th }}$ Conference Cachexia, Sarcopenia \&

Muscle Wasting

$10^{\text {th }}$ Conference Cachexia, Sarcopenia \&

Muscle Wasting

11th Conference Cachexia, Sarcopenia \&

Muscle Wasting

12th Conference Cachexia, Sarcopenia \&

Muscle Wasting

\section{General Courses}

Intensive language course German B2

Healthy and sustainable diets: synergies and trade-offs

Career Orientation

\section{Other activities}

Preparation of research proposal

Meetings WUR

Meetings Herzzentrum

Coordinator Journal Club
Organiser and location

Year

Herzzentrum, Leipzig

2016

Radboud University,

Nijmegen

SCWD, Paris

2015

SCWD, Berlin

2016

SCWD, Rome

2017

SCWD, Maastricht

SCWD, Berlin

2019

InterDaF University of

2015

Leipzig

VLAG, Wageningen

WGS, online

2021

Herzzentrum, Leipzig 
The research described in this thesis was supported by the Deutscher Akademischer Austauschdienst (DAAD) in the form of a DIA stipendium, a research grant of the Dr. Catharine van Tussenbroek Fonds and research grants of Actelion Pharmaceuticals.

Financial support from Wageningen University for the publication of this thesis is gratefully acknowledged.

Thesis cover design: Bennie Vinke and Paulien Vinke

Lay-out: Matthias Schröter-Vinke, Bennie Vinke and Paulien Vinke

Printed by Gildeprint www.gildeprint.nl 



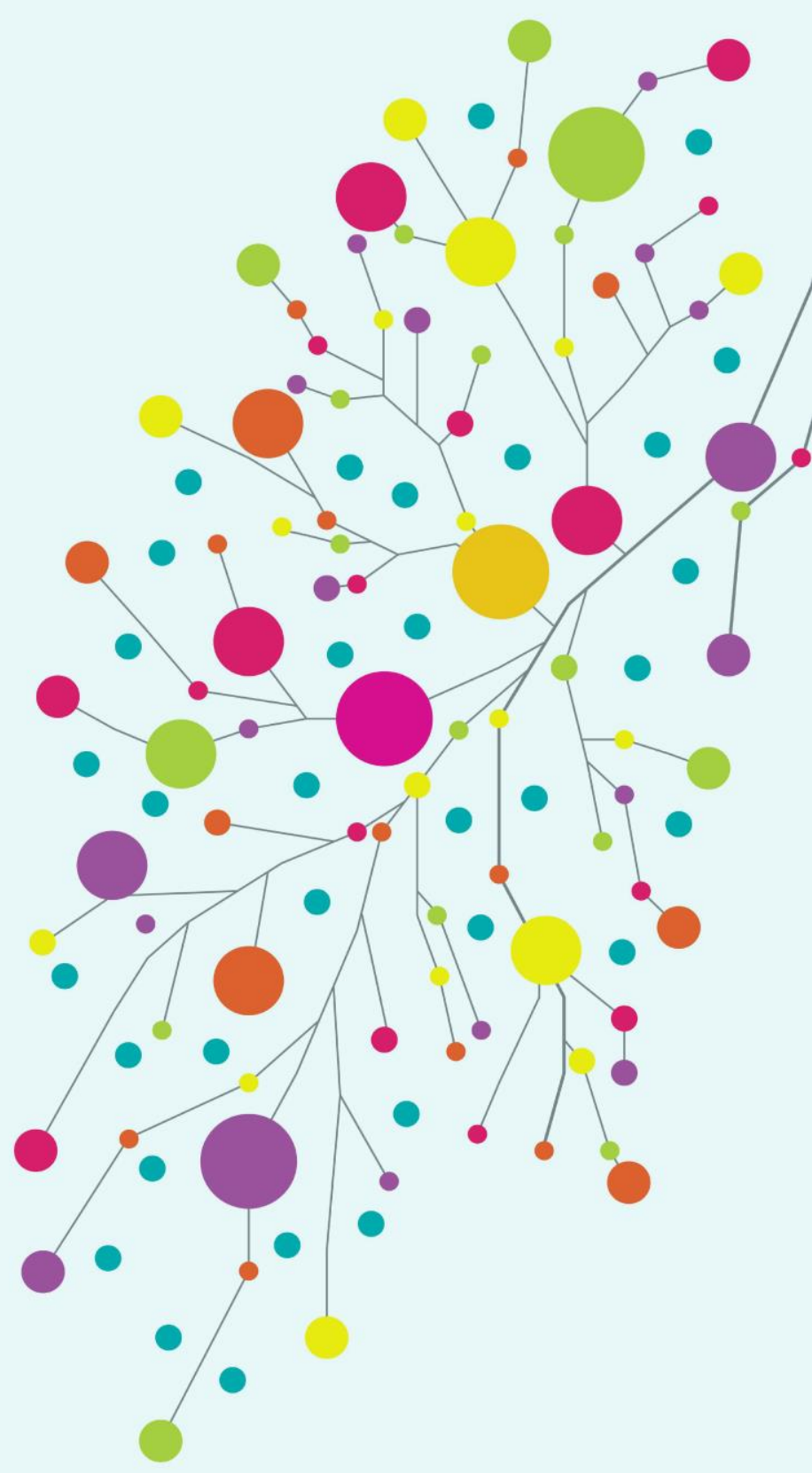

